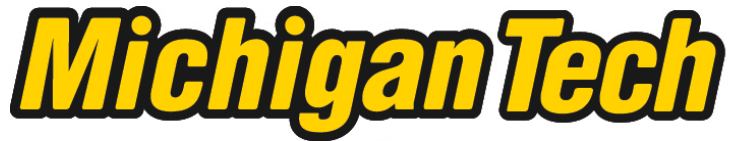 \\ Michigan Technological University Create the Future Digital Commons @ Michigan Tech
}

Dissertations, Master's Theses and Master's Reports - Open

Dissertations, Master's Theses and Master's

Reports

2005

\section{Model based experimental investigation on Powered Gait Orthosis (PGO)}

Huojin Cheng

Michigan Technological University

Follow this and additional works at: https://digitalcommons.mtu.edu/etds

Part of the Mechanical Engineering Commons

Copyright 2005 Huojin Cheng

\section{Recommended Citation}

Cheng, Huojin, "Model based experimental investigation on Powered Gait Orthosis (PGO)", Dissertation, Michigan Technological University, 2005.

https://doi.org/10.37099/mtu.dc.etds/354

Follow this and additional works at: https://digitalcommons.mtu.edu/etds

Part of the Mechanical Engineering Commons 


\title{
Model Based Experimental Investigation on Powered Gait
}

\section{Orthosis (PGO)}

BY

Huojin Cheng

\begin{abstract}
A DISSERTATION
Submitted in partial fulfillment of the requirements

for the degree of

DOCTOR OF PHILOSOPHY

(Mechanical Engineering-Engineering Mechanics)

MICHIGAN TECHNOLOGICAL UNIVERSITY

2005
\end{abstract}

Copyright $(\odot$ Huojin Cheng 2005 
This dissertation, "Model Based Experimental Investigation on Powered Gait Orthosis (PGO), " is hereby approved in partial fulfillment of the requirements for the degree of DOCTOR OF PHILOSOPHY in the field of Mechanical Engineering-Engineering Mechanics.

DEPARTMENT or PROGRAM:

Mechanical Engineering-Engineering Mechanics

Signatures:

Dissertation Advisor

Dr. John Beard

Department Chair

Dr. William W. Predebon

Date 


\title{
MODEL BASED EXPERIMENTAL INVESTIGATION ON POWERED GAIT ORTHOSIS (PGO)
}

\author{
Huojin Cheng \\ Department of Mechanical Engineering - Engineering Mechanics \\ Michigan Technological University, 2005
}

\begin{abstract}
Research on rehabilitation showed that appropriate and repetitive mechanical movements can help spinal cord injured individuals to restore their functional standing and walking. The objective of this paper was to achieve appropriate and repetitive joint movements and approximately normal gait through the PGO by replicating normal walking, and to minimize the energy consumption for both patients and the device. A model based experimental investigative approach is presented in this dissertation.

First, a human model was created in Ideas and human walking was simulated in Adams. The main feature of this model was the foot ground contact model, which had distributed contact points along the foot and varied viscoelasticity. The model was validated by comparison of simulated results of normal walking and measured ones from the literature. It was used to simulate current PGO walking to investigate the real causes of poor function of the current PGO, even though it had joint movements close to normal walking. The direct cause was one leg moving at a time, which resulted in short step length and no clearance after toe off. It can not be solved by simply adding power on both hip joints.

In order to find a better answer, a PGO mechanism model was used to investigate different walking mechanisms by locking or releasing some joints. A trade-off between energy consumption, control complexity and standing position was found.
\end{abstract}


Finally a foot release PGO virtual model was created and simulated and only foot release mechanism was developed into a prototype. Both the release mechanism and the design of foot release were validated through the experiment by adding the foot release on the current PGO. This demonstrated an advancement in improving functional aspects of the current PGO even without a whole physical model of foot release PGO for comparison. 
With God all things are possible.

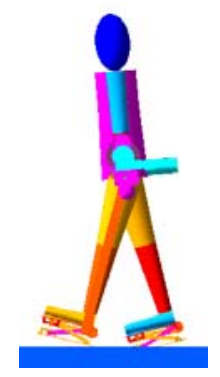




\section{ACKNOWLEWGDGEMENTS}

I am glad that I can still manage to get my job done, but I hate to leave this place with so many loved people. My thesis is full of my tears and people's love. First, I would like to thank my advisor, Dr. John Beard for his support financially, guidance, understanding and love. Then I would like to express my appreciation to Dr. William Predebon, Dr. Oner Arici and Dr. Gordon Parker who extended a Ph.D. position to my wife when I was in hardest time. Thanks also to other committee members Dr. Tammy Donahue and Dr. Allan Struthers for their time and inputs to improve my work. Also I would like to thank Jesse Nordeng for making the prototype, Dr. Jason Blough for his help in data testing and Suzanna Post for her review.

A special thanks goes to Mike Ditto's family who took care of my kids for almost two months while Laurie Ditto gave up her part time job, pastor Paul Marin of Hope Fellowship Who helped us financially and spiritually, and all other people from Hope Fellowship for their love, prayers and fasting.

I can never say thanks enough to my wife Zhiru Shi. She has to take care of the whole family and her studies, and never gives up.

Finally I would like to extend my thanks to the people of whole Copper County for their love, care, prayers and donations. I know that no matter how agreeable to your ear my words are, they are still pale to your love. My family will remember your love forever and ever. 


\section{TABLE OF CONTENTS}

\section{ABSTRACT i}

ACKNOWLEWGDGEMENTS iv

TABLE OF CONTENTS v

LIST OF FIGURES xi

LIST OF TABLES xvi

1 INTRODUCTION 1

1.1 Motivation 1

1.2 Overview on Rehabilitation Devices 2

1.3 Overview on Simulation of Human Walking 14

1.4 Model Based Experimental Investigative Approach 19

1.5 Thesis Outline 20

2 HUMAN WALKING 22

2.1 Normal Gait 22

2.2 Kinematics 24

2.3 Kinetics 25

2.4 Energy Expenditure 26

2.5 Ground Reaction Forces 28

2.5.1 Vertical Reaction Force 28

2.5.2 Anterior-posterior (AP) GRF 30

2.6 Summary 31 


\section{SIMULATION OF HUMAN WALKING 33}

\subsection{Human Model 33}

3.2 DOFs and Constraints 35

3.3 Joint Motion Functions 36

3.4 Foot-Ground Contact Model 37

3.4.1 Linear Viscoelastic Model 38

3.4.2 Pseudo-Coulomb friction Model 38

\subsubsection{Contact points 39}

3.5 Upper Body Balance Control 40

3.6 Analysis of Simulation Results 42

3.6.1 Ground Reaction Force 43

3.6.2 Sliding and Penetration 46

3.6.3 The Center of Mass 49

3.6.4 Joint Motions 50

3.6.5 Joint Moment and Power Consumption 51

3.6.6 Control Torque 55

3.7 Summary 56

\section{SIMULATION OF CURRENT PGO PHYSICAL MODEL 57}

4.1 Current PGO Model 57

4.2 Human in Current PGO Model 60

\subsection{Joint Motion Function 61}

4.4 Analysis of Simulation Results 62

4.4.1 Ground Reaction Force 63 
4.4.2 Sliding and Penetration 64

4.4.3 The Center of Mass 66

4.4.4 Joint Motions 67

4.4.5 Joint Torque and Power Consumption 68

4.4.6 Control Torque 70

4.5 Discussion 71

4.6 Summary 77

5 PGO MECHANISM INVESTIGATION 78

5.1 PGO Mechanism Model 78

5.2 Human in PGO Mechanism Model 80

5.3 Joint Motion Function 80

5.4 Simulation 81

5.5 Analysis of simulation results 83

5.5.1 Ground reaction force 83

5.5.2 Sliding and Penetration 85

5.5.3 The Center of Mass 87

5.5.4 Joint Motions 88

5.5.5 Joint Moment and Power Consumption 89

5.5.6 Control Torque 93

5.6 Discussion 95

5.7 Comparison of Foot Release PGO with RGO 98

5.8 Investigation on the Adjustment of Step Length of Foot Release PGO 100

5.9 Summary and Conclusion 102 
5.9.1 Summary 102

5.9.2 Conclusion 102

\section{DESIGN OF THE FOOT-RELEASE PGO 103}

6.1 Design of Hip joint 103

6.1.1 Cam Follower 103

6.1.2 Cam Profile 104

6.1.3 Driving Power for Cam 106

6.2 Design of Foot Release 107

6.2.1 Release Mechanism 107

6.2.2 Worm Gear 109

6.2.3 Driving Power for Foot Release 111

6.3 Foot Release PGO Virtual Model 112

6.4 Simulation of Foot-release PGO Virtual Model 114

6.5 Analysis of Simulation Results 115

6.5.1 Joint Motions 115

6.5.2 Ground Reaction Force 116

6.5.3 Sliding and Penetration 117

6.5.4 The Center of Mass 118

6.5.5 Moment and Power Consumption 119

6.5.6 Contact Force between Cam and Follower 120

6.5.7 Moment on Gear 120

6.5.8 Power Ratio 121

6.5.9 Control Torque 122 
6.6 Physical Realization of Foot-release Mechanism 123

6.7 Step motor and Control 123

6.8 Summary 125

7 VALIDATION OF THE MECHANISM AND DESIGN OF FOOT-RELEASE 126

7.1 Simulation of Current PGO with Foot-release 126

7.1.1 Simulation 126

7.1.2 Ground Reaction Forces 127

7.1.3 Moment and Power Consumption 129

7.1.4 Power Ratio 130

7.1.5 Control Torques 131

7.2 Testing System Set up 132

7.3 Measured Results 133

7.3.1 Ground Contact Force With No Extension of Swing Leg 133

7.3.2 Ground Contact Force With Five Degree Extension 134

7.4 Discussion 135

7.5 Summary 136

8 CONCLUSIONS AND RECOMMENDATIONS 137

8.1 Summary and Conclusions 137

8.2 Contributions 138

8.3 Recommendations for Future Work 139

REFERENCE 141 


\section{APPENDIX A 150}

Ideas Models 150

Adams Models 150

\section{APPENDIX B 151}

Matlab Files 151

B.1 regression.m 151

B. 2 bezier.m 161

B.3 bezier_regression.m 175

B.4 hipcam.m 182

B.5 Data_processing.m 186 


\section{LIST OF FIGURES}

HGO, RGO (Jefferson 1990) 4

Powered Hip Orthosis (PHO) (Downes 1994) 6

WBC Orthosis 7

Lokomat 9

AutoAmbulator (healthsouth.com) 10

Mechanized Gait Trainer (MGT) 11

Bio-Responsive Motion System (BRMS) 12

HAL 13

Time dimensions of the gait cycle (Inman, 1981) 23

Kinematics (from MAC system by Andreas Kranzl, Vienna) 25

Joint moments and power (Normalized data is from Winter's book) 26

Vertical ground reaction force (GRF) during walking (Soutas-Little, http://www.vard.org/ mono/gait/soutas.htm) 28

Vertical displacement and acceleration of the center of gravity (c.g.) of the body during walking 29

Anterior-posterior (AP) ground reaction force (GRF) 30

Ground reaction forces (Normalized data is from Winter's book) 31

Human model 35

Fourier series and measured data of three joint angles 37

Contact points 40

Primitive joints 40 
Constrained human model 42

Simulation of human walking 43

Ground reaction force 44

Sliding of normal walking 46

Friction comparison 47

Step length of normal walking 48

Penetration of normal walking 49

Displacement of COM 50

Angles of three joints 51

Torque and power for hip 53

Torque and power for knee 53

Torque and power for ankle 54

Power for normal walking 54

Balance control moment 55

PGO model 57

Joint angles of PGO 59

Comparison of hip and knee angles of PGO with those of human walking 59

Human in PGO 61

Fourier series of motor rotation speed of PGO 62

Simulation of PGO walking 63

Vertical ground reaction force in PGO walking 64

Progressional force in PGO walking 64

Sliding of PGO walking 66 
Penetration of PGO walking 66

Displacement of COM in PGO walking 67

Hip and knee angles in PGO walking 68

Torque and power of hip joint in PGO walking 69

Torque and power of knee joint in PGO walking 69

Total power of hip and knee in PGO walking 70

Control torque in PGO walking 71

Relation of hip and stride in normal walking 73

Relation of hip and step in PGO walking 73

Step length of PGO walking 74

Friction 75

Stance phase 76

PGO mechanism model 79

Human in PGO mechanism model 80

Fourier series of foot release mechanism 81

Simulation of foot knee release PGO walking 82

Simulation of foot release PGO walking 82

Ground reaction force on PGO mechanism model 84

Impact force comparison 84

Friction comparison 85

Sliding of PGO mechanism model 86

Penetration of PGO mechanism model 87

COM of PGO mechanism model 88 
Joint angles of PGO mechanism model 88

Moment and power for foot knee release PGO 91

Moment and power for knee release PGO 91

Moment and power for foot release PGO 92

Power comparison of PGO mechanism model 92

Control torque of PGO mechanism model 94

Comparison of control torque of PGO mechanism model 94

Step length of PGO mechanism model 96

Comparison of stride lengths or distances 96

PGO mechanism model hip and knee angles 98

Hip mechanism 100

Adjustment of hip angles in foot release PGO 101

Adjustment of step length in foot release PGO 101

Contact force of cam and follower 104

Vector loop 104

Cam profile 105

Hip virtual model 106

Cylinder release mechanism 108

Worm gear release mechanism 108

Torque on gear in worm gear release model 109

Torque from single worm gear release simulation 110

Driving torque and power on worm 111

Foot release virtual model 112 
Foot release PGO virtual model 113

Simulation of Foot release PGO virtual model 114

Joint motions in virtual model 116

Cylinder release displacement 116

Ground reaction force in virtual model 117

Sliding and penetration of virtual model 118

COM of virtual model and cylinder release 118

Moment and power in virtual model 119

Contact force of cam and follower in virtual model 120

Running torque on gear in virtual model 121

Step length of virtual model 121

Control torque in virtual model 122

Prototype of foot release 123

Simulation of current PGO with foot release movement 127

Simulation of current PGO without release movement 127

Comparison of ground reaction forces 128

Comparison of moment and power consumption 129

Comparison of walking distance with or without release 130

Comparison of control torque with or without release 131

Testing system of ground reaction force for trailing leg 133

Measured ground reaction force with no extension 134

Measured ground reaction force with 5o extension 135 


\section{LIST OF TABLES}

Dimension of Human Model 33

Calculated and Measured Power and Moment in Normal Walking 52

Control Torque of Normal Walking 55

Torque and Power of Current PGO 68

Control Torque 71

Maximum force and Stance phase of PGO Mechanism Walking 83

Sliding of PGO mechanism Walking 85

Torque and Power for Mechanism Model 90

Control Torque of PGO Mechanism Model 93

Comparison of Power Ratio 95

Comparison of Cylinder and Worm Gear Release 115

Maximum force and Stance phase of Virtual Model 117

Moment and Power for Virtual Model 119

Power Ratio of Cylinder Release and Virtual Model 122

Control Torque of Virtual Model 122

Comparison of Ground Reaction Forces 128

Moment and Power for Current PGO with Foot Release 129

Power Ratio of Current PGO with or Without Release Movement 130

Control Torque of Current PGO With or Without Release Movement 131 


\section{INTRODUCTION}

\subsection{Motivation}

How to restore the functional standing and ambulation to spinal cord injured individuals has been a major task for many researchers in rehabilitation. The most progress has been made in rehabilitation theory. Recent research (Harkema 2001) has suggested that the spinal cord network has the intrinsic capability to interpret complex sensory information, such as what position the legs are in and how much load is being borne by the legs. With that information, the spinal cord can generate activation patterns for standing, stepping and other actions. And because the neural pathways are very plastic, patients are able to teach the spinal cord to learn the motor task through the appropriate and repetitive mechanical movements. Several mechanical devices have been developed to accomplish this task. The recent developed Lokomat (Colombo 2000; 2001) and AutoAmbulator(healthsouth.com) are using computer controlled normal gait patterns to drive patients' legs. These machines can provide patients with accurate mechanical movements; however, they are very expensive and patients can not move around. The construction for a lightweight, simple, inexpensive Powered Gait Orthosis (PGO) (Ruthenberg 1994; 1997) was developed even earlier than these machines, however, it is still in the experiment stage. A prototype was developed a few years ago. From the experiment, it was observed that joint movements of hip and knee close to normal walking did not bring in close normal gait. It proposed some new problems like one leg moving at a time, short step or stride, body twisting forward, big ground friction or no clearance and so on. Even if the leg sequence 
is solved, the toe-off friction problem is still there. Thus, the purpose or the main task of this project is to improve the function of the current PGO.

First, a better understanding of normal walking, PGO walking and the difference between them is needed; the true causes of the poor function of the current PGO needs to be found. Then the design can be modified to replicating the normal gait more accurately. Model simulation is convenient and insightful tool for investigating biomechanics or mechanics of human walking and PGO walking. Therefore in this paper a model based experimental investigative approach is presented to simulate the normal walking and PGO walking to visualize the different walking mechanism, predict the function and optimize the structure of the modified PGO, and validate the design through the experiment. On the other hand, with an ideal simulation, design questions could be answered using the computer model, reducing dependency on costly prototypes and avoiding unnecessary loss from some misunderstanding or mistake.

The final goals of this paper are to achieve appropriate and repetitive joint movements and approximate normal gait through the PGO, and to minimize the energy consumption for both patients and the device. Before the approach to achieve these goals is discussed, a look back at what have been done in the area of rehabilitation devices and simulation of human walking is presented.

\subsection{Overview on Rehabilitation Devices}

Many devices have been employed in the effort to restore functional standing and ambulation to spinal cord injured individuals. They can be classified into three categories: mechanical, Functional Electrical Stimulation (FES) and hybrid of these two. Many-chan- 
nel FES-only systems are currently impractical clinical alternatives because of the strength limitations and rapid fatigue associated with FES-activated muscle as well as the complexity of required FES controllers (Tashman 1995). Hybrid systems produce jerky gait because of manual stimulation, although these systems utilize FES-activated lowerextremity musculature to provide propulsive force while taking advantage of the weightbearing support and stability offered by orthosis. Mechanical devices seem to provide more normal gait and are controlled by computer. Passive orthosis have several differentiating features. The main characteristic of the Hip Guidance Orthosis (HGO) shown in Figure $(1-1)$ or the "Parawalker", is that its hip joints are free to flex and extend between stops. The Reciprocating Gait Orthosis (RGO) (Figure (1 - 1)) differs in that a cable or linkage system coupling two hip joints ensures extension on one side, causing flexion on the other side (Douglas 1983; Jefferson 1990; Whittle 1991). The main feature of Moorong Medial Linkage Orthosis (Moorong MLO) is a low friction linkage to prevent abduction of the swing-through leg while the body is tilted to create ground clearance for the swing leg. At the same time, this prevents abduction of the stance leg under body weight (Middleton 1998). 


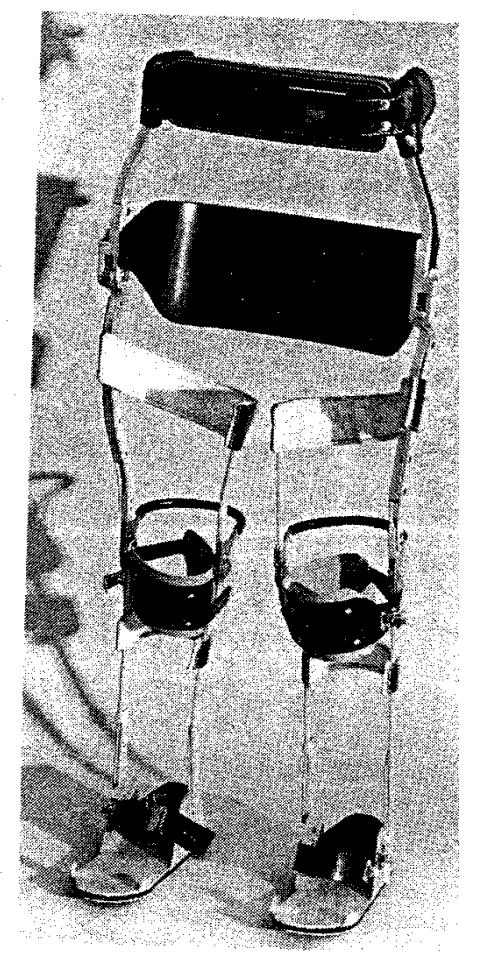

(a)

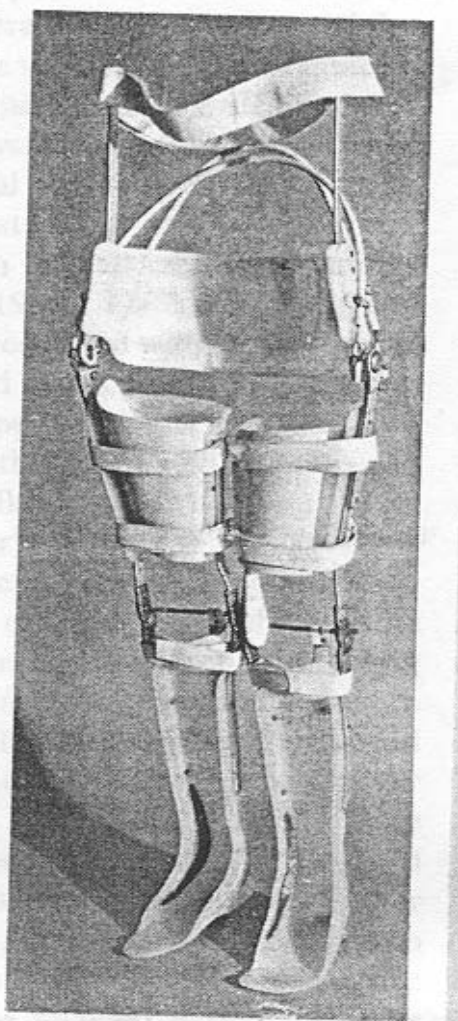

Figure (1 - 1) HGO, RGO (Jefferson 1990)

Arguably, the most effective of currently available passive devices is the Reciprocating Gait Orthosis (RGO) (Jefferson 1990; Whittle 1991). Walking in RGO is achieved by pulling the trunk forward, using crutches or rollator, then tipping the pelvis so that the trailing leg is lifted clear of the ground, thus allowing it to move forward and take a step. However, for individuals to ambulate with the RGO, even for short distances at low speeds with the assistance of crutches or rollator, the high energy or upper body strength demanded restricts their widespread use. Compared to a normal individual, the typical RGO user walks approximately one third as fast and consumes four times as much energy per meter travel (Tashman 1995). It is also pointed out that an RGO user walking at $1 \mathrm{~ms}^{-1}$ would have to do 14 times more work than a normal person (Bernardi 1995). The causes of the 
high energy consumption can be shown through gait analysis. In the case of paraplegic walking, the needed movement of the hip joint and pelvis is much greater than that of a healthy subject. This is of course a necessary consequence of the patient having to lift and rotate the pelvis to swing a leg through its swing phase due to the necessary locking of the knee joint, while Bowden cables on the orthosis connecting the hip joints transfer the reverse motion to the stance leg, propelling the subject forward. This action is, of course, extremely tiring (Downes 1994). In fact, the tilt of pelvis and the jerky gait make the center of gravity swing widely and the acceleration of the center of gravity not as smooth as normal people. These also cause more energy consumption.

To solve these problems, many external powered devices have been developed. The Powered Hip Orthosis (PHO) shown in Figure $(1-2)$ is a modified RGO by applying external power to the hip joints while disconnecting the transfer cable but leaving the knees still locked (Downes 1994). Motors are controlled by microprocessor. Walking in this device, the user still has problem of foot/ground clearance. 


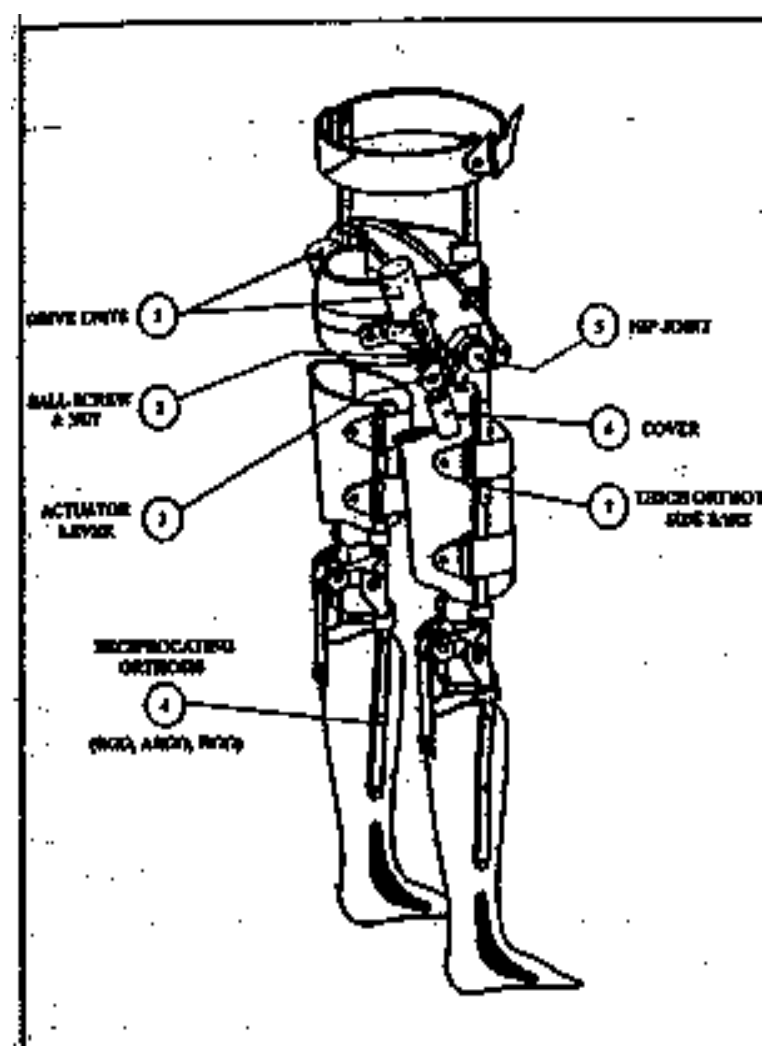

Figure (1 - 2) Powered Hip Orthosis (PHO) (Downes 1994)

The weight bearing control (WBC) orthosis shown in Figure (1 - 3) is also a modified RGO with two variable sole-plates for foot/ground clearance during walking (Yano 1997). The variable sole-plates are powered by a $\mathrm{CO}_{2}$ liquid air gas tank. During the swing phase, the sole-plate retracts to provide foot/ground clearance; before the swing foot touches the ground, the sole-plate extents to the original thickness. However, the hip joints are still manual. 


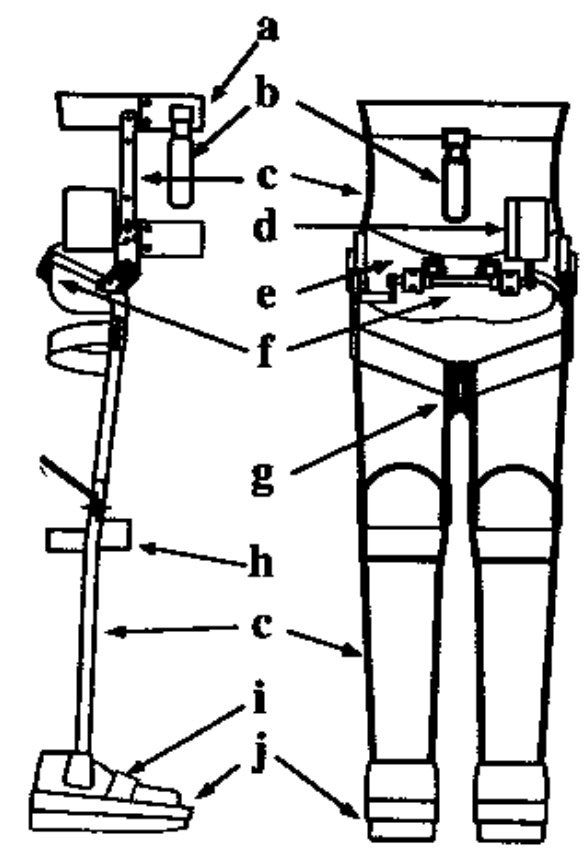

Figure (1 - 3) WBC Orthosis

The Powered Gait Orthosis (PGO) is developed by Dr. Beard and his team (Ruthenberg 1994; 1997). The current PGO prototype is one degree of freedom for each leg. The hip joint is powered by a DC motor through a four-bar mechanism; the knee joint is actuated with the use of a cam-modulated linkage, the cam profile being machined into the face of the lower gear (driven by the motor). Since the cam follower is captured in a slotted cam profile, it always remains in contact with the cam during flexion or extension. Hopefully the motor can drive hip joint to move swinging leg forward and at the same time flex the knee joint to make clearance for the swinging leg. In fact, because two joints are coupled and only one leg moves at a time, it is only an approximation of real biped 
walking, still having some problems that can be seen from the simulation results of the current PGO.

Recently, more new devices have been developed. Lokomat(Figure (1 - 4)) (Colombo $2000 ; 2001)$ is a treadmill training device with a driven gait orthosis (DGO). The orthosis has four revolute joints that accommodate hip and knee flexion/extension for each leg. The joints are driven by precision ball screws connected to DC motors. Parameters such as the hip width, thigh length and shank length can be manually adjusted to fit individual patients. The weight of the orthosis is supported by a parallelogram mechanism that moves in the vertical direction and is counterbalanced by a gas spring. A harness system can partially unload the patient's weight and control the upper body's balance. The hip and knee motors are controlled by computer using gait pattern which is generated by tracking the four reference angle trajectories recorded in another experiment, where a healthy subject was walking in the orthosis. 

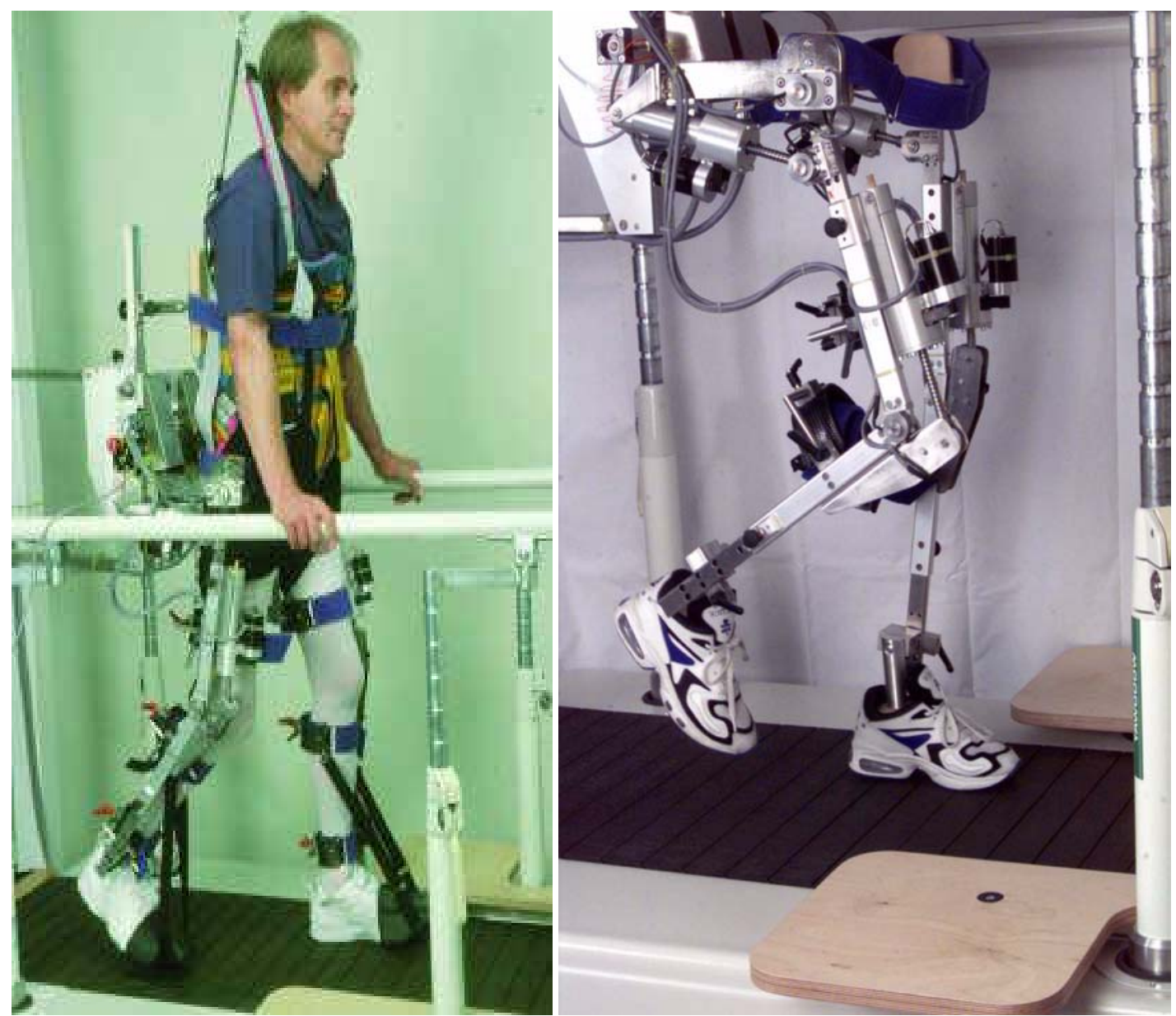

Figure (1 - 4) Lokomat

The AutoAmbulator (Figure (1 - 5)) (healthsouth.com) is also a sophisticated treadmill device that is unparalleled in its ability to help patients accurately replicate normal walking patterns. While no detailed report on it is available, the sophisticated features include:

- An overhead harness system to fully support the patient

- Mechanically powered brace to move the patient's legs

- Numerous computerized sensors to track vital signs, movement and contact speed

- Automatic speed adjustments based on contact speed

- An emergency button that allows the patient or therapist to stop the machine if needed 

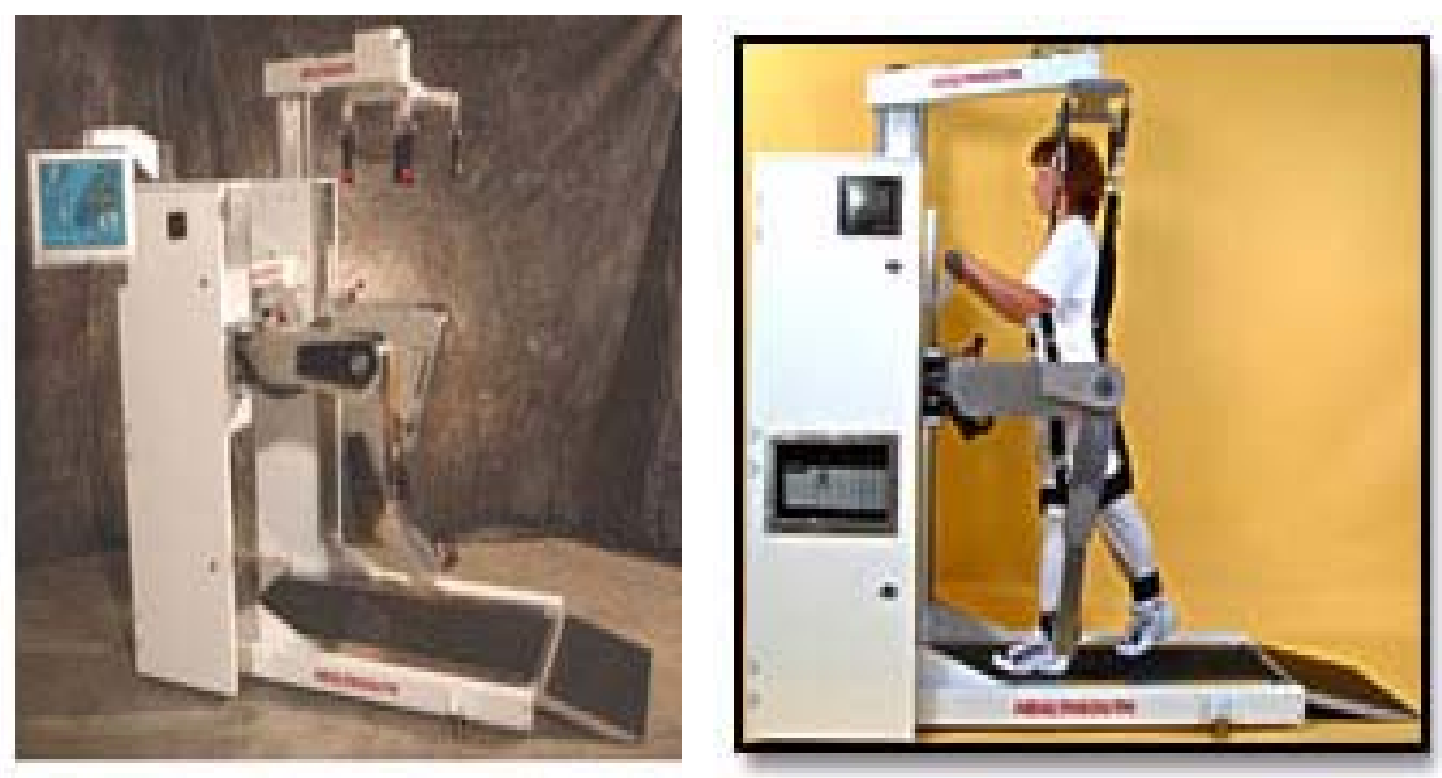

Figure (1 - 5) AutoAmbulator (healthsouth.com)

The Mechanized Gait Trainer (MGT) (Figure (1 - 6)) (Hesse 2000) is a different device. The gait trainer is based on a doubled crank and rocker gear system. It consists of two footplates positioned on two bars (couplers), two rockers, and two cranks that provides the propulsion. The low backward movement of the footplates simulates the stance phase while the forward movement simulates the swing phase. The system generates a different movement at the tip and at the rear of the footplate during the swing. The tip of the plate follows an arc-like movement corresponding to the length of the rocker. The rear end is lifted during swing so that the footplate itself is inclined during swing. Furthermore, the crank propulsion is modified by a planetary gear system to provide a ratio of 60 percent to 40 percent between stance and swing phases. However, there is no direct control for knee movement. 




Figure (1 - 6) Mechanized Gait Trainer (MGT)

Another similar device called Robotic Walking Simulator (Schmidt 2002a; 2002b) has been developed by the same group. It consists of two robots. In each robot there are two independently driven electrical linear motors moving on a common rail, which are used for the two linear base joints to achieve the dynamic performance. The linkage principle is similar to a crank and slider mechanism. At the crank pivot, a short robot arm is attached. The footplate is mounted the distal end of the robot arm. The horizontal and vertical position of the footplate depends on the position of both linear motors, whereas the angular position is determined by a rotatory motor located inside the robot arm. To some degree, this device can achieve more accurate trajectories of feet and stair climbing training. 
Bio-Responsive Motion System (BRMS) (Figure (1 - 7)) (Hirata 2002) is a novel exerciser for two hip, knee, and ankle joints of spastic patients. It is composed of a link mechanism, bed for the patient to lie on, an operating/monitoring panel, a patient's monitoring panel, and control unit. One pair of two mechanical arms of the BRMS move the targeted lower extremity. The link mechanism comprises one pair of actuated arm mechanisms and splints. One of the mechanism supports the thigh of the patient, giving two-degree-of-freedom rotating/linear motion. The another drives the lower leg, giving three-degree-of-freedom rotating/linear motion. The other drives the ankle joint. Hip, knee, and ankle have a wide range of motion with the BRMS.

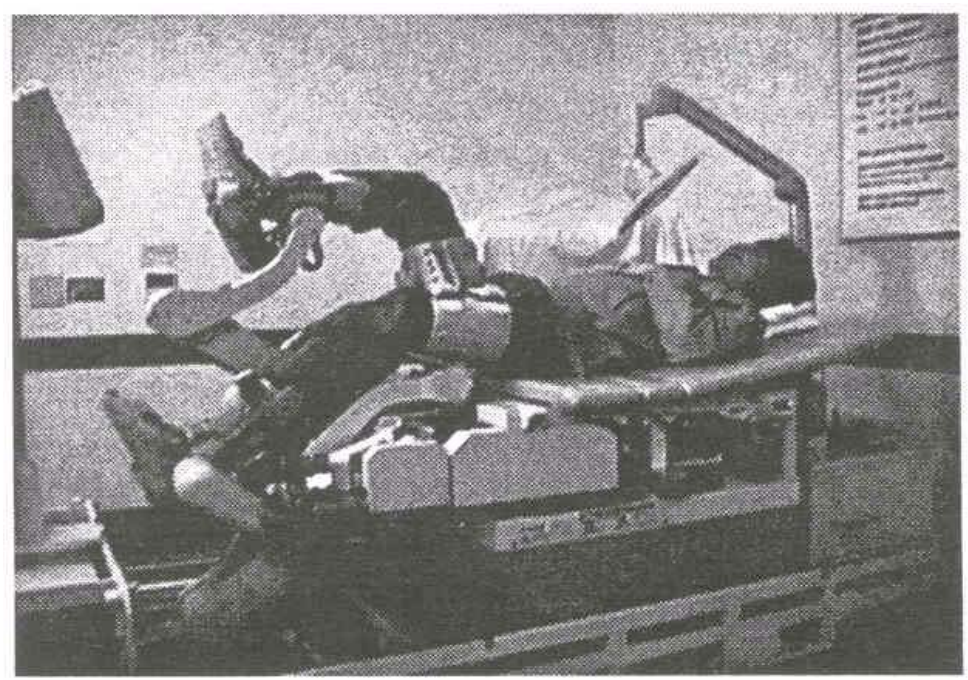

Figure (1 - 7) Bio-Responsive Motion System (BRMS)

The Hybrid Assistive Leg (HAL) (Figure (1 - 8)) (Kawamoto 2002; Kasaoka 2001; Lee 2002a; Lee 2002b) is an EMG-based power assistant system for walking aid. It is composed of three main parts: skeleton and actuator, controller, and sensor. The skeletal system of HAL consists of an exoskeletal frame with six joints (hip, knee and ankle joints for each leg; each joint is 1 DOF) and four actuators driving hip and knee joints. The actu- 
ator has a DC servo motor with the harmonic drive gear. The control system consists of compact PC, driver circuits, power supply, and measuring module which are packed in the back pack. The sensor systems are used to detect HAL and operator's condition and estimate the assist force. The rotary encorder is prepared to measure the each joint angle, force sensors are installed in the front and rear sole of the foot to measure the floor reaction force (FRF) sensor, and the myoeletricity sensors are attached on the surface of the skin of leg to estimate torques for knee and hip joints.

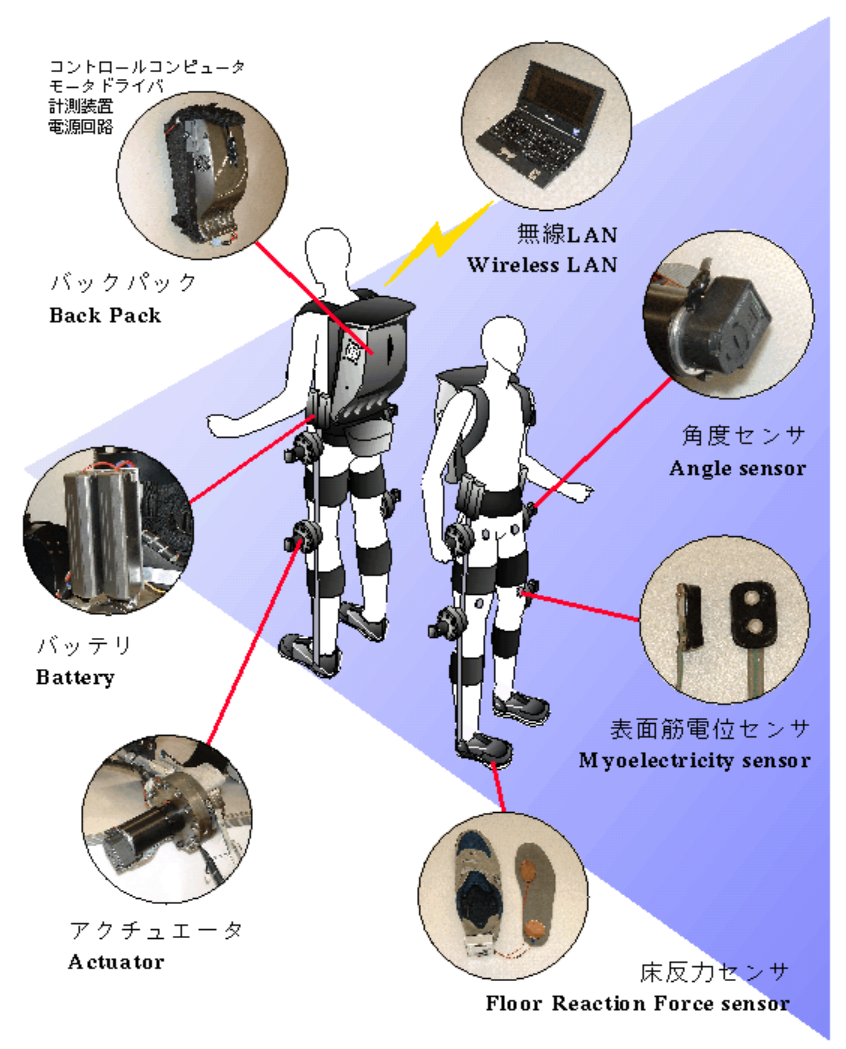

Figure (1 - 8) HAL 


\subsection{Overview on Simulation of Human Walking}

Simulation of human walking is relevant to three fields: computer graphics, robotics and biomechanics. Researchers in computer graphics have explored the animation technique, including: keyframing, kinematic, dynamic, hybrid of kinematic and dynamic, and motion editing. Keyframing technique provides motion by specifying the joint angles over time. The joint angle is interpolated from the initial configuration to the final one, with several intermediate configurations if necessary. It is hard to find the interpolation functions in human walking that require a large amount of non-linear coordination among the body parts.

Bruderlin and Calvert (Bruderlin 1989;1988) built a keyframeless animation of walking system. Dynamic simulation provides the low-level control detail necessary to define a motion; kinematic algorithms are applied to calculate all the body angles from the motion of the dynamic model. The system can generate a wide range of walking pattern by changing the three primary parameters.

Ko (Ko 1994) also used kinematic and dynamic techniques in animation. Kinematic technique was used to initiate and generate goal oriented intentional motion, and dynamic technique was applied at each frame to adjust the kinematic motion to achieve dynamic soundness without affecting the goal achievement.

The foot ground contact is rarely considered. Vasilonikolidakis and Clapworthy performed inverse Lagrangian dynamics on articulated models, focusing on the ground reaction force during the locomotion case (supposed to be the only external force).

Researchers in robotics have explored control techniques for legged robots that walk, run, balance and perform other activities. McGeer (McGeer 1990; 1993) was the first to 
study passive dynamic walking. It was shown that the passive walkers can walk smoothly and stably down a shallow incline without any sensing, control, or actuation, and the walking cycle was smooth, efficient and natural.That means the gait can be sustained simply by interaction of gravity and inertia in a limited cycle.

Honda P2 (Hirai 1998) and P3 each has 12 actuated degrees of freedom in the lower body -- three in each hip, one in each knee, and two in each ankle. The robot is primarily controlled by playing back pre-recorded joint trajectories acquired from direct measurements of human subjects. Three additional controllers modify the trajectory in order to maintain balance in light of disturbances, terrain, or modelling errors. A ground reaction force controller modifies joint angle trajectories to achieve the desired zero moment point (ZMP) and thus conform to uneven terrain. A model ZMP controller shifts the desired ZMP by changing the ideal body trajectory when the robot is about to tip over. A foot landing position controller changes the stride length to compensate for changes in the body trajectory made by the model ZMP controller. With this control scheme, the robot can walk fairly fast, walk up and down stairs, and turn in place. An intelligent walking technology featured with a predicted movement control is added to a new generation ASIMO (asimo.honda.com). ASIMO can walk more smoothly and more naturally.

Researchers in biomechanics have been exploring the mechanism of human walking physiologically and physically. In their reviews (Zajac 2002; 2003), Zajac et. al provided insight into muscle coordination of human walking from simple models to complicated neuro-musculo-skeletal models. In our review, however, the focus is on approaches for modelling and simulation of human walking to solve problems in this area. Chow et. al (Chow 1971) used a model of two-link lower extremity (a ball foot was fastened to the 
shank) combined with optimization theory to simulate normal gait. The performance criterion is to minimize the sum total of mechanical energy expenditure by the muscle-activating system which is proportional to the integral of the square of the net moment. Onyshko and Winter (Onyshko 1980) modelled the human body with three segments. The three segments are two lower limbs, and the head, arms and trunk (HAT) as one segment. Heel, toe or ankle was pivoted on the floor for four different walking phases. In their later studies (Gilchrist 1996; 1997), they presented a nine-segment three-dimensional model, including a two-part foot. Ju et al (Ju 1988) only simulated the double support phase of human gait. Amirouche et al (Amirouche 1990) modelled the human body using five segments with the ankle of the stance leg pivoted on the floor. Koopman (Koopman 1995) used an eightsegment model combined with optimization to reconstruct and predict the bipedal walking. Pandy et al (Pandy 1988a;1988b; 1989a; 1989b) simulated single stance phase of human walking to quantify the influence of individual gait determinants on the ground reaction forces generated during normal, level walking. In their recent research (Anderson 1999; 2001a; 2001b; 2002;Pandy 2001), they presented a more complicated musculo-skeletal model combined with dynamic optimization to predict muscle excitations and thus reproducing the features of normal gait or jumping. Neptune et al (Neptune 2001) used a musculo-skeletal model to study the contributions of individual muscles to trunk support and progression. Wright et al (Wright 1998; 2000) also used a musculo-skeletal model to study the joint and muscle loading and investigate the injury mechanism of ankle sprains. Taga (Taga 1998; 2000) used a neuro-musculo-skeletal model to investigate the dynamic interaction between the neural system, the musculo-skeletal system and the environment in the maintanence of stable gait. Hase (Hase 2002a; 2002b; 2002c; 2002d) used the same 
method to study human locomotion, but his model is with 14 rigid links and 60 muscles, and the neural system was presented by the rhythm generator system, the sensory feedback system and the peripheral system. Some others were interested in pathologic gait. Tashman (Tashman 1995) simulated swing phase of paraplegic ambulation in a RGO to study the dynamics of RGO-assisted gait and to explore FNS control strategies for the design of hybrid RGO/FNS systems. Englbrecht (Englbrecht 2001) simulated crutch walking with measured foot ground reaction force and crutch ground reaction force to determine the moments and power on elbow and shoulder joints.

Our purpose to simulate human walking is to understand the kinematics and dynamics of human walking and validate some models which will be used for the simulation and the design of the PGO, through comparing the simulation results with measured data. Thus we are more interested in the segment model and the forward \& inverse dynamic approach. Because the foot ground contact model is important for this research.

Gilchrist and Winter (Gilchrist 1996; 1997) used a two-part, viscoelastic foot model. A total of nine contact elements, arranged down the midline of the foot, were used; the vertical ground reaction forces resulted from linear spring/damper systems in each of the contact elements. The damping coefficient was modelled non-linearly as a function of the amount of spring compression to prevent large step increases in force as each damper was brought into play. The shear forces were modelled as linear functions of the velocity of the contact points.

Van Den Bogert (Van Den Bogert 1989) used foot ground contact model for simulation of quadrupedal locomotion. The GRF were approximated vertically by a linear viscoelastic model and horizontally by a pseudo-Coulomb friction model. 
Wojtyra (Wojtyra 2000) also used a linear viscoelastic model for vertical force, the damping $\mathrm{c}$ is nonlinear function of penetration. In fact, in ADAMS a step function is used to prevent discontinuity at the contact starting point (zero penetration). A pseudo-Coulomb friction model was used for horizontal force. To prevent singularity, a small constant was added to the denominator.

Gerritsen (Gerritsen 1995) used a nonlinear model for vertical force in simulation of impact in heel-toe running and a Coulomb friction model for horizontal force. Ouezdou and Bruneau (Ouezdou 1998; Bruneau 1999) used a distributed nonlinear model for vertical force and a linear model for horizontal force. Silva (Silva 2001) modelled the contact through a linear spring-damper in the horizontal direction and a linear spring with a nonlinear damper in the vertical direction.

Wright and Neptune (Wright 1998; Neptune 2000) modelled the contact through 66 discrete independent contact elements. A nonlinear spring and a nonlinear damper were used for vertical force, and a pseudo-Coulomb friction model was used for horizontal direction.

Anderson (Anderson 1999) modelled foot in two parts. Five spring-damper units are distributed over the sole of each foot. The vertical force is exponential to the height of the foot above the ground; the horizontal force is linear.

Most of them did not show the values of the penetration and sliding through which the accuracy of modeling can be shown. Also, contact models were used to simulate different conditions including walking, running and jumping. However, we can start with a simple one, then refine the model to minimize penetration and sliding. 


\subsection{Model Based Experimental Investigative Approach}

It is told in section 1.1 why we wanted to use the model based experimental investigation approach. It can visualize the walking mechanism, provide us insight into biomechanics or mechanics of human walking and PGO walking, help us to better understand normal walking and PGO walking, predict the function and optimize the structure of the modified PGO. The model based experimental investigative approach presented in this paper is the investigation based on dynamic simulation of human walking model and human in PGO walking mode, and validation through experiment. The details are:

- Through dynamic simulation of human walking, to validate the foot ground contact model and the balance control strategy of upper body by comparing the calculated foot ground contact force and the movement of COM with measurements; To obtain forces and energy consumptions for joint's movements while human was treated as a non-living physical body like a robot;

- Applying the same foot ground contact model and upper body balance control strategy to the current PGO physical model to show its performance and find the difference by comparing the results of normal walking with those of current PGO walking;

- Applying the same foot ground contact model, upper body balance control strategy and some joint's movements to different PGO mechanism model to explore the final scheme of the modified PGO;

- To size and design the modified PGO virtual model and simulate the virtual model to refine the design and predict its function;

- To validate the virtual model through the experiment. 


\subsection{Thesis Outline}

Human walking is overviewed in Chapter 2. It provides us a direct and simple look into the normal gait, kinematics and dynamics of human walking based on the measurement, which will be used in the next chapters to provide joint motions as driving power in the simulation and also to provide a basis or standard to validate the simulation. It includes normal gait, energy consumption, kinematics and kinetics of human walking.

Simulation of human walking is presented in Chapter 3. It shows us how to make a model in I_DEAS then simulate it in ADAMS. An average Hanavan male model is used as human model. The data of joint motions of lower extremities is from Winter's book (Winter 1991). Foot ground contact model is the nucleus of this part and is also the basis for the simulation of other models. By comparison of simulated results and the measured data from Chapter 2, this model is validated. It includes human model, joint motion functions, foot-ground contact model, upper body balance control and simulation results analysis.

Current PGO physical model is simulated in Chapter 4. The same foot ground contact model and upper body control strategy as those validated in Chapter 3 are used in the simulation. It provides insight into the current PGO walking. By comparing the simulation results with those of normal walking, the true causes of poor function of the current PGO are explored. It includes current PGO model, human in current PGO model, joint motion function, simulation results analysis and problem discussion.

Simulation of PGO mechanism models is described in Chapter 5 Three PGO mechanism models are compared to find a trade-off between energy expenditure, control simplicity and functions. 
Chapter 6 presents the design of the foot-release PGO, a trade-off found in Chapter 5. The design is detailed, including solid models and drafts. Only the foot-release mechanism is physically realized. The driving power for the foot-release mechanism is a step motor which can be started by a push button and stopped at the exact position by inner control program.

Both mechanism and design of foot-release are validated in Chapter 7. The ground reaction forces in both conditions, walking in current PGO with foot release and without it, are measured and compared to show the effectiveness of the mechanism and design of foot release. The simulation of current PGO with foot-release further proves the effectiveness of the mechanism of foot release.

A final conclusion is summed up in Chapter 8 . 


\section{HUMAN WALKING}

Before modeling started, a thorough understanding of the mechanism, kinematics and dynamics of human walking which is based on the measurement and observation is needed. They are the basis to the modeling and simulation. Therefore, some important conception and data on human walking are summed up in this overview.

\subsection{Normal Gait}

The gait cycle is a time interval or sequence of motion occurring from heelstrike to heelstrike of the same foot. The gait cycle has been broadly divided into two phases: stance phase and swing phase. These phases can then be further subdivided and discussed in terms of percentage of each within the gait cycle. This is diagrammatically represented in Figure (2 - 1) by Verne T. Inman (Inman 1981). 

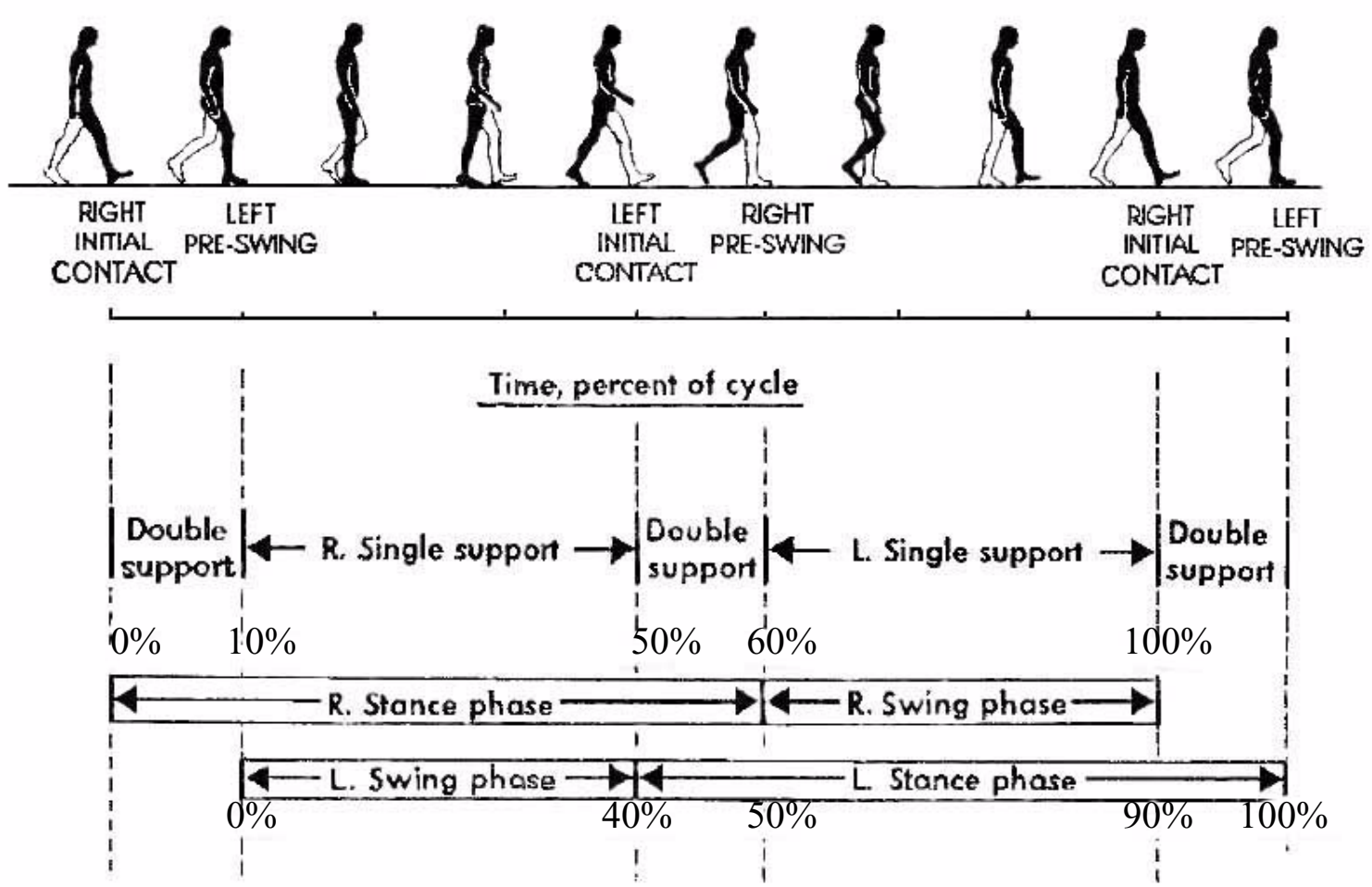

Figure (2 - 1) Time dimensions of the gait cycle (Inman, 1981)

The stance phase is about 60 percent of the gait cycle and can be subdivided into double-leg and single-leg stance. In double-leg stance, both feet are in contact with the ground. At an average walking speed, it represents 10 percent of the entire gait cycle, but decreases with increased walking speed and ultimately disappears as one begins to run. At slower walking velocities the double-leg support times are greater. Single-leg stance comprises up to 40 percent of the normal gait cycle.

The swing phase is described when the limb is not weight bearing and represents 40 percent of a single gait cycle. It is subdivided into three phases: initial swing (acceleration), midswing, and terminal swing (deceleration). Acceleration occurs as the foot is lifted from the floor and, during this time, the swing leg is rapidly accelerated forward by hip and knee flexion along with ankle dorsiflexion. Midswing occurs when the accelerat- 
ing limb is aligned with the stance limb. Terminal swing then occurs as the decelerating leg prepares for contact with the floor.

\subsection{Kinematics}

The joint kinematics describes the relative position and orientation of one body segment to the adjoining one. The relative orientation of one body segment to another defines the joint angles. Four joints angles are shown in Figure (2 - 2) (CGA Normative database, http://guardian.curtin.edu.au/cga/). Large movements happen in sagittal plane. That is the partial reason for why the model walking only in sagittal plane is simulated. Because PGO restricts the motion in this plane, the simulation in this plane make it comparable to PGO walking. The joint angles in sagittal plane are used as driving power in the simulation. The curves are shown in Figure (2 - 3). The data is from Winter (Winter 1991). 



Figure (2 - 2) Kinematics (from MAC system by Andreas Kranzl, Vienna

\subsection{Kinetics}

Kinetics mainly deals with the individual muscle forces, the moment generated by those muscles across a joint, the mechanical power patterns (rate of generation or absorption by muscles, or rate of transfer between segments), or energy patterns (segment or 
total body). The data of joint moments and power from Winter's book is normalized. It is converted to that of an average adult male $(73.5 \mathrm{~kg})$ by timing $73.5 \mathrm{~kg}$. The curves are shown in Figure (2 - 3).



Figure (2 - 3) Joint moments and power (Normalized data is from Winter's book)

\subsection{Energy Expenditure}

The ultimate source of energy for bodily work is the oxidation of foodstuffs. Consequently, the measurement of the oxygen consumption of the body provides a measure of the energy expenditure of the body.

During gait, three main events occur in which energy is consumed. This includes controlling forward movement during deceleration toward the end of swing phase, shock 
absorption at heelstrike, and in propulsion during push off (when the center of gravity is propelled up and forward).

In natural walking, from Inman (Inman 1981), energy expenditure is

$$
E_{w}=\frac{E_{0}}{\left(1-V / V_{u}\right)^{2}}(\mathrm{cal} / \mathrm{min} / \mathrm{kg}),
$$

Where average $E_{0}=28, V$ is the speed and $V_{u}$ is the limit of $V, V_{u}=240 \mathrm{~m} / \mathrm{min}$.

From Perry's book, the energy expenditure for an adult at the Customary Walking Speed (CWS) is $12.1 \mathrm{ml} / \mathrm{kg} / \mathrm{min}$. Converted to calorie, $\mathrm{E}=12.1 * 5=60.5 \mathrm{cal} / \mathrm{min} / \mathrm{kg}$. The CWS for male adult is $82 \mathrm{~m} / \mathrm{min}$. In terms of Eqn $(2-1), \mathrm{E}_{\mathrm{W}}=64.61 \mathrm{cal} / \mathrm{min} / \mathrm{kg}$.

For an average male adult $(73.5 \mathrm{~kg})$ the energy expenditure is $73.5 * 60.5 * 4.1868(\mathrm{~J}) / 60$ $=310$ Watts. It is estimated that roughly one quarter of that is mechanical power (Rose 1994). It is 77.5 Watts. This data will be used to validate the model.

A human's center of mass (COM) is located just anterior to the second sacral vertebra, midway between both hip joints. The COM deviates from the straight line in vertical and lateral sinusoidal displacements. The net effect is a smooth, sinusoidal translation of the COM through space along a path that requires the least amount of energy. The least amount of energy is required when a body moves along a straight line, with the COM deviating neither up nor down, nor side to side, but that is not the case for walking.

With respect to vertical displacement: the COM goes through rhythmic upward and downward motion as it moves forward. The highest point occurs at midstance; the lowest point occurs at time of double support. The average amount of vertical displacement in the adult male is approximately $5 \mathrm{~cm}$.

With respect to lateral displacements: as weight is transferred from one leg to the other, there is shift of the pelvis to the weight-bearing side. The oscillation of the COM 
amounts to a side-to-side displacement of approximately $5 \mathrm{~cm}$. The lateral limits are reached at midstance.

\subsection{Ground Reaction Forces}

\subsubsection{Vertical Reaction Force}

Let us consider each component of the GRF separately. The largest is the vertical component and accounts for the acceleration of the body's center of mass in the vertical direction during walking. A typical plot of the vertical ground-reaction force is shown in Figure $(2-4)$, where the vertical reaction force is expressed as percent of body weight $(\% \mathrm{BW})$.

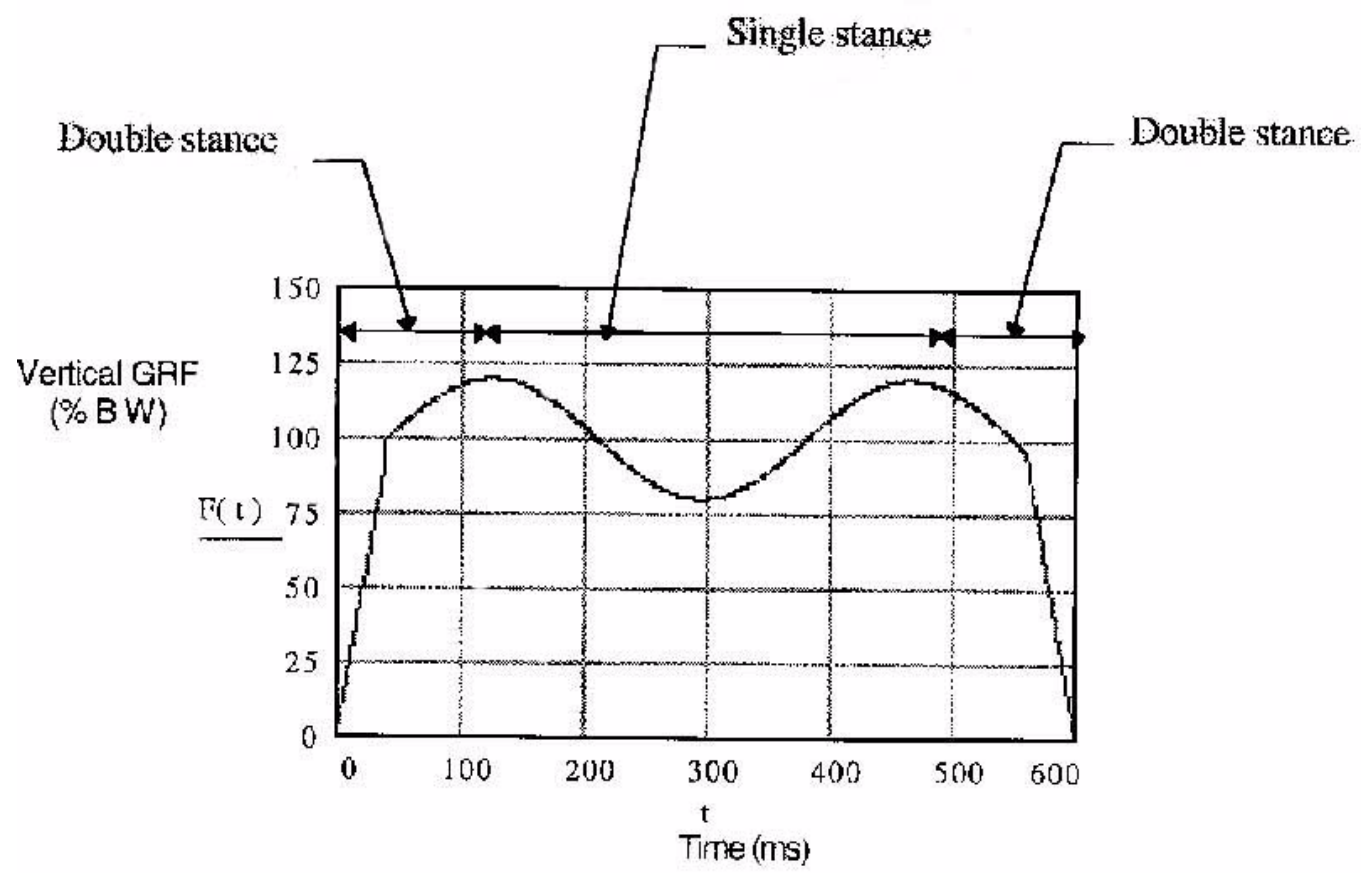

Figure (2 - 4) Vertical ground reaction force (GRF) during walking (SoutasLittle, http://www.vard.org/mono/gait/soutas.htm)

This curve is sometimes called the $\mathrm{M}$ curve because it resembles that letter. During the first $100 \mathrm{~ms}$, the GRF goes to a maximum of $120 \% \mathrm{BW}$ during the double stance 
phase. During single stance phase, the vertical GRF drops to about $80 \% \mathrm{BW}$ and for the more dynamic walker the vertical GRF drops to 60 to $70 \%$ BW. At first, it seems unusual that the GRF should be less than body weight during single stance when only one foot is on the ground. This is made clearer if the vertical position of the center of mass of the body during the gait cycle is examined. The center of mass is located around the center of the pelvis, ignoring changes due to arm position, and executes a sinusoidal motion rising and falling about $10 \mathrm{~cm}$ in space during walking, as shown in Figure (2 - 5).

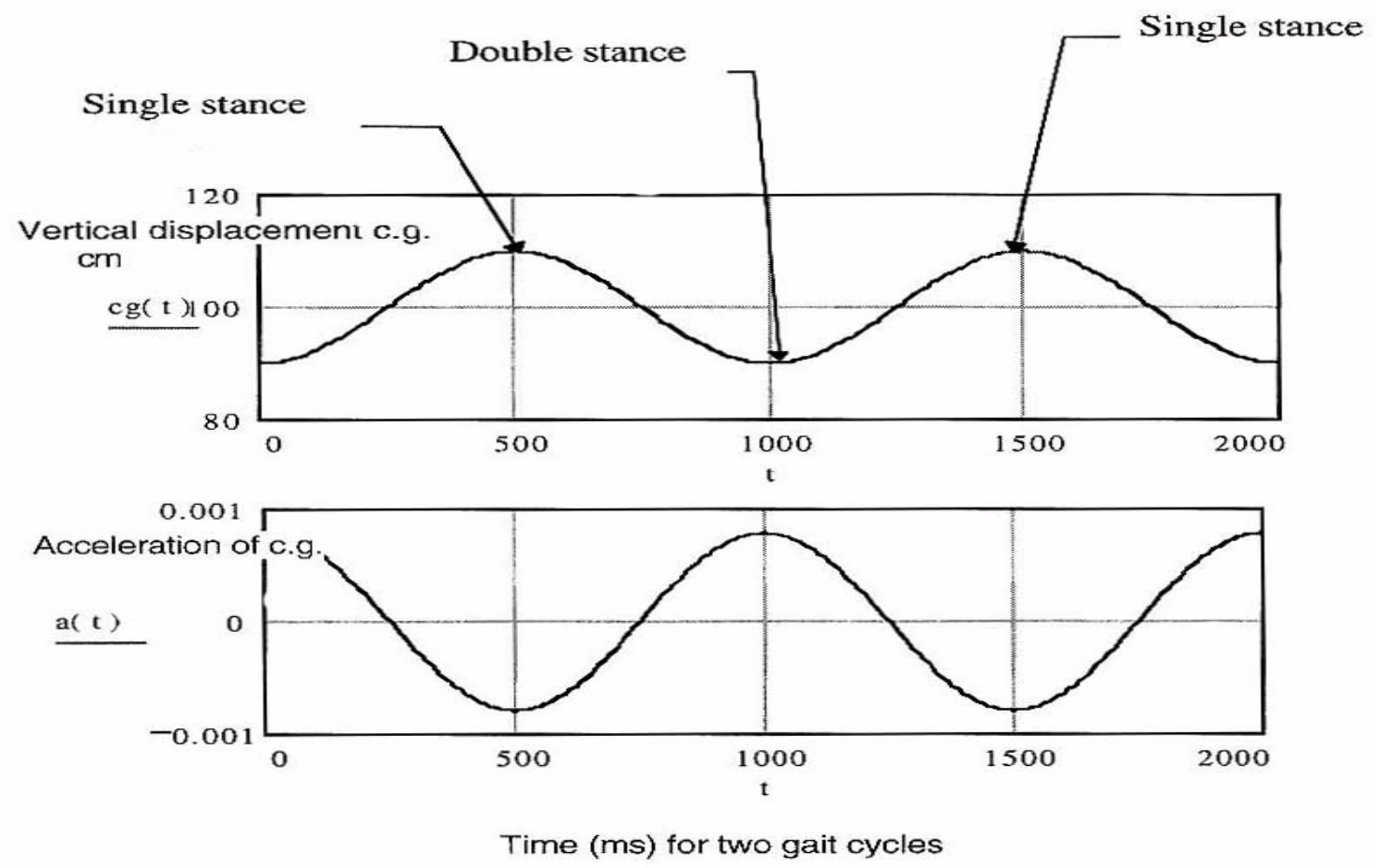

Figure (2 - 5) Vertical displacement and acceleration of the center of gravity (c.g.) of the body during walking

The acceleration of the center of mass in the vertical direction is shown below the displacement of the center of mass. It can be seen that this is opposite in sign at each point in 
the gait cycle. If the entire body is treated as a mass on a spring, the magnitude of the GRF can be more easily understood.

\subsubsection{Anterior-posterior (AP) GRF}

The anterior-posterior (AP) GRF is first a braking force to mid-stance, followed by propulsion. It usually represents a sine curve with an amplitude of $25 \% \mathrm{BW}$, as shown in Figure (2 - 6). The AP GRF is braking for approximately 50 percent of stance phase followed by propulsion. The area under any segment of this curve represents the impulse or the time integral of the force.

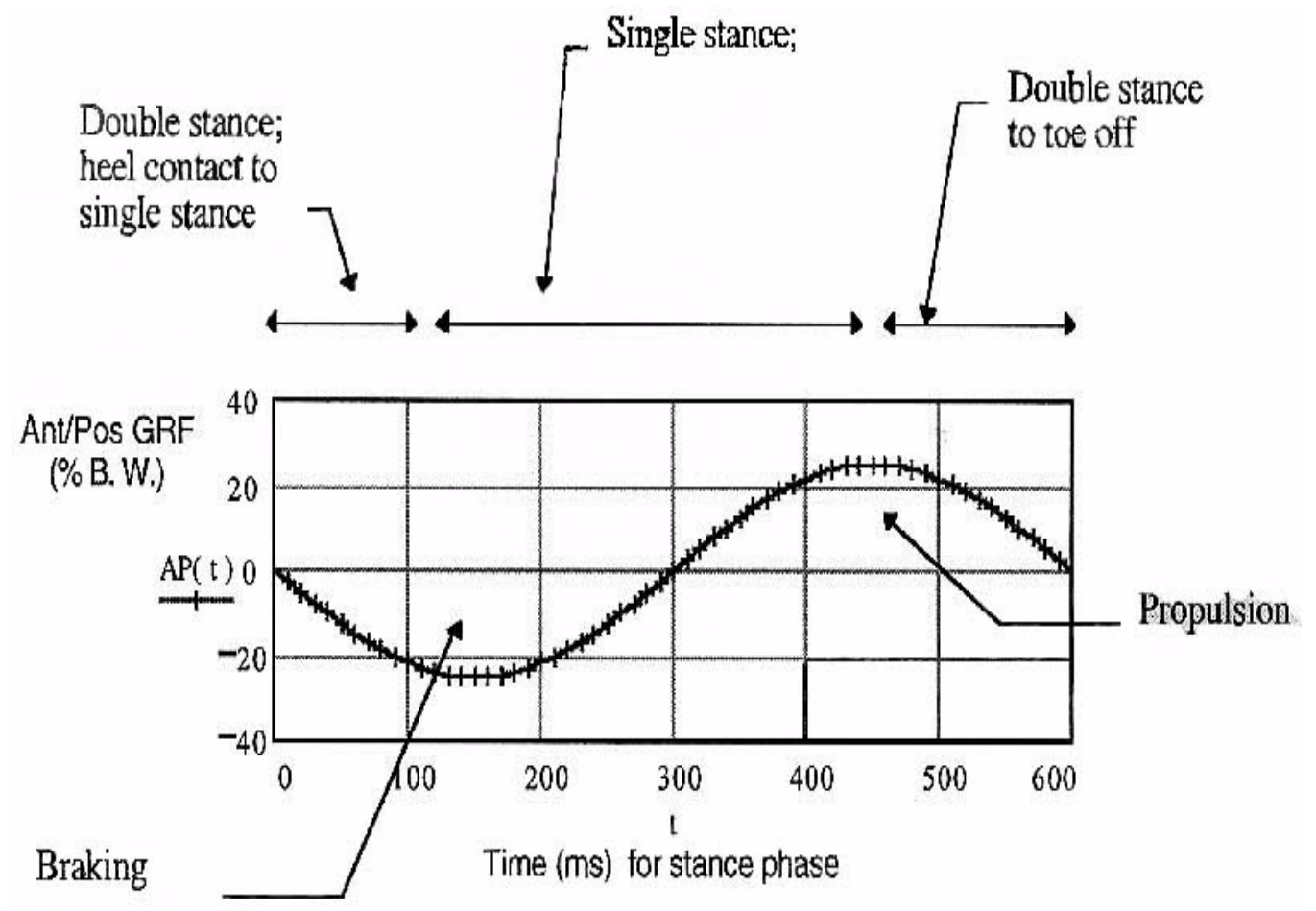

Figure (2 - 6) Anterior-posterior (AP) ground reaction force (GRF)

The braking impulse should be approximately equal to the propulsion impulse for balanced gait left to right. The total impulse in the AP direction for a full gait cycle should be zero, as the impulse is equal to the change in momentum in the forward direction. 
The data from Winter's book is normalized by the weight. For an average male adult (73.5 kg used in simulation), both vertical and horizontal reaction forces are shown in Figure $(2-7)$.
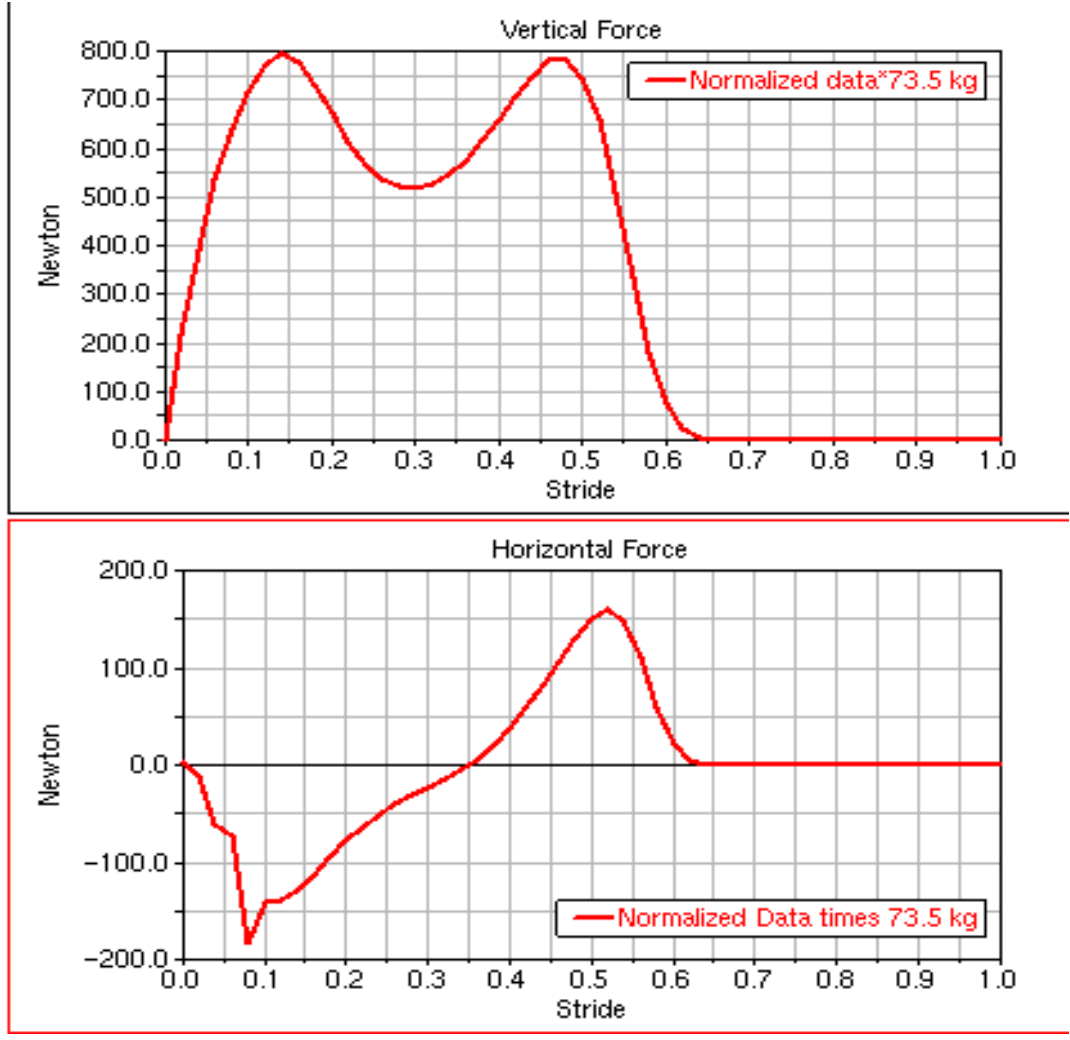

Figure (2 - 7) Ground reaction forces (Normalized data is from Winter's book)

\subsection{Summary}

This chapter gave us a simple look into the data prepared for simulation. The stance phase is $60 \%$ of gait cycle, COM is up and down $5 \mathrm{~cm}$, mechanical power is 77.5 Watts for an average adult male $(73.5 \mathrm{~kg})$. The data of hip, knee and ankle angles from Winter's book will be used as driving power in the simulation. Other data like joint moments, 
power and ground reaction forces are prepared from normalized data by multiplying 73.5 to validate the simulation. 


\section{SIMULATION OF HUMAN WALKING}

\subsection{Human Model}

A personalized mathematical model of the human body for evaluating inertial properties based on anthropological data and body mass distribution has been developed by Hanavan (Hanavan 1964). The human model adopted in this project was based on this model. The model can be used to evaluate the inertial parameters of the human body in any desired body configuration. These parameters are (Seireg 1989):

1. mass of body segments

2. centers of mass of body segments

3. mass moments of inertia of body segments

4. center of gravity of the whole body

5. principal axes of inertia of the whole body

The dimension of the model used here is a 50th percentile male (weight $=161.9 \mathrm{lb}$. or $73.5 \mathrm{~kg}$, height $=69.1 \mathrm{in}$. or $1.755 \mathrm{~m})$ and some properties are shown in Table $3-1$ on page 33 .

Table (3 - 1): Dimension of Human Model

\begin{tabular}{|c|c|c|c|c|c|}
\hline Segment & $\begin{array}{c}\text { Half length } \\
\text { of minor } \\
\text { axis }\end{array}$ & $\begin{array}{c}\text { Half length } \\
\text { of major } \\
\text { axis }\end{array}$ & $\begin{array}{c}\text { Segment } \\
\text { length }\end{array}$ & $\begin{array}{c}\text { Segment } \\
\text { weight }\end{array}$ & $\begin{array}{c}\text { Segment } \\
\text { density }\end{array}$ \\
\hline (inches) & $\begin{array}{c}\text { RR } \\
\text { (inches) }\end{array}$ & $\begin{array}{c}\text { SL } \\
\text { (inches) }\end{array}$ & $\begin{array}{c}\text { SW } \\
(\mathrm{lbs})\end{array}$ & $\begin{array}{c}\text { D } \\
\left(\mathrm{lb} / \text { in. }^{3}\right)\end{array}$ \\
\hline \hline Head & 6.25 & 3.58 & 12.5 & 12.8 & 0.040 \\
\hline
\end{tabular}


Table (3 - 1): Dimension of Human Model

\begin{tabular}{|c|c|c|c|c|c|}
\hline & $\begin{array}{l}\text { Half length } \\
\text { of minor } \\
\text { axis }\end{array}$ & $\begin{array}{l}\text { Half length } \\
\text { of major } \\
\text { axis }\end{array}$ & $\begin{array}{l}\text { Segment } \\
\text { length }\end{array}$ & $\begin{array}{c}\text { Segment } \\
\text { weight }\end{array}$ & $\begin{array}{c}\text { Segment } \\
\text { density }\end{array}$ \\
\hline Segment & $\begin{array}{c}\mathrm{R} \\
\text { (inches) }\end{array}$ & $\begin{array}{c}\mathrm{RR} \\
\text { (inches) }\end{array}$ & $\begin{array}{c}\mathrm{SL} \\
\text { (inches) }\end{array}$ & $\begin{array}{l}\text { SW } \\
\text { (lbs) }\end{array}$ & $\begin{array}{c}D \\
\left(1 \mathrm{lb} / \text { in. }^{3}\right)\end{array}$ \\
\hline Upper torso & 6 & 4.25 & 7.9 & 22.5 & 0.036 \\
\hline Lower torso & 6.6 & 4.18 & 15.6 & 52.2 & 0.039 \\
\hline Hand & 1.85 & 1.85 & 3.7 & 1.16 & 0.043 \\
\hline Upper arm & 1.98 & 1.74 & 13.5 & 5.0 & 0.034 \\
\hline Forearm & 1.74 & 1.08 & 10.9 & 3.0 & 0.043 \\
\hline Upper leg & 3.57 & 2.29 & 15.1 & 16.4 & 0.040 \\
\hline Lower leg & 2.29 & 1.42 & 15.1 & 7.9 & 0.048 \\
\hline Foot & 1.45 & 0.94 & 10.5 & 2.37 & 0.050 \\
\hline
\end{tabular}

The model shown in Figure (3 - 1) consists of 14 rigid segments shown in Table 3 - 1 on page 33 to ensure correct position of center of gravity and inertial properties of each segment. It is built in the IDEAS, then imported into ADAMS. It also has 3 fixed joints and 10 revolute joints. Revolute joints constrain the model to move only in the sagittal plane. The reasons to make this simplification are:

1. Almost all mechanical devices to restore ambulation are designed this way to control the balance of the upper body

2. Flexion and extension represent the major movements at most joints. Therefore they contribute significantly to performance in most tasks.

3. Simulation of normal walking in the sagittal plane make it comparable with device assisted walking, especially PGO. 



Figure (3 - 1) Human model

\subsection{DOFs and Constraints}

A totally unconstrained rigid part has 6 DOFs, fixed joint has 6 constraints, and revolute joint has 5 constraints. The model has 14 rigid parts, 3 fixed joints and 10 revolute joints. Then DOF of the model is $16(6 * 14-3 * 6-10 * 5=16)$. Therefore, enough forces or motions or joints must be assigned to constrain the model. Ten joint motion functions will constrain 10 DOFs, leaving 6 DOFs. Three ground reaction forces control 3 translate DOFs. In order to control 3 rotational DOFs, more joints or torques are needed. Therefore 
in Balance control two parallel primitive joints are used together to control 3 rotational DOFs, even though one parallel joint has two rotational constraints. Then the model will be fully constrained.

\subsection{Joint Motion Functions}

All moving joints are powered by motion functions. The data for lower extremities is obtained from measurement. The data for shoulder and arm are obtained through Bezier curve fitting by estimating some important positions of these joints. All the data from measurement were discrete. However, for the simulation purpose, a continuous and periodical function is needed, which is approximated by Fourier series.

For angle data of three joints, hip, knee and ankle from Winter's book (Winter 1991), the natural cadence is used. It is assumed that the right leg is symmetric to the left leg. The walking patterns are also symmetric, only a step behind. Therefore, data for the other leg can be obtained by shifting a step of the data from measurement. The curves are shown in Figure (3 - 2). Solid lines are from Winter's book; the dash lines are Fourier series. 

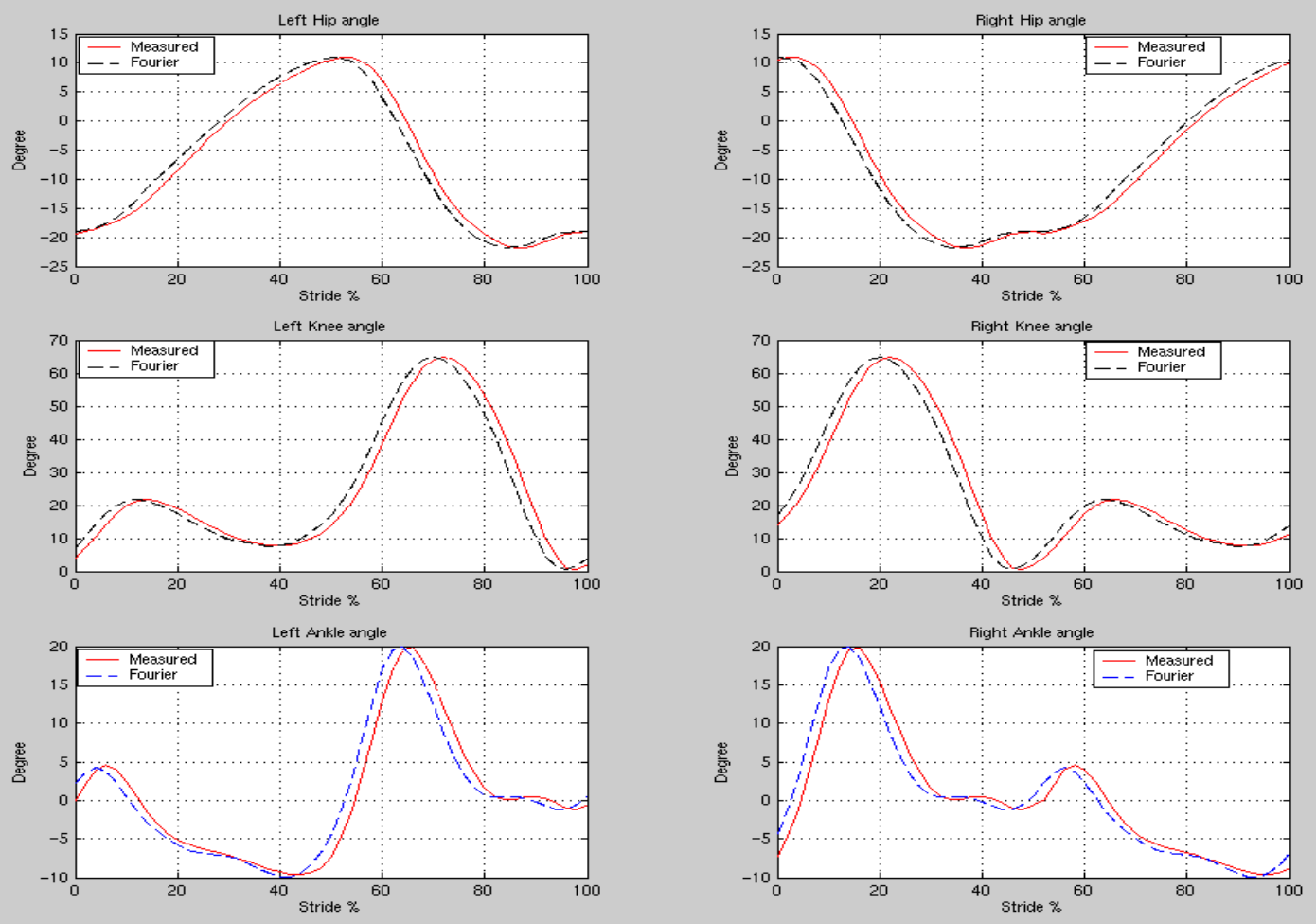

Figure (3 - 2) Fourier series and measured data of three joint angles

\subsection{Foot-Ground Contact Model}

The impact and friction effects are considered in the foot-ground contact model. The ground reaction forces (GRF) are approximated vertically by a linear viscoelastic model and horizontally by a pseudo-Coulomb friction model (Van den Bogert, 1989). Later a nonlinear viscoelastic model and distributed contact model are used to refine the model. 


\subsubsection{Linear Viscoelastic Model}

This model is used to calculate the impact force. Mathematically, the impact force is given as a function of position $(\mathrm{x}, \mathrm{y})$ and velocity of $(\dot{x}, \dot{y})$ of the ground contact point, which is allowed to penetrate into the ground.

$$
F_{y}=k y-\lambda_{v} \dot{y}, \text { when }(y \leq 0) ; F_{y}=0, \text { when }(\mathrm{y}>0)
$$

where, Fy is the impact force

In ADAMS, some refinements are needed. The force $F_{Z}$ is

IMPACT(Disp, Vel, Disp_trigger, K, Exp, C, Ramp_dist), substituted by letters

$\mathrm{F}_{\mathrm{Z}}=\operatorname{IMPACT}\left(\mathrm{q}, \dot{q}, \mathrm{q}_{1}, \mathrm{k}_{\mathrm{e}}, \mathrm{e}, \mathrm{C}_{\max }, \mathrm{d}\right)$, the true meaning of this function is

$$
\begin{aligned}
& \mathrm{F}_{\mathrm{Z}}=0, \text { if } q>q_{1} \\
& F_{z}=k\left(q_{1}-q\right)^{e}-C_{\text {max }} \dot{q} \times \operatorname{STEP}\left(q, q_{1}-d, 1, q_{1}, 0\right) \text {, if } q \leq q_{1}
\end{aligned}
$$

A large number of test simulations were performed to choose the appropriate value of $\mathrm{K}$ and Cmax. The values are: $\mathrm{k}=10000$ to $25000 \mathrm{~N} / \mathrm{m}, \mathrm{Cmax}=10$ to $1500 \mathrm{Ns} / \mathrm{m}$.

\subsubsection{Pseudo-Coulomb friction Model}

The typical Coulomb friction model introduces some discontinuities into the model, because it consists of two separate models for the stiction phase and for the sliding phase.

The pseudo-Coulomb friction model (Badoux 1964) was described as $F_{x}=-\gamma F_{y} \frac{\dot{x}}{|\dot{x}|}$, where Fx is the horizontal component of a GRF and $\gamma$ is the coefficient of friction.

Because $\dot{x}$ may be zero, which will cause numerical singularity, the model was approxi- 
mated by $F_{x}=-\frac{2 \gamma F_{y}}{\pi} \operatorname{atan}\left(\frac{\lambda_{h} \pi}{2 \gamma F_{y}} \dot{x}\right)$. but it still causes numerical singularity in

ADAMS simulation, for Fy is zero during the swinging phase. A little change is made as below:

$$
F_{x}=-\gamma F_{y} \frac{\dot{x}}{|\dot{x}|+\varepsilon},
$$

In three dimension,

$$
F_{x}=-\gamma F_{z} \frac{\dot{x}}{\sqrt{\dot{x}^{2}+\dot{y}^{2}}+\varepsilon}, F_{y}=-\gamma F_{z} \frac{\dot{y}}{\sqrt{\dot{x}^{2}+\dot{y}^{2}}+\varepsilon}
$$

where $\varepsilon$ is small constant value to ensure that denominator is not zero.

The value of these coefficients are: $\gamma=0.3, \varepsilon=1 * 10^{-5} \mathrm{~m} / \mathrm{s}$

\subsubsection{Contact points}

The contact points shown in Figure (3 - 3) are distributed along the foot with elastic and damping coefficients changed. The reason to use the distributed contact points is to minimize the penetration of the feet and to maximally approximate the simulation results to the measurements. This contact points plan comes up after many attempts with two contact points in which the simulated ground reaction force failed to agree with the measurements.

The other reason to change the elastic and damping coefficients is the changed foot pressure (Perry 1992). From Perry's book we can see the pressure distribution. For a nonlinear spring, different deformation will give a different elastic coefficient. 


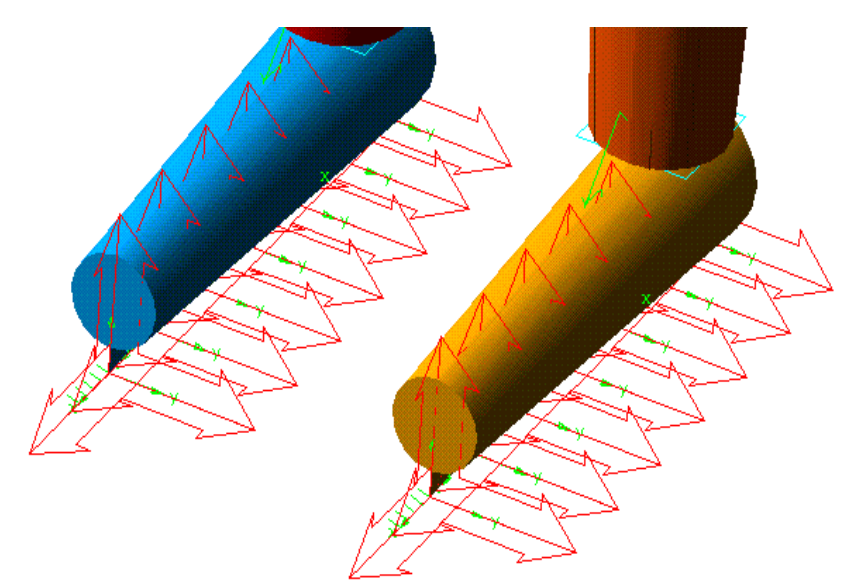

Figure (3 - 3) Contact points

\subsection{Upper Body Balance Control}

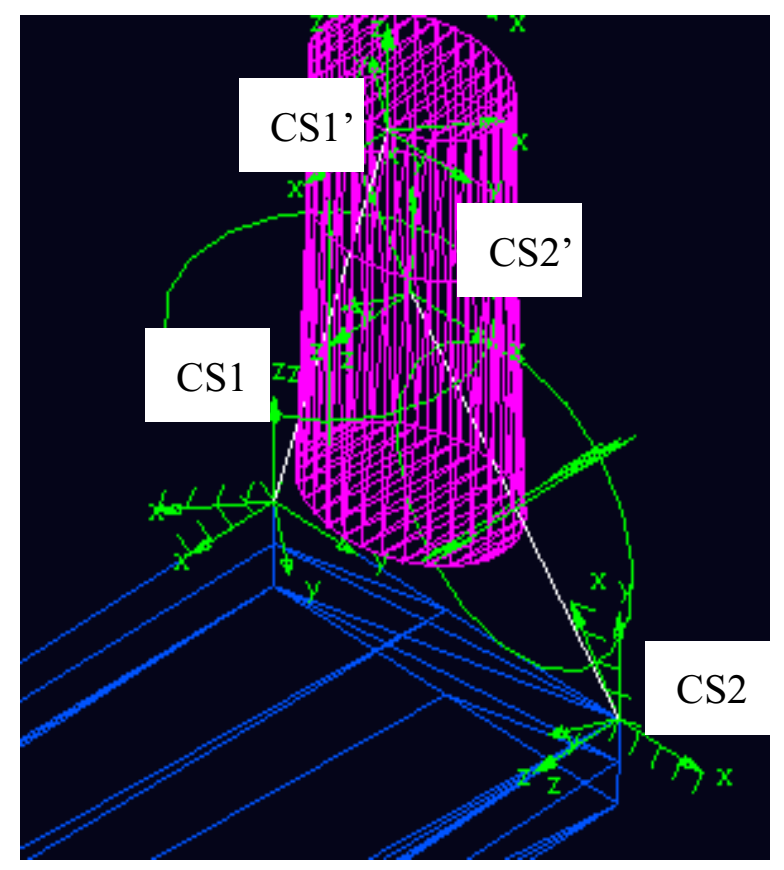

Figure (3 - 4) Primitive joints

Before the model is ready for simulation, a way must be found to control the balance of upper body. Two primitive joints, Parallel axes (two white lines shown in Figure (3 - 
4)), are added between the torso and the ground to constrain 3 rotational DOFs. The $Z$ axes of CS1 and CS1' are parallel and perpendicular to the ground. It leaves the body to rotate on $\mathrm{Z}$ axis. Then the parallel $\mathrm{Z}$ axes of CS2 and CS2' are added to constrain the left freedom, because $\mathrm{Z}$ axes of CS2 and CS2' are in the walking direction and perpendicular to the $\mathrm{Z}$ axes of $\mathrm{CS} 1$ and $\mathrm{CS} 1$ '. It is possible to use three control torques in three axes to constrain the 3 rotational DOFs. However, it is difficult to find the right coefficients in torque Eqn (3-5).

$$
T=k\left(\theta_{\text {desired }}-\theta_{\text {actual }}\right)+k_{\lambda}\left(\dot{\theta}_{\text {desired }}-\dot{\theta}_{\text {actual }}\right)
$$

Where $\theta_{\text {desired }}$ is the desired angle of the upper body to any axis, $\theta_{\text {actual }}$ is the actual angle of the upper body to any axis.

Compared to this method, adding two joints is much easier.The fully constrained model is shown in Figure (3 - 5). 


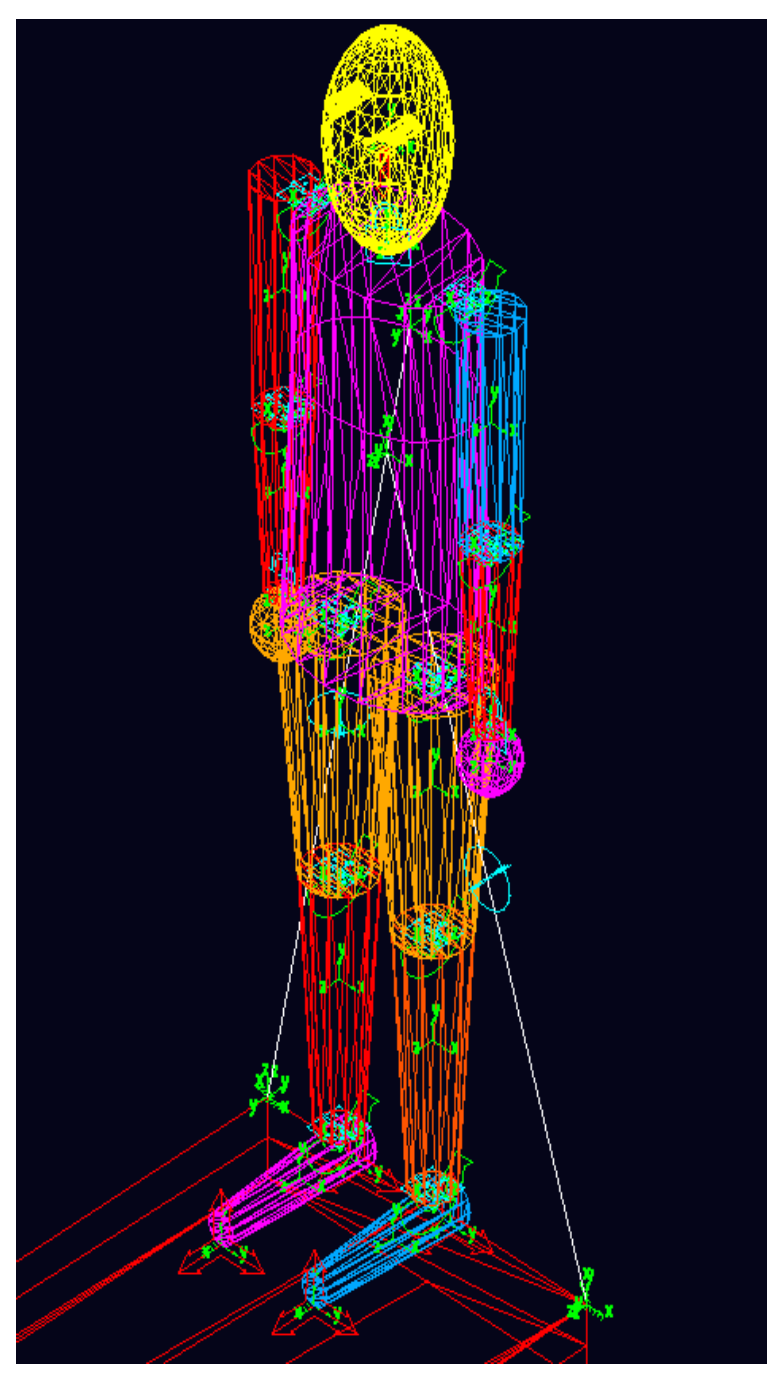

Figure (3 - 5) Constrained human model

\subsection{Analysis of Simulation Results}

To validate the model, we need to compare the results of simulation with those measured data described in Chapter 2. Simulation is executed in ADAMS. From the animation shown in Figure (3 - 6), we can observe sliding on stance foot and penetrations of both feet to the ground, which are directly derived from the pseudo-Coulomb friction model and 
linear viscoelastic model respectively. The following section examines the results in detail.



Figure (3 - 6) Simulation of human walking

\subsubsection{Ground Reaction Force}

Ground reaction forces results directly from foot ground contact model. Compared with measured forces (Winter 1991), the simulated forces can show us the accuracy of the ground contact model. By changing any of the values of $\mathrm{K}$ and $\mathrm{C}$ in Eqn (3-2), $\gamma$ in Eqn (34), the position of contact points or contact model itself (from linear to nonlinear) the ground reaction forces will vary. Sometimes there are small change in forces, but it may change other parameters greatly. That is why at the same time the ground reaction forces are checked other parameters are also checked to ensure the model is reliable or reasonable.

Obtaining appropriate values for coefficients $\mathrm{K}$ and $\mathrm{C}$ in Eqn (3-2) is time consuming. If initial $\mathrm{K}$ is estimated on the weight divided by the desired penetration, then the model may jump off the floor, and $\mathrm{C}$ must be added to adjust. Of course, the values other authors 
provided in their models which were shown in Chapter 1 can provide an initial guess.

After many tries, simulation results were obtained and will be shown in the remaining parts of this chapter. For details regarding $\mathrm{K}$ and $\mathrm{C}$, please see Adams model.

As previously stated, the contact points under the foot are distributed along the foot in the sagittal plane. The ground reaction forces shown in Figure (3 - 7) are the sum of forces from all contact points on one foot.



Figure (3 - 7) Ground reaction force

From the figure, the following conclusions were made:

- Impact force, top on Figure (3 - 7), agrees with the measurement very well. The maximum force from measurement is $795.27 \mathrm{~N}$. The maximum force from simulation is $849.5 \mathrm{~N}$, which is $6.8 \%$ higher than the measured one. That is a very close result. 
- The stance phase can be measured from initial touching point to last touching point on the stance foot, or the duration of impact force. The calculated stance phase is $60 \%$ of the gait cycle, while the measured is $64 \%$ of the cycle.

- Between two impact forces, there is one small touching force. This is the direct result of the penetration of the stance foot. Because of the penetration of the stance leg, the clearance for swing leg is not enough during the middle swing. The swing foot will bump the ground. From the simulation the exact case can be observed.

- Large error can be found in progressional force (bottom one on Figure (3 - 7)), especially the first step. As previously stated, the motion assigned for each joint is from steady walking, but the first step is a transition from resting to steady walking. There is no initial dropping velocity like that in steady walking and no braking period, only propulsion. That's why in this transitional period there is no backward force. This serves as a reminder that the dynamic simulation is different from kinematic simulation. A non zero initial movement to a joint can be assigned, but this movement depends on force. It is easy to be numerical singular for the force or acceleration. The denominator for acceleration is almost zero. That is why in Adams simulation a large non zero initial movement can not be assigned. To solve this problem, a STEP function can be added or multiplied to make the initial movement be zero or close to zero. It only affects the results for a short period with the STEP function, but has no affect on the remaining simulation.

- The progressional force of second stride looks better than that of the first stride. But the problem here is that on heel strike there has a forward force, that means not braking first. The direct cause can be seen from Figure (3 - 8). While the 
touching force declines to zero there is the largest sliding velocity on stance foot.

This results in the whole body moving backward. That is why some sliding on that position is observed during the simulation. The sliding is derived from friction model and will be explored in next part.

- The closest progressional force is propulsion force. The maximum measured force is $161 \mathrm{~N}$. The maximum calculated force is $187 \mathrm{~N}, 16 \%$ over measured data, and the shape looks very alike. The largest error is from braking force.

\subsubsection{Sliding and Penetration}

The sliding is shown in Figure (3 - 8). Let's look at the second stride. The force, displacement and velocity are from the same foot.

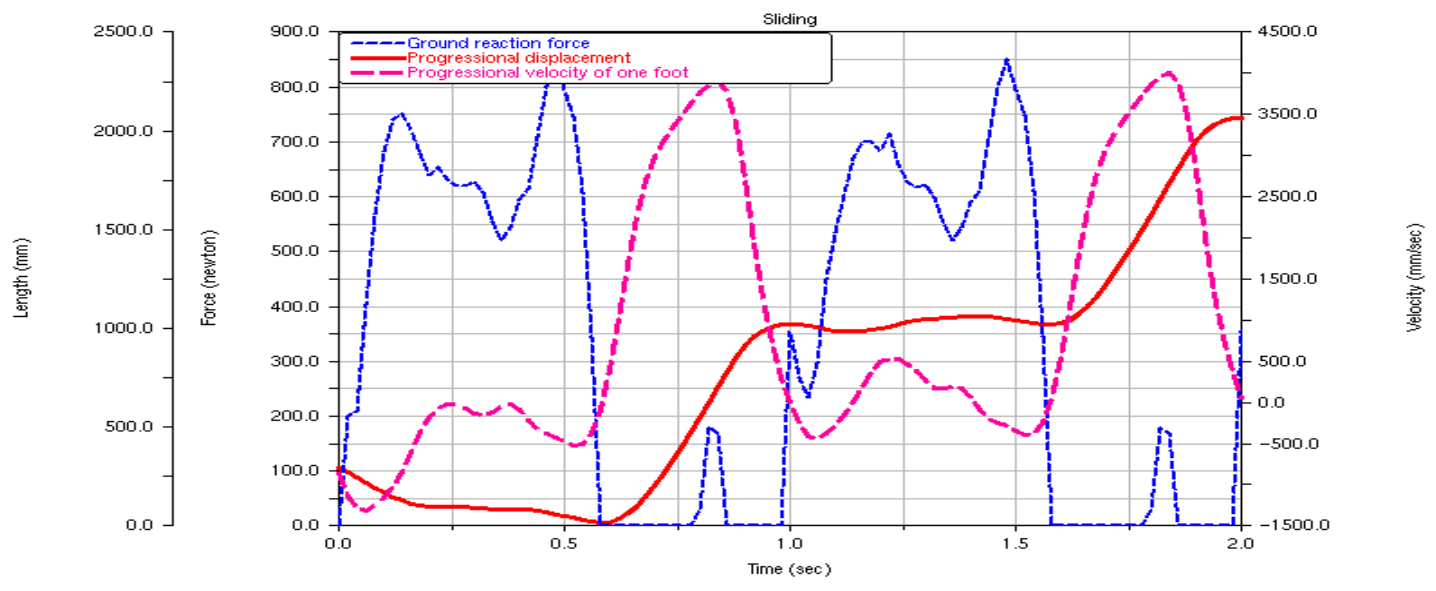

Figure (3 - 8) Sliding of normal walking

It can be seen that during stance phase (non zero force), the velocity is not zero. The reason for this can be explained directly from friction model. In Eqn (3-3), the Pseudo friction is 


$$
\mu=-\gamma \frac{\dot{x}}{|\dot{x}|+\varepsilon}
$$

It is shown in Figure (3 - 9), where $\gamma=0.3, \varepsilon=1 / 100000$. When $\dot{x}=0, \mu=0$, that means $F_{x}=0$. This is not true for static period. The true friction is also shown in Figure (3 - 9). Where

$$
\mu=-\mu_{0} \text { to } \mu_{0} \text {, when } \dot{x}=0 \text {, i.e. } \mathrm{F}_{\mathrm{x}}=-\mu_{0} * \mathrm{~F}_{\mathrm{y}} \text { to } \mu_{0} * \mathrm{~F}_{\mathrm{y}}
$$

where $\mu_{0}$ is the static friction.

Although the friction force of some value is there, the true friction value can not be determined from Eqn (3-7), but it can be calculated from force balance equation, or Newton's second law.

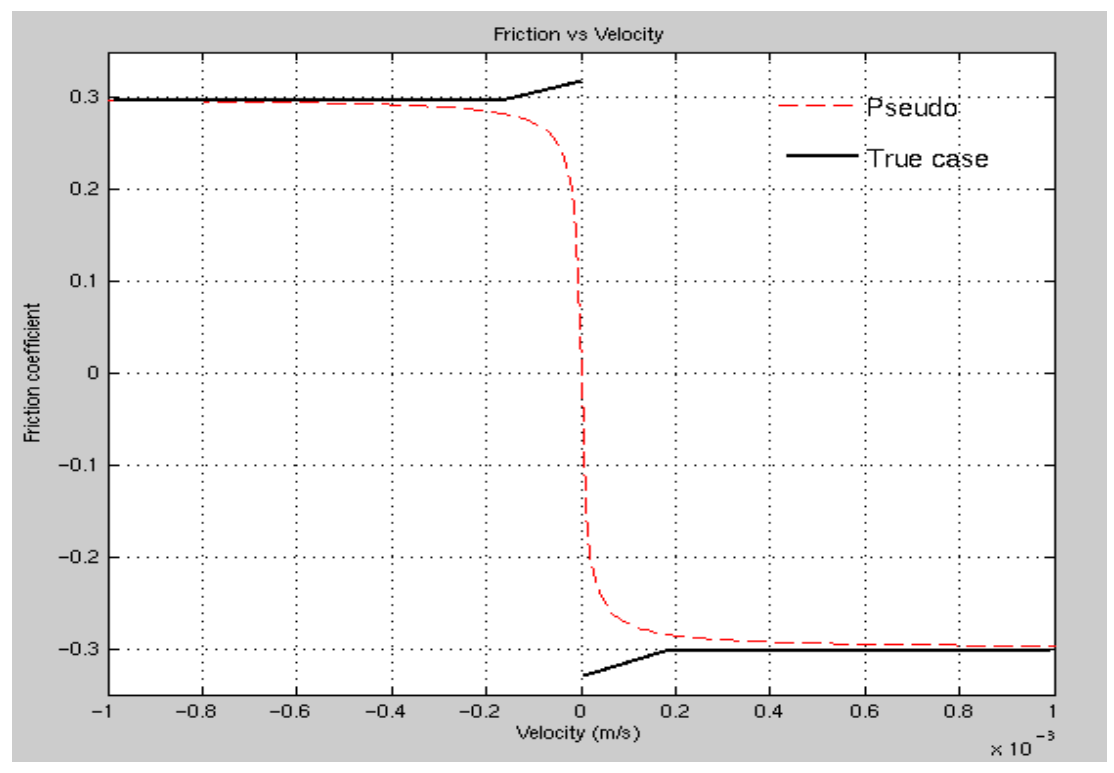

Figure (3 - 9) Friction comparison

In other words, from Eqn (3-6), if $\dot{x}=0$, then $\mathrm{F}_{\mathrm{X}}=0$, that means the force on stance foot is zero because of no movement. However, if there is no force on stance foot how can 
movement occur? Force is the cause; movement is the result. It also means that for movement to occur, the stance foot must move in opposite direction to pick up force to move the whole body. This is not true for real walking, because during the static period, force is picked up from $-\mu_{0} * \mathrm{~F}_{\mathrm{y}}$ to $\mu_{0} * \mathrm{~F}_{\mathrm{y}}$. Summed up, the sliding is inevitable and necessary. Minimizing slide is a question to be answered for the future. Maybe some change in the model for static period can be made. The motions for all joints were assigned, why not use $\mathrm{F}=$ ma to simulate the force? If this equation is used, how to deal with double support? If the contact period can be divided into static and sliding period that may solve the double support problem. From Figure (3 - 7) the calculated force in sliding period agrees very well with that of measured one. Is this force so important? Later it can be observed that this force will have large affects on joint moment and power consumption.

From the combined areas of vertical force, foot displacement and velocity in progressional direction, the maximum sliding speed is $0.43 \mathrm{~m} / \mathrm{s}$, the whole sliding displacement during the stance is $0 \mathrm{~mm}$, displacement on contact force ending point $(1.0196 \mathrm{~m})$ minus that of starting point $(1.0196 \mathrm{~m})$, and the stride is $1.0429 \mathrm{~m}$, displacement on force starting point $(2.0625 \mathrm{~m})$ minus that on last ending point $(1.0196 \mathrm{~m})$.

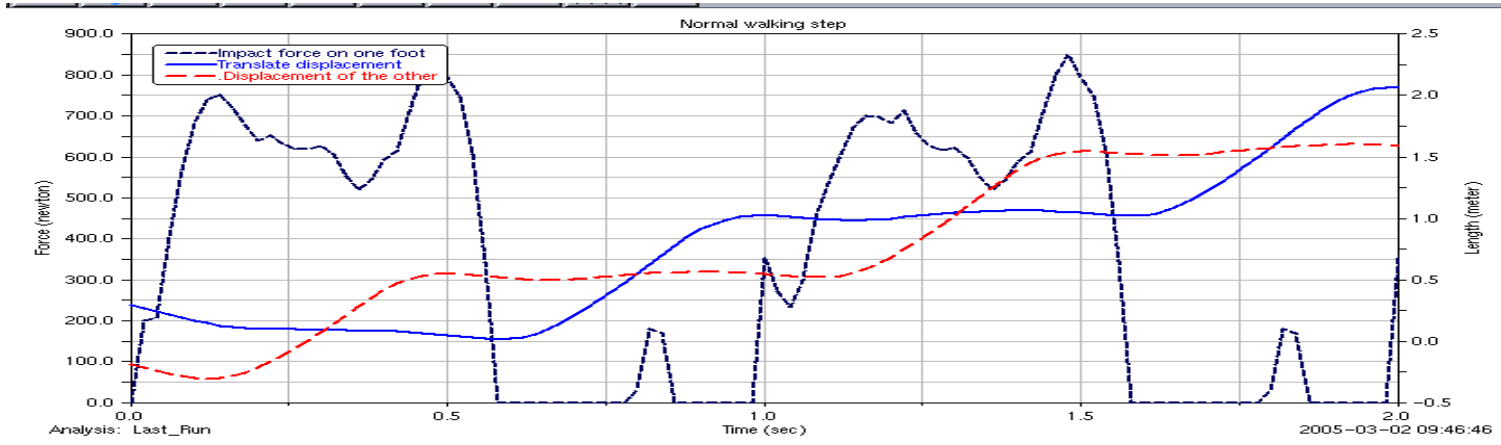

Figure (3 - 10) Step length of normal walking 
Step length can be more accurate from Figure (3 - 10). The step length at the heel strike is $0.4673 \mathrm{~m}$. It can also be obtained from toe off, but the displacement of both toes is needed to be plotted out. Because it is measured at the same moment from the figure, it does not include the affect of sliding.

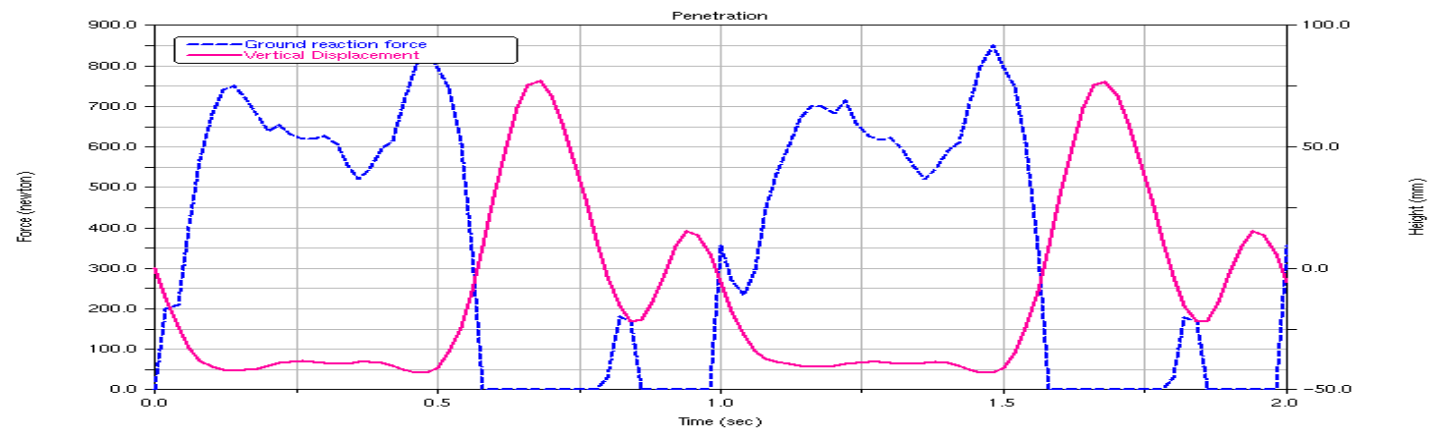

Figure (3 - 11) Penetration of normal walking

The penetration shown in Figure (3 - 11) is resulted from impact model. If there is no penetration, then there is no force, although there is no undefined force problem like friction in static period. Thus penetration is also necessary, just make it as small as possible to decrease its affects on the swing leg. Because the penetration in stance leg will decrease the clearance of the swing leg, this will make the swing foot bump into the ground in middle swing. That is why there is force in swing phase, as seen in Figure (3 - 11).

From the combined areas of vertical force and foot displacement in vertical direction, assuming the displacement of the initial position is zero, the maximum penetration is 43 $\mathrm{mm}$.

\subsubsection{The Center of Mass}

Now the displacement of center of mass (COM) in sagittal plane shown in Figure (3 12) will be examined. There is no measured data to compare. The shape looks like a sinu- 
soidal curve. The total rising and falling is $21.9 \mathrm{~mm}$. The initial position (double support) is supposed to be the lowest point during the walking. The displacement below this point is the affects of the penetration on the COM. It is $7.6 \mathrm{~mm}$.

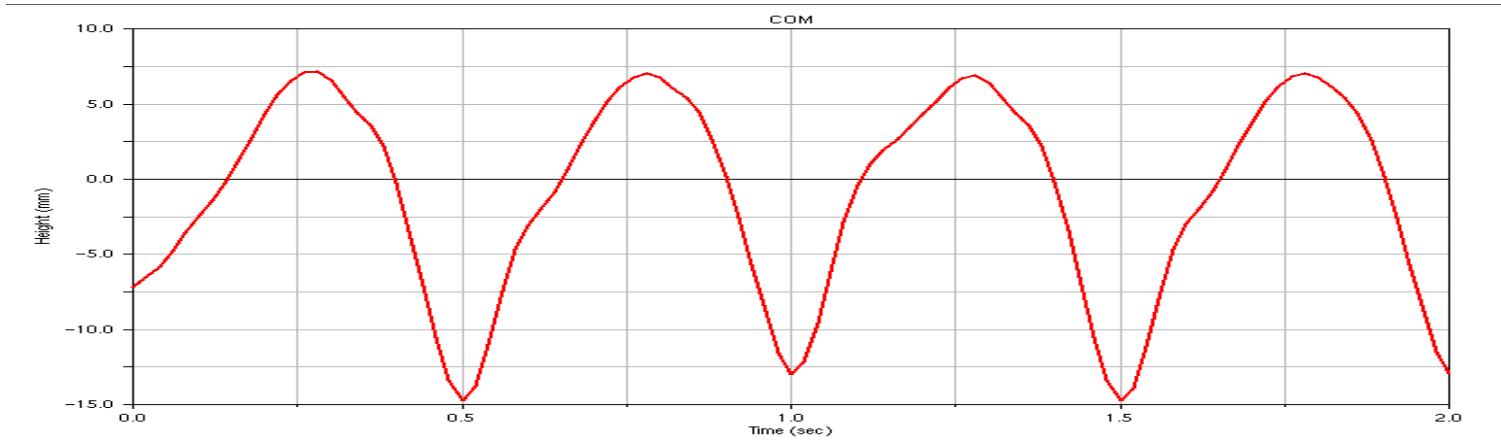

Figure (3 - 12) Displacement of COM

\subsubsection{Joint Motions}

As previously stated, joint angles are used as driving power in the simulation. The simulated results are the exact input. The only errors of these input are from Fourier series. At most 9 items of the whole Fourier series were used. The joint angles are shown in Figure (3 - 13). The curves for the other leg just shift half the cycle. 


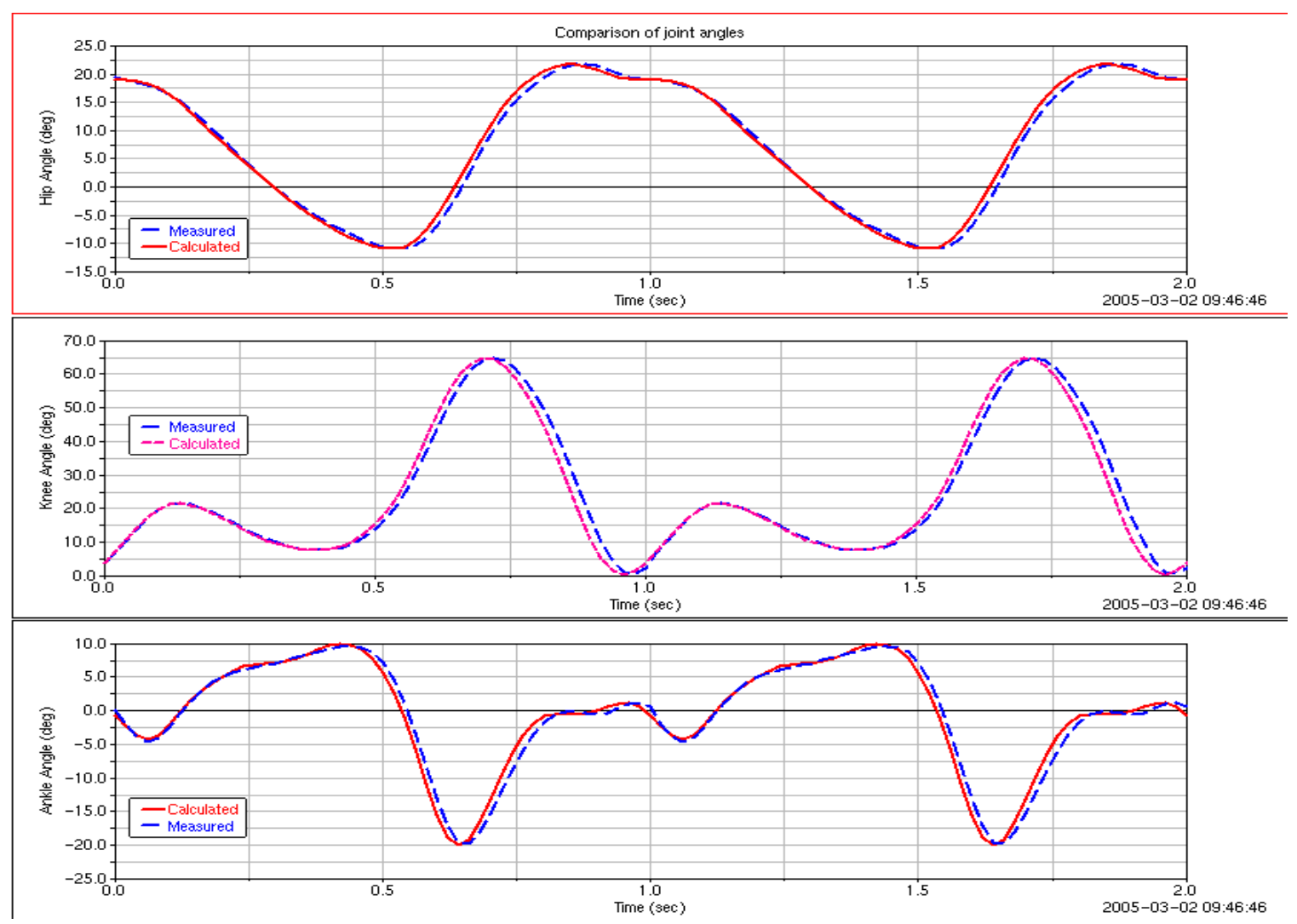

Figure (3 - 13) Angles of three joints

\subsubsection{Joint Moment and Power Consumption}

The torque and power required to drive hip joint, knee joint and ankle joint are shown in Figure (3 - 14), Figure (3 - 15) and Figure (3 - 16) respectively. The second stride, steady walking is examined. The first is transitional. Looking at the shapes of the calculated moment and power, they looks like the shape of friction. As previously stated, the moment equals force time the length of arm. The longer the arm, the bigger the moment. Therefore, the friction has the biggest affect on the hip joint, because of the longest arm. The moment and power of ankle joint agrees very well with the measurements. The max- 
imum and the average moment and power are summed up as shown in Table 3 - 2 on page 52 .

The total power for both legs are shown in Figure (3 - 17). Only power for one leg was known, then shift a step to get the power for the other leg. The total measured power for both legs were added together, which can be compared with the simulated power. Their shape looks alike, but values have large errors. The average for one leg in the table conflicts with the total average of three joints. In order to compare steady walking, the first step was removed. However, this was not comparable to the other simulation. Therefore the data including the first step was still needed. Then the average power for one leg is $56.2 \mathrm{~W}$.

In Chapter 2, mechanical power estimation was $77.5 \mathrm{~W}$. Therefore the simulation results are creditable.

Table (3 - 2): Calculated and Measured Power and Moment in Normal Walking

\begin{tabular}{l|l|l|l|l|l|l|l|l}
\hline \multirow{2}{*}{ Joints } & \multicolumn{4}{|c|}{ Torque (Nm) } & \multicolumn{4}{c}{ Power (W) } \\
\cline { 2 - 9 } & \multicolumn{2}{|c|}{ Calculated } & \multicolumn{2}{c|}{ Measured } & \multicolumn{2}{c}{ Calculated } & \multicolumn{2}{c}{ Measured } \\
\cline { 2 - 9 } & Max & Ave & Max & Ave & Max & Ave & Max & Ave \\
\hline Hip & 179.68 & 27.6 & 44.1 & -1.9 & 290 & 41.6 & 52.4 & 5.9 \\
\hline Knee & 87 & 17 & 45.2 & 1.3 & 213 & 8 & 54.8 & -10.87 \\
\hline Ankle & 106.6 & 33.78 & 119.7 & 32.5 & 265 & 6.6 & 244.8 & 14.7 \\
\hline One leg (First step in account) & & & 56.2 & & \\
\hline One leg (First step not included) & & 343 & 40 & 236 & 9.8 \\
\hline
\end{tabular}





Figure (3 - 14) Torque and power for hip
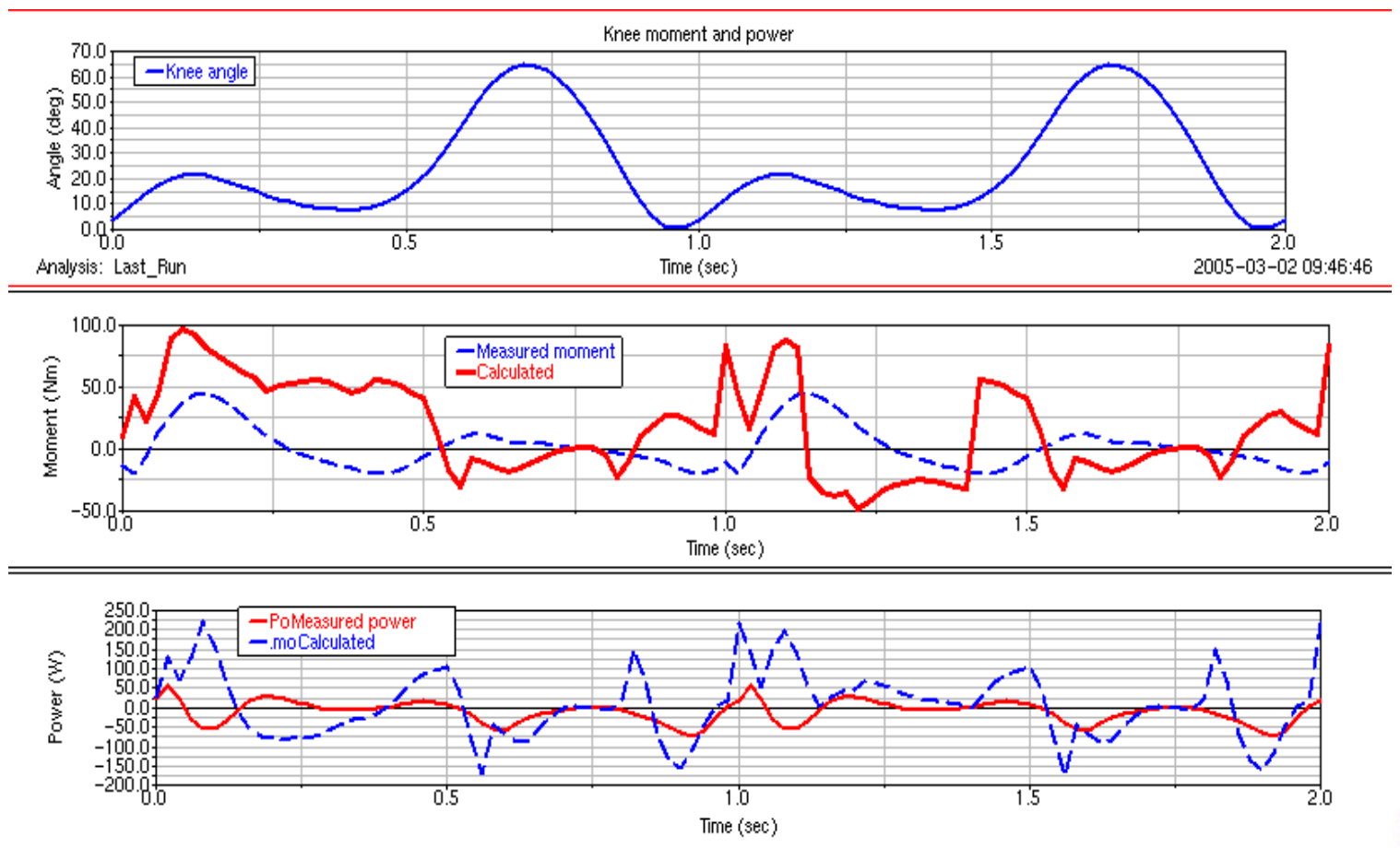

Figure (3 - 15) Torque and power for knee 

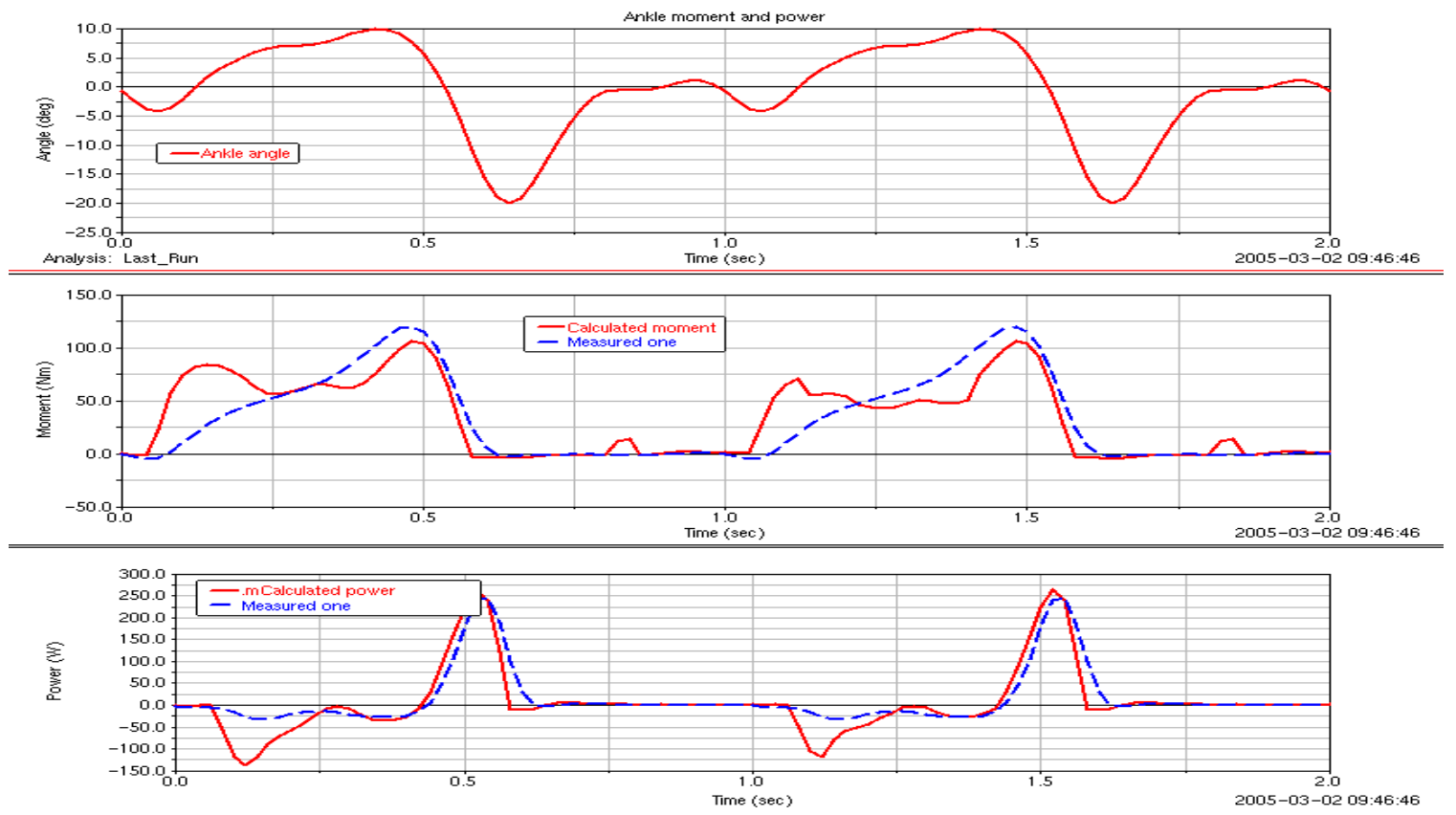

Figure (3 - 16) Torque and power for ankle

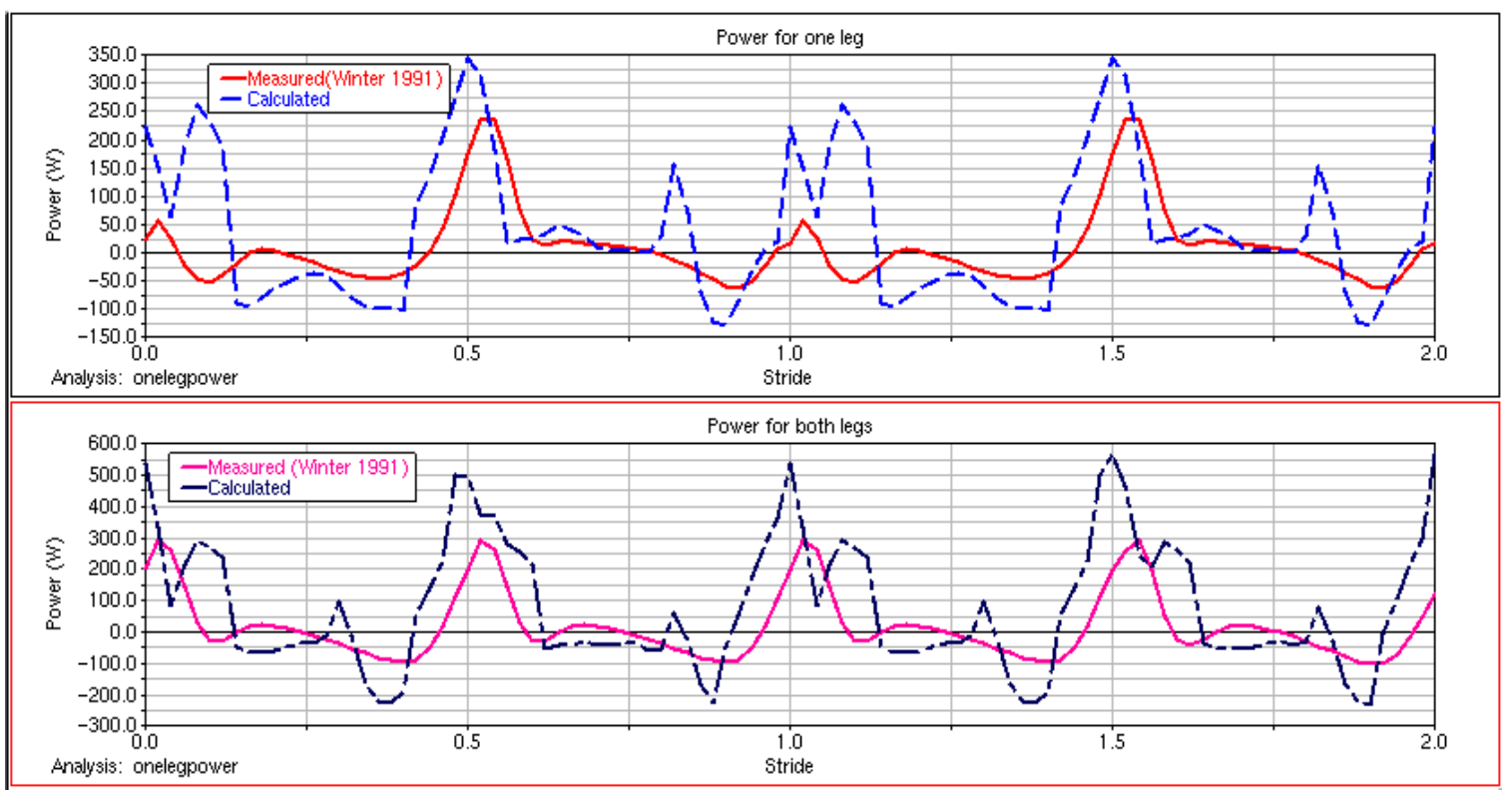

Figure (3 - 17) Power for normal walking 


\subsubsection{Control Torque}

As stated, two parallel axes joints were used to control the upper body's balance. They worked together to constrain 3 rotational DOF. The torque shown in Figure $(3-18)$ are the sum of these two joints respectively in three directions. Torque in the sagittal plane is the largest; its cycle is a step, $50 \%$ of a stride. Which leg is swinging does not matter. Torque in coronal plane is the second, and torque in transverse plane is the smallest. The curve shape of each one looks the same but in opposite directions. That means which leg is in swing matters here. This makes sense for human symmetric to the sagittal plane.The maximum, minimum and average values are shown inTable 3 - 3 on page 55 .

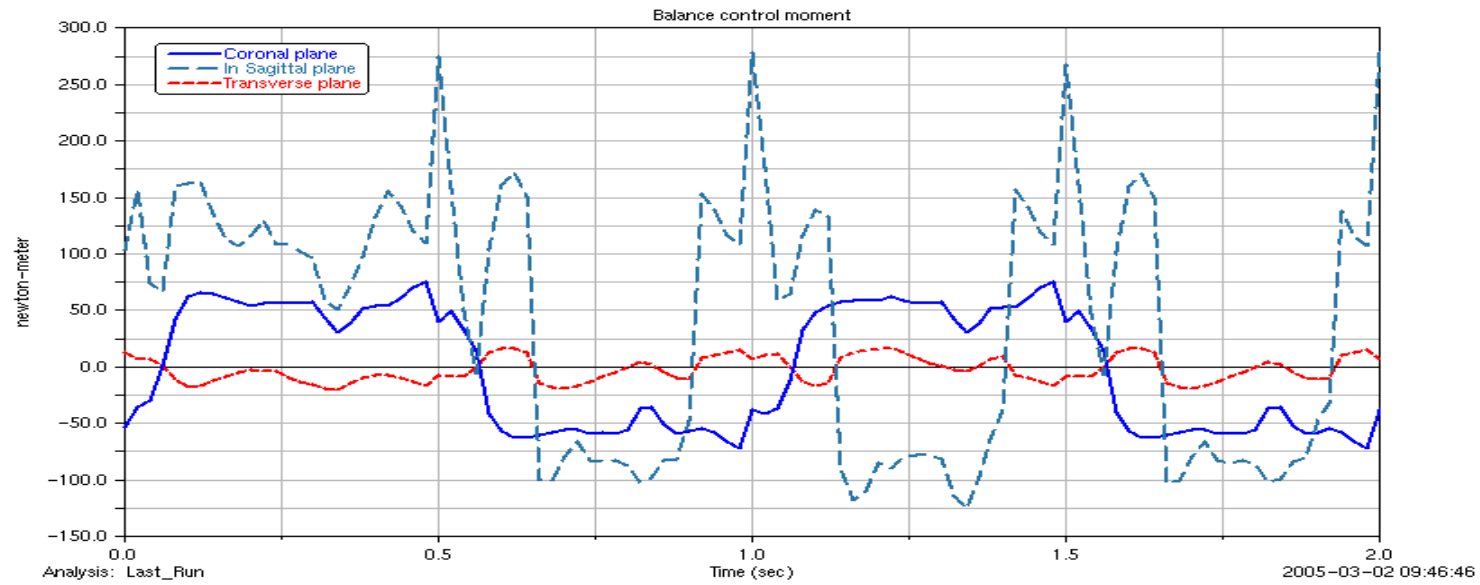

Figure (3 - 18) Balance control moment

Table (3 - 3): Control Torque of Normal Walking

\begin{tabular}{|c|c|c|c|}
\hline Plane & Maximum $(\mathrm{Nm})$ & Minimum $(\mathrm{Nm})$ & Average $(\mathrm{Nm})$ \\
\hline \hline Sagittal & 279.5 & -124.9 & 40.9 \\
\hline Coronal & 75.53 & -72.18 & -0.5 \\
\hline Transverse & 17 & -19.6 & -2.4 \\
\hline
\end{tabular}




\subsection{Summary}

The simulated results were compared with measured data to validate the model. The result from impact model agreed well with the measured. That is, impact model and contact points distribution are reliable. Friction model resulted in sliding, but the total sliding displacement was zero. This model did result in some error in progressional force, which had affects on joint moments and power consumption. The construction of a new friction model was proposed. 


\section{SIMULATION OF CURRENT PGO PHYSICAL MODEL}

\subsection{Current PGO Model}

The current PGO shown in Figure (4 - 1) is a one degree of freedom system for each leg with both hip and knee motion coupled. The coupling gears are not shown in the figure.

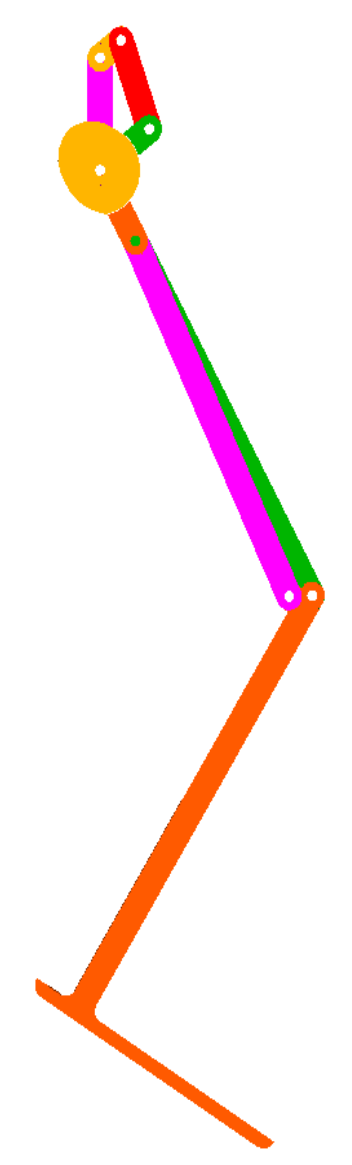

Figure (4 - 1) PGO model 
The motion of the hip joint is approximated with a four-bar mechanism and the motion of the knee with a cam-modulated slider crank mechanism. The cam profile is machined into the face of the lower gear which is driven by the upper gear, the crank of the four-bar mechanism. The lower gear in turn drives the cam-modulated linkage. Since the cam follower is captured in a slotted cam profile, it always remains in contact with the cam during flexion or extension.

A cam profile can be derived from the normal knee joint angle function. Thus the same knee function as in the human model is set in knee joint to simulate the model first to get the correct cam profile. This is accomplished by creating Trace Spline on the cam, the cam running in opposite direction to the crank of four-bar mechanism. Then the correct cam profile is used to make a cam follower joint to drive the knee joint and at the same time the knee function is deactivated.

The hip function and knee function generated by these two mechanism of the PGO are shown in Figure (4 - 2). The hip function of the PGO shows a significant difference from that of normal walking, while both PGO and normal knee functions are exactly the same as shown in Figure (4 - 3). 


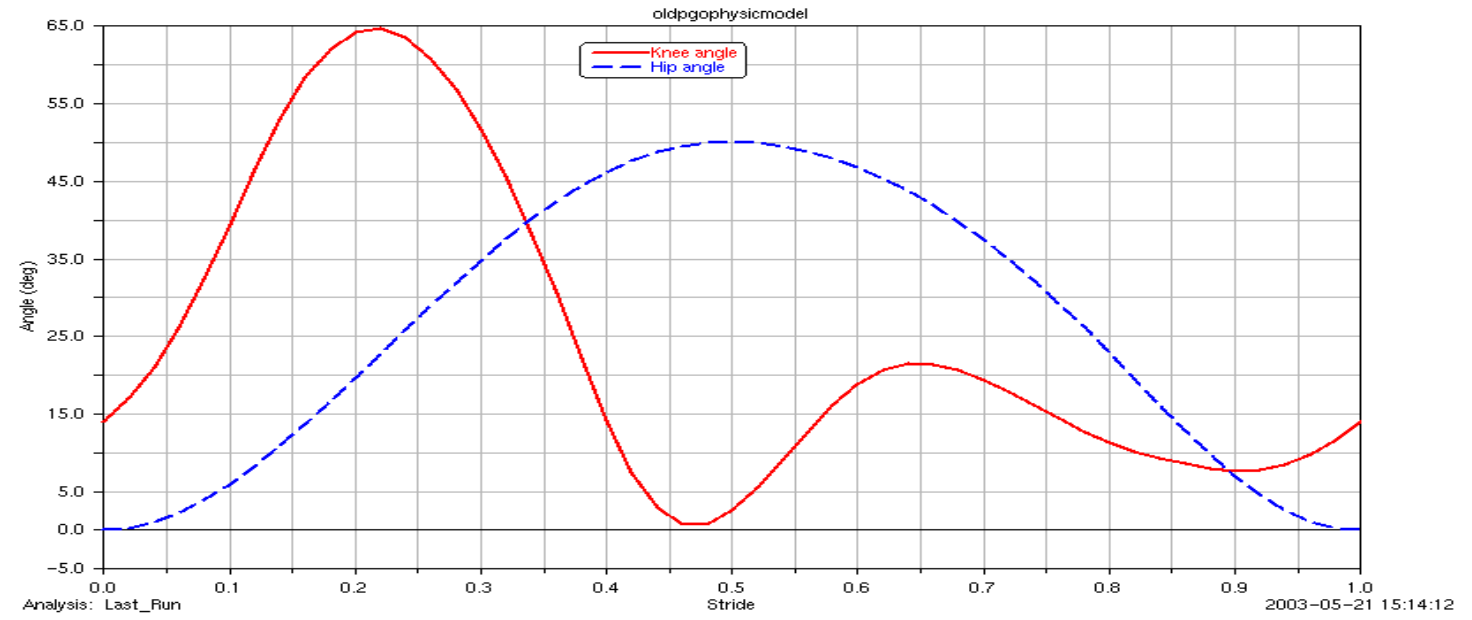

Figure (4 - 2) Joint angles of PGO

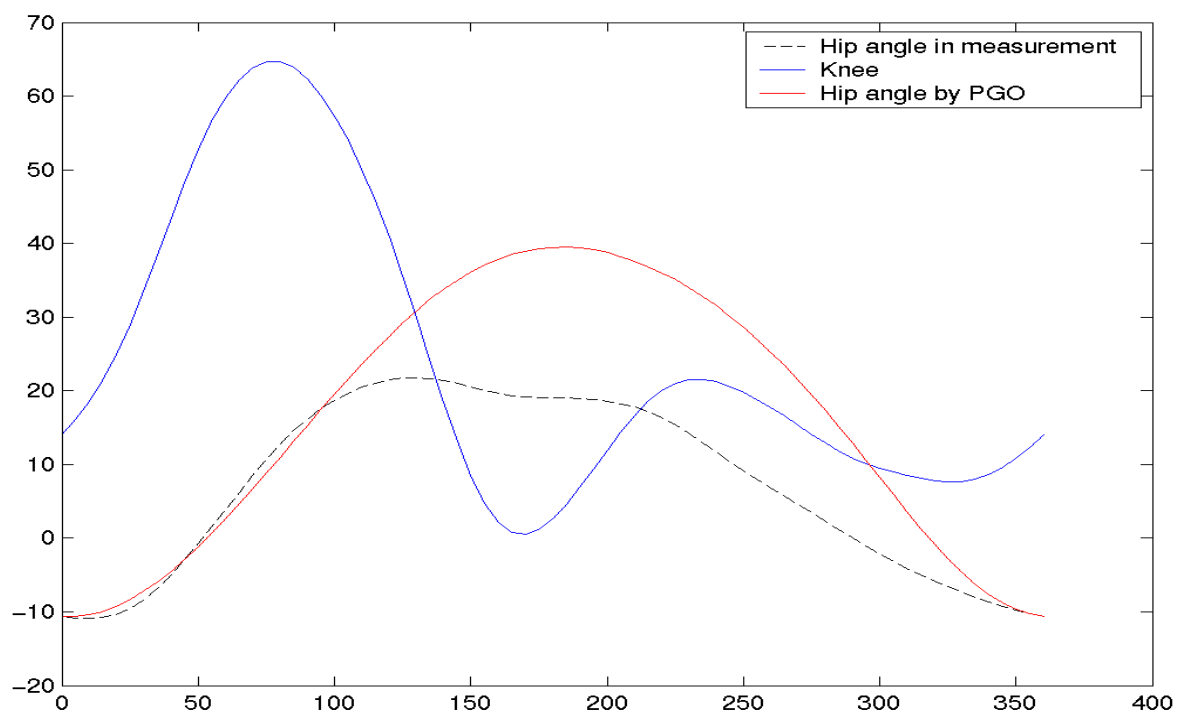

Figure (4 - 3) Comparison of hip and knee angles of PGO with those of human walking 


\subsection{Human in Current PGO Model}

The model above is just one leg of the PGO. The previous research was more focused on single leg, like the design of four-bar mechanism and cam-modulated slider crank mechanism, time ratio and strength of the PGO (Ruthenberg, 1997). Here a patient walking in the PGO was simulated to fully investigate the function of the entire device including both legs. Full understanding of the function of the whole device will make the requirement for single leg or single part clearer. That will make the single part to easily satisfy the demand of the entire function. The PGO walking model is shown in Figure (4 4). The hip joints of human model are in line with those of the PGO, and deactivated. The knee joints of the human model are also deactivated, and they are not in the line with those of the PGO, because the length of the thigh of human model is not the same as that of the PGO. The whole weight of the human model is supported by the PGO. 


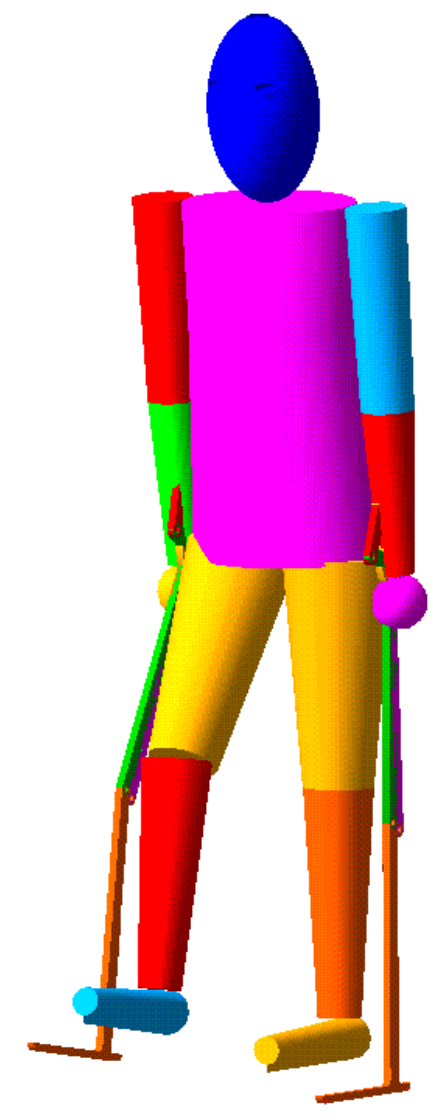

Figure (4 - 4) Human in PGO

\subsection{Joint Motion Function}

The hip joint of the PGO is powered by a DC motor through a four-bar mechanism. It seems simple just to input a steady rotation function for the crank. In fact, only one leg moves at a time, so the rotation function is not continuous. This is the problem to execute the simulation, so Fourier series is also used to form a continuous motion function. For the sake of accuracy of Fourier transformation, the velocity, not the angle of the crank is the input (Both were tried, but the angle resulted in large errors in the simulation, especially 
for the initial condition. This caused the model to jump or walk backward in the simulation). The curves are shown in Figure (4 - 5).

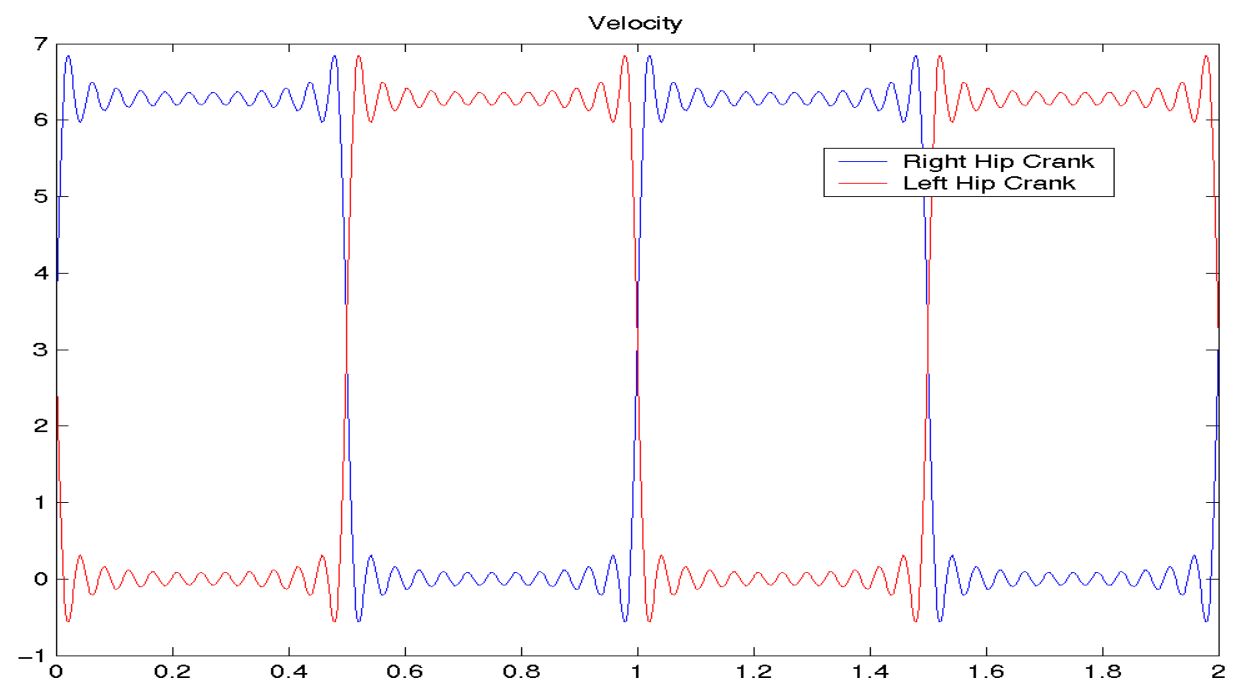

Figure (4 - 5) Fourier series of motor rotation speed of PGO

\subsection{Analysis of Simulation Results}

The foot-ground contact model and the joints to control the upper body's balance validated in Chapter 3 were used here. The shoulder and elbow joints were fixed because in real walking the arms were used to control the upper body's balance. Upper body was 10 degree leaned forward. Otherwise the swing leg can not touch the ground. The simulation was shown in Figure (4 - 6). To find the real causes of poor function of the current PGO, the simulation of walking in PGO was compared with the simulation of human walking. The results corresponding to Chapter 3 were shown below. 


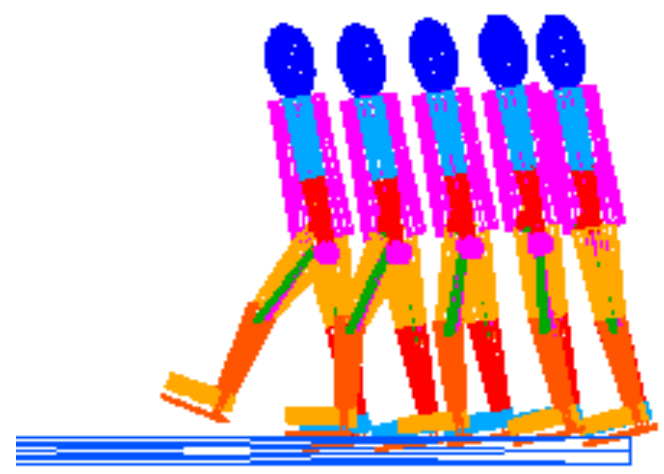

Figure (4 - 6) Simulation of PGO walking

\subsubsection{Ground Reaction Force}

Compared with the simulation of normal walking, the vertical reaction force shown in Figure $(4-7)$ has three peaks. This is different from normal walking which has only two peaks, and the value is much higher than normal walking. The contact model may bring larger error in the simulation of PGO walking. If measured ground reaction force of PGO walking were obtained, the contact model could be refined. But here the same contact model is used as that used in the simulation of normal walking as a basis for comparison.

The maximum impact force is $1587.7 \mathrm{~N}, 86 \%$ over normal walking, of course the weight now is the sum of human model and PGO. The stance phase is $64 \%$ of gait cycle, and the double support shown in the bottom plot of Figure (4 - 7) is $14 \%$ of gait cycle. Because larger error in progressional force even in the simulation of normal walking, here the values are not compared. The force is shown in Figure (4 - 8). This will aid an understanding why there is a big sliding after the heel strike. 


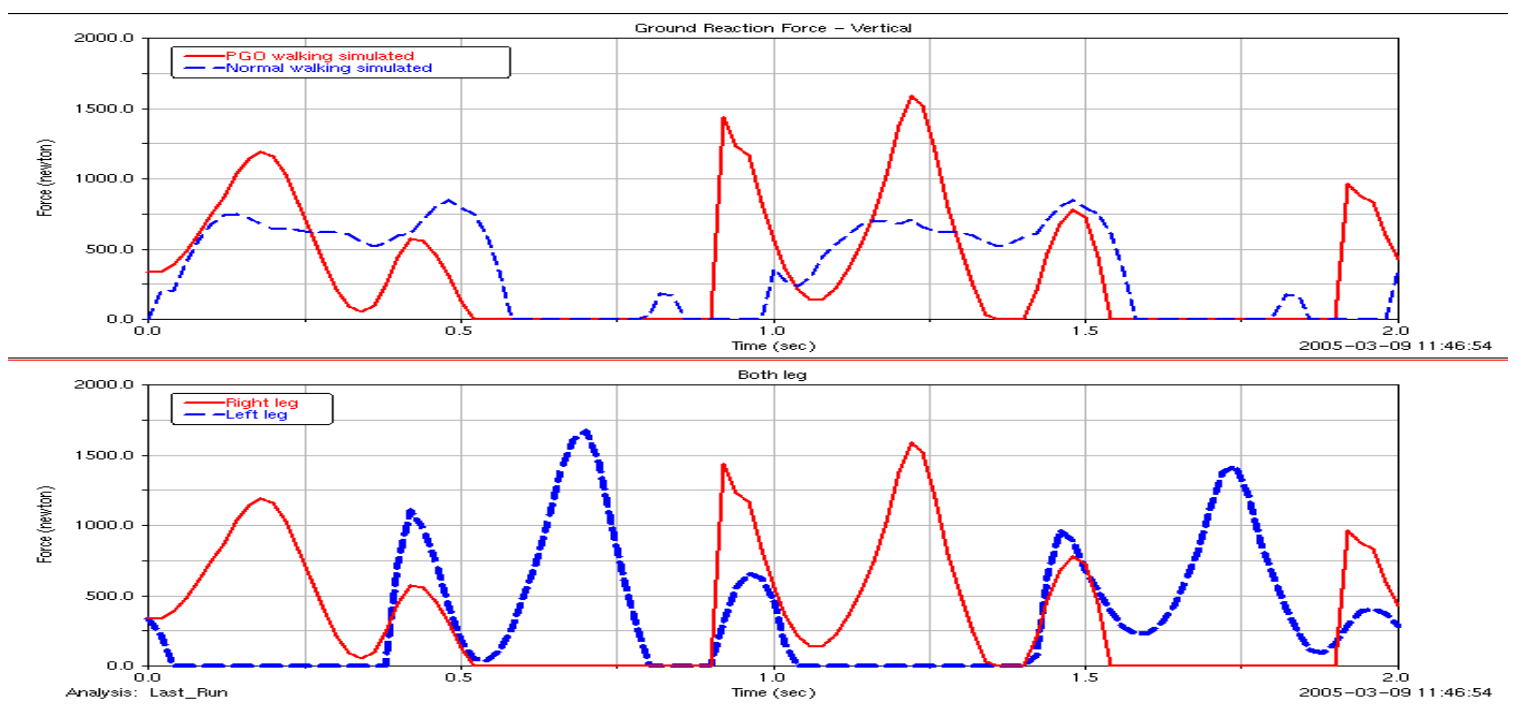

Figure (4 - 7) Vertical ground reaction force in PGO walking

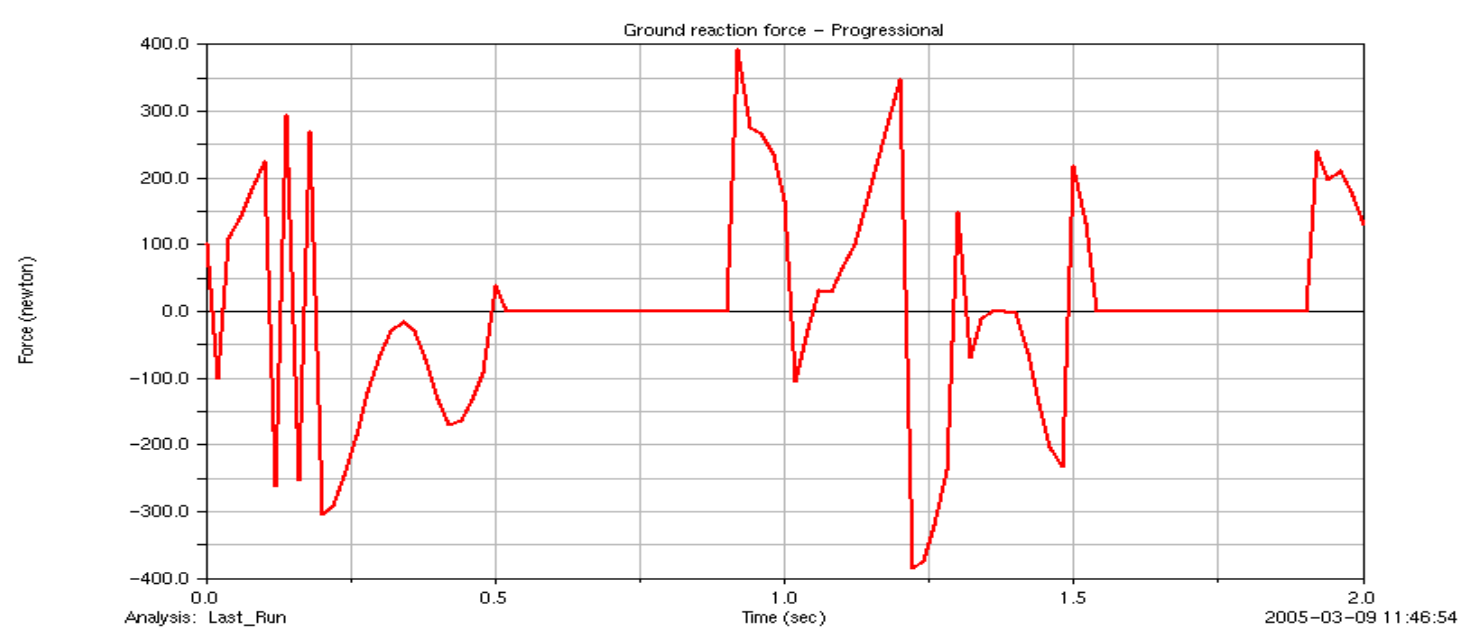

Figure (4 - 8) Progressional force in PGO walking

\subsubsection{Sliding and Penetration}

The sliding is shown in Figure (4 - 9). Compared with Figure (3 - 8), on heel strike, the starting point of impact force, the progressional velocity is $0.3 \mathrm{~m} / \mathrm{s}$. During stance 
phase velocity changed back and forth, but the total sliding displacement is zero. In Figure (4 - 9), on heel strike, the progressional velocity is $-1.1 \mathrm{~m} / \mathrm{s}$. That is why after heel strike the stance foot is drawn backward $0.26 \mathrm{~m}$. For real PGO walking this is not true. As seen from the tape that after the heel strike, no power acts on that foot. The power is switched to the trailing leg, to try to bring the trailing leg to the front of that stance leg. The problem here is that without changing the position of stance leg it is hard to bring the trailing leg to the front. In normal walking the motion on stance leg is to reposition the stance leg and the position of COM. The walking depends on the force of the stance leg. Because no power works on stance leg, it seems that the device just moved swing leg. That is why it is hard to move forward.

If the power on the stance foot is like the simulation, why it can not move the trailing body forward instead of drawing the stance leg backward? From time period 0.9 to $1 \mathrm{~s}$ in Figure (4 - 9), after the heel strike, there is the double support. Both feet have forces on them until both feet reach the same position. That means no initial swing and no clearance for the trailing foot to move forward. From the curve of progressional displacement in Figure (4 - 9), at the same time trailing body moves forward, the stance leg is drawn back. Because weight is larger for trailing body, it moves slower. That is why the sliding of the stance leg is seen.

If the upper body does not lean forward, there is no movement forward. The step length on heel strike depends on the leaning of upper body.

In normal walking, after heel strike there is also a double support, but after the double support there is pre-swing and initial swing. The trailing body has power to make clearance to bring it to or over the stance leg. 
The penetration is shown in Figure (4 - 10). The penetration is $38.6 \mathrm{~mm}$.

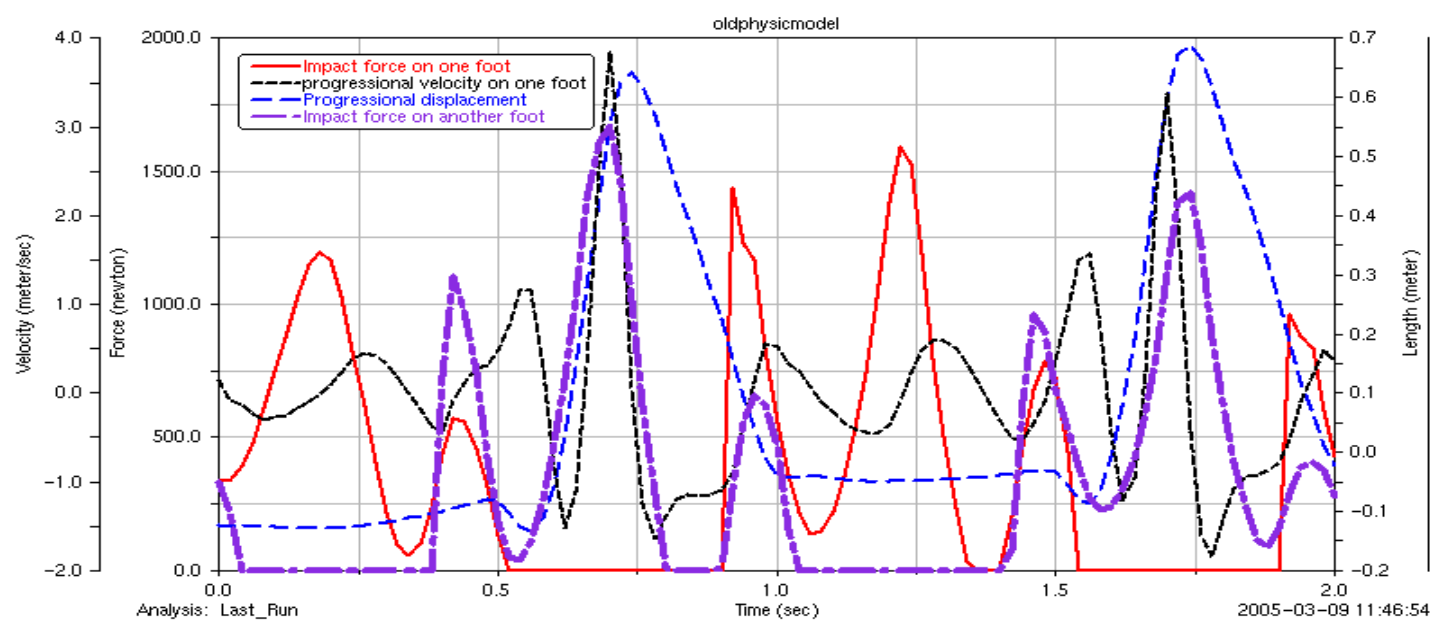

Figure (4 - 9) Sliding of PGO walking

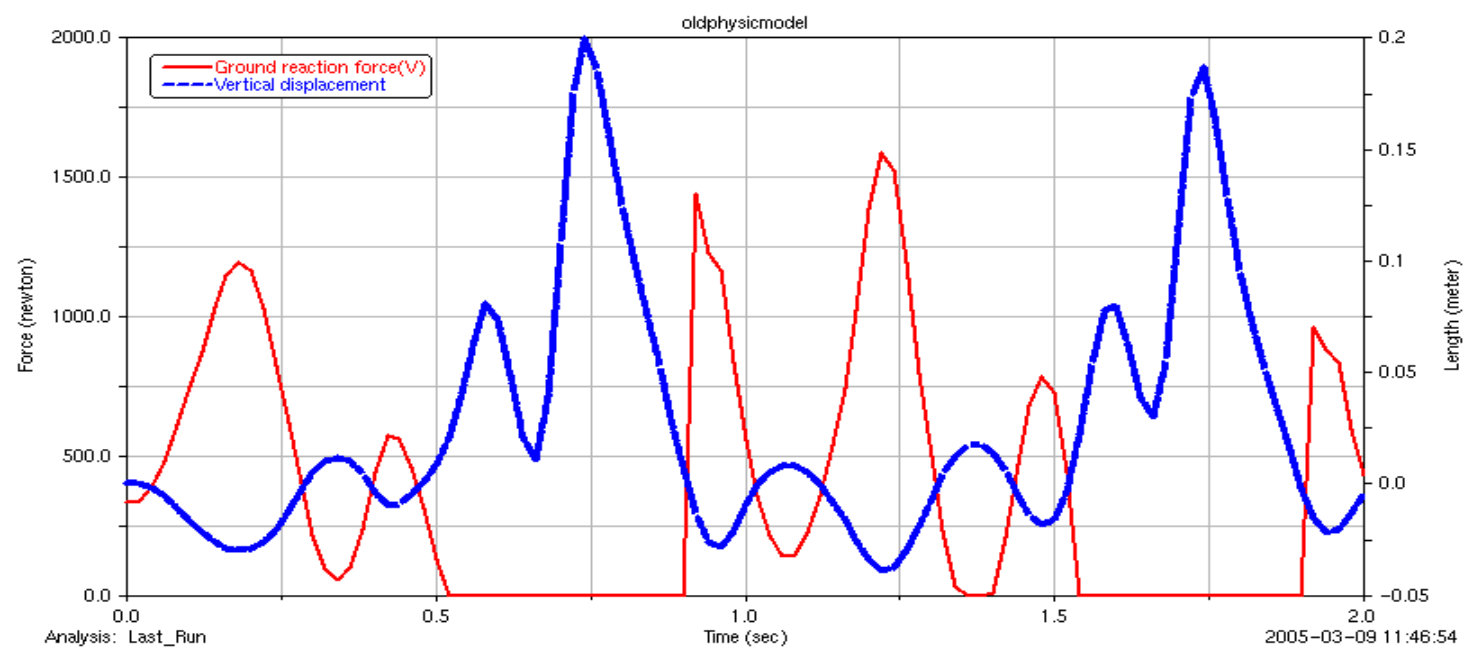

Figure (4 - 10) Penetration of PGO walking

\subsubsection{The Center of Mass}

The normal walking COM line in Figure (4 - 11) is the line in Figure (3 - 12) shift half step to adjust them to start in the same position. Now we can see the displacement in PGO 
walking is bigger than in normal walking. That also suggests that the same contact model may cause some error in PGO walking.

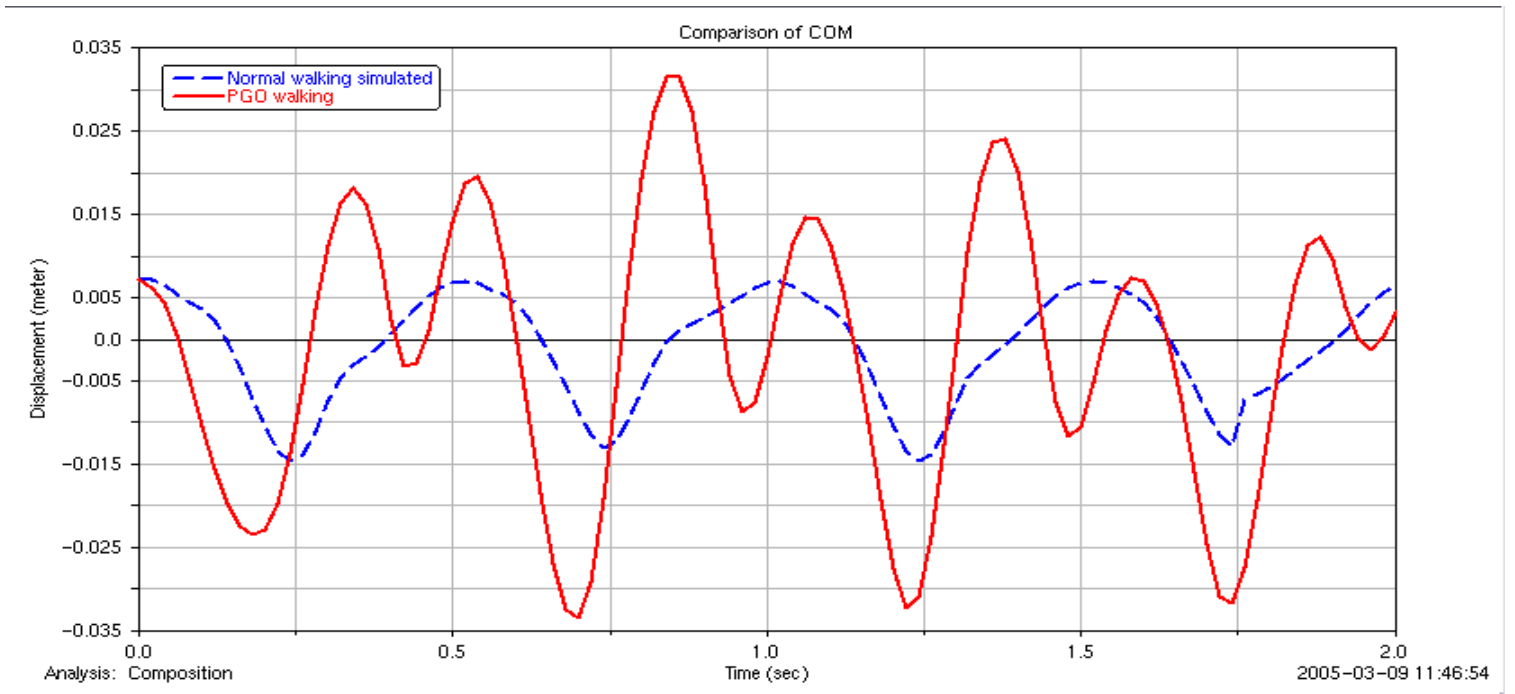

Figure (4 - 11) Displacement of COM in PGO walking

\subsubsection{Joint Motions}

Hip joint and knee joint angles are shown in Figure (4 - 12). Red solid lines are from measured normal walking, and the blue dash lines are the simulation of PGO walking. Although the joint functions of one leg of the PGO in Figure (4 - 3) looks very close to those of normal walking, but when put two legs together we will find a big difference between them. As far as the whole cycle is concerned, they are not similar any more.

- The joints only work in half gait cycle

- No negative angle for hip joint; no extension for the hip

The main cause is that only one leg moves at a time. If we change it to move both legs at the same time, there is problem for hip joint. It will need a negative angle. With hip and 
knee coupled it can not get standing position. This will be explained in detail in later chapter.
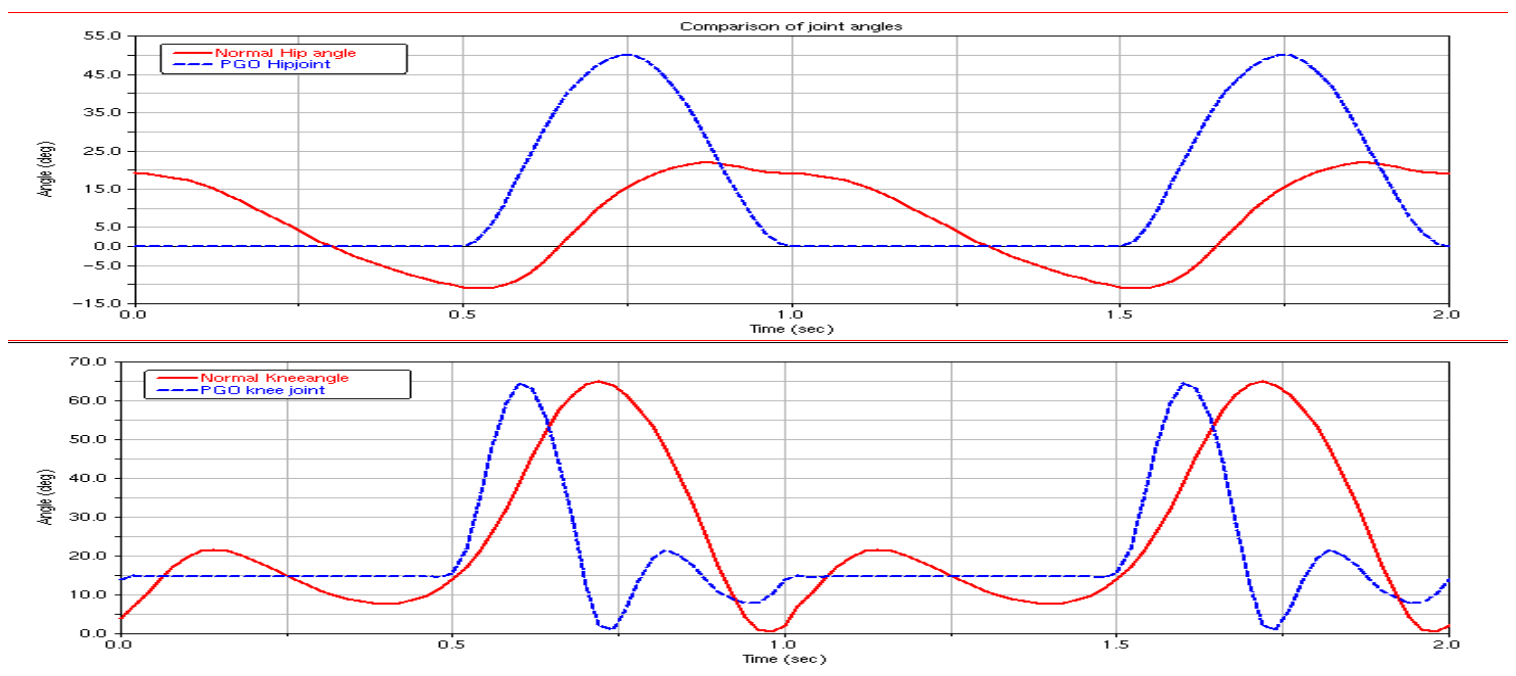

Figure (4 - 12) Hip and knee angles in PGO walking

\subsubsection{Joint Torque and Power Consumption}

The joint torque and power consumption are shown in Figure (4 - 13) and Figure (4 14). The total power consumption is in Figure (4 - 15). The maximum and average torque and power excerpted from these figures are shown in Table $4-1$ on page 68 .

Table (4 - 1): Torque and Power of Current PGO

\begin{tabular}{c|c|c|c|c}
\hline \multirow{2}{*}{ Joints } & \multicolumn{2}{|c|}{ Torque (Nm) } & \multicolumn{2}{c}{ Power (W) } \\
\cline { 2 - 5 } & Maximum & Average & Maximum & Average \\
\hline Hip & 128.5 & 12 & 1660 & 135 \\
\hline Knee & 120 & 14.7 & 515 & -50 \\
\hline \multicolumn{3}{c}{ Total (hip + knee) } & 1507 & 85 \\
\hline
\end{tabular}






Figure (4 - 13) Torque and power of hip joint in PGO walking

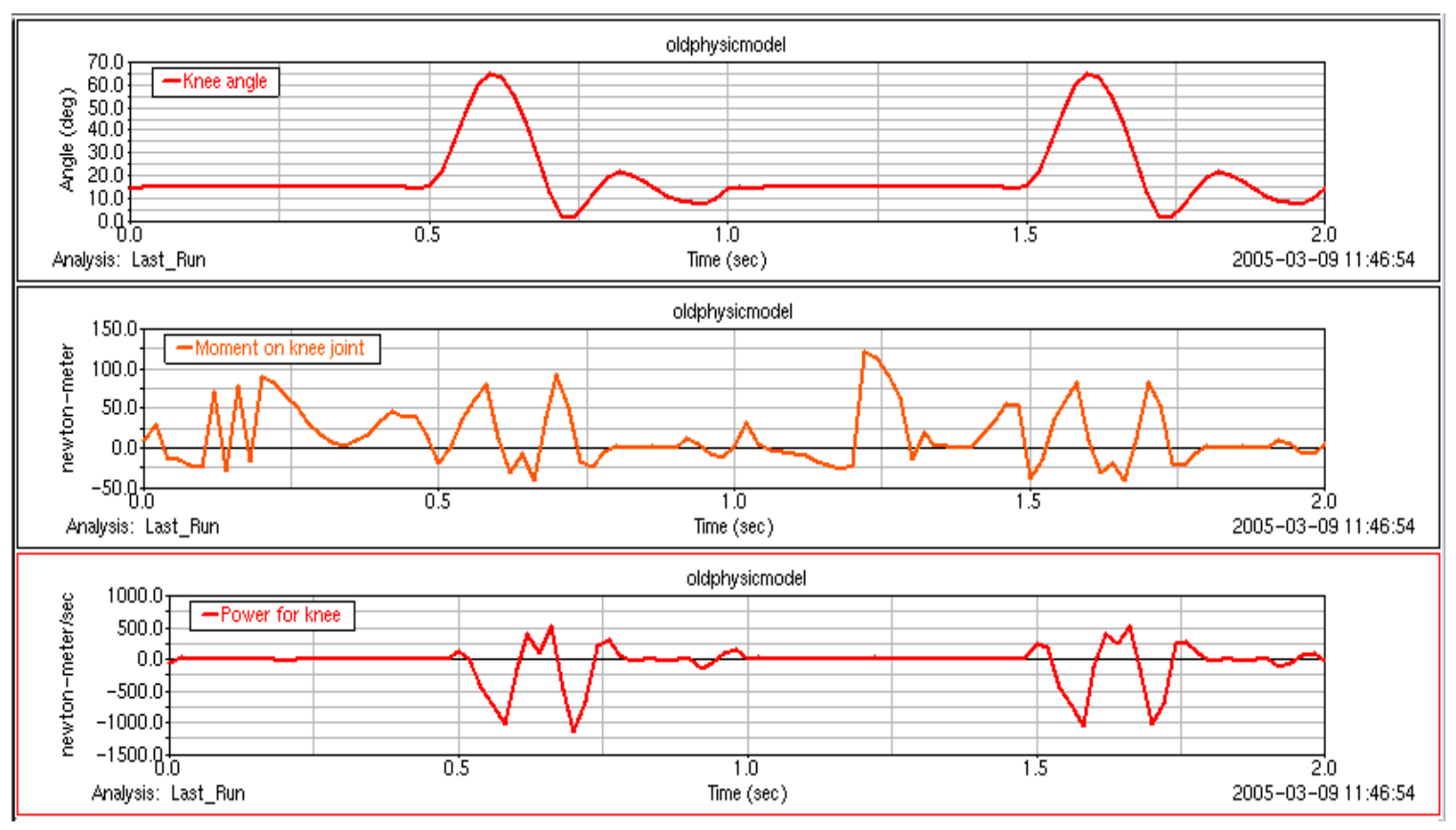

Figure (4 - 14) Torque and power of knee joint in PGO walking 


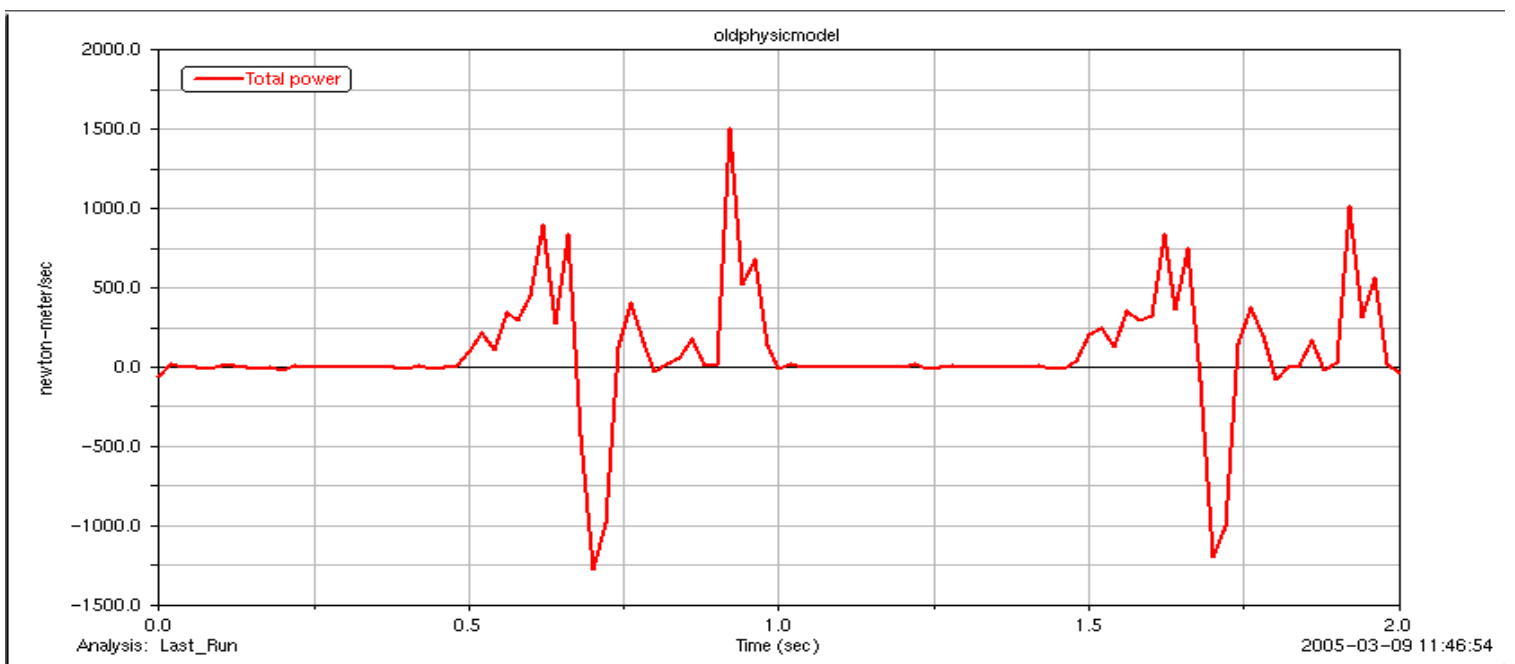

Figure (4 - 15) Total power of hip and knee in PGO walking

\subsubsection{Control Torque}

The balance control torque is shown in Figure (4 - 16). Because the body leans forward, most of the torque in the sagittal plane is used to balance the torque of weight. The data from the figure is shown in Table 4 - 2 on page 71. 


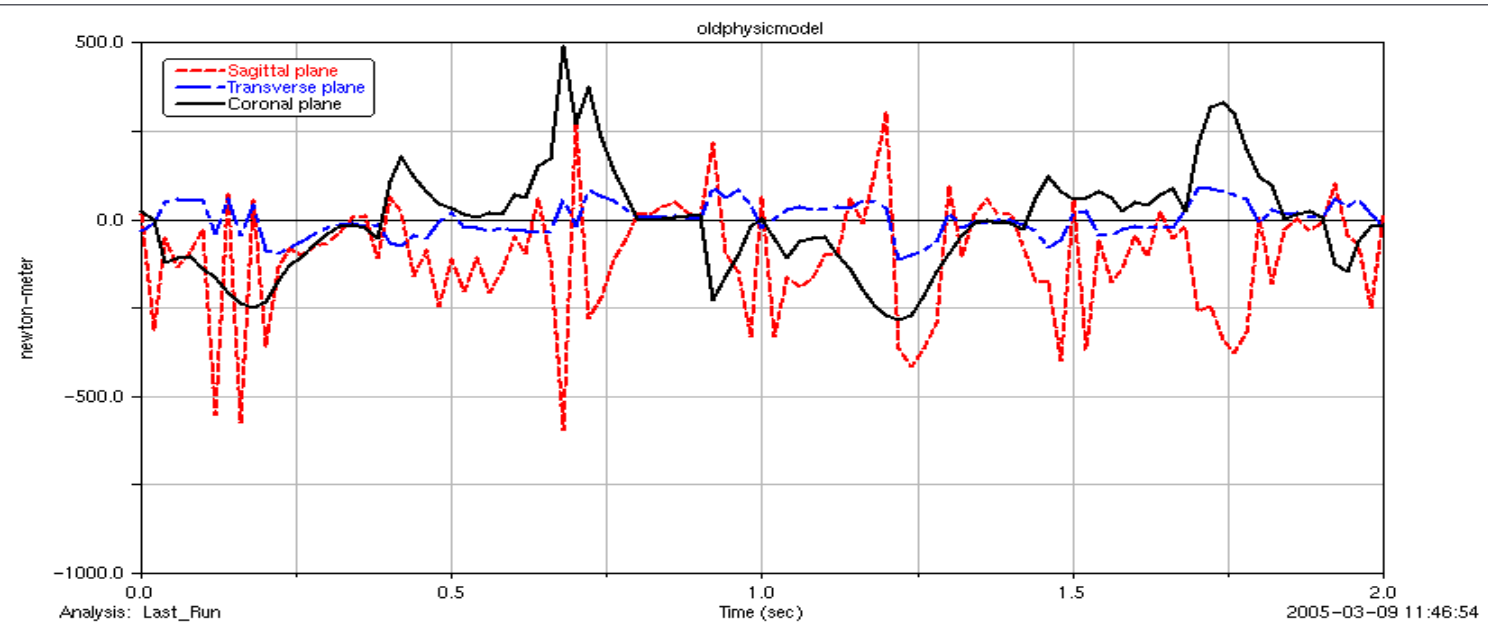

Figure (4 - 16) Control torque in PGO walking

Table (4 - 2): Control Torque

\begin{tabular}{|c|c|c|c|}
\hline Plane & Maximum $(\mathrm{Nm})$ & Minimum $(\mathrm{Nm})$ & Average $(\mathrm{Nm})$ \\
\hline \hline Sagittal & 307.4 & -595.2 & -105.7 \\
\hline Coronal & 489 & -283 & -3.1 \\
\hline Transverse & 90.8 & -114 & 0.7 \\
\hline
\end{tabular}

\subsection{Discussion}

From the simulation there are some problems need to be discussed in detail.

1. Only one leg moving at a time. This is determined in joint motion function. The real PGO walking situation is like that. Maybe sometimes both leg move to adjust the position of legs, but it is restricted in structure. When there is no negative angle for hip 
joint, then hips can not extend. Without extension of the trailing leg, it will not result in a normal continuous smooth biped walking.

\section{To move forward, the patient must lean forward, and the step length depends on the}

leaning angle. This results from only one leg moving at a time. Because stance leg does not move, nor flex or extend, the swing leg can not touch the ground, except in the original position. Just like the normal human stands straight with one leg and moving the other leg, he can not walk forward. When leaning forward, the upright height between hip and ground is less than the length of stance leg, then the swing leg can touch another place except the original. The upright height depends on the leaning angle as does the step length.

\section{Close to normal joint angles of hip and knee did not bring in close to normal gait.}

This is also the result of one leg moving at a time. Because of that, even though the joints move with exact angle, it does not happen at the time it should like normal walking. In normal walking the swing leg moves in a stride as shown in Figure (4 - 17). Here both legs' differences are taken into account, because two legs move at the same time. The stride length is $1.0429 \mathrm{~m}$, the hip angle for toe off is $-29.6^{\circ}$, the hip angle for heel strike is $29^{\circ}$, the total angle is $54.6^{\circ}$. However, in PGO walking the leg just moves in step as shown in Figure (4 - 18). The step length is $0.3259 \mathrm{~m}$, the hip angle for toe off is $1.35^{\circ}$, the hip angle for heel strike is $18.84^{\circ}$, the total angle is $17.5^{\circ}$. The ratio between angles $(54.6 / 17.5=3.12)$ is almost the same as that of step length $(1.0429 /$ $0.3259=3.2)$ 




Figure (4 - 17) Relation of hip and stride in normal walking

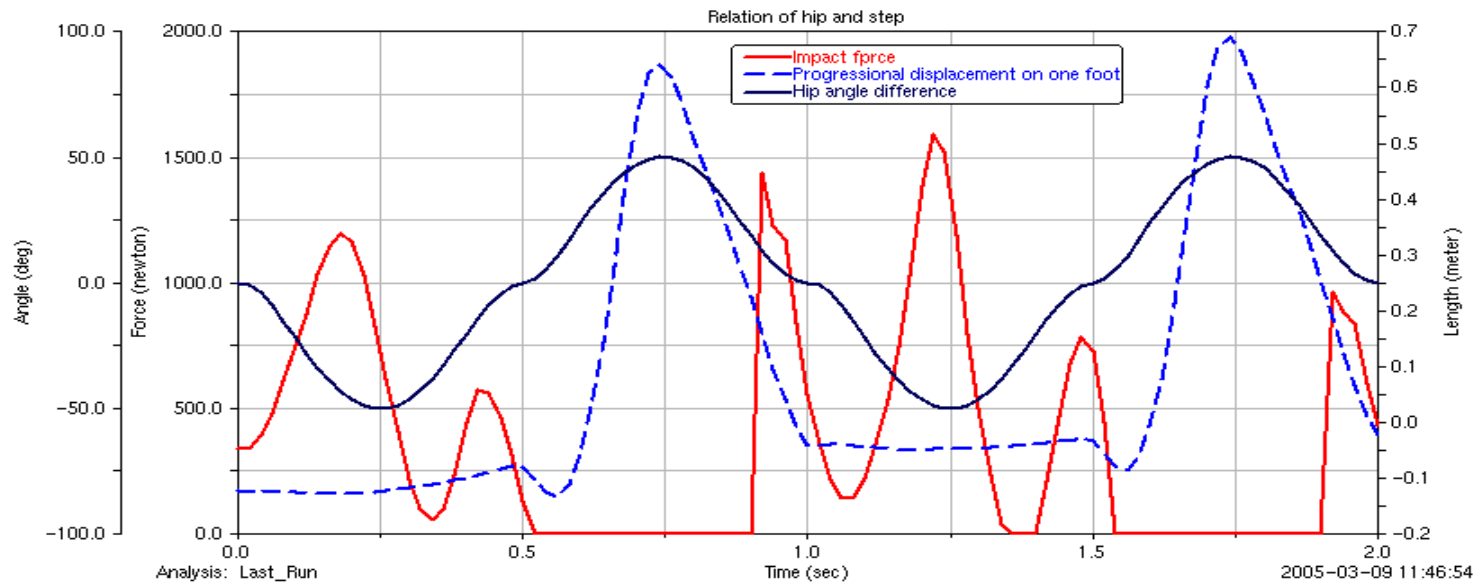

Figure (4 - 18) Relation of hip and step in PGO walking

4. Short step length. It can be seen from above, the step length is $0.3259 \mathrm{~m}$, and it depends on leaning angle. From Figure (4 - 19) at the heel strike the step length is $0.282 \mathrm{~m}$. From simulation it can also be observed that even though the swing leg touches the ground, the mechanism is still moving. This brings the leg backward to 
make the short step even smaller. The effective step length is $0.06 \mathrm{~m}$. It should have brought the trailing body forward, but trailing leg can not lift up to make clearance, the big resistance force under the foot makes it harder to move trailing body forward than to bring one leg back because of weight. From the video (PGO4, BMES, 1994) recorded in the test, this is not true. After the heel strike, no power acts on that foot. The power is switched to the trailing leg, and try to bring the trailing leg to the front of that stance leg. But the trailing leg must first complete the remaining cycle (from heel strike to the next starting point) then start the new cycle. Completing the remaining cycle means the trailing leg extends and has no clearance. That makes trailing leg hard to move forward, and that is why the user twisted his body and readjusted his upper body's position. He was just making clearance to move the trailing leg.

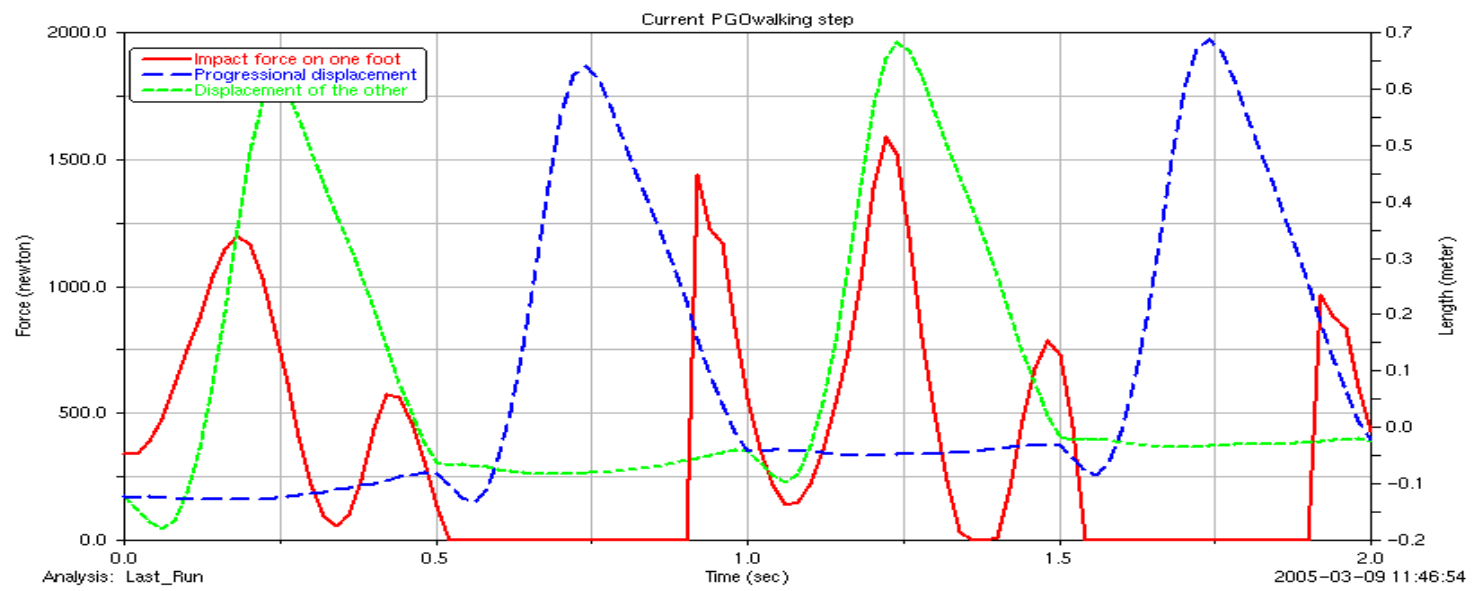

Figure (4 - 19) Step length of PGO walking

5. No clearance or big friction. The clearance here is after toe off clearance for trailing leg. From Figure (4 - 9) It can be seen that there are forces under both feet from heel strike through the trailing leg reaching the same position as forward leg. That means there is no clearance for the trailing leg's movement. The trailing leg has no pre-swing 
and initial swing. They are all counted into double support. And from Figure (4 - 20) a big friction can be seen for both feet, if the same period from 0.9 to 1 second is examined. The blue solid line is for forward leg, red dash line is for trailing leg at that moment. A big forward (positive) friction after heel strike can be seen, that means moving backward, and a small backward (negative) friction on the trailing foot. Because of big force on one leg and small force on the trailing body, the backward sliding of the leg can only be seen. Whatever from simulation or test, the working condition changed a little bit in test, this clearance problem is there. This no clearance results from structure shortage, no power to change the clearance for the trailing leg at that moment. Even though the swing leg has a big movement from stance to heel strike, it has no help to increase the after heel strike clearance.

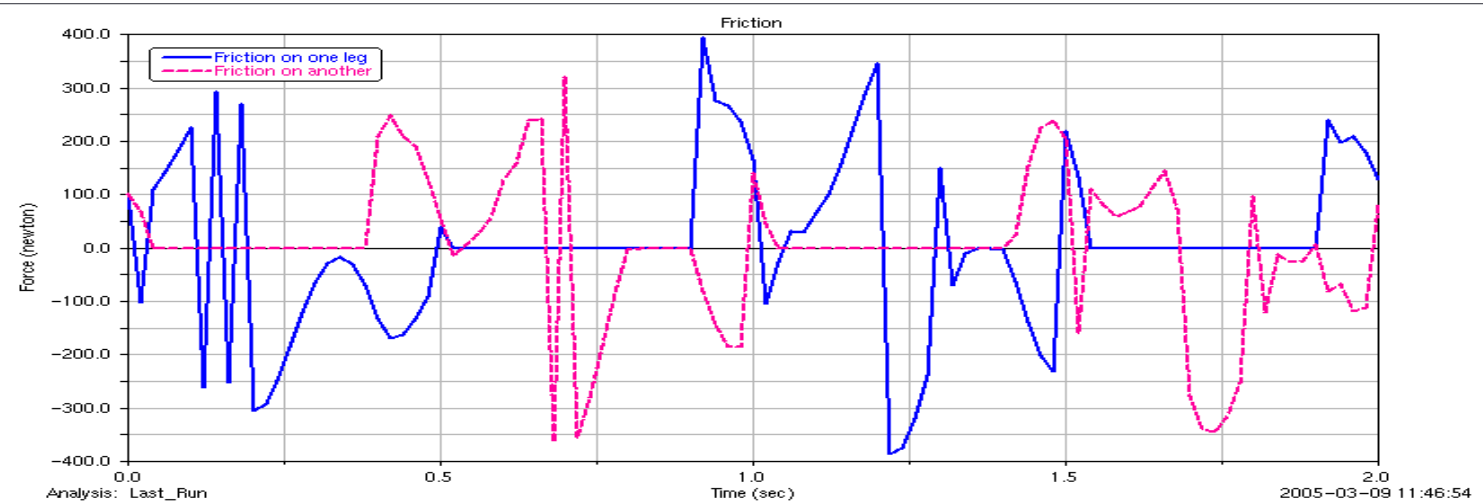

Figure (4 - 20) Friction

6. Mechanical reason for ineffective walking. From Figure (4 - 18) the effective step is only $0.06 \mathrm{~m}$. What is the mechanical reason? From Newton's second law $\mathrm{F}=\mathrm{ma}$, the walking depends on the friction force on stance foot. Only stance foot touches the ground. From the tape observations, the PGO stopped working on the swing foot after it touched the ground, then switched to move another leg. That means it does not help 
with friction force on the stance foot. Then the position of COM can not be changed, because the friction and motion on the stance leg are the power to reposition the COM. For example, if the friction coefficient on the stance foot is assumed to be zero, then any motion on swing leg can not change the position of COM. Therefore, motion on stance leg is necessary. That is why the user used his hands to pull his body forward was observed. Even though the PGO continued to work after the heel strike, the total working time for one leg is $50 \%$ of gait cycle. For the most stance phase (50\% of gait cycle), no force works on it. It is shown in Figure (4 - 21). The total stance phase is $60 \%$ of gait cycle, while $83 \%$ of stance phase has no force. This is the deepest mechanical reason of ineffective PGO walking.
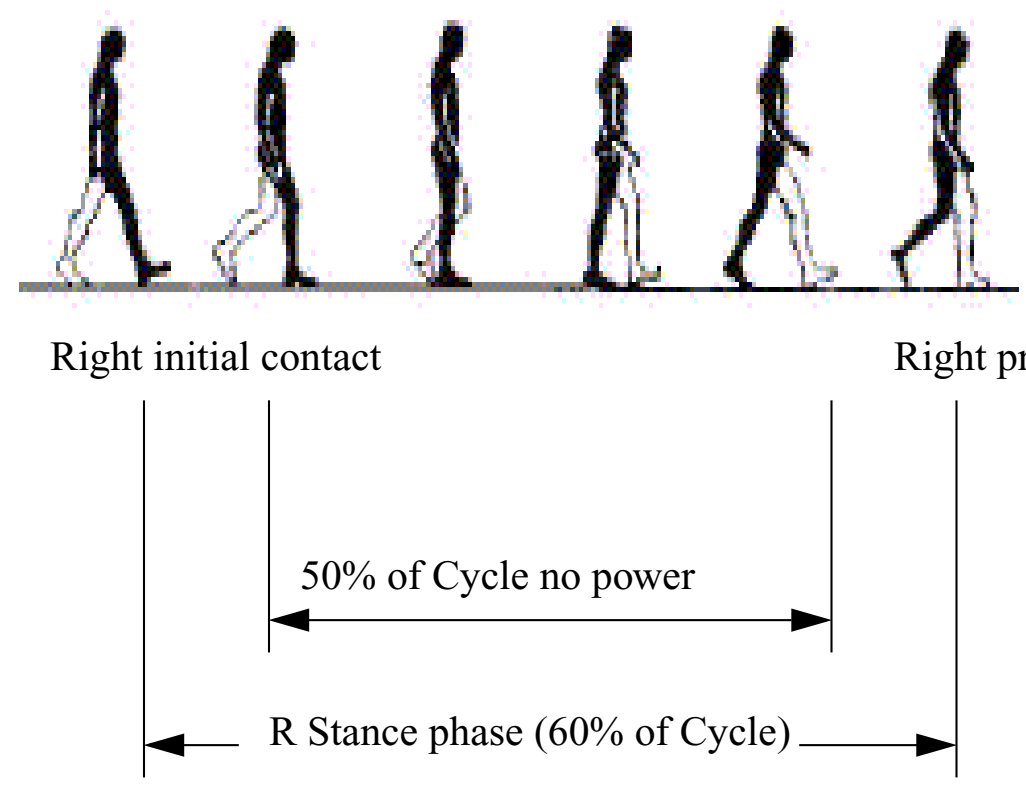

Figure (4 - 21) Stance phase

7. Higher power consumption. From Figure (4 - 15), the average power for one leg is $185 \mathrm{~W}$. In normal walking the simulated average power for one leg is $40 \mathrm{~W}$. When the 
leg swings, there is no friction force on it most of time. But the swing phase is $36 \%$ of gait cycle. From Figure (4 - 20) during 1.5 to $2.0 \mathrm{~s}$ time period, the solid line shows a large force peak on both ends. That happened on double support. The Most power was consumed for these two peaks.

\subsection{Summary}

The direct cause of poor function of current PGO is one leg moving at a time. The mechanical reason is no power working on most of stance phase to move COM forward. 


\section{PGO MECHANISM INVESTIGATION}

As discussed in the last chapter, the real cause of poor function of the current PGO is only one leg moving at a time and no power on the stance phase. Simply adding power on both legs fails to solve the problem. Because it is structurally not allowed negative angle of hip joint, then both legs can not move at the same time. Is it possible to change the initial installation position of both legs to get negative angle for it? Is it possible to merge standing and walking into the device? The initial position is standing. From standing to walking is just walking in a step, but a steady walking is walking in a stride. They are different types of motions. The device must merge these actions. All these can be answered by simulation. However, the best way to do these is to explore all possible solutions to obtain an optimized or trade-off solution.

\subsection{PGO Mechanism Model}

The PGO mechanism model is based on normal walking. The normal gait is tried to be replicated for the patient through the mechanism model. Therefore the main focus is on motions and power consumption. The structure here just serves for these purpose. The real design and how to achieve these motions will be discussed in next chapter. The mechanism model is shown in Figure $(5-1)$. The main features of this model are: 


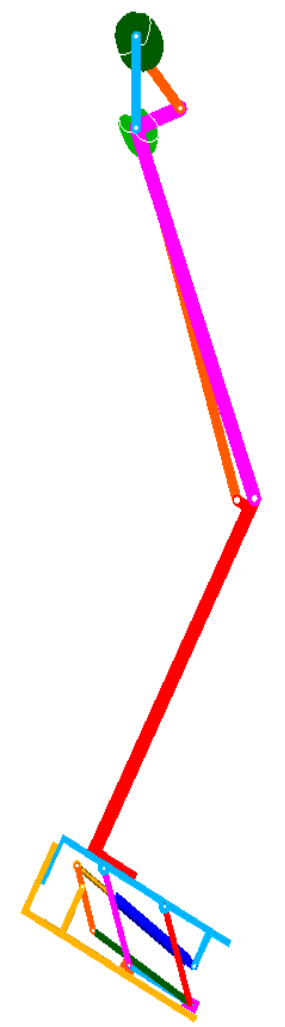

Figure (5 - 1) PGO mechanism model

- Both legs move at the same time.

- The system is of two degrees of freedom for each leg.

- Both hip joint and knee joint functions are approximated by cam-modulated slider crank mechanism and both are coupled. Both joints can exactly replicate the functions of normal walking.

- A foot release mechanism is used to replace ankle joint. The lower plate can retract in the swinging phase and extend to its original length in the stance phase. This motion is different from the ankle motion, but it can help to increase foot/ground clearance.

- Knee or foot release can be locked to show different functions. 


\subsection{Human in PGO Mechanism Model}

To fully understand the function of the PGO mechanism model and without making physical model, we use the same method as that in chapter 3 to simulate human walking in PGO mechanism model. The human in PGO mechanism model is shown in Figure (5 - 2).

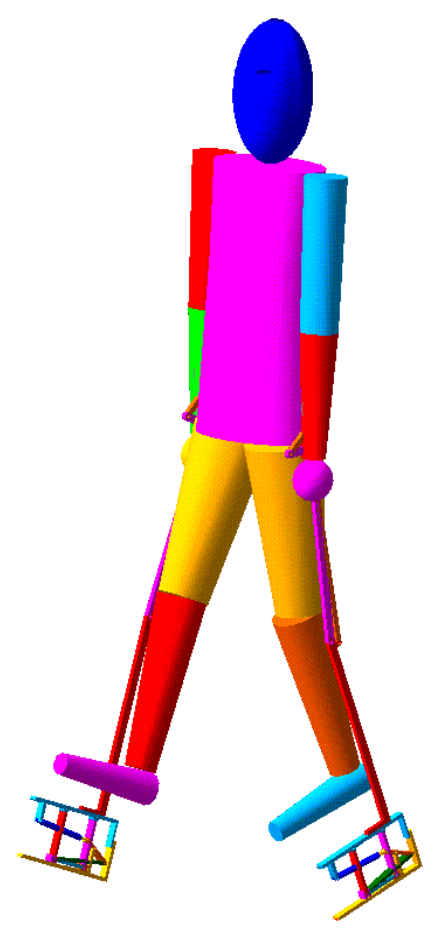

Figure (5 - 2) Human in PGO mechanism model

The PGO size is not the exact size of human model. The Hip joint of PGO is in line with the hip joint of human model. Both hip joint and knee joint of the human model are deactivated. The whole weight of a human is supported by PGO.

\subsection{Joint Motion Function}

The motion function of the hip and knee are exactly the same as those in the human model. All these motions are input first to simulate the PGO to obtain the correct cam pro- 
files, then use the correct cam profiles to make cam-follower joints. By providing a steady continuous rotation function, both hip and knee joint motion functions can be exactly modeled. A continuous rotation function means both legs move at the same time. This is different from current PGO physical model, where only one leg moves at a time.

Now motion function for foot release mechanism needs to be created. This motion must be continuous for the sake of ADAMS, thus Fourier series is still used here as shown in Figure (5 - 3).

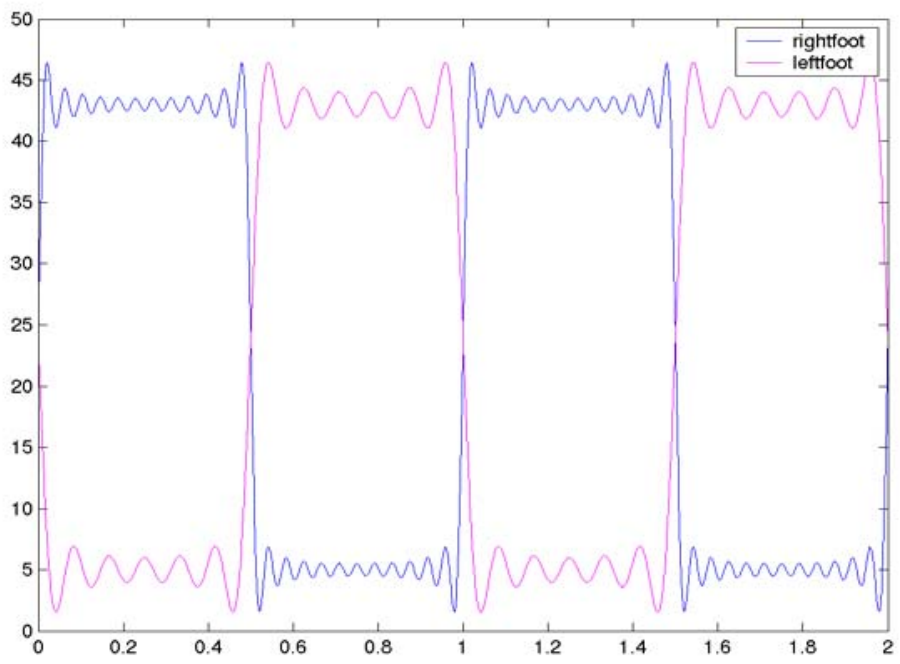

Figure (5 - 3) Fourier series of foot release mechanism

\subsection{Simulation}

To explore and compare functions of different type of PGO, both knee and ankle can move, or knees are locked but ankles can move, or ankles are locked but knees can move. They are called Foot-Knee Release PGO, Foot Release PGO and Knee Release PGO, respectively. In order to choose a more reasonable PGO, simulations on these three types 
of PGO, foot-knee release PGO, foot release PGO and knee release PGO are executed. They are shown in Figure (5 - 4) and Figure (5 - 5). The simulation of knee release PGO walking is not shown, it looks like foot knee release PGO walking.

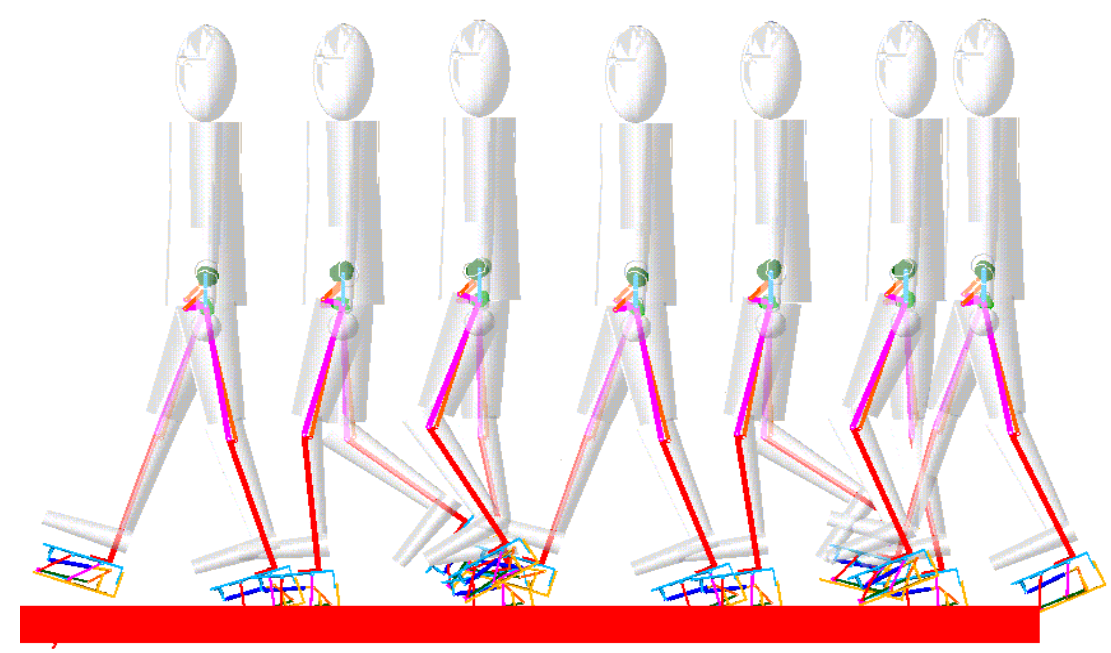

Figure (5 - 4) Simulation of foot knee release PGO walking

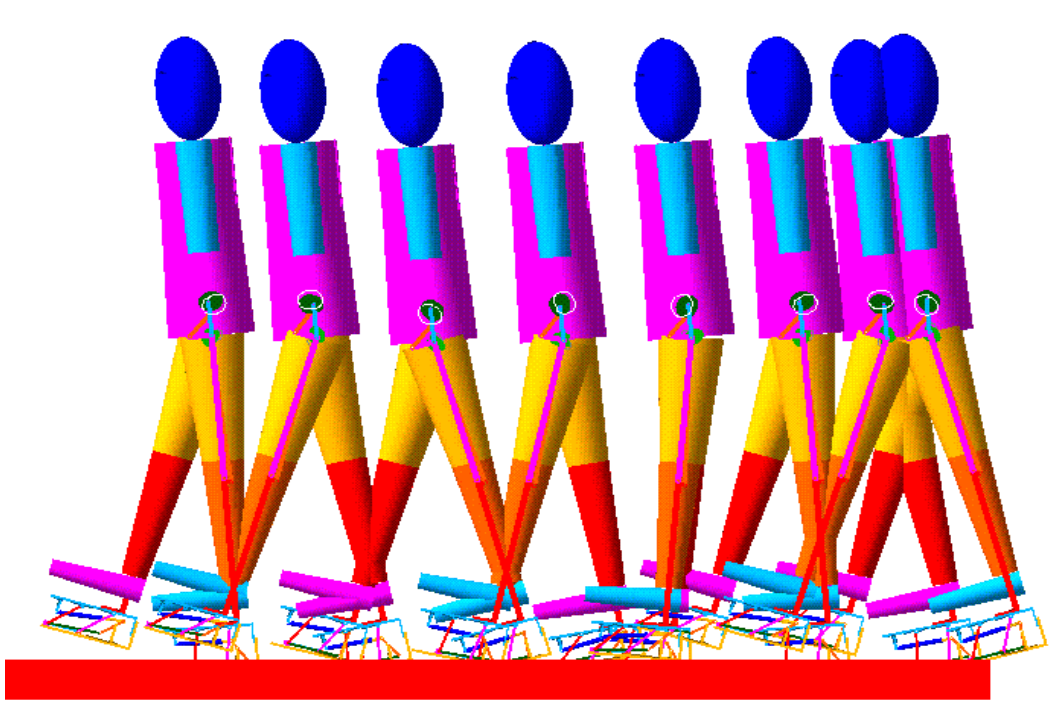

Figure (5 - 5) Simulation of foot release PGO walking 


\subsection{Analysis of simulation results}

\subsubsection{Ground reaction force}

The ground reaction impact forces are shown in Figure (5 - 6). On the left are impact forces. Corresponding frictions are on the right. Put together, it is easier to see the difference. The impact forces of PGO mechanism model, normal walking and current PGO are shown in Figure (5 - 7). The detailed data is shown in Table 5 - 1 on page 83. It seams that average force is half of the weight. Only current PGO walking has three peaks, highest maximum force and longest stance phase.

Table(5 - 1): Maximum force and Stance phase of PGO Mechanism Walking

\begin{tabular}{c|c|c|c}
\hline & $\begin{array}{c}\text { Maximum } \\
\text { Force }(\mathrm{N})\end{array}$ & $\begin{array}{c}\text { Average } \\
(\mathrm{N})\end{array}$ & $\begin{array}{c}\text { Stance Phase } \\
(\% \text { of cycle })\end{array}$ \\
\hline Normal walking & 808.4 & 353.8 & 62 \\
\hline Current PGO walking & 1672 & 343.4 & 66 \\
\hline Foot knee release walking & 1197.3 & 389.4 & 52 \\
\hline Knee release walking & 1278 & 398.9 & 54 \\
\hline Foot release walking & 988.9 & 390.2 & 60 \\
\hline
\end{tabular}

The reason for higher impact forces and short stance phase in PGO mechanism results mainly from:

- $\quad$ PGO adds weight to the whole model

- Legs are longer than in human model, therefore the position of COM is higher, and mass distribution is changed

- Lower legs are not the same length as upper legs. 


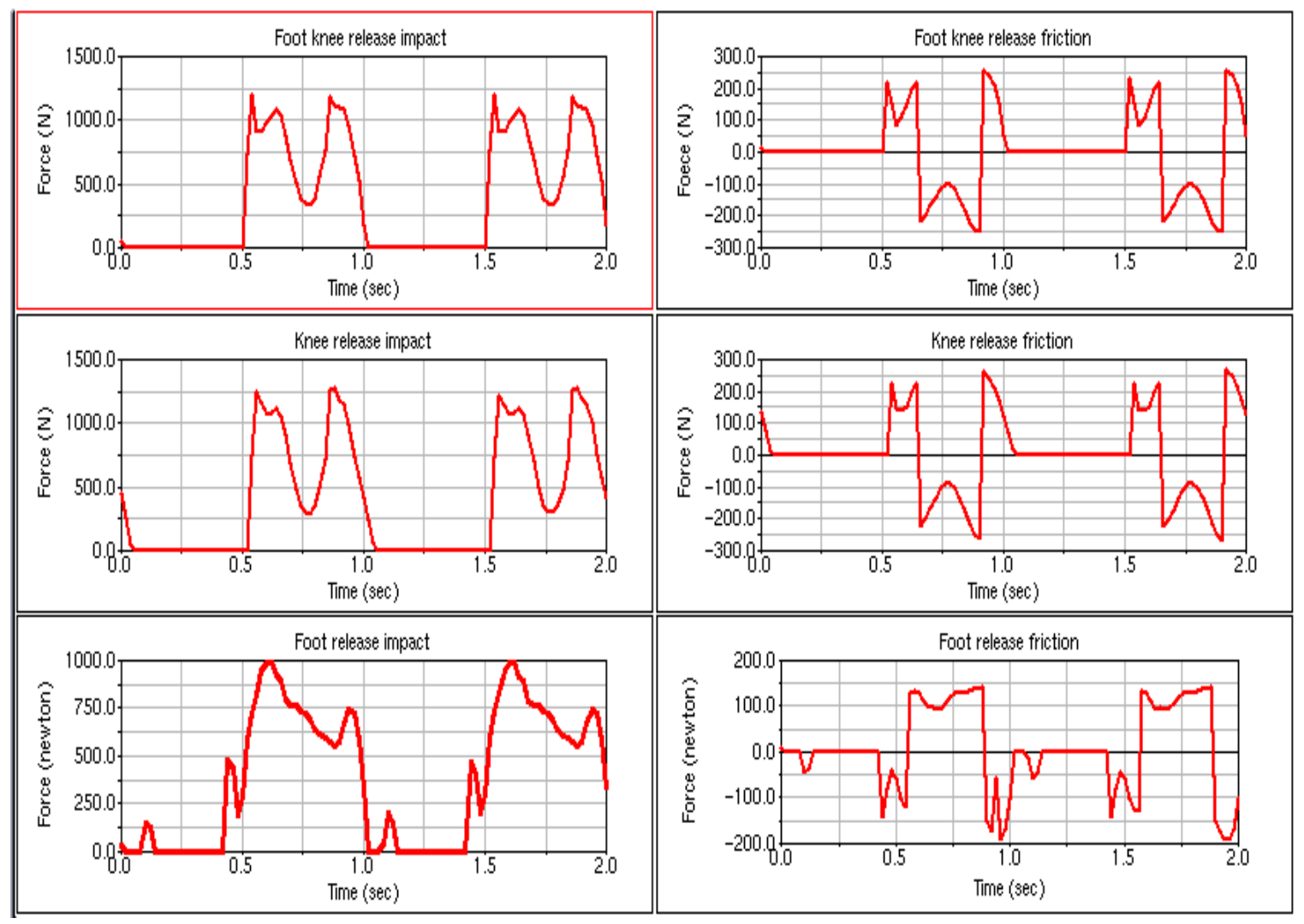

Figure (5 - 6) Ground reaction force on PGO mechanism model

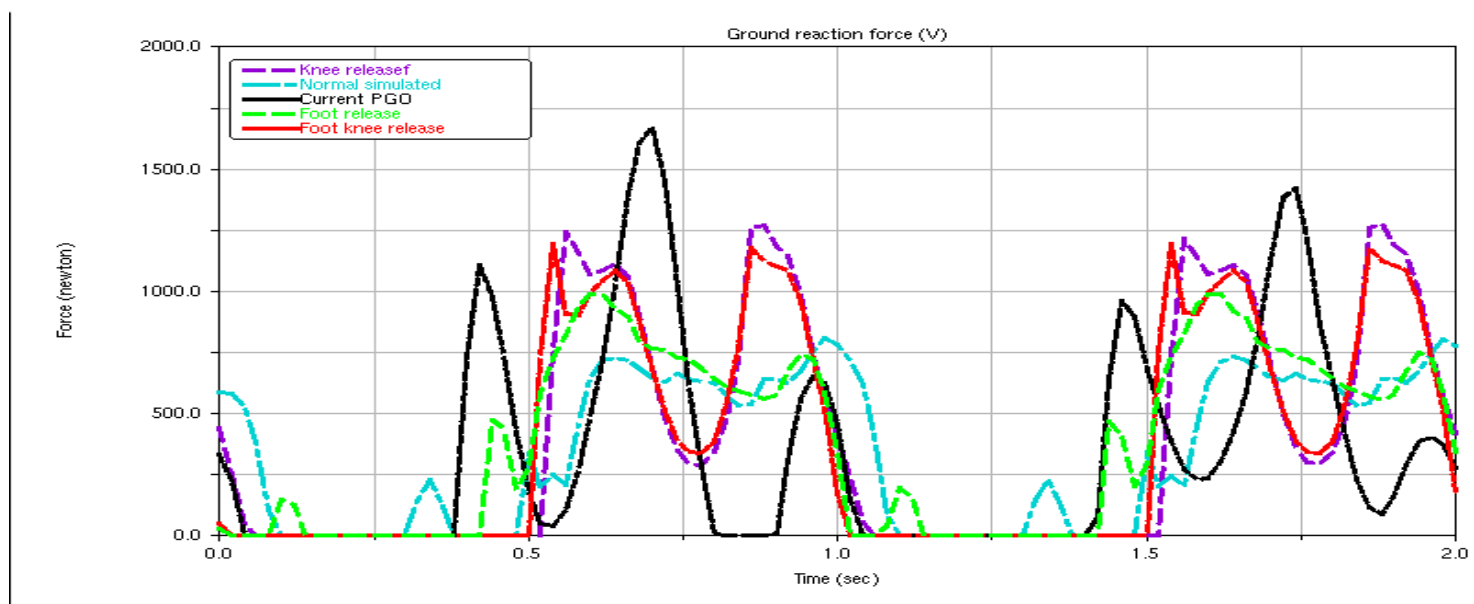

Figure (5 - 7) Impact force comparison 
Progressional force are put together in Figure (5 - 8), in order to demonstrate the difference of curve forms. Their values are not compared. The friction curve of foot release is different. Before toe off there is no push forward force like in other models. That is why the walking efficient of foot release is lower than other models as seen in step comparison.

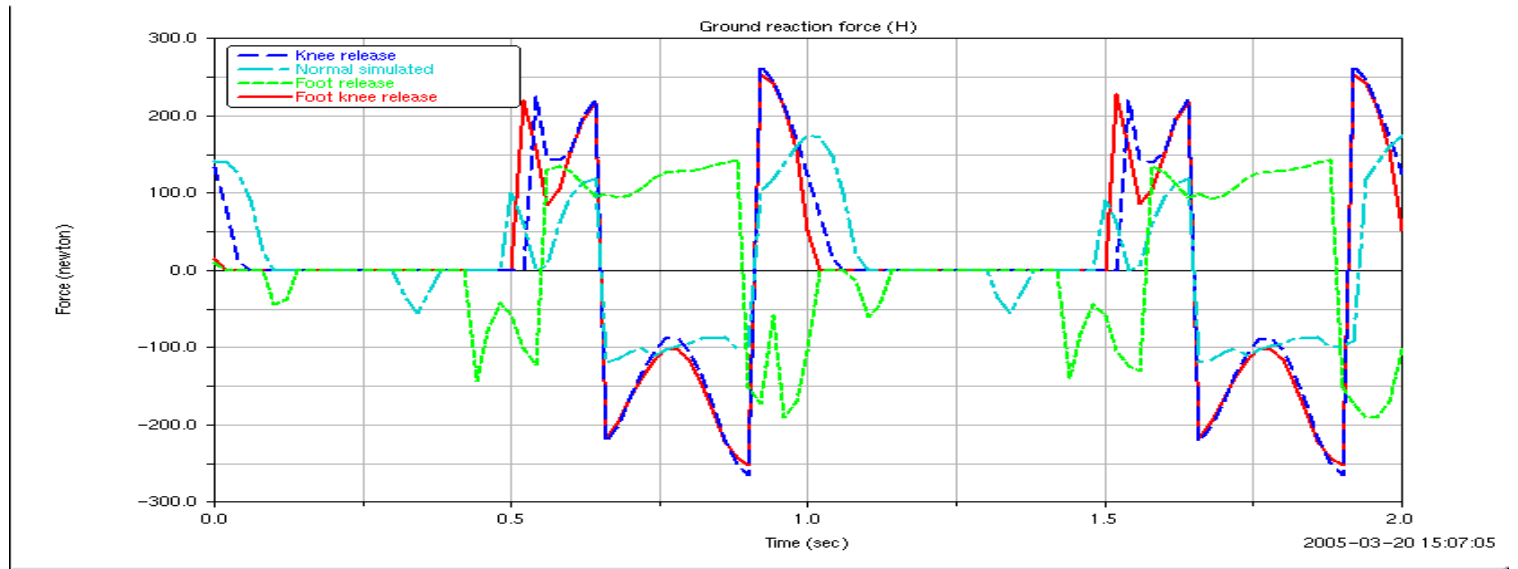

Figure (5 - 8) Friction comparison

\subsubsection{Sliding and Penetration}

Slidings are shown in Figure (5 - 9). Sliding has a big affect on step or stride length. The sliding distances are measured from heel strike to toe off, in the duration of stance phase. They are put in Table $5-2$ on page 85 .

Table(5 - 2): Sliding of PGO mechanism Walking

\begin{tabular}{c|c}
\hline & Sliding $(\mathrm{M})$ \\
\hline Foot knee release & 0.153 \\
\hline Knee release & 0.167 \\
\hline Foot release & -0.075 \\
\hline
\end{tabular}




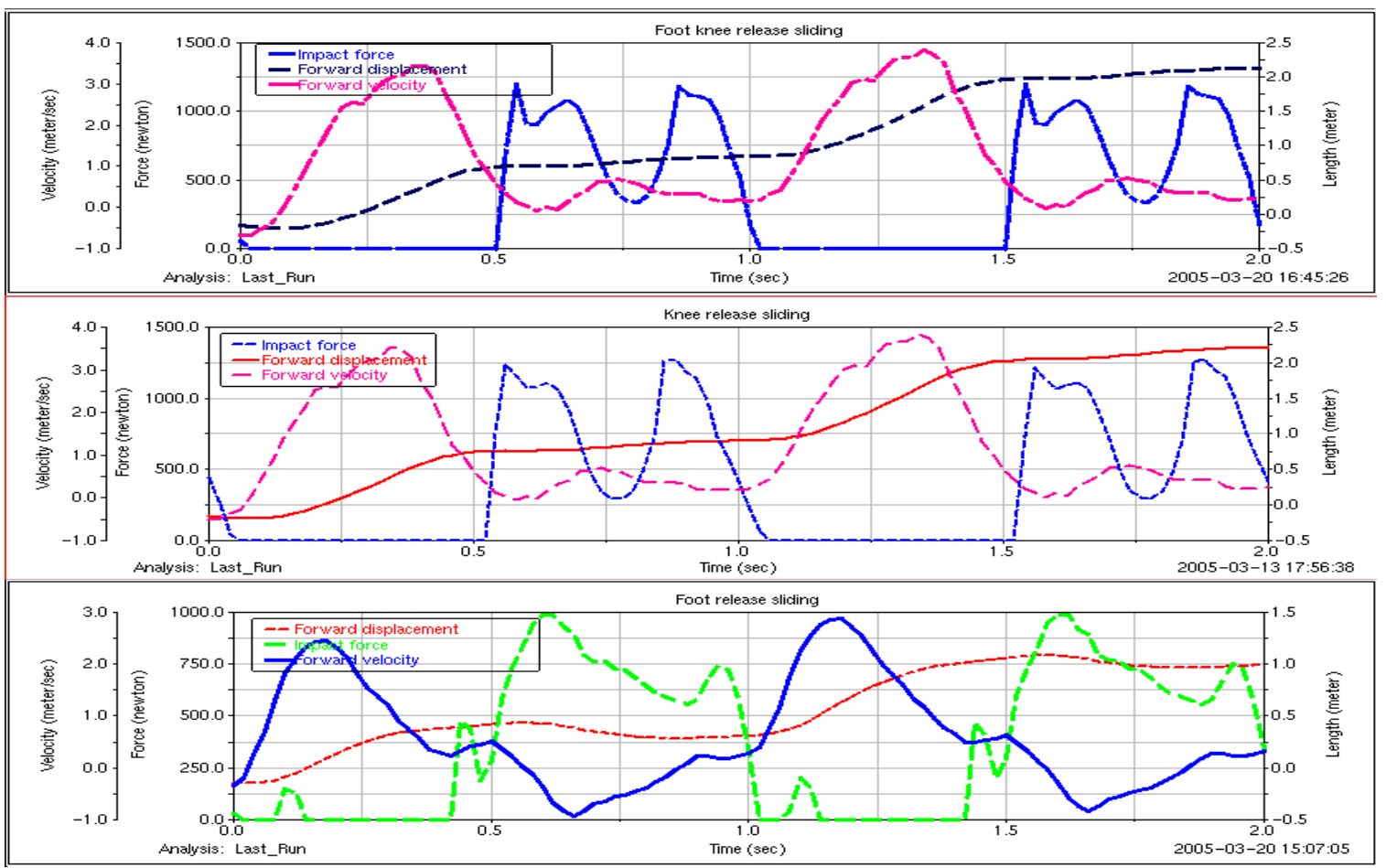

Figure (5 - 9) Sliding of PGO mechanism model

Penetration is shown in Figure (5 - 10). The displacement is for heel and toe on one foot. For the stance leg it is called penetration; for the swing leg it is called clearance. Because of the penetration of the stance leg, the clearance of the swing leg is decreased, it appears as if the zero line moves up to increase penetration and decrease the clearance. Because it depends on the motion you assign, it can not move like a human to intentionally avoid obstacle. 


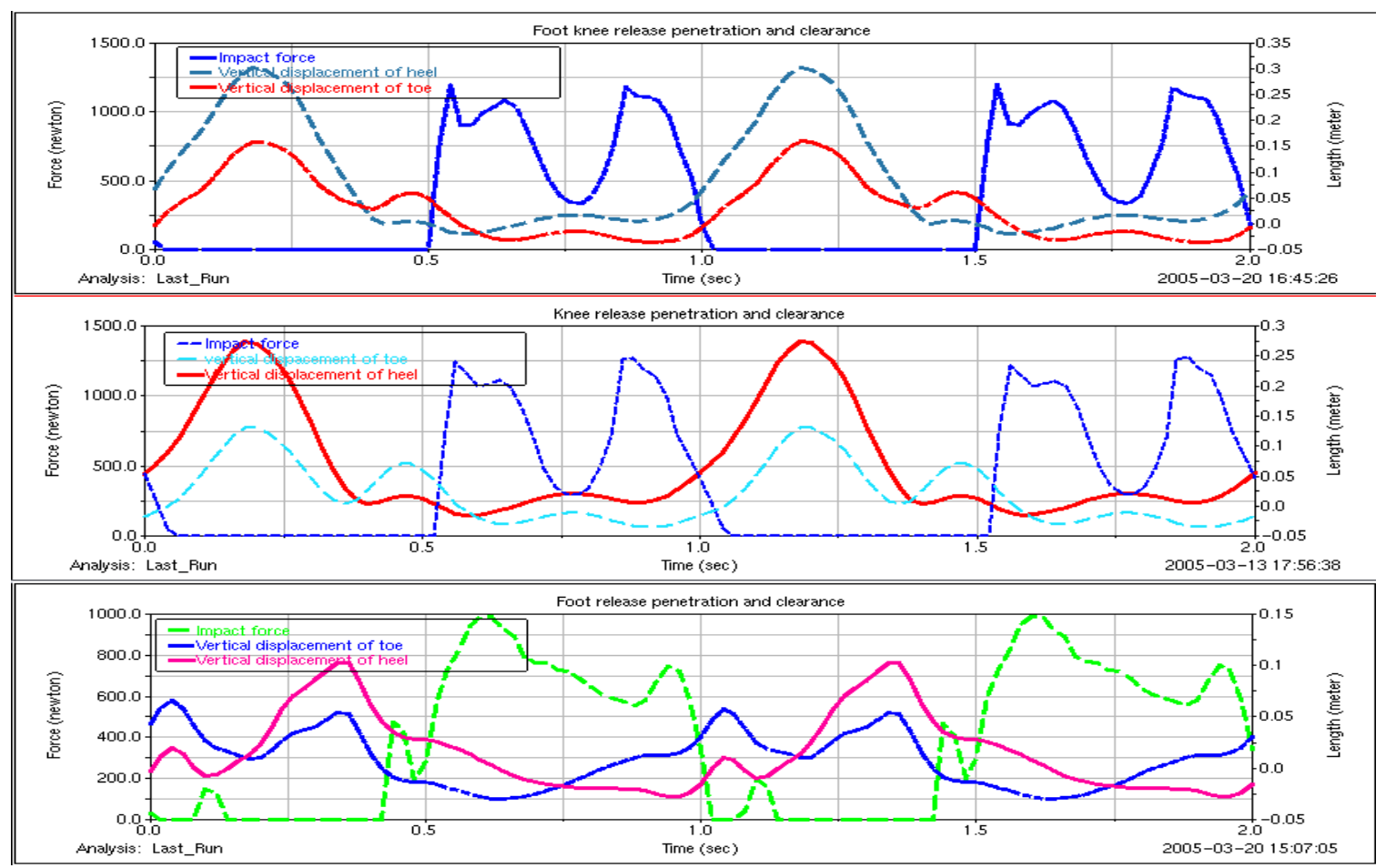

Figure (5 - 10) Penetration of PGO mechanism model

\subsubsection{The Center of Mass}

The COM is shown in Figure (5 - 11). Compared with Normal walking, The COMs of PGO mechanism walking have higher oscillation. From normal walking, we can see the lowest points are in double support. But in PGO mechanism model walking they shift slightly. That suggests after the double support there is a large penetration in the simulation. 


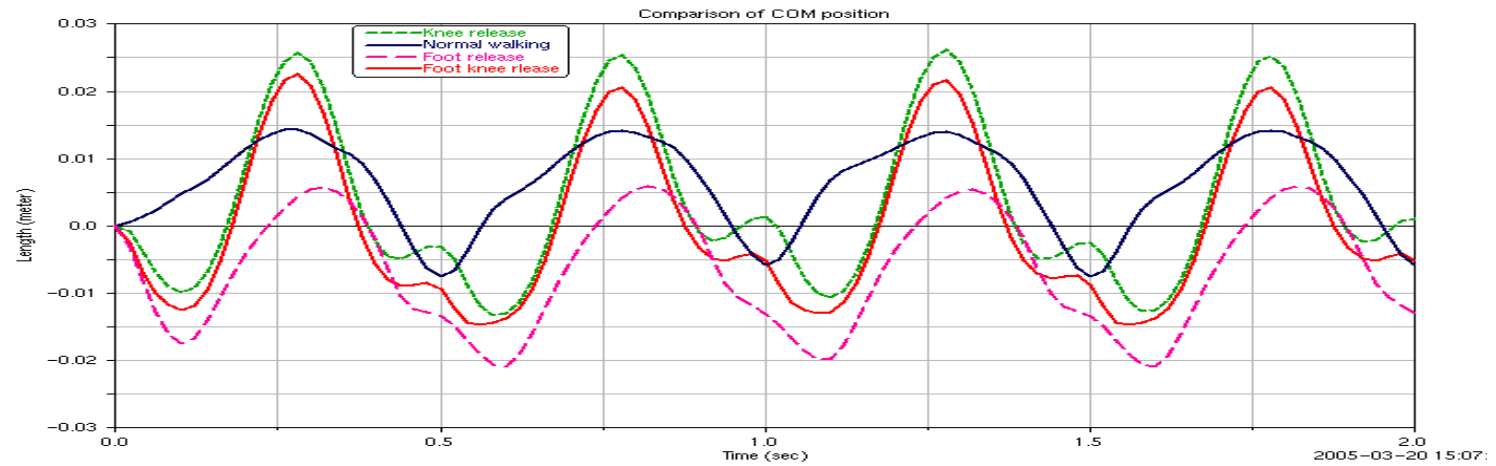

Figure (5 - 11) COM of PGO mechanism model

\subsubsection{Joint Motions}

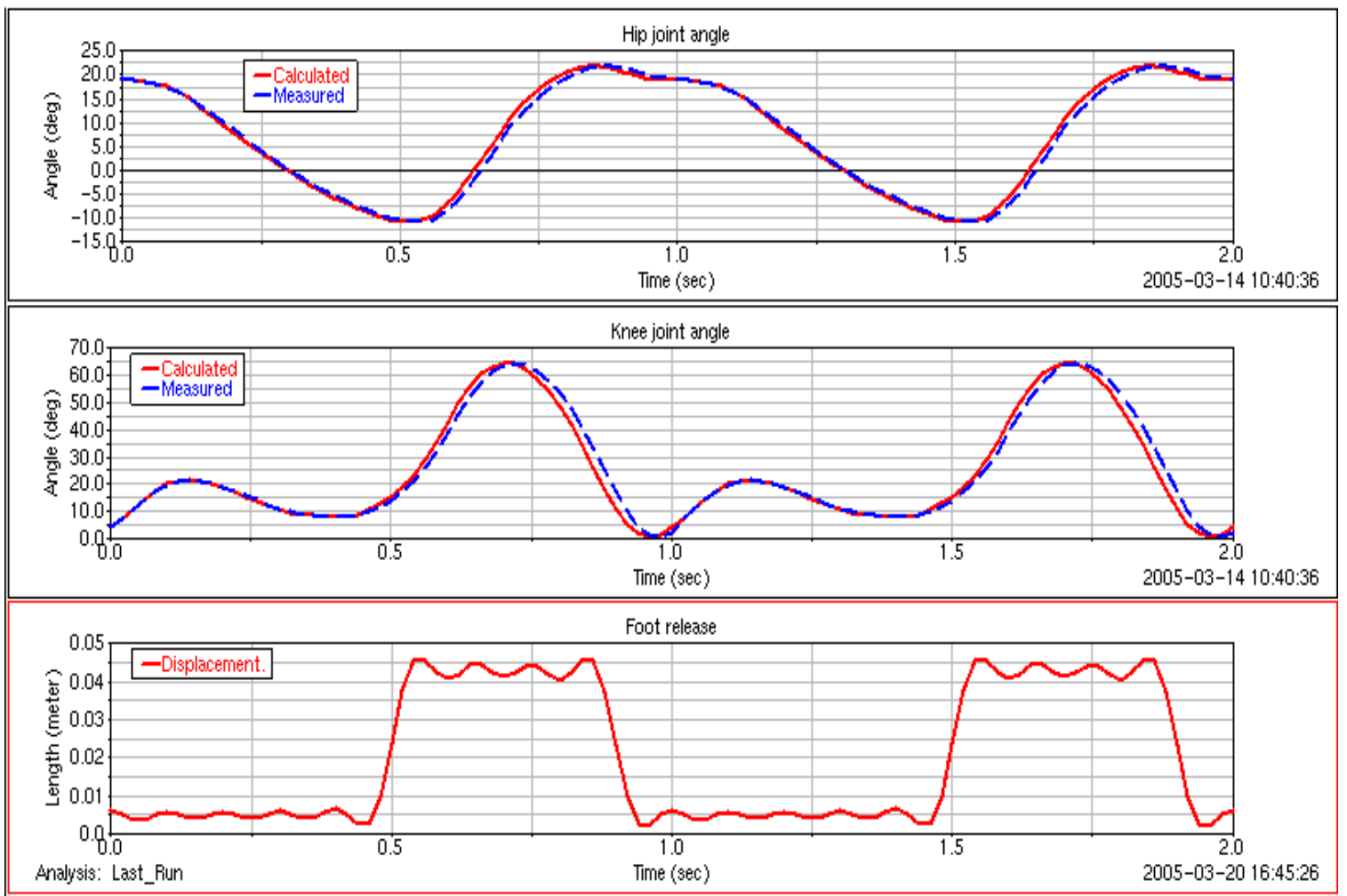

Figure (5 - 12) Joint angles of PGO mechanism model 
Joint motions are our final goal. The normal joint movement can be replicated to achieve normal walking even though it costs more energy than normal walking. Figure (5 - 12) shows that foot knee release and knee release model can exactly replicate hip joint and knee joint motions in sagittal plane. Foot release can replicate hip motion, but not knee joint motion. Foot release in place of ankle joint means that translation replaces revolution. It works only at the moment on toe off and before heel strike to make clearance for swing phase. There is no movement in stance phase. The rip on the curve is from the Fourier series. As discussed in last chapter, if it does not work in stance phase, it does not contribute to move COM. The stance phase for foot release is $60 \%$ of the gait cycle.

\subsubsection{Joint Moment and Power Consumption}

The joint moment and power consumption of PGO mechanism model are shown in Figure (5 - 13), Figure (5 - 14) and Figure (5 - 15). The total power for one leg is shown in Figure (5 - 16). Be reminded that foot release only works at the moment on toe off and before heel strike with no force acting on it. Therefore, it only needs a very small power to overcome the joint friction and self weight of lower plate. From Figure (5 - 13) and Figure (5 - 15) you can see the average power is negative. That is not true for the real case. Here we want to compare the power consumption ignoring the foot release power. The data excerpted from these figures is shown in Table 5 - 3 on page 90 . The average power consumption has no significant difference. 
Table(5 - 3): Torque and Power for Mechanism Model

\begin{tabular}{c|c|c|c|c}
\hline \multirow{2}{*}{} & \multicolumn{4}{|c}{ Moment (Nm) } \\
\cline { 2 - 5 } & \multicolumn{2}{|c}{ Hip } & \multicolumn{2}{c}{ Knee } \\
\cline { 2 - 5 } & Max & Ave & Max & Ave \\
\hline $\begin{array}{c}\text { Foot knee } \\
\text { release }\end{array}$ & 68.7 & 8.4 & 102 & -5.4 \\
\hline $\begin{array}{c}\text { Knee } \\
\text { release }\end{array}$ & 80.45 & 8.4 & 78.7 & -5.6 \\
\hline $\begin{array}{c}\text { Foot } \\
\text { release }\end{array}$ & 77 & 12.9 & 0 & 0 \\
\hline
\end{tabular}

(Continued)

\begin{tabular}{c|c|c|c|c|c|c}
\hline \multirow{2}{*}{} & \multicolumn{5}{c}{ Power (W) } \\
\cline { 2 - 7 } & \multicolumn{2}{|c}{ Hip } & \multicolumn{2}{c}{ Knee } & \multicolumn{2}{c}{ Hip \& Knee } \\
\cline { 2 - 7 } & Max & Ave & Max & Ave & Max & Ave \\
\hline $\begin{array}{c}\text { Foot knee } \\
\text { release }\end{array}$ & 432 & 53 & 642.6 & 34 & 688.6 & 87 \\
\hline $\begin{array}{c}\text { Knee } \\
\text { release }\end{array}$ & 505.5 & 52.8 & 494 & 35 & 648.7 & 88 \\
\hline $\begin{array}{c}\text { Foot } \\
\text { release }\end{array}$ & 485.4 & 81 & 0 & 0 & 485.4 & 81 \\
\hline
\end{tabular}




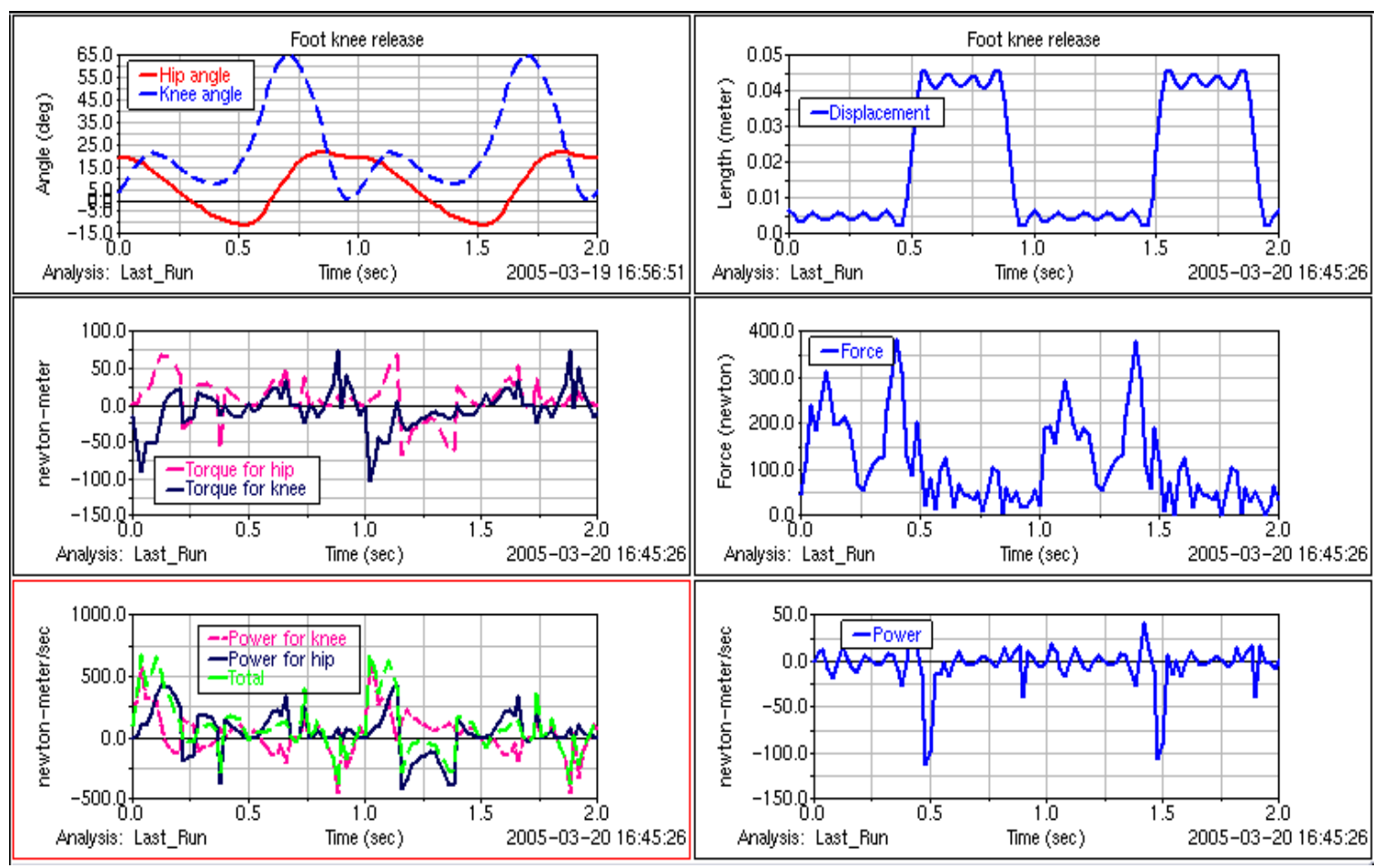

Figure (5 - 13) Moment and power for foot knee release PGO

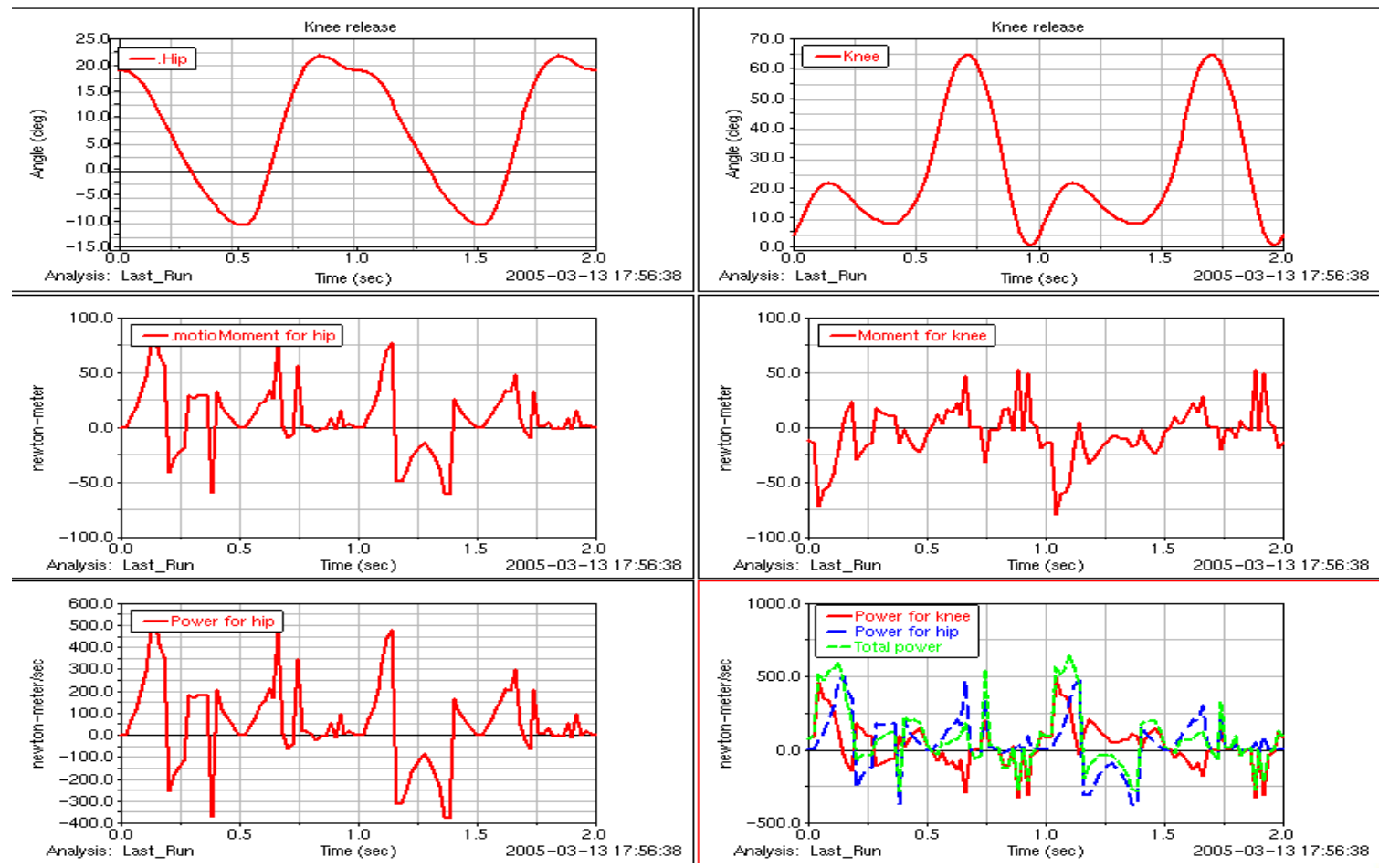

Figure (5 - 14) Moment and power for knee release PGO 


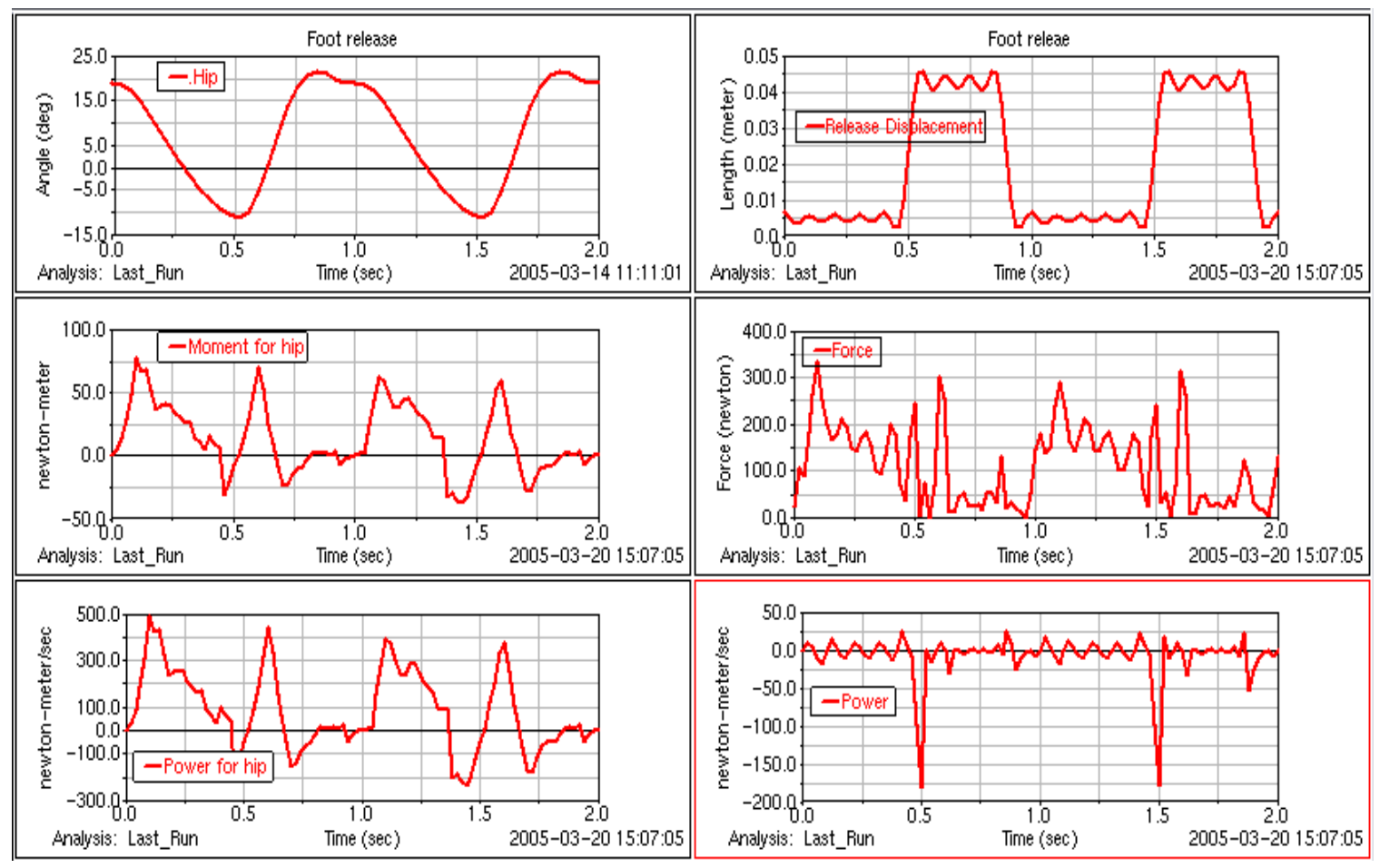

Figure (5 - 15) Moment and power for foot release PGO

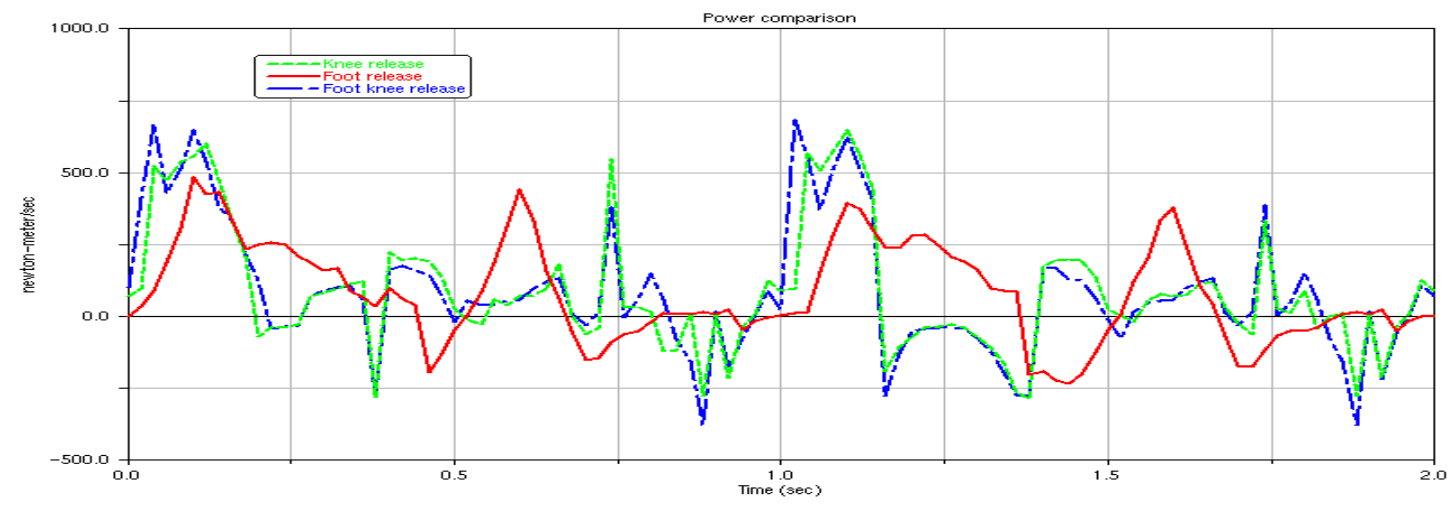

Figure (5 - 16) Power comparison of PGO mechanism model 


\subsubsection{Control Torque}

The torques to keep upper body's balance are shown in Figure (5 - 17) and comparison of these torques is shown in Figure $(5-18)$. The data from these figures is shown in Table 5 - 4 on page 93.

Table(5 - 4): Control Torque of PGO Mechanism Model

\begin{tabular}{c|c|c|c|c|c|c|c|c|c}
\hline & \multicolumn{3}{|c|}{ Maximum (Nm) } & \multicolumn{3}{c|}{ Minimum (Nm) } & \multicolumn{3}{c}{ Average (Nm) } \\
\cline { 2 - 10 } & $\mathrm{F} \& \mathrm{~K}$ & $\mathrm{~K}$ & $\mathrm{~F}$ & $\mathrm{~F} \& \mathrm{~K}$ & $\mathrm{~K}$ & $\mathrm{~F}$ & $\mathrm{~F} \& \mathrm{~K}$ & $\mathrm{~K}$ & $\mathrm{~F}$ \\
\hline $\begin{array}{c}\text { Sagit- } \\
\text { tal }\end{array}$ & 531.8 & 896 & 407 & -376.5 & -1072 & -491 & 46.4 & 39.7 & 63 \\
\hline $\begin{array}{c}\text { Coro- } \\
\text { nal }\end{array}$ & 246.6 & 259 & 199.7 & -218.8 & -245 & -181.5 & 1.04 & -2.4 & 1.8 \\
\hline $\begin{array}{c}\text { Trans- } \\
\text { verse }\end{array}$ & 76.3 & 74 & 74.3 & -86.3 & -69 & -82.7 & -5.9 & -5.4 & -.0 .54 \\
\hline
\end{tabular}




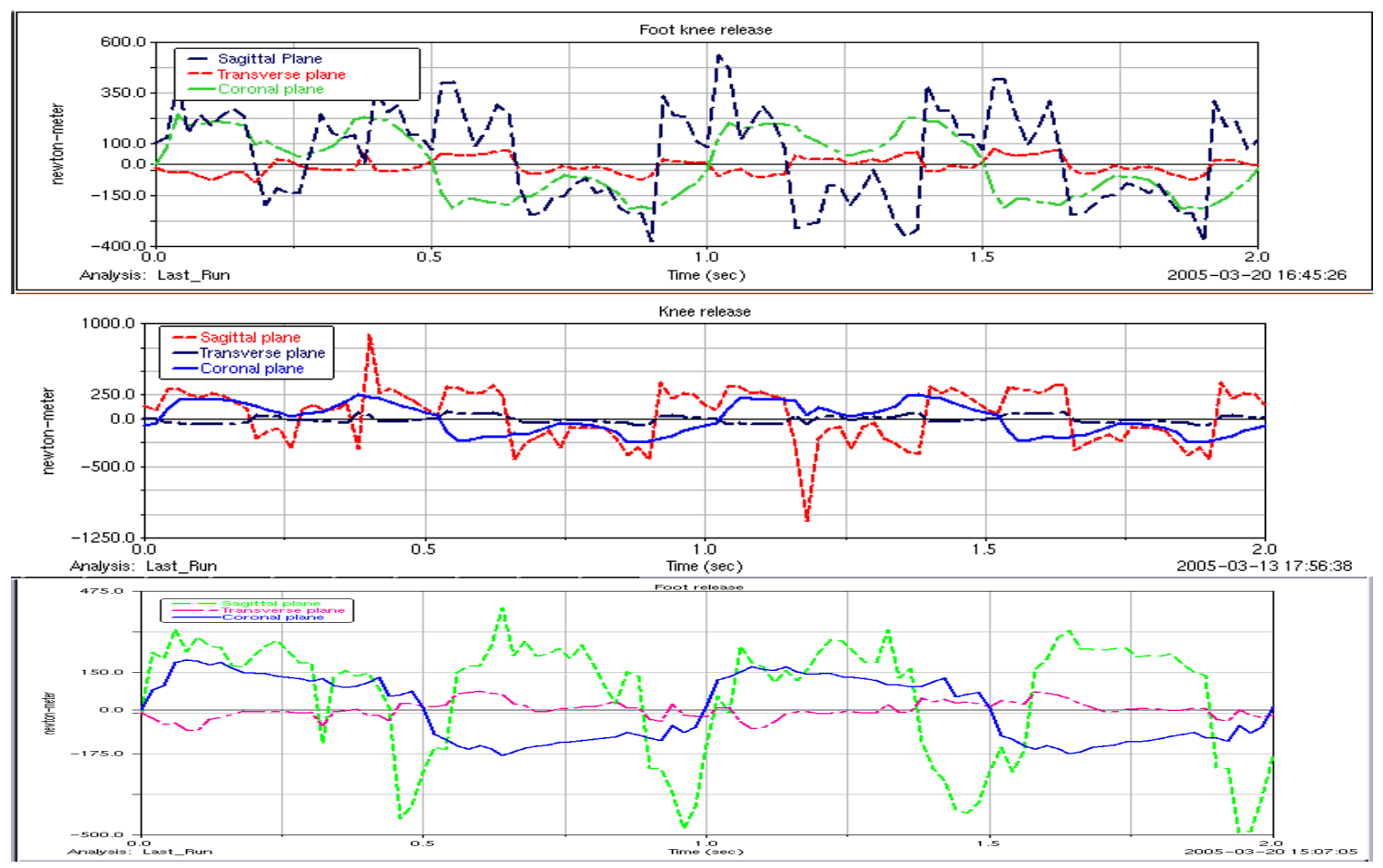

Figure (5 - 17) Control torque of PGO mechanism model

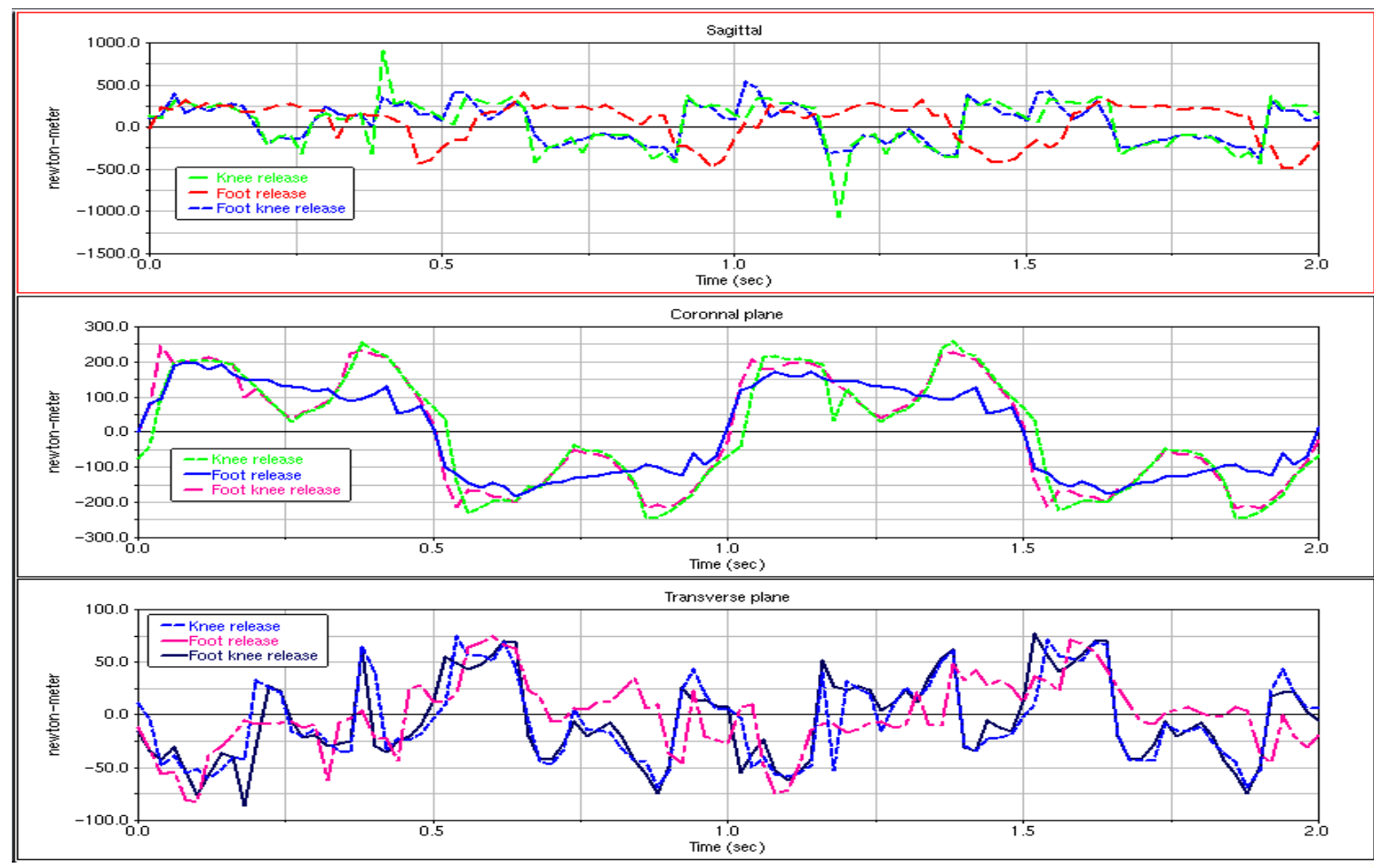

Figure (5 - 18) Comparison of control torque of PGO mechanism model 


\subsection{Discussion}

1. Power Ratio. The step length is measured at the starting point of the ground reaction force, i.e. the heel strike point. The curves for measuring step length of the PGO mechanism model are shown in Figure (5 - 19). The walking distance in two cycles is shown in Figure ( 5 - 20). The data is summed in Table 5 - 5 on page 95. The data demonstrates the sliding indeed has an effect on stride. Because PGO increases the length of legs, the step length is not comparable. The power ratio here is the average power consumption of both legs in unit walking distance. The lower the better. Inarguably, normal walking is the most efficient.

Table(5 - 5): Comparison of Power Ratio

\begin{tabular}{c|c|c|c|c}
\hline & Step $(\mathrm{m})$ & Stride $(\mathrm{m})$ & $\begin{array}{c}\text { Distance in 2 } \\
\text { sec. }(\mathrm{m})\end{array}$ & $\begin{array}{c}\text { Power ratio } \\
(\mathrm{W} / \mathrm{m})\end{array}$ \\
\hline Foot knee release & 0.4945 & 1.1105 & 2.2987 & 75.69 \\
\hline Knee release & 0.5004 & 1.1179 & 2.3783 & 74 \\
\hline Foot release & 0.394 & 0.703 & 1.1364 & 142.56 \\
\hline Current PGO & 0.282 & 0.3259 & 0.10 & 1700 \\
\hline Normal walking & 0.4673 & 1.0429 & 1.7772 & 63.25 \\
\hline
\end{tabular}




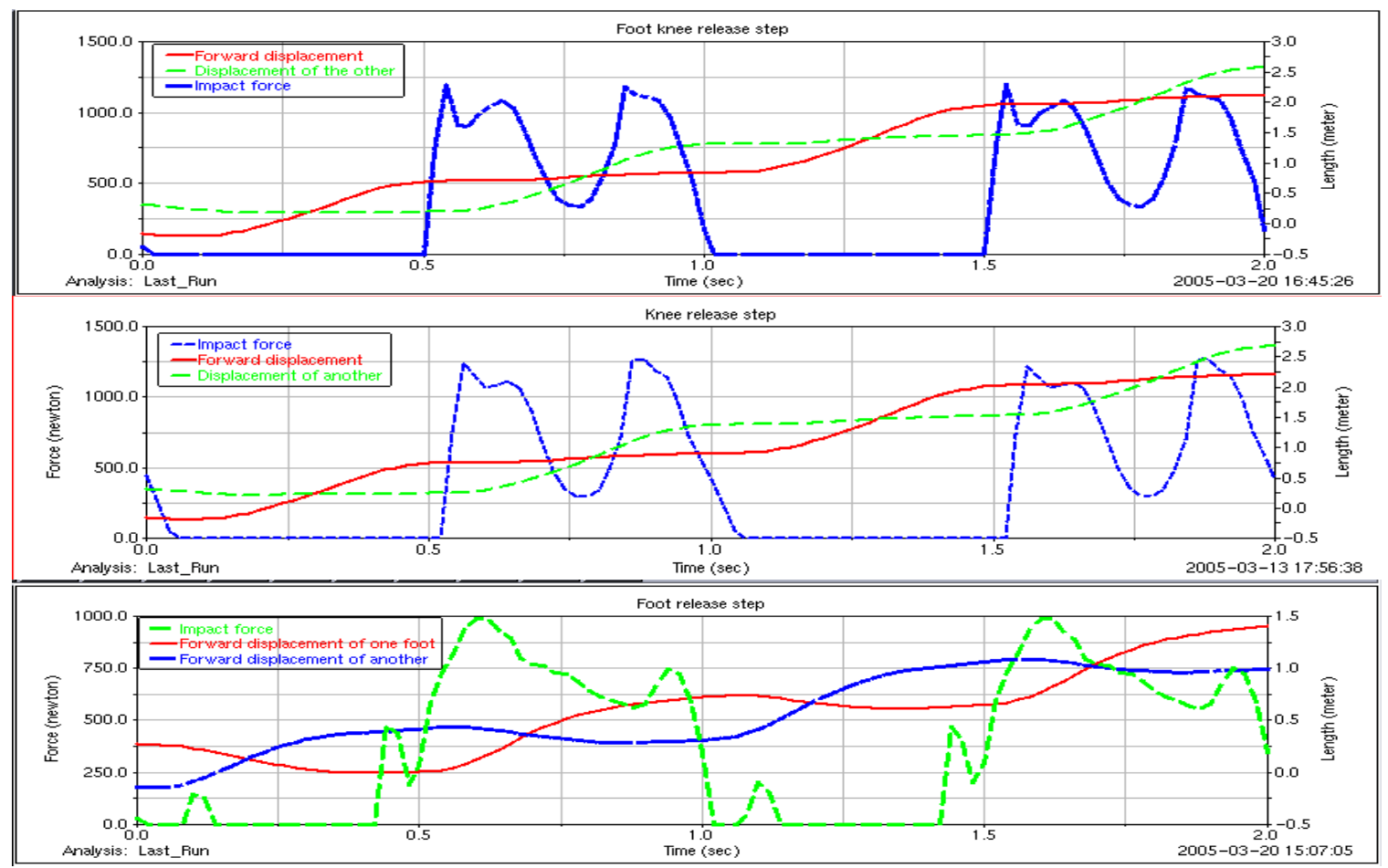

Figure (5 - 19) Step length of PGO mechanism model

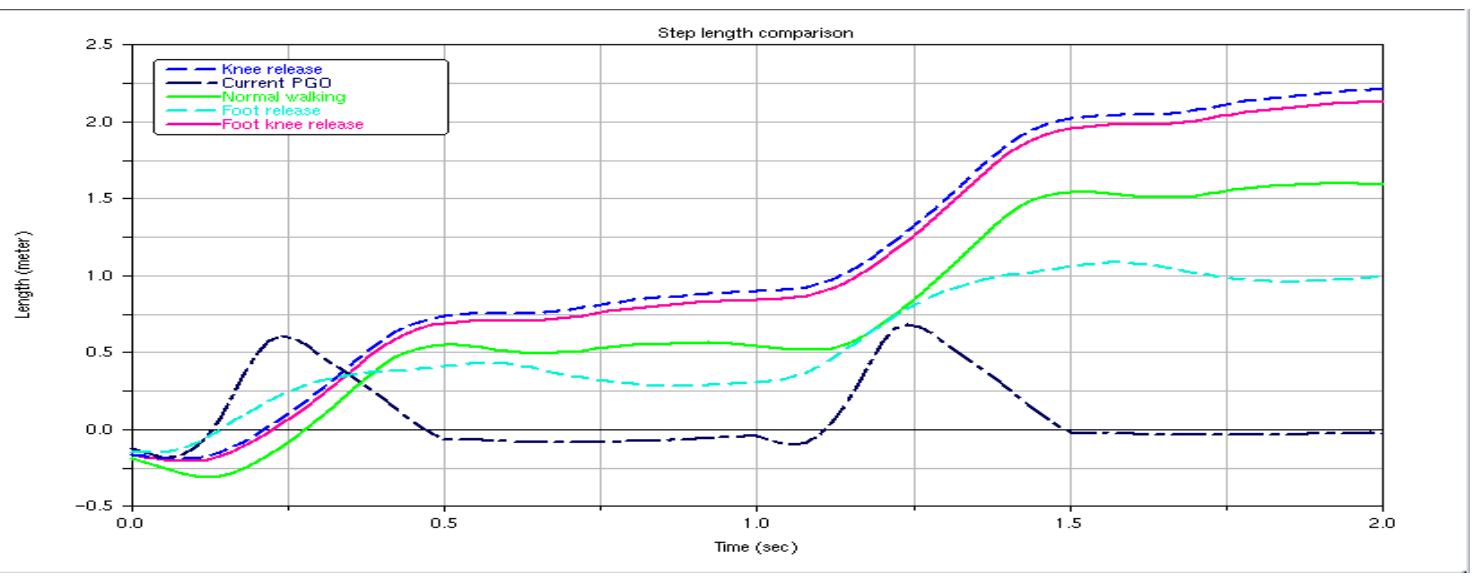

Figure (5 - 20) Comparison of stride lengths or distances

2. Clearance. The clearance is shown in Figure $(5-10)$. Before the middle swing there is bump in foot release walking. This is the result from the penetration of the other leg. 
In the simulation of normal walking the same problem happened there. In real walking, there is no penetration, therefore the clearance for swing leg is enough. In current PGO walking even though there is no bump in the swing phase, it still had a clearance problem. Because it walked in step not in stride, and only one leg moved, the trailing leg was pulled forward by the stance foot. There was no swing and thus no clearance during this period.

3. Standing position. Any walking must begin from standing. Therefore the standing position is very important for the device. As stated previously, the hip and knee are coupled. Two legs moves at the same time and in relation; thus it is coupled in some extent. With four joints coupled together, how can a standing position be achieved, with all joints angles at the same time measured as zero or close to zero? Four joint angles are shown in Figure (5 - 21). Both foot release movements are ignored because they are separated and have no effect on standing position. From Figure $(5-21)$ there is no common intersection for all four curves close to zero. That means there is no standing position for foot knee release and knee release. However, even though the two hip angle curves do not intersect at zero, they intersect at a small angle $7.8^{\circ}$. In that position the user can stand with help of hands and start his walking. This is the reason the foot release PGO is chosen to be physically realized. 


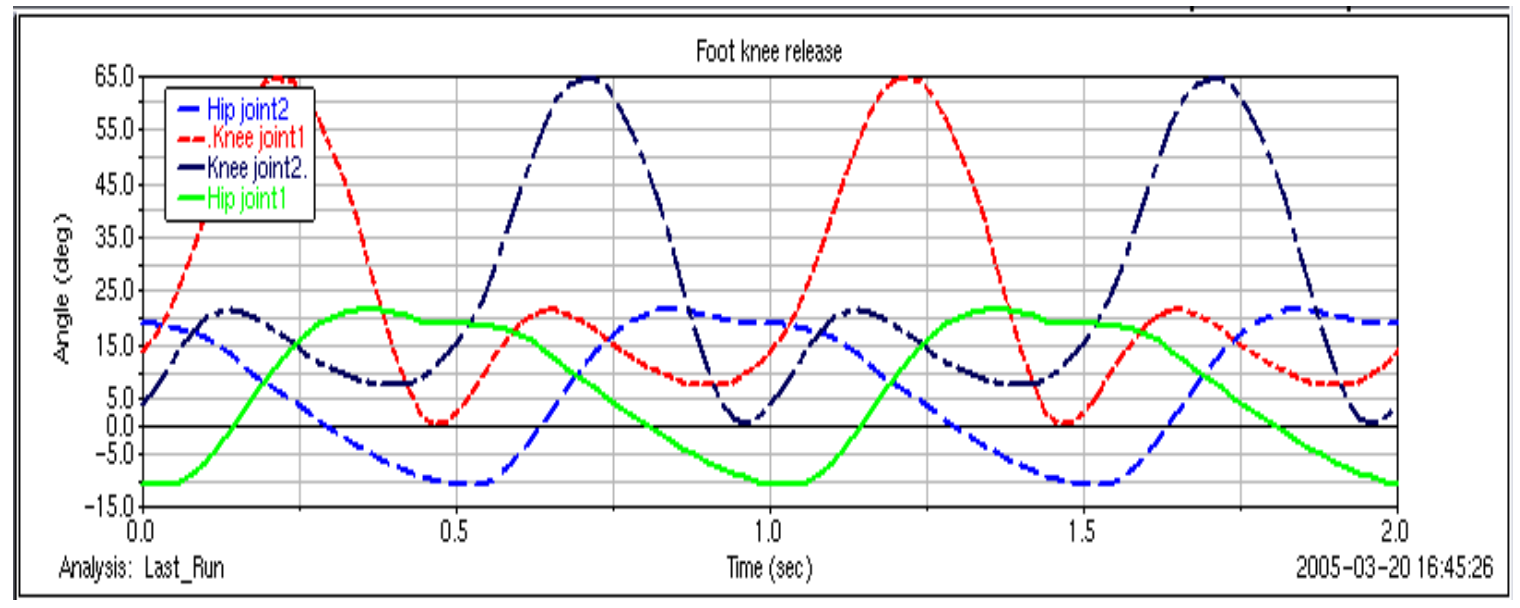

Figure (5 - 21) PGO mechanism model hip and knee angles

4. Control. From the analysis above, it is known the whole PGO mechanism has only three DOF, two foot releases (translate), and one for all hip and knee joint, regardless of foot knee release or foot release. Knee release has only one DOF. If all hip and knee are coupled, the device has no standing position. If decoupled then how are these Does controlled? From the first chapter they can be controlled by computer. That makes the device more complex. Thus only to couple two hip joints in foot release is the simplest method of control.

\subsection{Comparison of Foot Release PGO with RGO}

1. No knee movement. Both orthoses have no knee movement. Knee movement for healthy people involves making clearance and coordinates the movement of the whole body to minimize the energy consumption. However, for the handicapped, to prevent collapse of the whole body, the knee joints of the orthoses are locked during walking. 
2. Foot/ground clearance. The foot release device can retract to allow swinging leg enough foot clearance in the sagittal plane for the user walking in the PGO, while the user walking in the RGO has no clearance in the sagittal plane. The RGO user has to tilt to the stance leg to make clearance for the swinging leg.

3. Hip movement. The PGO can exactly replicate the movement of the hip joint, while in the RGO the transfer cable makes both the swinging leg and the stance leg move almost the same angle but in opposite direction mechanically. In the normal gait the positive angle (forward leg) is much greater than negative angle (trailing leg).

4. Energy consumption. The PGO user mostly consumes the external power to move forward, while the internal energy is mainly to keep the balance of the upper body. Because of the foot release device, the user does not need to tilt his body; thus the energy consumption should be low. However, the RGO user has to consume a lot of energy, because the movement of the hip joint and pelvis of RGO user is much greater than that of a healthy subject. This is of course a necessary consequence of the patient having to lift and rotate the pelvis to swing a leg through its swing phase due to the necessary locking of the knee joint. The Bowden cables on the orthosis connecting the hip joints transfer the reverse motion to the stance leg, propelling the subject forward. This action is highly energy consuming. The tilt of pelvis and the jerky gait make the center of gravity swing widely and the acceleration of the center of gravity not as smooth as those in normal walking. These motions also cause more energy consumption. 


\subsection{Investigation on the Adjustment of Step Length of Foot Release PGO}

How to easily adjust the step length to satisfy the need of the user during the PGO walking is the main goal of this part. The simple way is to change the length of one link only. However, without changing the cam profile, the hip function or other properties may be abnormal. All these need to be further investigated. First examine what happens to the hip angle when the position of jonit3 in Figure (5 - 22) move left and right just $0.2 \mathrm{~cm}$ and $0.5 \mathrm{~cm}$.

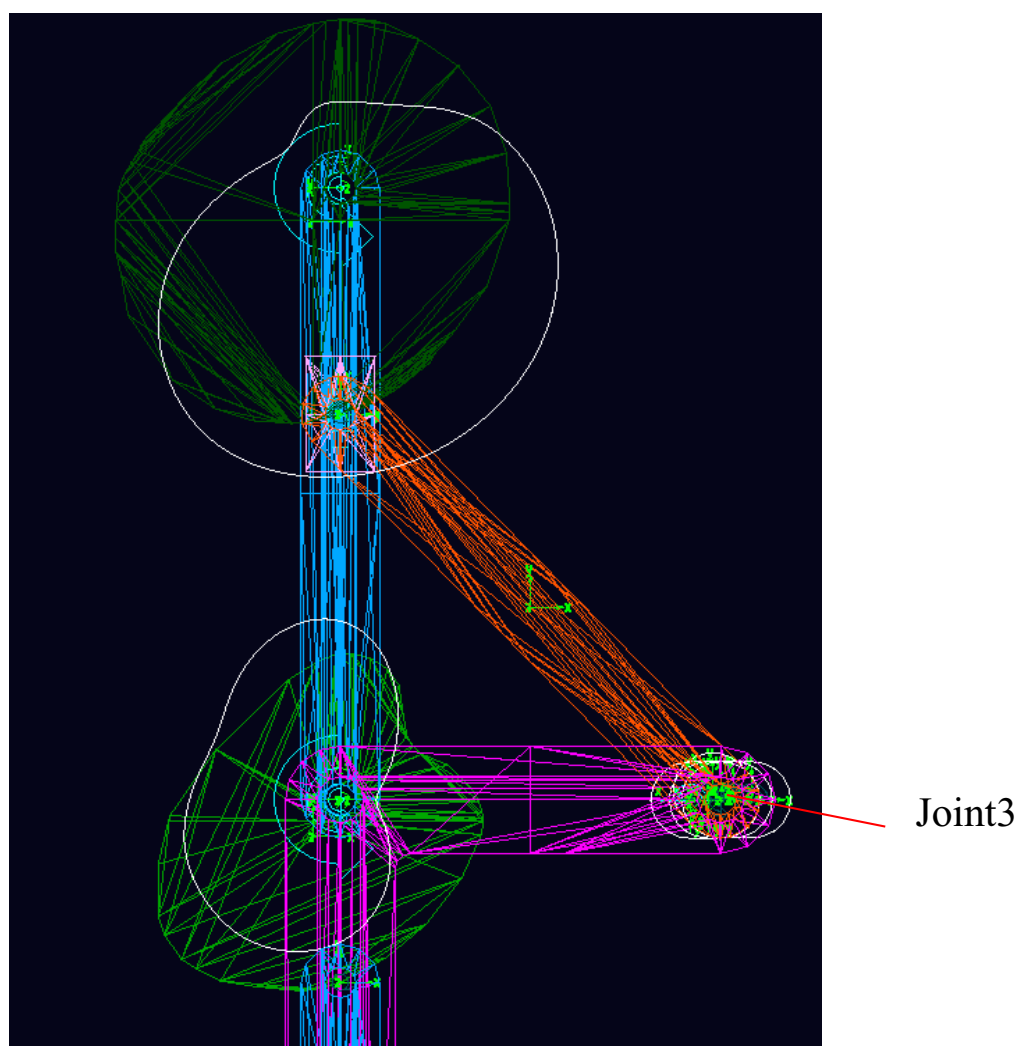

Figure (5 - 22) Hip mechanism

Corresponding hip angles are shown in Figure (5 - 23). The blue curve is the original.

When the joint moves left, i.e, the link length is shorten, the hip angle moves down. While 
the joint moves right, the hip angle moves up. When they are aligned to the start point of the original curve, the hip angle increases when the joint moves left, as does the step length. While the joint moves right, the hip angle decreases, as does the step length. The corresponding step length adjustment is shown in Figure (5 - 24). However, compared with normal walking (in page 29 of Winter's book), decreasing step length is slightly abnormal compared to slow walking (slow walking with a short stride length in page 12).

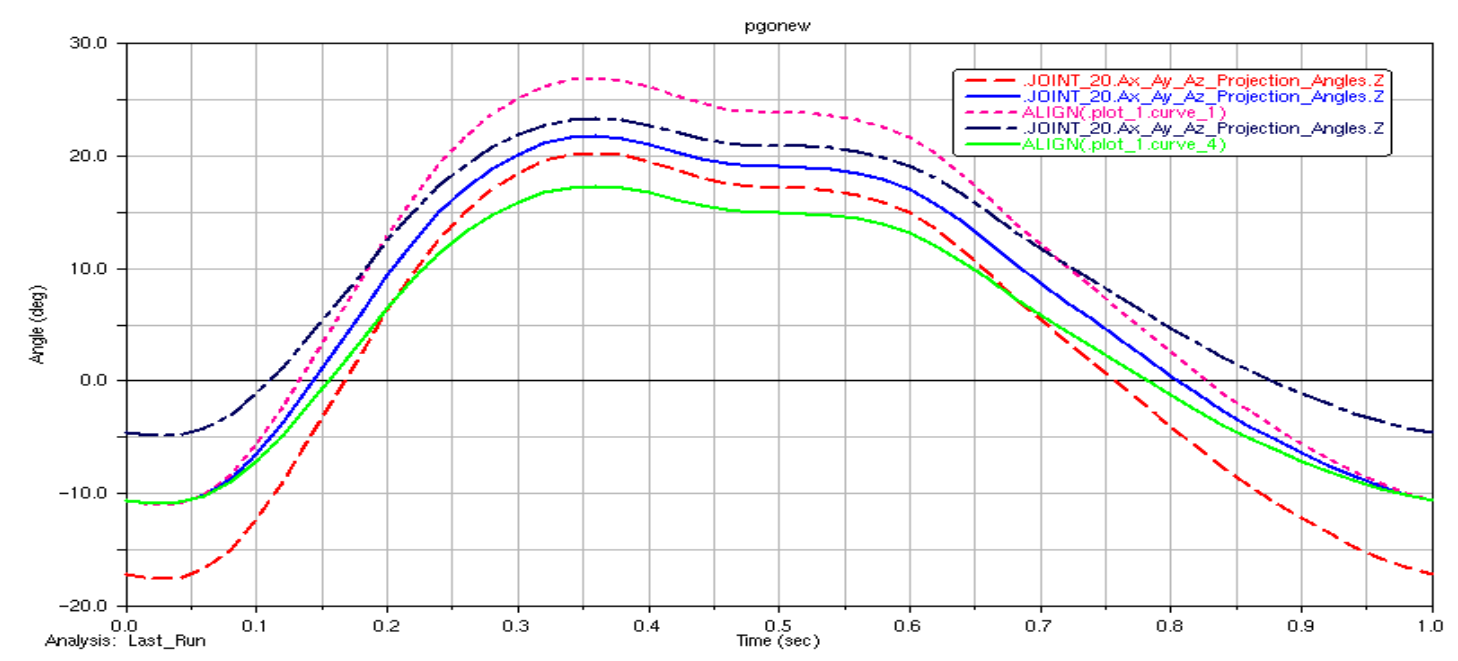

Figure (5 - 23) Adjustment of hip angles in foot release PGO

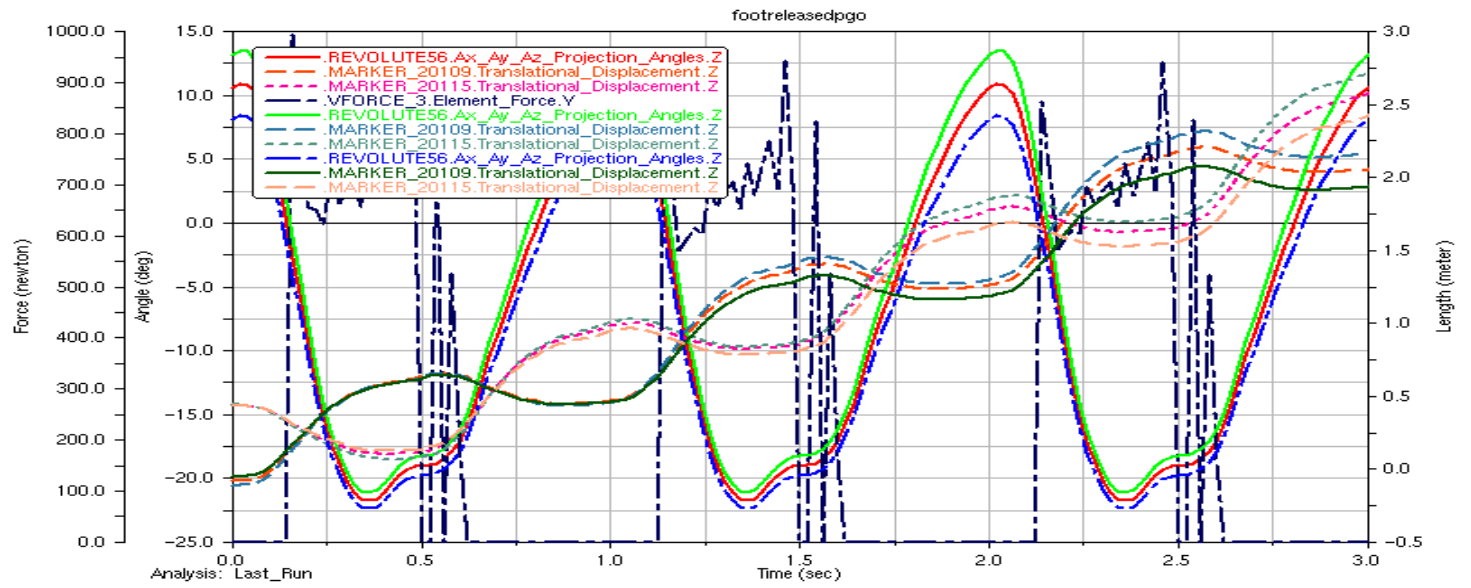

Figure (5 - 24) Adjustment of step length in foot release PGO 


\subsection{Summary and Conclusion}

\subsubsection{Summary}

1. Whatever the PGO is, it must be reciprocal.

2. Power ratio in order are: Current PGO, foot release, foot knee release, knee release and normal walking.

3. Walking in the knee release PGO looks more natural, and needs less power. However, if the knee and the hip are coupled, there is no standing position. If they are decoupled, the control system for knee is very complex.

4. The foot release PGO can only replicate hip motion. But the foot release mechanism is easier to develop than knee control system, and the user can get a standing position with the foot release PGO.

5. The foot release PGO has more advantages than RGO.

6. Step length is adjustable and with the similarity of curve shapes of hip angles through changing one link length.

\subsubsection{Conclusion}

Foot knee release and knee release are too complicated for a simple device. Even though less power ratio is needed, the hip and knee joints must be decoupled and controlled by computer. The foot release PGO is the trade-off of power ratio, standing position and control complexity. 


\section{DESIGN OF THE FOOT-RELEASE PGO}

As stated in last chapter, foot release PGO mechanism is chosen to be physically realized because of simplicity of control, standing position, even though energy cost is slightly high. In mechanism simulation, all motions just exist in mathematical equations. In this chapter the results from mechanism simulation will be used to design a virtual model and then physically realize it. The forces, motions, and power consumption from the simulation are the basis for the design.

\subsection{Design of Hip joint}

\subsubsection{Cam Follower}

From last chapter we know the hip joint is approximated by a cam-modulated slider crank mechanism. The weakest part in this mechanism is the cam follower. To ensure the follower can work properly, it must have enough strength. The cam follower's contact force shown in Figure $(6-1)$ is the basis for the design or selection of the cam follower. The highest force is $7245 \mathrm{~N}$. Then the diameter of cam follower is $0.6 \mathrm{in}$. This force is also used to determine the diameter of the cam shaft. These two dimensions are used to determine the least distance between cam follower and the rotation center of the cam. In fact, the dimension of cam follower's installation seat is also taken into account. Next the cam profile must be determined. 


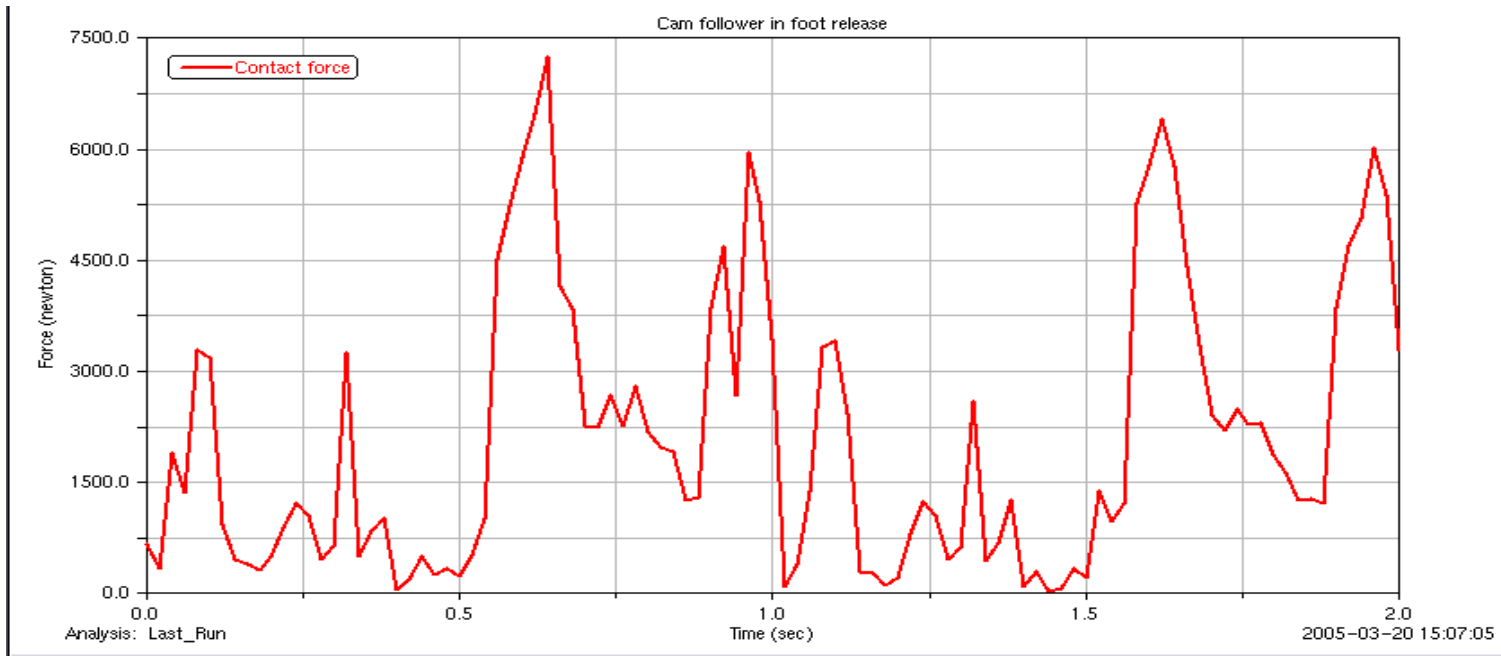

Figure (6 - 1) Contact force of cam and follower

\subsubsection{Cam Profile}

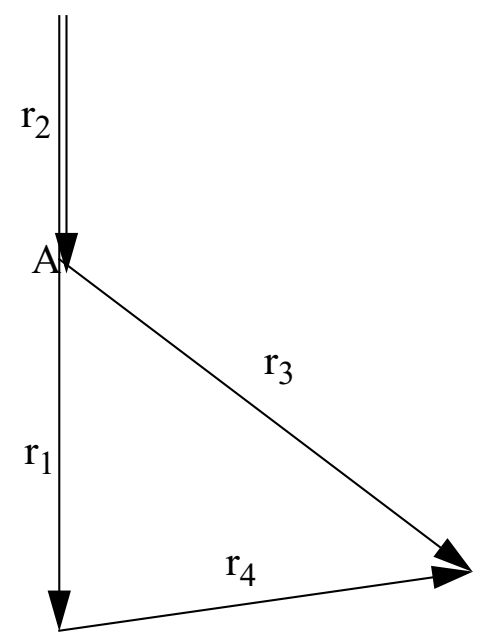

Figure (6 - 2) Vector loop

Cam profile can be calculated in Matlab by using the vector loop shown in Figure (6 2). It can also be obtained from ADAMS simulation, but link length must be determined first. The working principle of the cam slider crank mechanism is simply put as: 
Point $\mathrm{A}$ is the cam follower. It can translate along the seat's guidance $\mathbf{r}_{\mathbf{1}}$, and also have to be on the cam profile. $\mathbf{r}_{2}$ is the distance from cam center to follower. When cam is rotating, $\mathbf{r}_{2}$ is changing, then changing the angle $\theta_{4}, \theta_{4}$ is the hip angle. The loop equation is

$$
\text { Loop: } \overrightarrow{r_{2}}+\overrightarrow{r_{3}}-\overrightarrow{r_{4}}-\overrightarrow{r_{1}}=0
$$

Where $\mathbf{r}_{\mathbf{1}}$ represent seat, $\mathbf{r}_{\mathbf{2}}$ is the distance from cam center to follower, $\mathbf{r}_{\mathbf{3}}$ is just a link, $\mathbf{r}_{4}$ is for leg. $\theta_{4}$ is the hip angle. In this equation $r_{1}, r_{3}, r_{4}, \theta_{1}$ and $\theta_{2}$ are knowns, and $\theta_{4}$ is the hip angle which is the same as that used in the simulation. Now the unknowns are $r_{2}$ and $\theta_{3}$.

After many tries, $r_{1}, r_{3}$ and $r_{4}$ are determined. $r_{1}=5$ in, $r_{3}=3.7$ in, $r_{4}=2.6$ in. $X$ and $Y$ coordination of $r_{2}$ are shown in Figure $(6-3)$. This is the cam profile. The minimum $r_{2}=$ 1.2325 in, maximum $r_{2}=2.8145$ in. Maximum $r_{2}$ is used to determine the diameter of the cam.

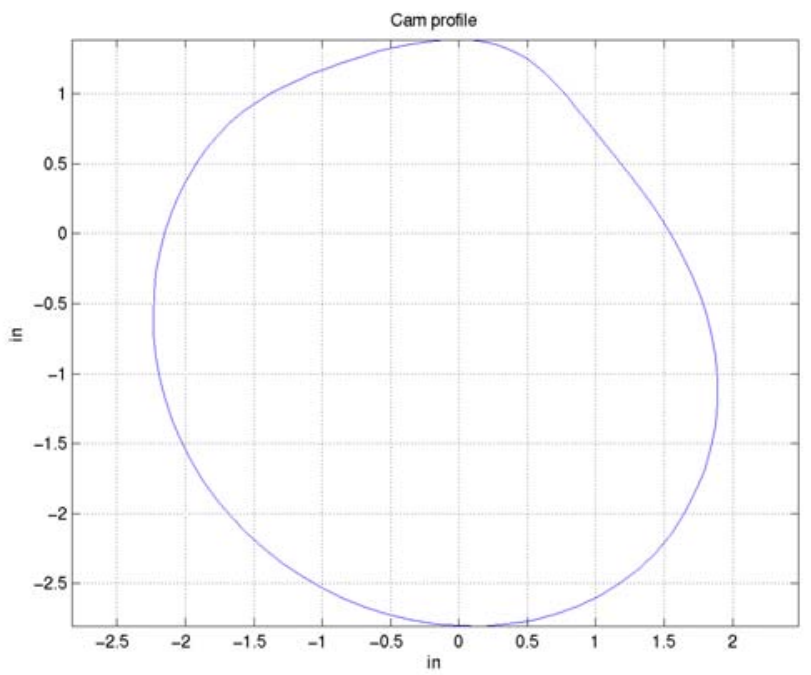

Figure (6 - 3) Cam profile 


\subsubsection{Driving Power for Cam}

From last chapter the maximum power for hip joint is $485.4 \mathrm{~W}$, average power is $81 \mathrm{~W}$, the maximum moment $77 \mathrm{Nm}$, the average moment is $12.9 \mathrm{Nm}$. These data can be used to select motor and decide the ratio of the worm gear reducer. But here the motors was the same as that used in current PGO. The hip virtual model is shown in Figure (6 - 4). The maximum contact force between the cam and the follower will be checked in the later simulation.

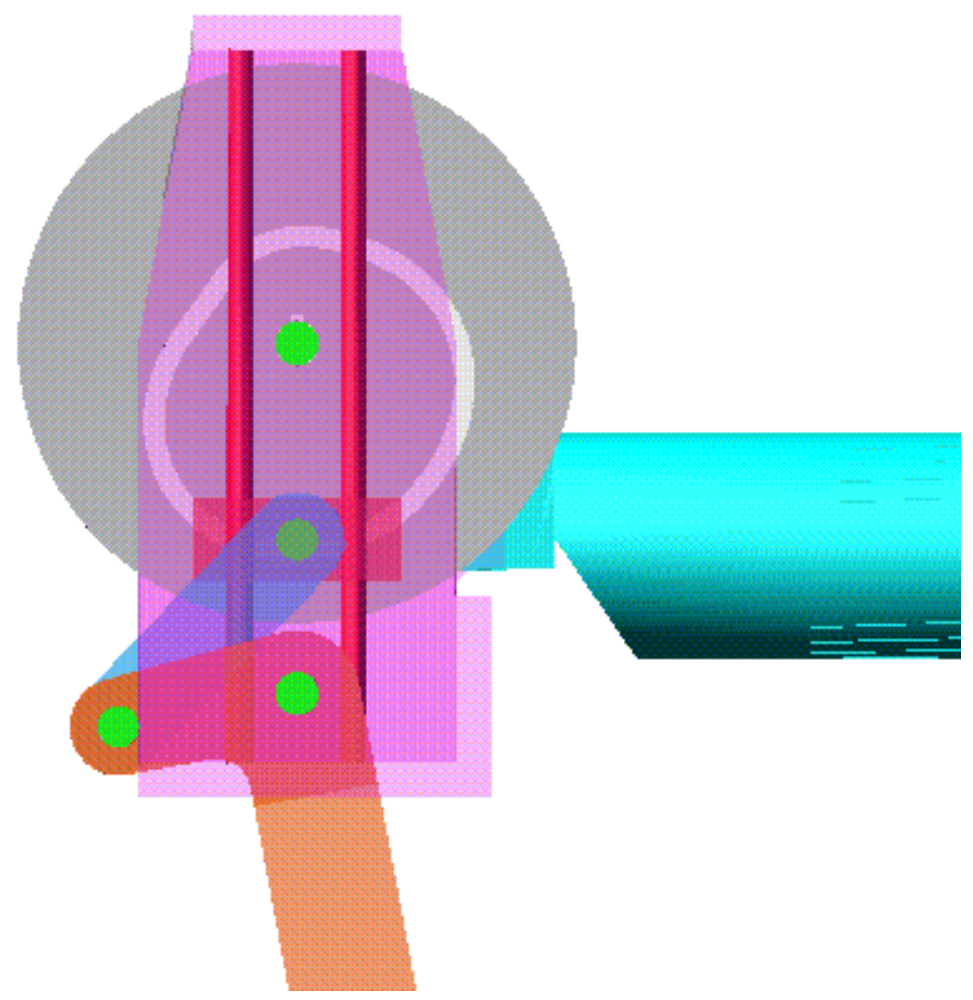

Figure (6 - 4) Hip virtual model 


\subsection{Design of Foot Release}

\subsubsection{Release Mechanism}

Foot release mechanism only works at the swing phase of the leg. When the user needs to swing his either leg to move forward, the length of the swing leg must be shortened first to make clearance for the swing leg. Right before the swing leg contacts the floor the foot release is extended to its original length to prepare for stance phase. Therefore, there is no force acting on the mechanism except for the weight of itself. The power to drive the mechanism is very small. However, the mechanism must have enough strength to bear the weight of the user and the impact force between the foot and floor occurred in walking. In addition, the mechanism must be self locked at any position to keep the stability of the user in any situation. The requirements for the foot mechanism can be simply put as:

- The thickness is as small as possible

- The ratio of contraction to extension the higher the better

- Self lock is needed at any position

- The weight must be small

- The power source can be easily carried and controlled by the user

The mechanism used in the previous simulation is shown in Figure (6 - 5). The big advantage of this mechanism is that it reacts very quickly. However, it is not self locked, and the power source is fluid and thus not easy to carry. If an electrical cylinder is used then its quickness is gone. Therefore a new mechanism is developed. A self lock worm gear is used to drive a crank slider to change thickness of the foot plates. The model is shown in Figure (6 - 6). The main features of this mechanism are as: 
- The thickness is $100 \mathrm{~mm}$

- The contraction ratio is $30 / 100$

- Worm gear self lock

- Power source is battery

- Step motor to control the position

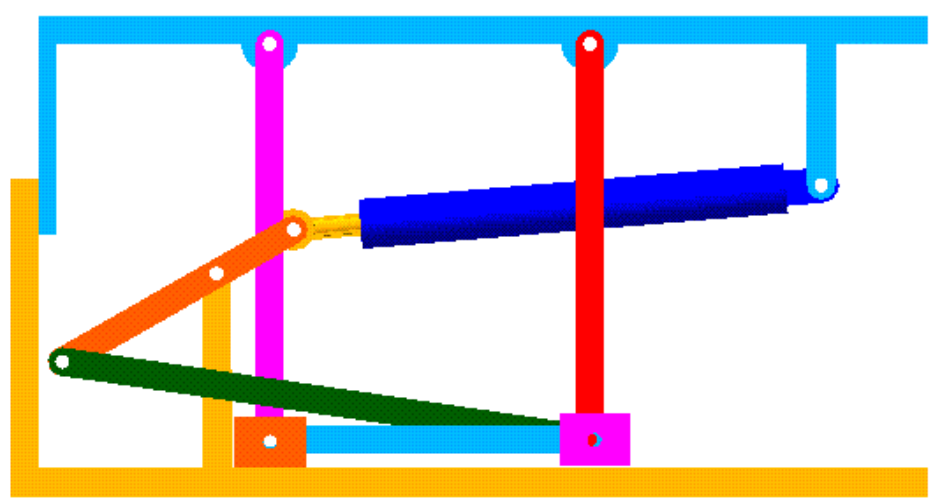

Figure (6 - 5) Cylinder release mechanism

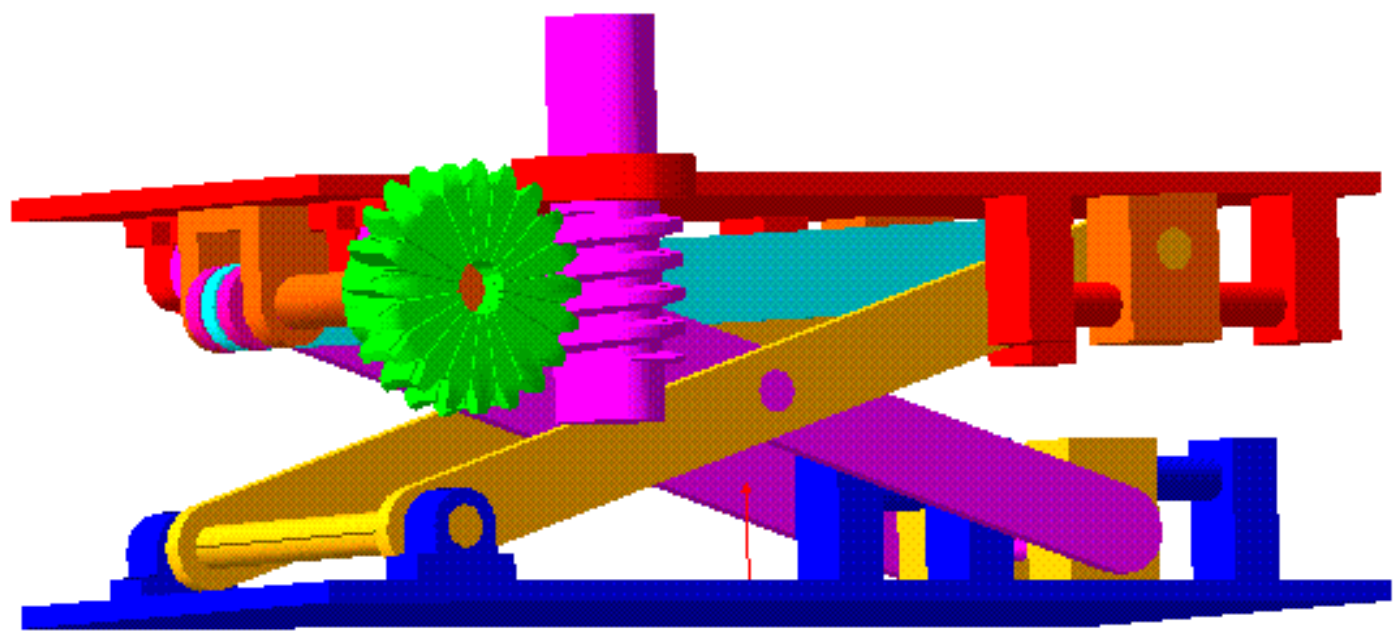

Figure (6 - 6) Worm gear release mechanism 


\subsubsection{Worm Gear}

Because there is no force acting on it when the worm gear is working, the power to drive can be very small. However, during the stance phase when the user is standing on it, the gear must have enough strength to overcome the locking force. The worm gear can not be selected just according to the transfer power or torque when it is running. They must be selected by checking the strength on self locking. The gear strength at this moment was checked by the equation for the momentary overload capacity of worm gear (Stokes, A., 1992). Now the model with new foot release is simulated and the running torque on the gear are shown in Figure (6 - 7). The maximum torque is $9.68 \mathrm{Nm}$. As said above this torque can not be used to choose worm gear. A self locking torque must be found on the gear. To obtain maximum self locking, the foot release was simulated with maximum ground reaction force acting on it in case it will stop at any position and still have enough strength. The maximum ground reaction force is twice what obtained from the simulation of worm gear release in case where the weight is above normal. It is $2200 \mathrm{~N}$. Torque curve from single foot release simulation is shown in Figure (6 - 8).

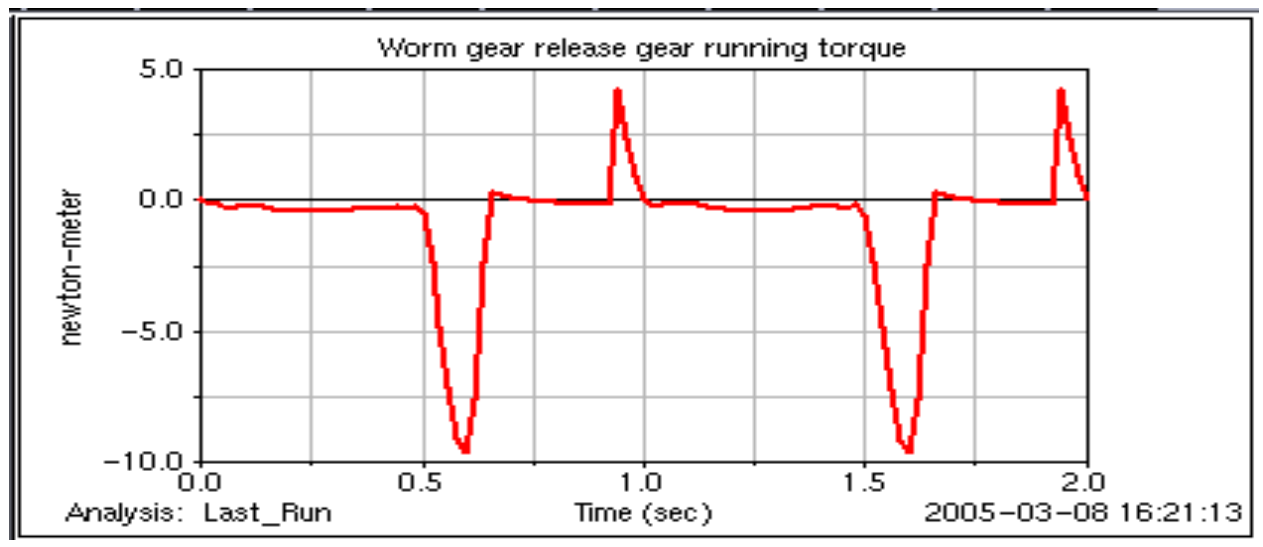

Figure (6 - 7) Torque on gear in worm gear release model 


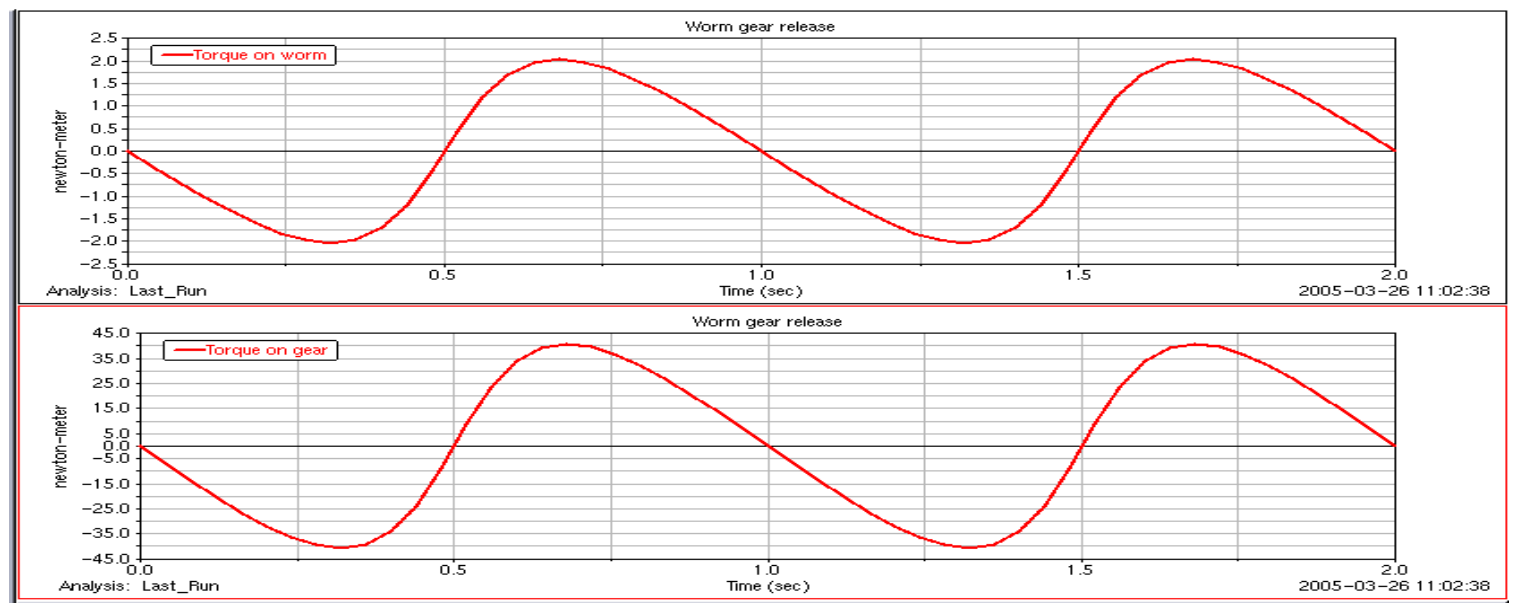

Figure (6 - 8) Torque from single worm gear release simulation

The maximum torque on gear is $40.8 \mathrm{Nm}$ or $361 \mathrm{lb}$.in. From Martin worm gear chart we select a pair with self locking (helix angle less than $5^{\circ}$ ), ratio 20 , center distance 1.625 in and output torque $470 \mathrm{lb}$. in. at $100 \mathrm{rpm}$. The parameters for worm and gear and gear strength check are as below:

$\mathrm{N}_{\mathrm{g}}: 20$, pressure angle: $14.5^{\circ}$, pitch diameter: 2 in,

$\mathrm{N}_{\mathrm{w}}: 1$, helix angle: $4^{\mathrm{O}} 34^{\prime}$, pitch diameter: 1.25 in.

Equation of momentary overload capacity is:

for strength: $4 \mathrm{~S}_{\mathrm{bw}} \mathrm{l}_{\mathrm{r}} \mathrm{D}_{\mathrm{f}} \mathrm{m} \cos (\lambda)(\mathrm{lbf} . \mathrm{in})$

$\mathrm{S}_{\mathrm{bw}}--$ bending stress factor (for bronze wheel, $\mathrm{S}_{\mathrm{bw}}=10000$ )

$1_{\mathrm{r}}$-- length of root wormwheel tooth (linear picth*0.3686)

$\mathrm{D}_{\mathrm{f}}$-- wormwheel reference circle diameter

m -- axial module

$\lambda$-- lead angle of worm thread

$\mathrm{p}=\mathrm{Dg} * \pi / \mathrm{Ng}=2 \pi / 20=0.3142 \%$ 


$$
\begin{aligned}
& \mathrm{m}=\mathrm{p} / \pi=0.1 ”=2.54 \mathrm{~mm} \\
& \mathrm{l}_{\mathrm{r}}=0.3142 * 0.3683=0.1157^{\prime \prime} \\
& \mathrm{T}=4 * 10000 * 0.11570 * 2 * 0.1^{*} \cos 4^{\circ} 34^{\prime}=923 \mathrm{lbf} . \mathrm{in}=104 \mathrm{Nm}
\end{aligned}
$$

Then the Safety Factor is $104 / 40.8=2.55$. It seams a smaller one with the center distance 1.333 can be used.

\subsubsection{Driving Power for Foot Release}

The simulated torque and power on worm is shown in Figure (6 - 9). These are the basis for selecting driving power of foot release. The maximum power is $51.7 \mathrm{~W}$, the maximum torque is $0.48 \mathrm{Nm}, 68.56$ ounce.in.

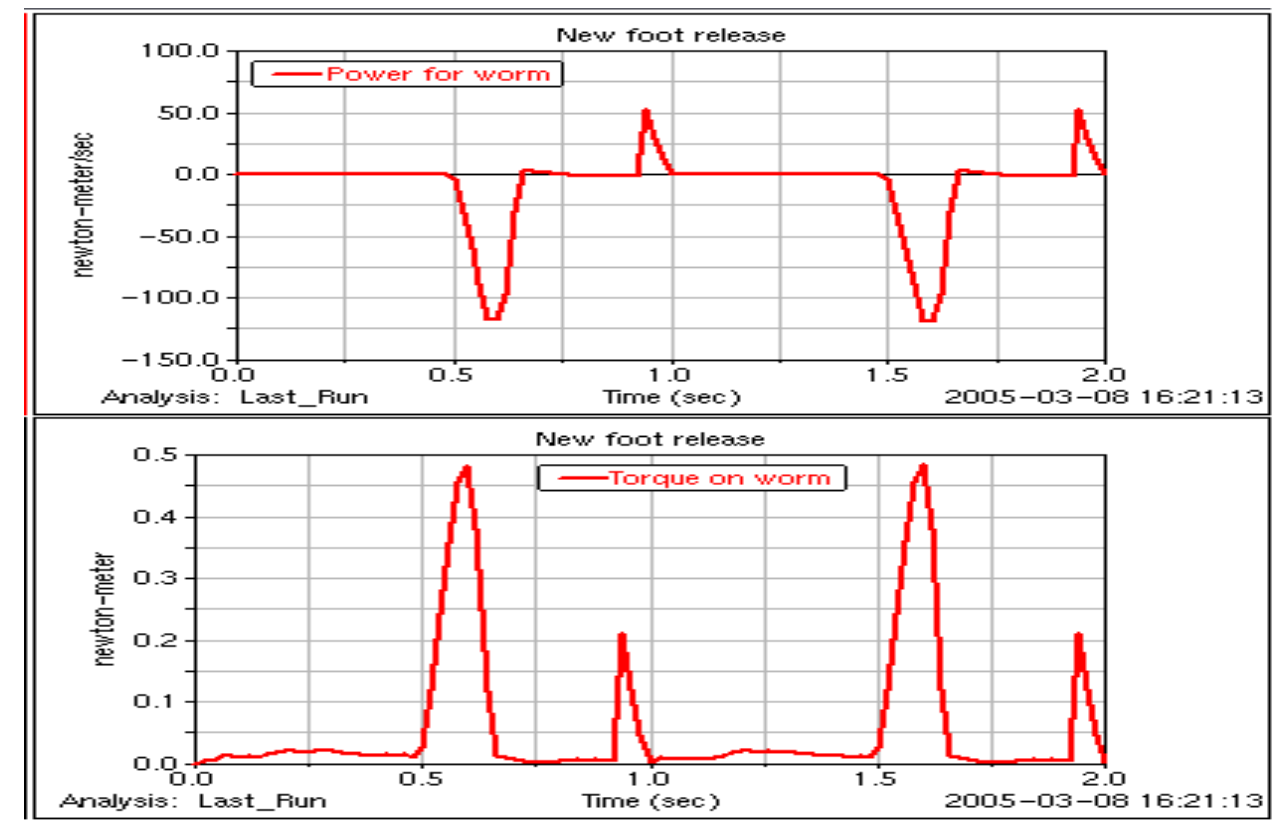

Figure (6 - 9) Driving torque and power on worm

Because the position of the low footplate worked always in two extreme positions, a step motor was needed. AM23-150-2 step motor and DAX controller are selected from 
Advanced Micro Systems Inc. The maximum output torque is over $80 \mathrm{oz}$.in. The foot release virtual model is shown in Figure (6 - 10).

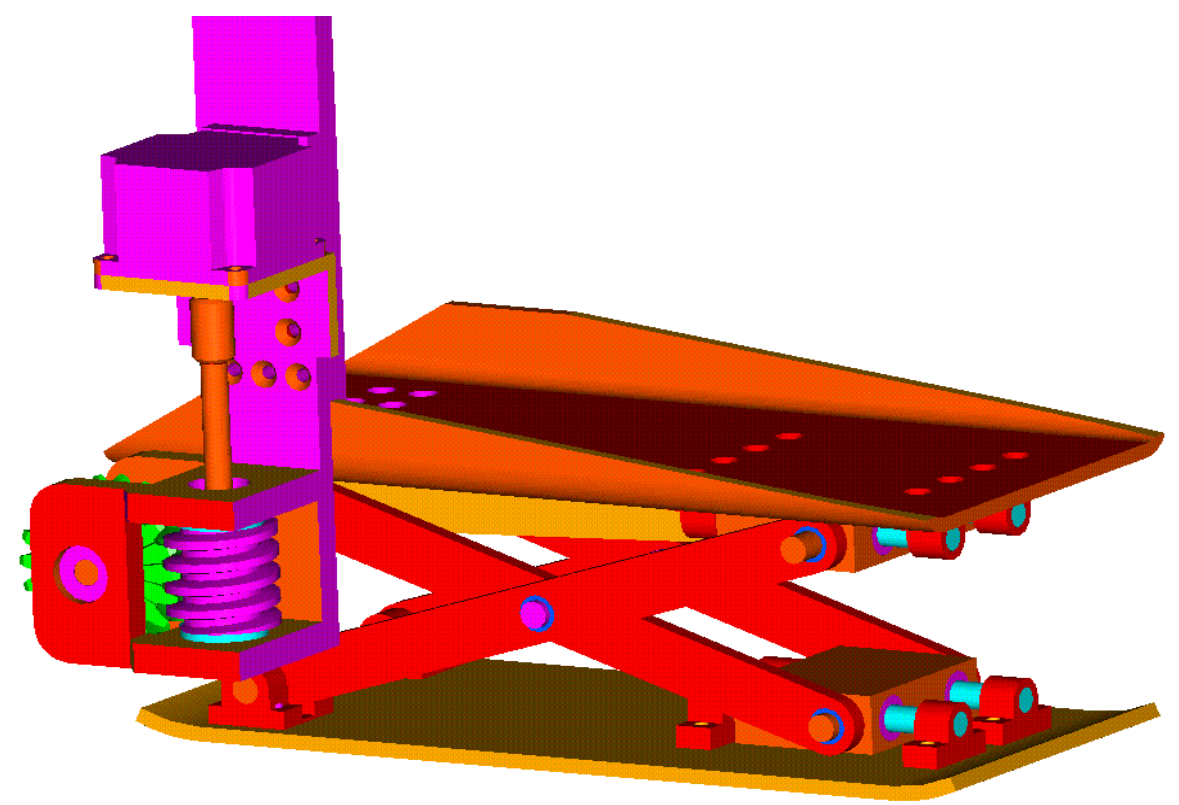

Figure (6 - 10) Foot release virtual model

\subsection{Foot Release PGO Virtual Model}

Put the hip joint and foot release together, it is one leg of the PGO virtual model. Then make a copy, rename it and mirror it in sagittal plane, to obtain the other leg. The final PGO virtual model is shown in Figure (6 - 11). This model did not include power source and controller. They can be added on the frame used to connect two legs. The knee joint is locked during walking, and can be unlocked when the patient needs to sit down. 


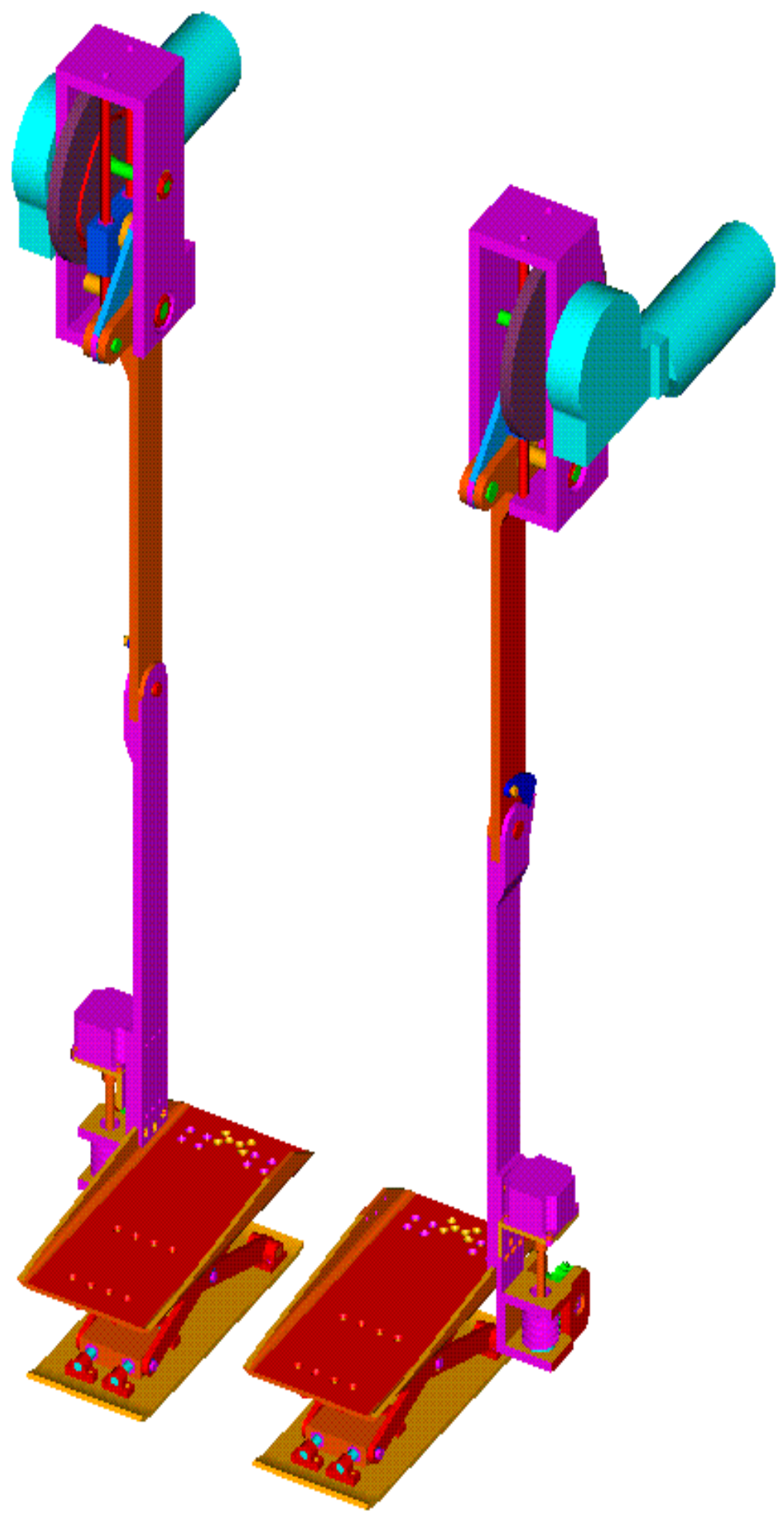

Figure (6 - 11) Foot release PGO virtual model






\subsection{Simulation of Foot-release PGO Virtual Model}

Before it is made into a prototype, the foot release PGO virtual model is simulated. The main reasons are:

- To ensure the key parts do not fail

- To ensure the power and torque required are under the power supply

- To check the function of the final PGO virtual model after modification in the design.

- To prepare calculated results for experimental validation

The simulation is shown in Figure (6 - 12).

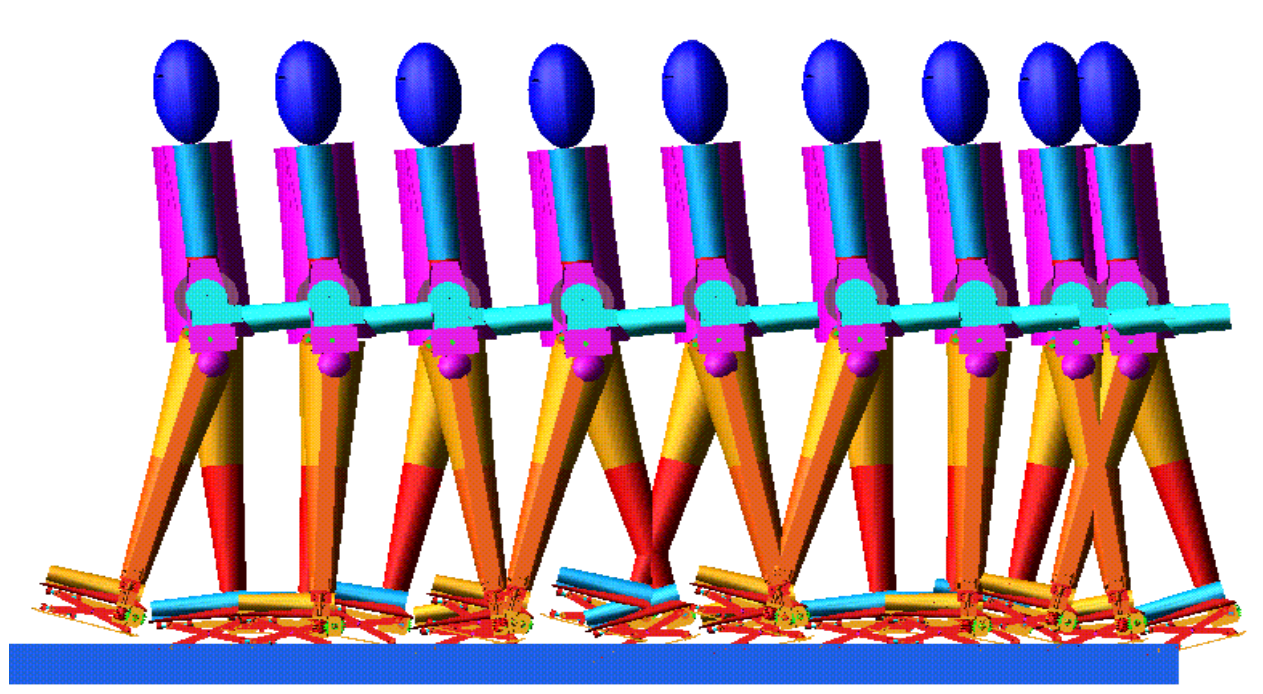

Figure (6 - 12) Simulation of Foot release PGO virtual model 


\subsection{Analysis of Simulation Results}

\subsubsection{Joint Motions}

Joint motions are shown in Figure (6 - 13). A constant velocity of hip motor gear can produce exact hip motion through the cam-modulated slider crank mechanism. The velocity for release motor is not continuous, but easily controlled by hand. It is approximated by Fourier series in simulation. Compared with cylinder release shown in Figure (6 - 14), the velocity of virtual model has the similar curve form with the displacement of cylinder release. That make the responding velocity of the virtual model smaller than that of the cylinder release. The maximum responding velocity is shown in Table (6 - 1) on page 115 . If the displacement of virtual model like that of cylinder release is wanted, then the motor speed can be increased and stopped at the maximum release displacement. However, the step motor can not respond so quickly.

Table (6 - 1): Comparison of Cylinder and Worm Gear Release

\begin{tabular}{c|c|c|c}
\hline & $\begin{array}{c}\text { Responding } \\
\text { velocity Max. (m/s) }\end{array}$ & $\begin{array}{c}\text { Release } \\
\text { displacement }(\mathrm{m})\end{array}$ & $\begin{array}{c}\text { Width of curve } \\
\text { form }(\%)\end{array}$ \\
\hline Virtual model & 0.24 & 0.031 & 36 \\
\hline Cylinder release & 0.82 & 0.0385 & 44 \\
\hline
\end{tabular}




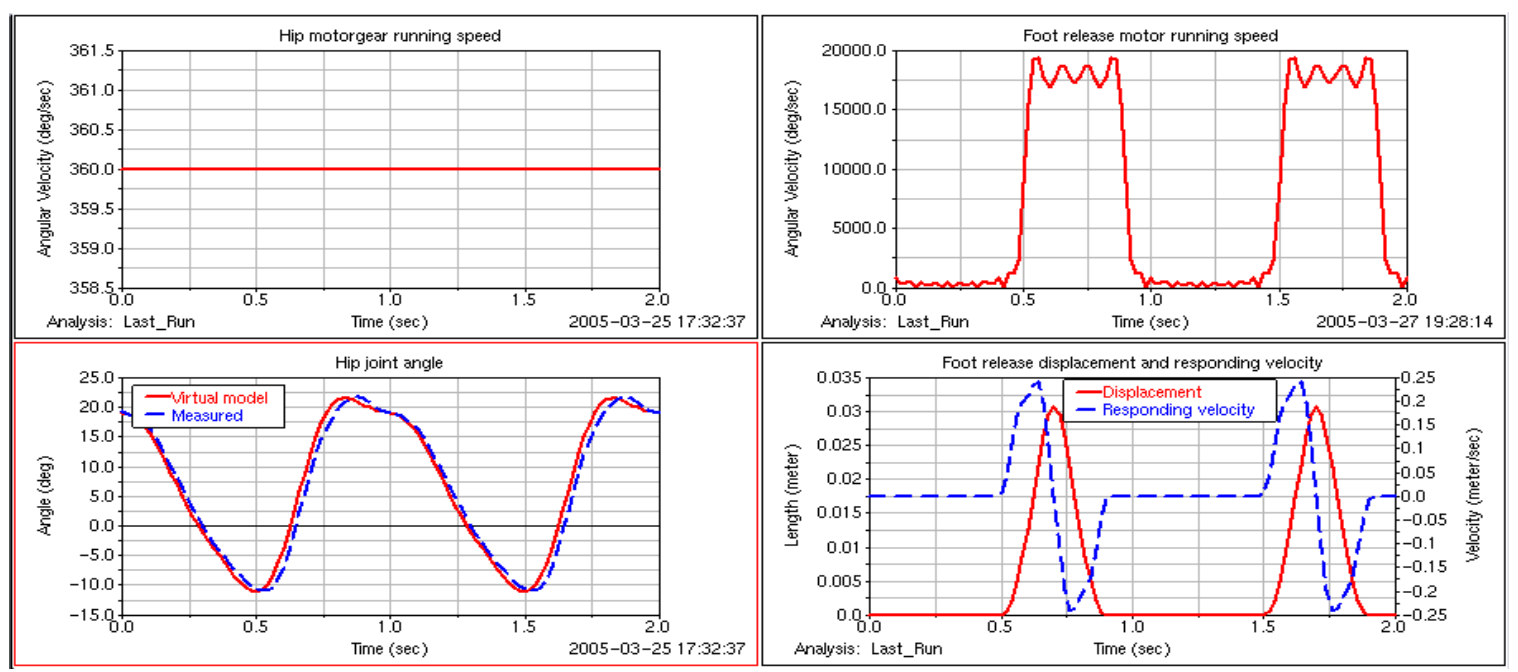

Figure (6 - 13) Joint motions in virtual model

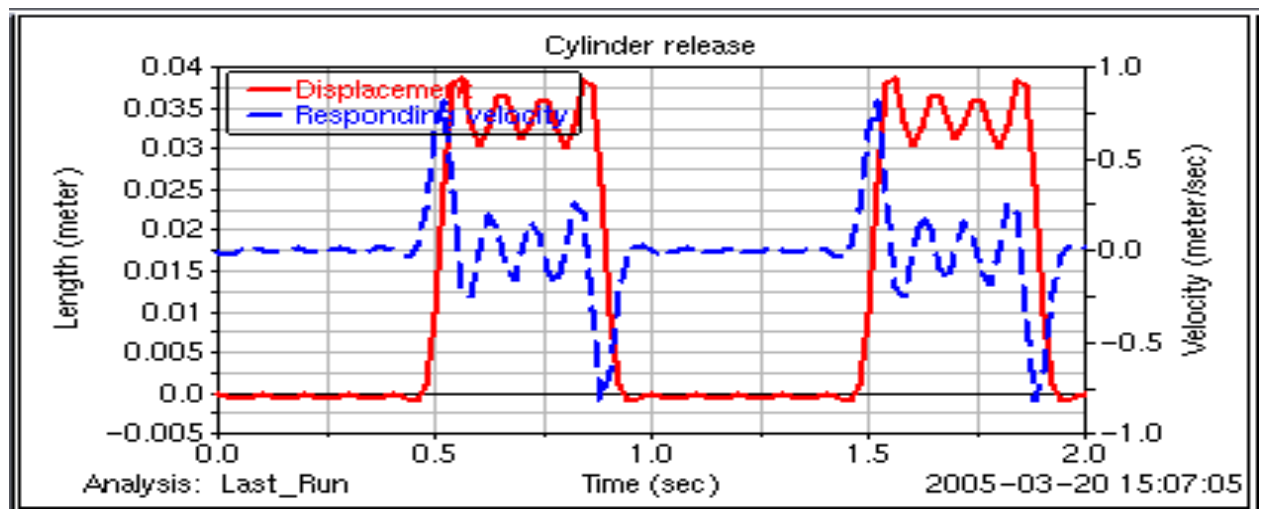

Figure (6 - 14) Cylinder release displacement

\subsubsection{Ground Reaction Force}

Ground reaction force is shown in Figure (6 - 15). Compared with that of the cylinder release model, stance phase of the virtual model is longer. The detailed data is put in Table (6 - 2) on page 117 . The average force increase shows that the virtual model has more weight than the mechanism model. The distinct increase in stance phase may result from the difference of release mechanism. As it can be seen from joint motions that both 
the responding velocity and the width of curve form have an effect on the length of stance phase.

Table(6 - 2): Maximum force and Stance phase of Virtual Model

\begin{tabular}{c|c|c|c}
\hline & $\begin{array}{c}\text { Maximum } \\
\text { Force }(\mathrm{N})\end{array}$ & $\begin{array}{c}\text { Average } \\
(\mathrm{N})\end{array}$ & $\begin{array}{c}\text { Stance Phase } \\
(\% \text { of cycle })\end{array}$ \\
\hline Virtual model & 1151.9 & 463.9 & 74 \\
\hline Cylinder release & 988.9 & 390.2 & 60 \\
\hline
\end{tabular}

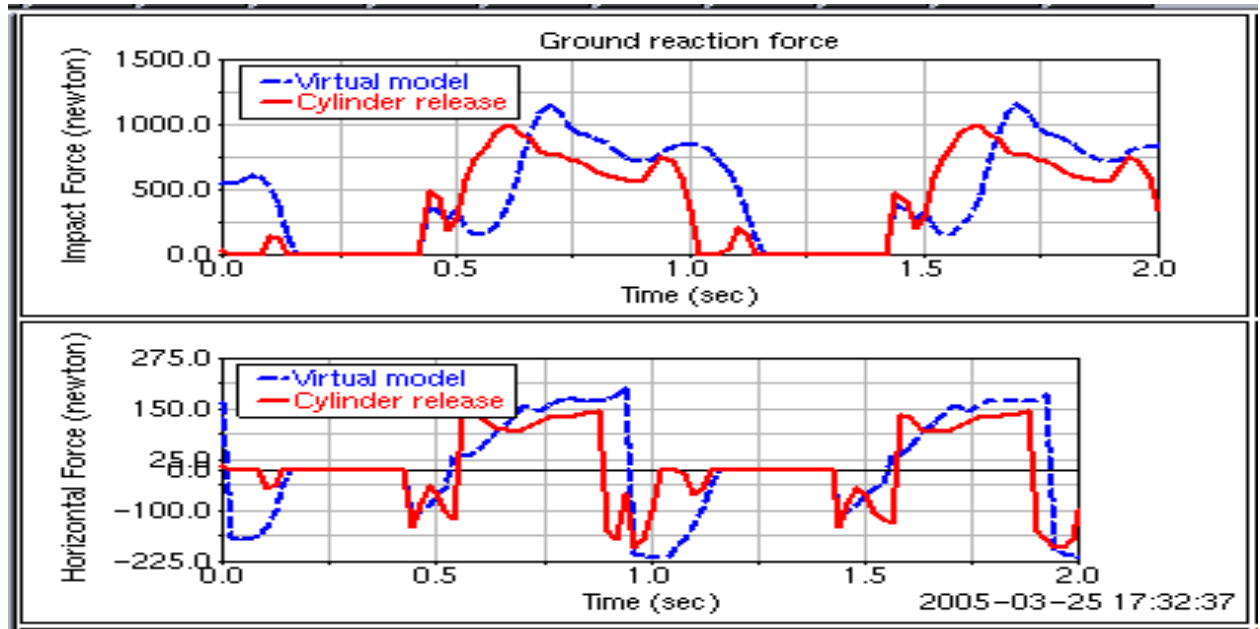

Figure (6 - 15) Ground reaction force in virtual model

\subsubsection{Sliding and Penetration}

Sliding and penetration are shown in Figure (6 - 16). Sliding here decreases the stride length. Penetration decreases the clearance of stance phase. Because of penetration, the slow release response will increase the length of stance phase. 


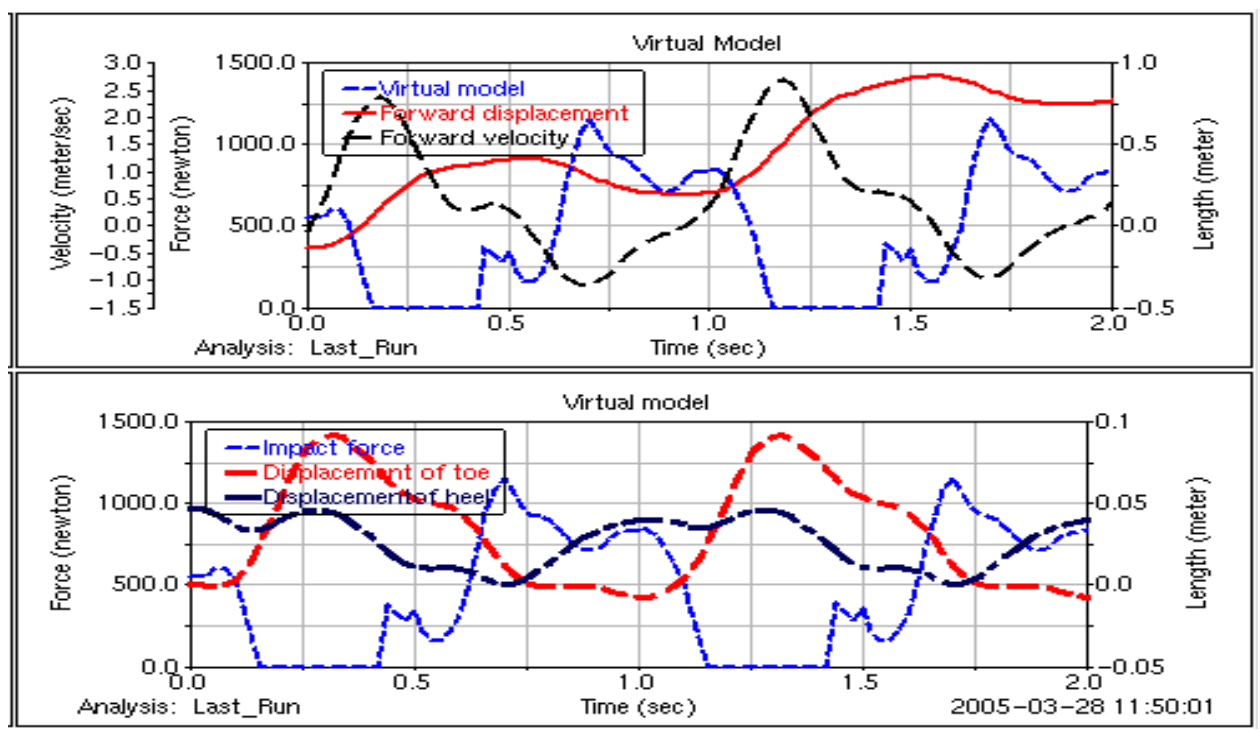

Figure (6 - 16) Sliding and penetration of virtual model

\subsubsection{The Center of Mass}

The COM is shown in Figure (6 - 17). The oscillation is smaller than that in cylinder release. The length of the leg and the stance phase may cause this difference.



Figure (6 - 17) COM of virtual model and cylinder release 


\subsubsection{Moment and Power Consumption}

The moment and power for both hip and foot release are shown in Figure (6 - 18). The data from this figure is put in Table $(6-3)$ on page 119. Compared with cylinder release, there are almost no difference in maximum moment and power for hip joint, but averages increase. That may be caused by an increase of the weight and stance phase. The maximum moment for foot release is $0.52 \mathrm{Nm}$ or $74.3 \mathrm{oz}$.in., while still under the selected step motor supply.

Table (6 - 3): Moment and Power for Virtual Model

\begin{tabular}{c|c|c|c|c}
\hline \multirow{2}{*}{} & \multicolumn{2}{|c|}{ Moment $(\mathrm{Nm})$} & \multicolumn{2}{c}{ Power } \\
\cline { 2 - 5 } & Max & Ave & Max & Ave \\
\hline Hip & 77.5 & 23.3 & 486.8 & 97.5 \\
\hline Foot release & 0.52 & 0.046 & 2.7 & -13.9 \\
\hline
\end{tabular}
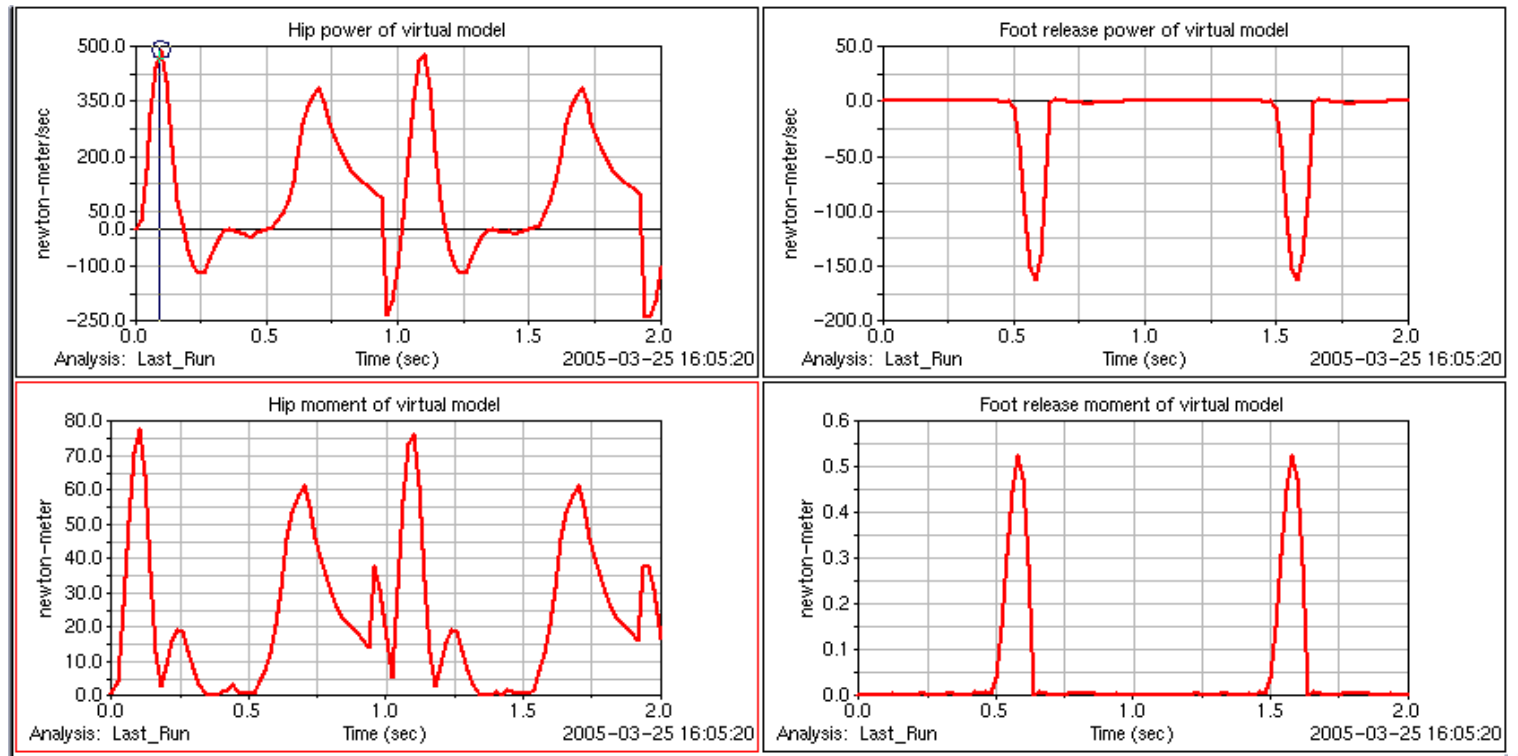

Figure (6 - 18) Moment and power in virtual model 


\subsubsection{Contact Force between Cam and Follower}

The contact force between cam and follower is shown in Figure (6 - 19). The maximum force is $7044.4 \mathrm{~N}$. This force is little bit smaller than that $(7245 \mathrm{~N})$ used to design the follower. That means follower should have the strength we desired.

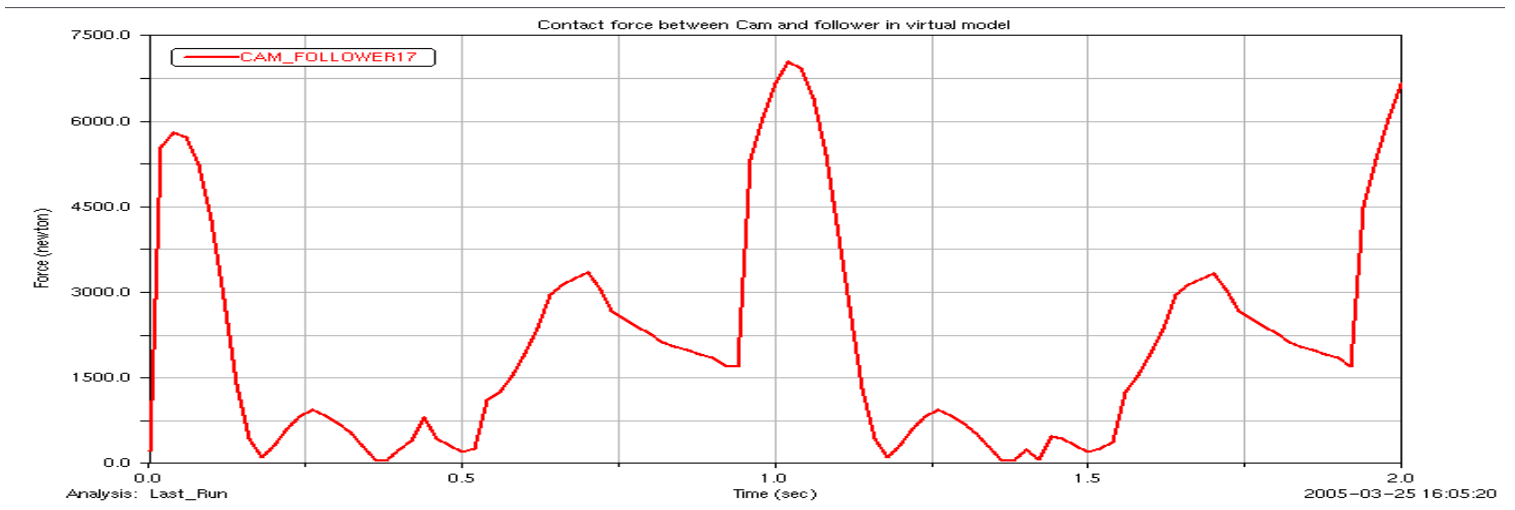

Figure (6 - 19) Contact force of cam and follower in virtual model

\subsubsection{Moment on Gear}

The moment on gear is shown in Figure (6 - 20). The maximum torque is $10.5 \mathrm{~N}$. This value is a little bit higher than $9.68 \mathrm{~N}$. This moment is running moment. It is smaller than the self locking moment. And you know the self locking torque used for design is 40.8 Nm. Thus the gear also have enough strength. 


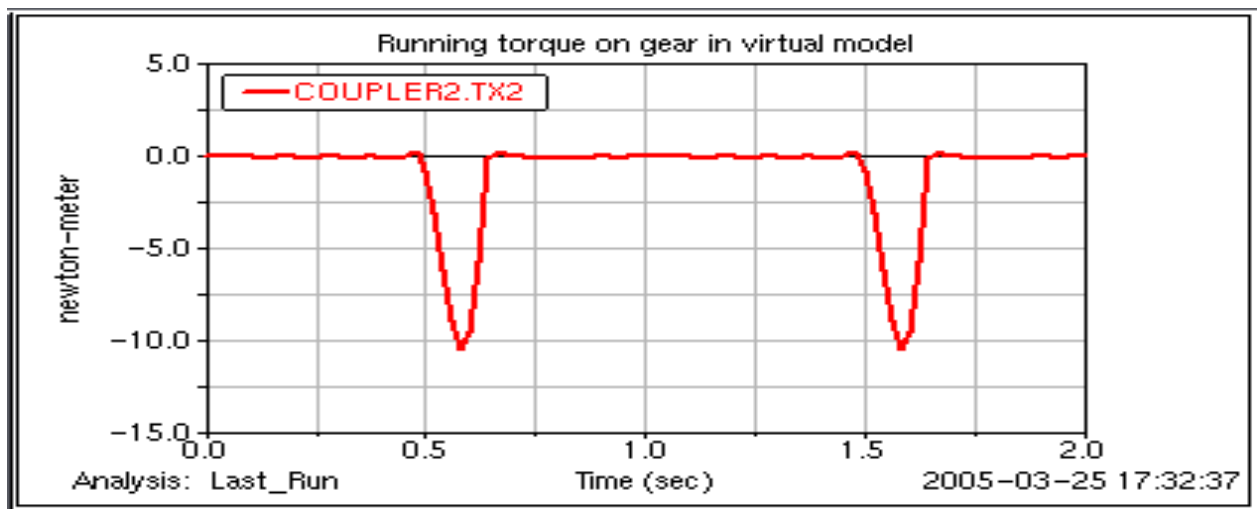

Figure (6 - 20) Running torque on gear in virtual model

\subsubsection{Power Ratio}

Power ratio is used to measure the walking efficiency. To get the power ration the walking distance is needed. The step and distance are shown in Figure (6 - 21). The data is shown in Table (6 - 4) on page 122. The stride length is almost the step length. That means the sliding has a big affect on the stride, and slow release response causes contact with the ground even when it is in initial swing. In real walking it will slow down the pace because of slow release movement.

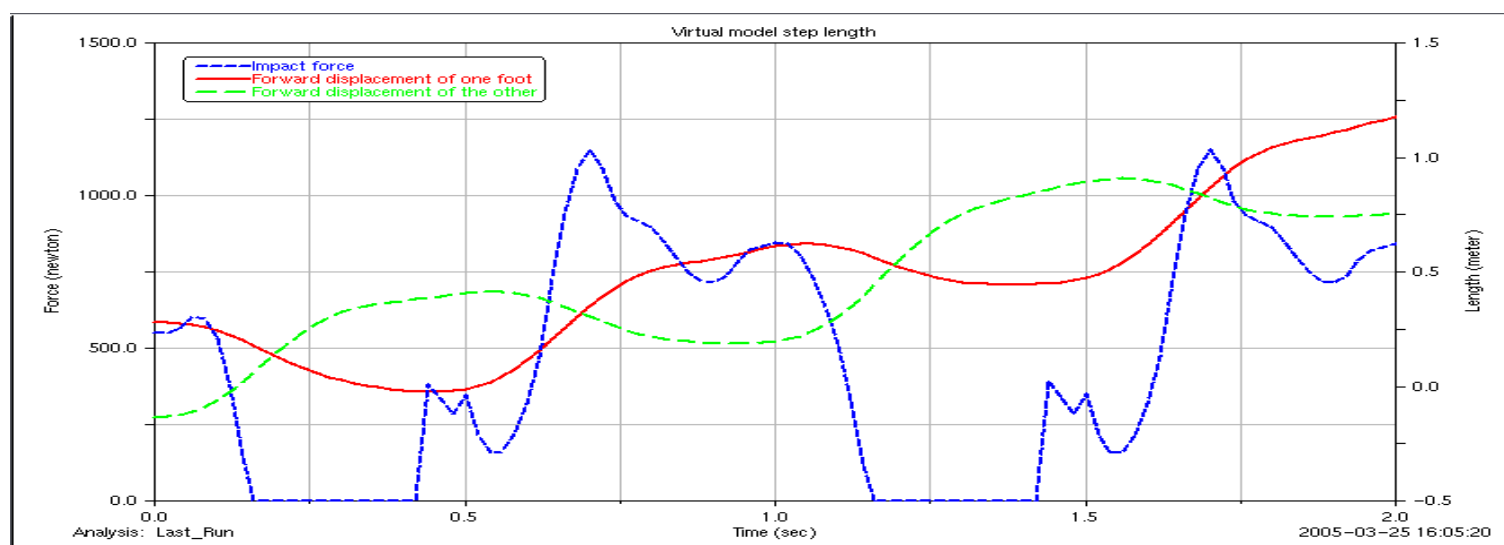

Figure (6 - 21) Step length of virtual model 
Table(6 - 4): Power Ratio of Cylinder Release and Virtual Model

\begin{tabular}{c|c|c|c|c}
\hline & Step $(\mathrm{m})$ & Stride $(\mathrm{m})$ & $\begin{array}{c}\text { Distance in 2 } \\
\text { sec. }(\mathrm{m})\end{array}$ & $\begin{array}{c}\text { Power ratio } \\
(\mathrm{W} / \mathrm{m})\end{array}$ \\
\hline Cylinder release & 0.394 & 0.703 & 1.1364 & 142.56 \\
\hline Virtual model & 0.401 & 0.403 & 0.895 & 217.88 \\
\hline
\end{tabular}

\subsubsection{Control Torque}

The control torque is shown in Figure $(6-22)$. The data is shown in Table (6 - 5) on page 122 .

Table(6 - 5): Control Torque of Virtual Model

\begin{tabular}{c|c|c|c}
\hline & Maximum $(\mathrm{Nm})$ & Minimum $(\mathrm{Nm})$ & Average $(\mathrm{Nm})$ \\
\hline Sagittal & 311 & -466 & 12.7 \\
\hline Coronal & 226.7 & -213.3 & -5.37 \\
\hline Transverse & 83.4 & -77 & -0.3 \\
\hline
\end{tabular}

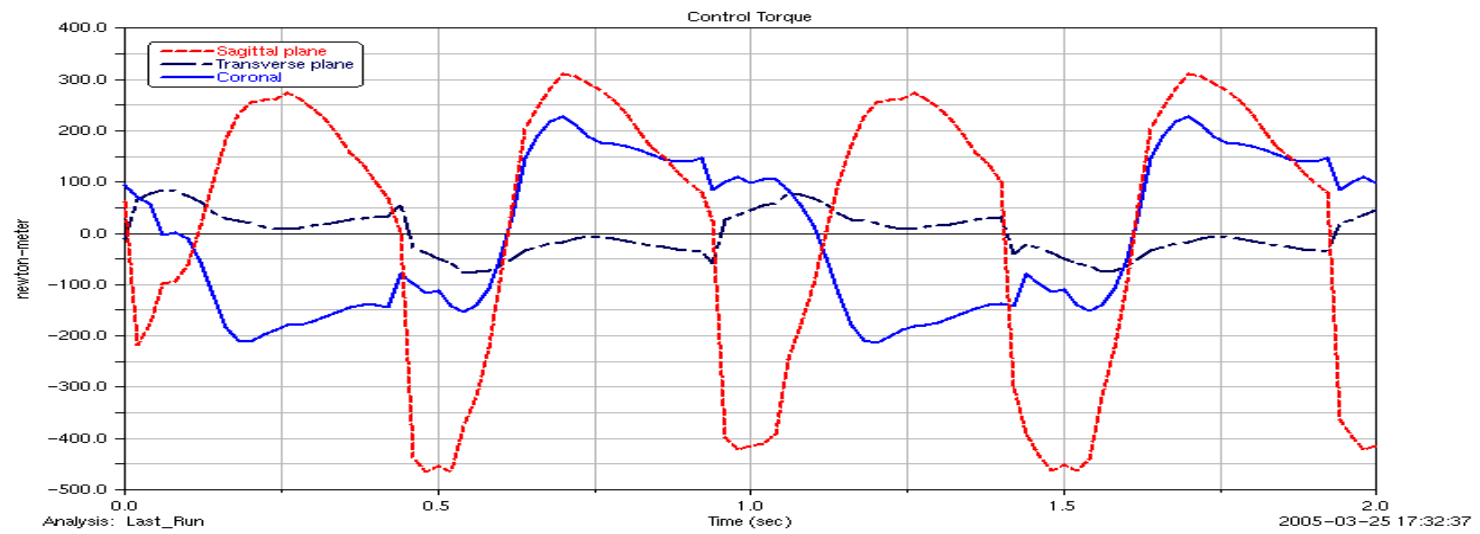

Figure (6 - 22) Control torque in virtual model 


\subsection{Physical Realization of Foot-release Mechanism}

To save fund and time, only one part of the entire model was going to be made. It was expected that the chosen part when added to the current PGO prototype can show the improvement of the function of current PGO. This can be answered through the validation experiment. If it does, it will give a strong hint that foot release PGO will be more effective than the current PGO even without experimental comparison on both physical models. It may also show that the simulation results are reliable and respectable. Therefore, foot release mechanism was chosen to be made into a prototype. With little modification, the current PGO can be ready for experiment. The prototype of foot release is shown in Figure $(6-23)$.



Figure (6 - 23) Prototype of foot release

\subsection{Step motor and Control}

Step motor AM23-150-2 is used to control foot release to ensure the mechanism extend to its maximum position in stance phase and retract to minimum position in swing 
phase. When using open loop control, there was no feed back. Just count how many revolutions or steps for motor when lowerplate moves from minimum position to maximum position. After start up, it will stop by itself after it rotates the exact steps set in. It is very easy to adjust the original position of the footplate.Just step on it and turn the step motor on then it will move under the weight and stay on the lowest position. It can not move against the weight. That makes sure motor always starts at the right position. A standalone controller DAX was used, which made the operation as simple as pushing a button. More details on control are included in the following discussion.

Because the step motor is already wired in parallel, it is ready for use. Just connect it to the controller. To work in stand alone, two things need to be set up first: storing code and setting operating button.

1. Code set-up:

- According to the manual, correctly set up hardware and connect controller to COM1 of the PC

- Set up communication between computer and controller through software EASI

- Enter a very short code started with P0, then R4000, then P0 end the code

2. Operating button set-up:

From the auxiliary I.O. connector, four pins for button operation are as:

P15 GND Power Common Ground

P17 Go Optically Isolated Input

P19 +5V Voltage Supply Output and

P20 Opto Supply Opto-Isolator Power-in.

- Connecting P19 and P20 to obtain power supply for P17 
- Put a momentary button between P17 and P15 to obtain "GO" input function. Each time the "GO" button is pushed, the controller will execute the code stored from address location 0 .

\subsection{Summary}

A foot release PGO virtual model is developed. The strength of each key part is checked and final functions are visualized through simulation. Foot release mechanism is made into a prototype. 


\section{VALIDATION OF THE MECHANISM AND DESIGN OF FOOT-RELEASE}

Because only one foot-release mechanism is made, it is hard to validate the whole system. However, the mechanism and design of the foot-release can be validated by adding the foot-release mechanism on the current PGO. It is necessary to know if the foot release is a right approach and if the design can satisfy the need of the approach. Therefore some experiments on this physical prototype are carried out to verify function. Before the experiments, the current PGO with foot release will be simulated to show the functional improvement of the current PGO. The results from the simulation can guide experiments, and in return the experiments can validate the simulation.

\subsection{Simulation of Current PGO with Foot-release}

\subsubsection{Simulation}

To show the improvement of current PGO with foot release, it is necessary to simulate it first then compare the results with those in Chapter 4. Because of addition of foot release the model may be changed slightly. For instance, there may be changes in position of COM and leg length of the model. That may make some error in comparison. However, the PGO can be simulated with foot release mechanism on but no release movement. Theoretically there is no error in the model. Simulation of current PGO with foot release movement is shown in Figure (7 - 1), and that simulation without release movement is shown in Figure $(7$ - 2). There is no significant difference between these figures, but the power consumption is different. The detailed comparison follows. 


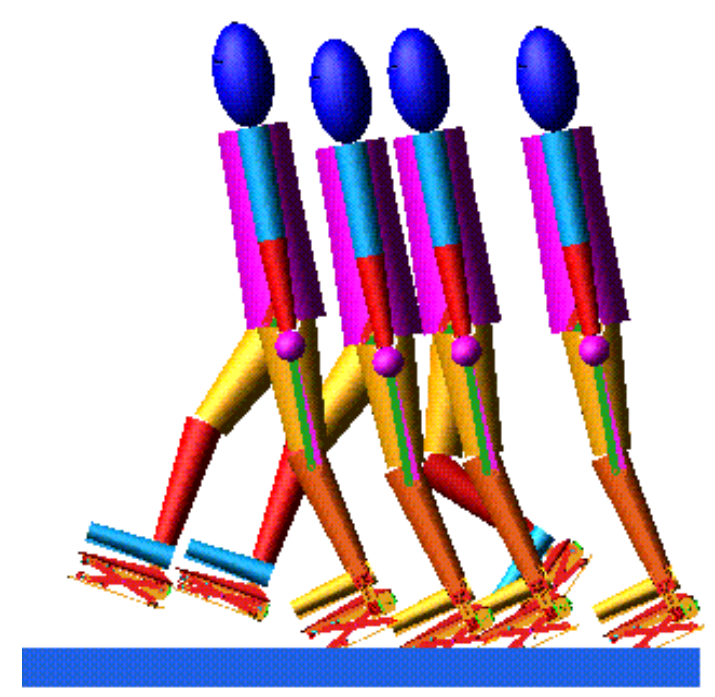

Figure (7 - 1) Simulation of current PGO with foot release movement

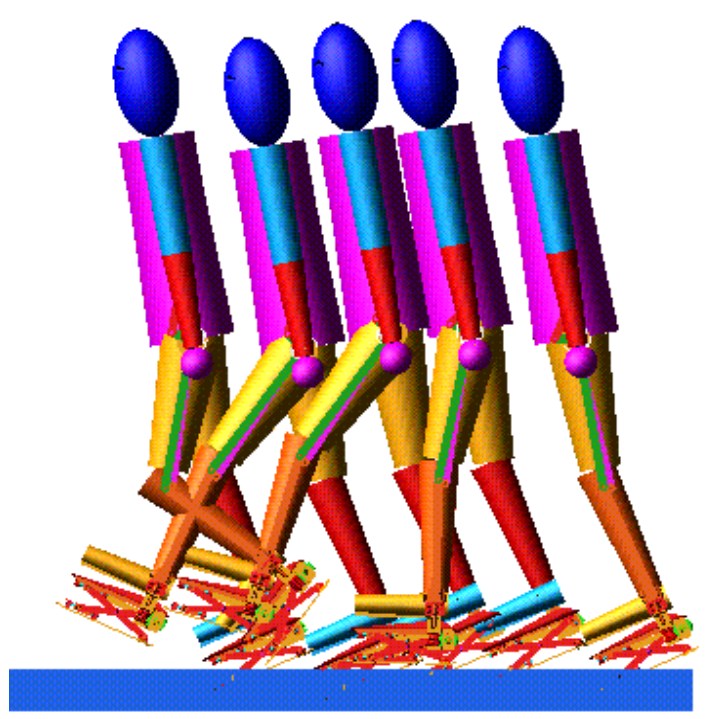

Figure (7 - 2) Simulation of current PGO without release movement

\subsubsection{Ground Reaction Forces}

Ground reaction forces are shown in Figure $(7-3)$ and Table 7 - 1 on page 128. There is no big difference after first cycle. The third peak in the curve of impact force with 
release movement is not supposed to exist. After the lower footplate retracts back, it will not contact with ground any more. Then why does the contact still happen? The reason is that the trailing leg is pulled forward faster than the release movement. Because the foot can not penetrate into the ground in real walking, this will not happen. Only after the release movement can the leg move forward. That will slow down the walking speed. To make simulation more accurate, it is necessary to know the real walking speed.

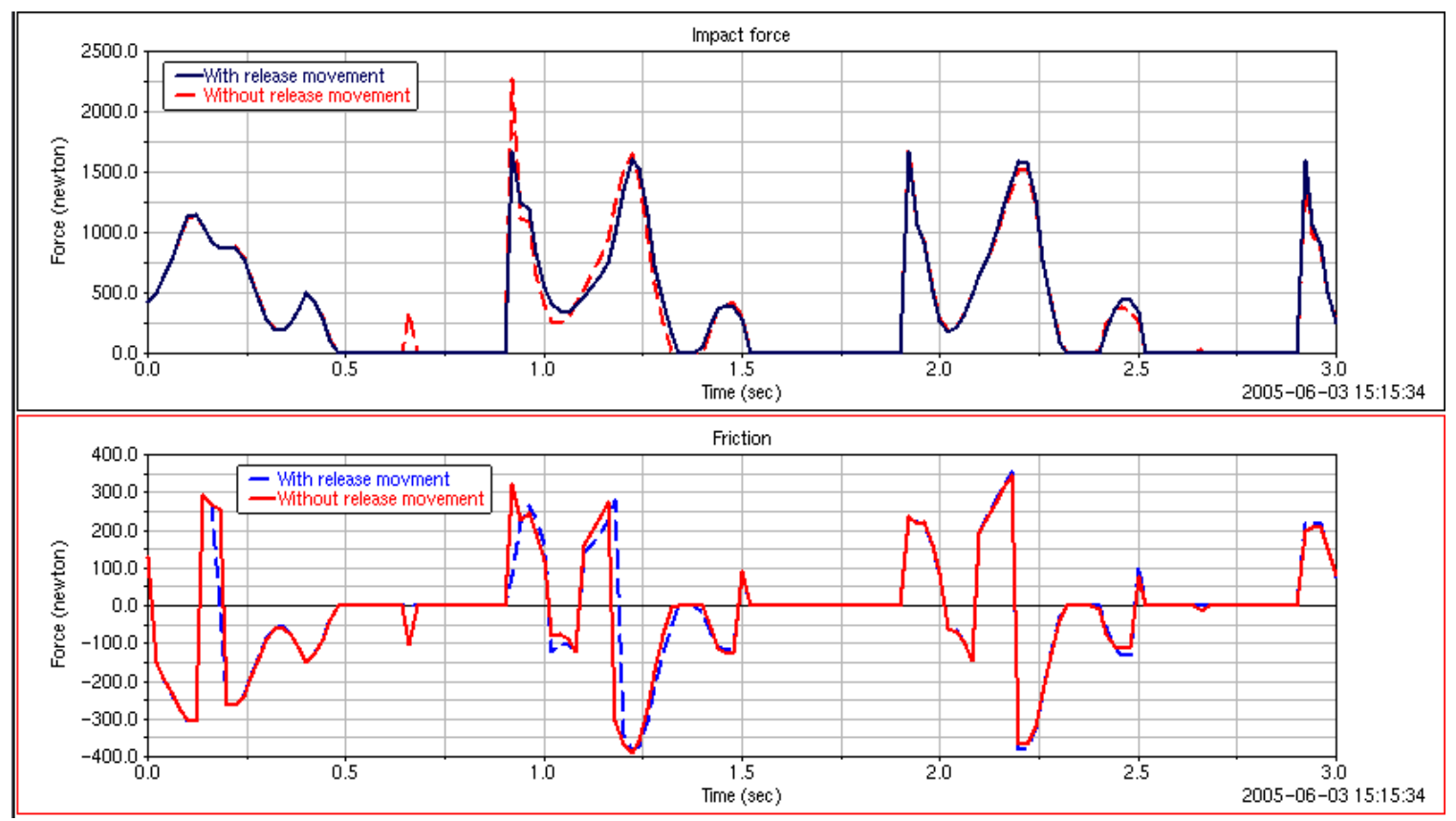

Figure (7 - 3) Comparison of ground reaction forces

Table(7 - 1): Comparison of Ground Reaction Forces

\begin{tabular}{c|c|c|c|c}
\hline & $\begin{array}{c}\text { Maximum } \\
\text { Force (N) }\end{array}$ & $\begin{array}{c}\text { Average } \\
(\mathrm{N})\end{array}$ & $\begin{array}{c}\text { Stance Phase } \\
(\% \text { of cycle })\end{array}$ & $\begin{array}{c}\text { Double } \\
\text { support }(\% \text { of } \\
\text { cycle })\end{array}$ \\
\hline $\begin{array}{c}\text { With release } \\
\text { movement }\end{array}$ & 1665.9 & 371.95 & 62 & 12 \\
\hline $\begin{array}{c}\text { Without release } \\
\text { movement }\end{array}$ & 2264.6 & 370.96 & 62 & 12 \\
\hline
\end{tabular}




\subsubsection{Moment and Power Consumption}

Because there was no big difference in ground reaction forces, the other parameters only changed slightly. However, the improvement can still be seen through the comparison of power consumption. The moment and power consumption are in Table $7-2$ on page 129 and Figure (7 - 4).

Table(7 - 2): Moment and Power for Current PGO with Foot Release

\begin{tabular}{c|c|c|c|c}
\hline \multirow{2}{*}{} & \multicolumn{2}{|c|}{ Moment (Nm) } & \multicolumn{2}{c}{ Power (W) } \\
\cline { 2 - 5 } & Max & Ave & Max & Ave \\
\hline $\begin{array}{c}\text { With release move- } \\
\text { ment }\end{array}$ & 192.4 & 40.36 & 2825 & 116.2758 \\
\hline $\begin{array}{c}\text { Without release } \\
\text { movement }\end{array}$ & 199.3 & 41.06 & 3555 & 130.5053 \\
\hline
\end{tabular}

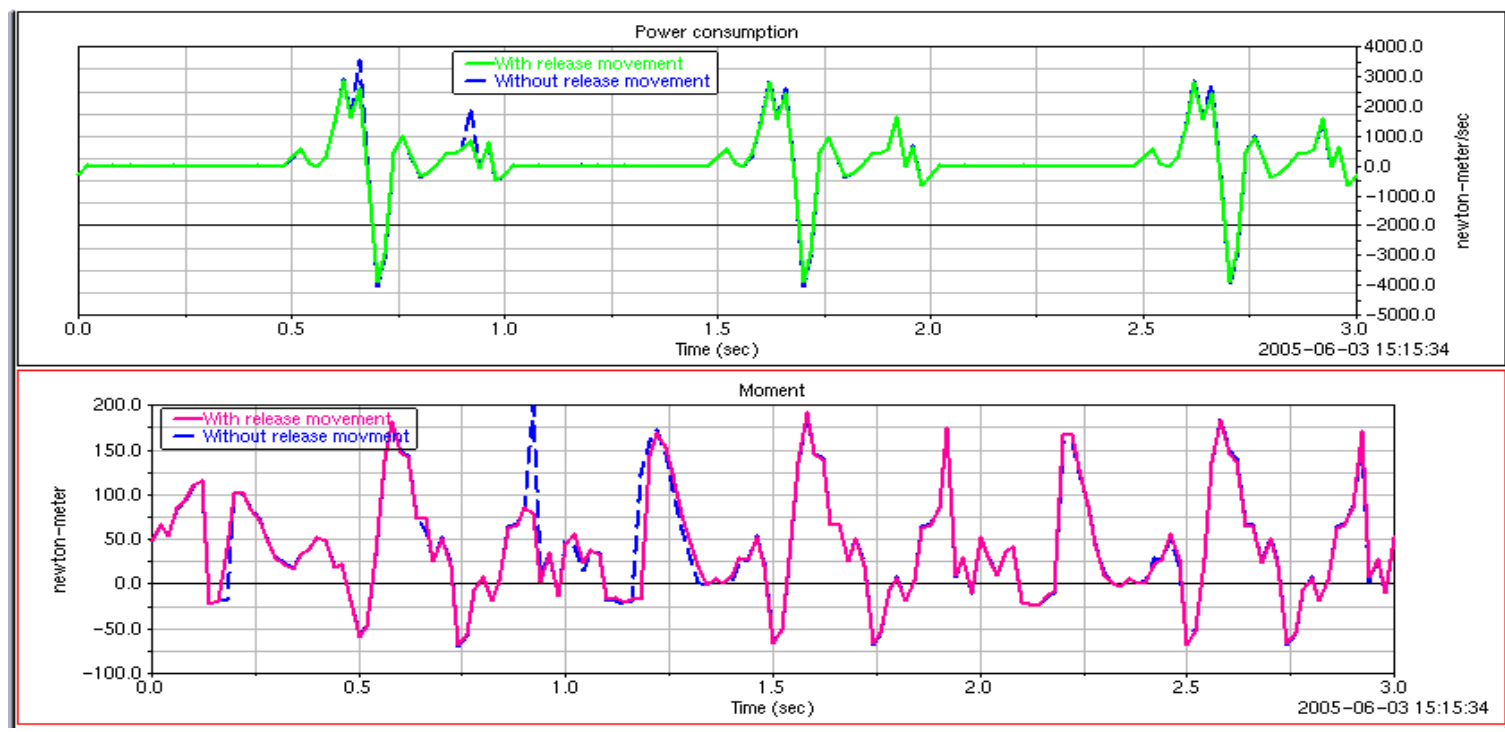

Figure (7 - 4) Comparison of moment and power consumption 


\subsubsection{Power Ratio}

The distances are shown in Figure (7 - 5). The power ratios are shown in Table 7 - 3 on page 130 . From the table it can seen that with release movement the power ratio decreases $9 \%$. The power ratio here can not be used to compare with those in chapter 5 . Here the simulation periods are 3 seconds. It can be simulated in 2 seconds, but simulation shows the curves before 2 seconds are not very stable. That may produce large error.

Table(7 - 3): Power Ratio of Current PGO with or Without Release Movement

\begin{tabular}{c|c|c}
\hline & $\begin{array}{c}\text { Distance in 3 sec. } \\
(\mathrm{m})\end{array}$ & $\begin{array}{c}\text { Power ratio } \\
(\mathrm{W} / \mathrm{m})\end{array}$ \\
\hline $\begin{array}{c}\text { With release move- } \\
\text { ment }\end{array}$ & .8878 & 261.94 \\
\hline $\begin{array}{c}\text { Without release } \\
\text { movement }\end{array}$ & .9092 & 287.08 \\
\hline
\end{tabular}

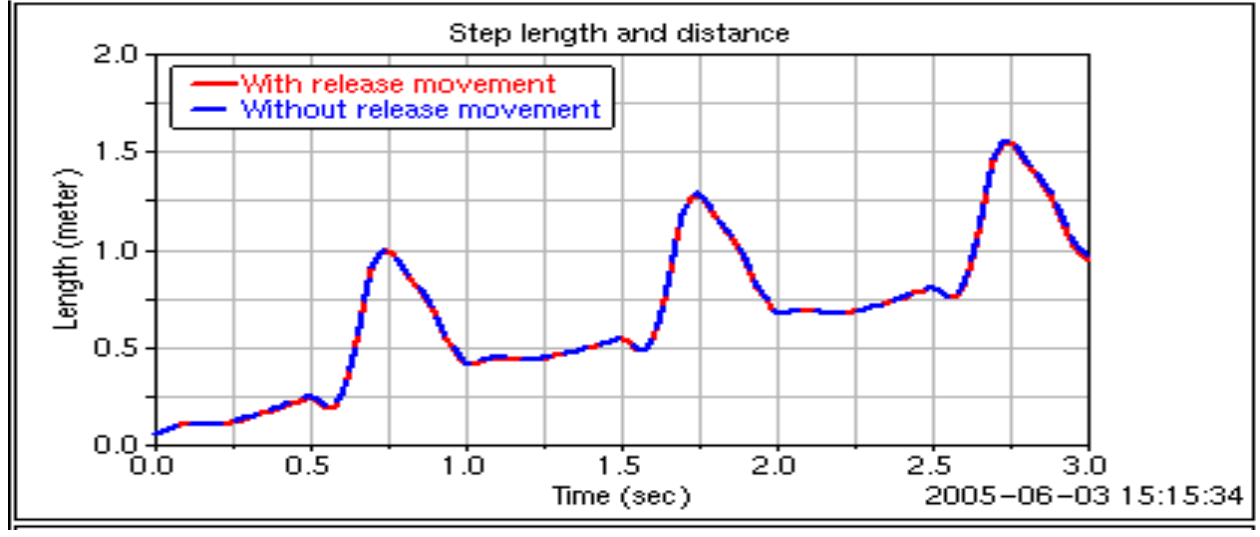

Figure (7 - 5) Comparison of walking distance with or without release 


\subsubsection{Control Torques}

Control torques in three planes are shown in Figure $(7-6)$ and Table 7 - 4 on page 131. No significant differences are seen.

Table(7 - 4): Control Torque of Current PGO With or Without Release Movement

\begin{tabular}{c|c|c|c|c|c|c}
\hline \multirow{2}{*}{} & \multicolumn{2}{|c|}{ Maximum (Nm) } & \multicolumn{2}{c|}{ Minimum (Nm) } & \multicolumn{2}{c}{ Average (Nm) } \\
\cline { 2 - 7 } & With & Without & With & Without & With & Without \\
\hline Sagittal & 436.87 & 435.7 & -961.4 & 964.47 & -139.55 & -138 \\
\hline Coronal & 416.99 & 389 & -315.7 & -338 & 3.12 & 2.3 \\
\hline Transverse & 155.94 & 158.56 & -165.2 & -168.9 & -4.05 & -3.85 \\
\hline
\end{tabular}

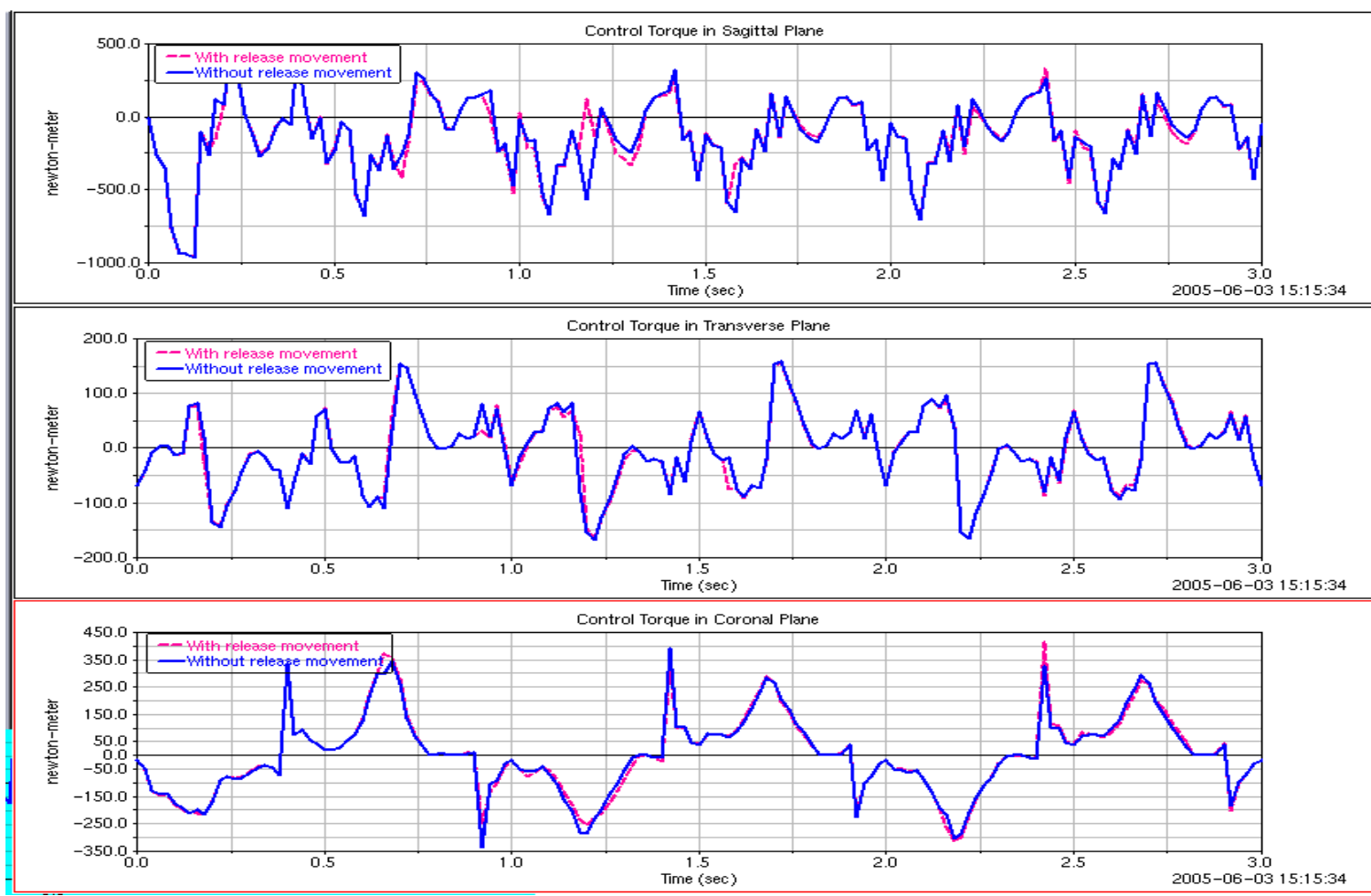

Figure (7 - 6) Comparison of control torque with or without release 


\subsection{Testing System Set up}

From Chapter 4 it was known the main cause of poor function is one leg moving at a time, which results in short steps and no clearance. Short step should be improved with new hip joints that allow both legs to move at the same time. Validation on this will be done when a physical model is built. Clearance can be improved by adding the foot release. The improvement will be validated as follows. Foot and ground contacting force of the moving leg can tell if the foot touches the ground. Thus, the footplate is used to test the contact force of the moving foot in both conditions, both with foot release movement and without it.

From Chapter 4 and video tape on current PGO it was seen that there was no clearance for trailing leg after toe off. Therefore, the testing system here is set up to test the ground reaction force of the trailing leg. The maximum extension angle of normal walking for hip is $10^{\circ}$. Thus the angle of trailing leg is adjustable from $0^{\circ}$ to $10^{\circ}$. Because the weight is usually supported by stance leg it is not needed to add force on the swing leg, only fasten the hip position.

The final setup of the testing system is shown in Figure (7 - 7). Two Siglab blocks or 8 Channels are used in this test because the Force plate has 8 outputs, which are Z1, Z2, Z3, $\mathrm{Z} 4, \mathrm{X}_{1+2}, \mathrm{X}_{3+4}, \mathrm{Y}_{1+4}$ and $\mathrm{Y}_{2+3}$. Then the impact force is $\mathrm{Z} 1+\mathrm{Z} 2+\mathrm{Z} 3+\mathrm{Z} 4$, the friction is $\sqrt{\left(X_{1+2}+X_{3+4}\right)^{2}+\left(Y_{1+4}+Y_{2+3}\right)}$.

The calibration is done by putting a known weight on the force plate. It is assumed there is no friction force under there, only vertical force. Then the calibration coefficient is the weight divided by mean value of the sum $\mathrm{Z1}, \mathrm{Z2}, \mathrm{Z3}$ and $\mathrm{Z} 4$. 


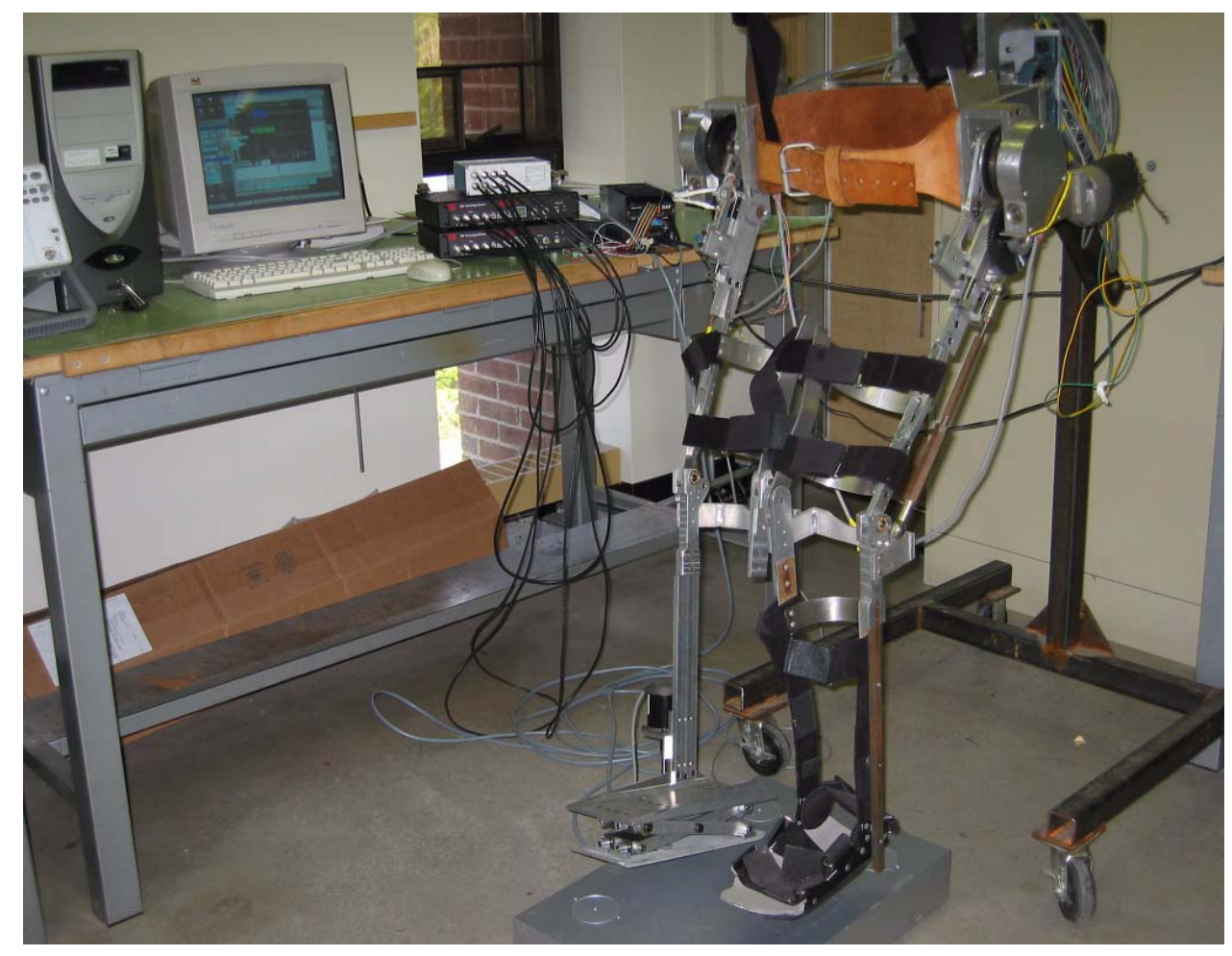

Figure (7 - 7) Testing system of ground reaction force for trailing leg

\subsection{Measured Results}

\subsubsection{Ground Contact Force With No Extension of Swing Leg}

In the condition of no extension of the swing leg the test is to check the forces starting from standing position. For normal walking the flexion of ankle will help to start from standing position. However in PGO the ankle is fixed. This may cause some friction on swing leg. The test data in Figure (7 - 8) shows it did cause impact force and friction climbing above the preload, while with release movement the force goes down below the preload and as does the friction. That means without ankle flexion the foot release will 
help to start from standing. In fact, the current PGO walking always walks from standing for every step because of only one leg moving at a time.
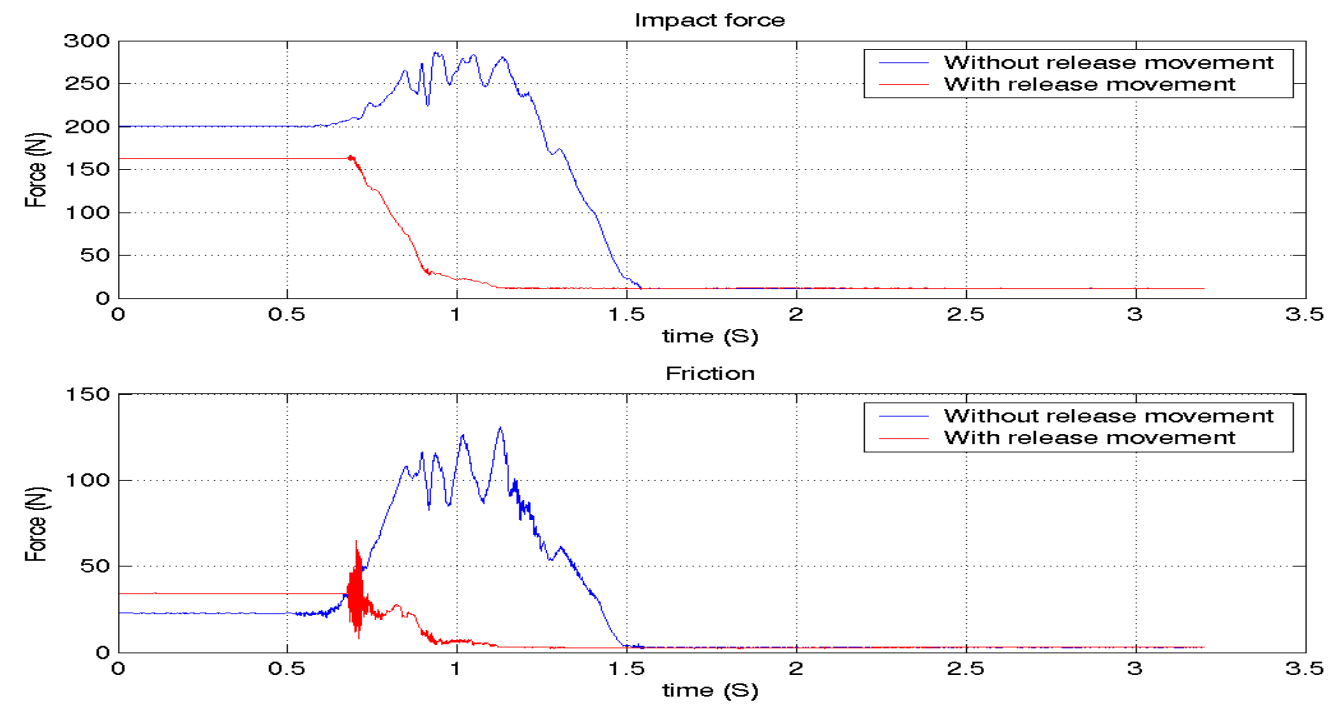

Figure (7 - 8) Measured ground reaction force with no extension

\subsubsection{Ground Contact Force With Five Degree Extension}

In this condition the clearance at the toe off of the swing leg is interested. The data is shown in Figure (7 - 9). Without release movement the impact force also climbs above the preload and results in big friction. That means while swing at this angle the clearance is decreasing. In fact the leg can not penetrate into the ground, the patient must lift his hip up to make clearance. With release movement the impact force goes down below the preload. That means the release mechanism can make clearance for the swing leg. It can be seen that the release mechanism can work under preload even though the power is very small. 

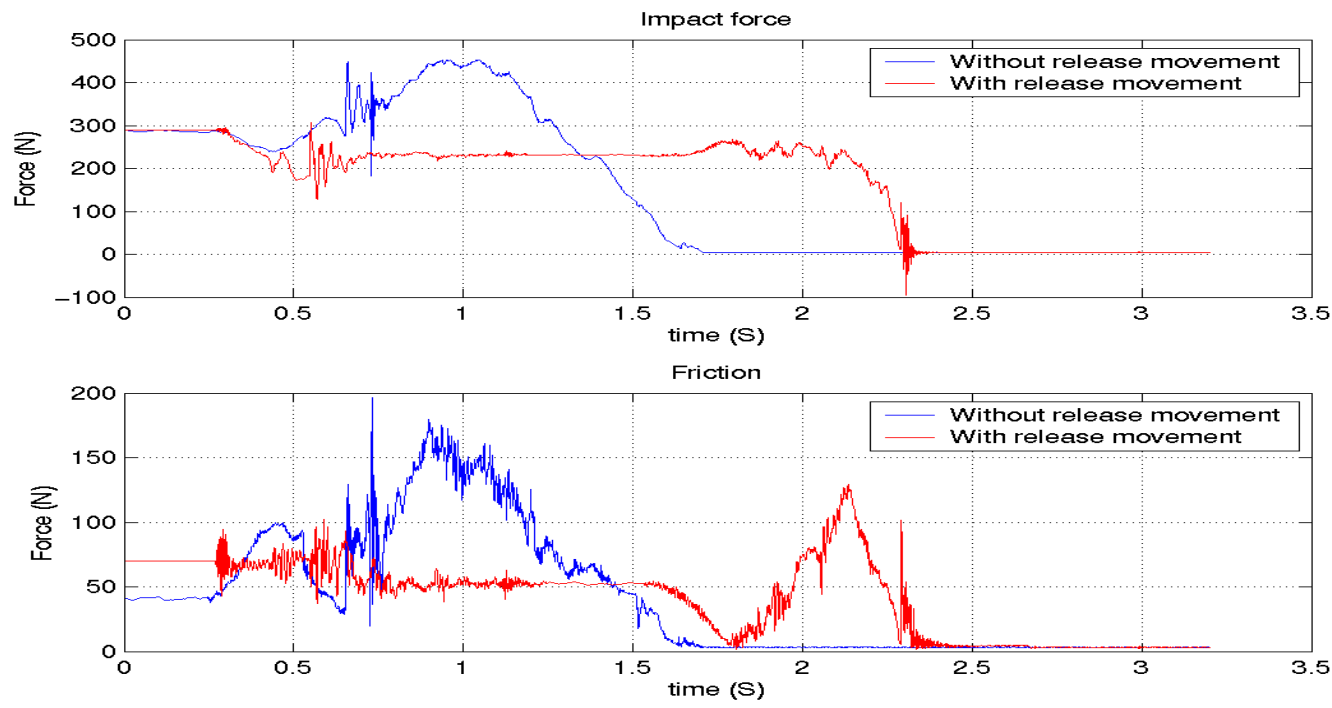

Figure (7 - 9) Measured ground reaction force with $5^{\circ}$ extension

\subsection{Discussion}

1. Difference between measurement and simulation. The third peak of the curve with release movement shown in Figure (7 - 3) means there is still penetration. However, the measured data in Figure (7 - 9) shows that with the release movement the impact force should be smaller than preload. If the preload is zero, there should have no force after the release movement. The third peak of Figure $(7-3)$ results from the penetration of the standing leg and relatively slower release movement. These two are the main reasons to shorten the clearance of the swing leg.

2. Mechanism of the foot release. The measured results in Figure (7 - 8) and Figure (7 9) show that at least between $0^{\circ}$ to $5^{\circ}$ of extension of the swing leg foot release causes impact force go down below the preload. That means it can make clearance if the pre- 
load is zero at the toe off. That also means that the release mechanism can provide some compensation for the locked knee and ankle.

3. Design of the foot release. The power consumption is very low for the mechanism. It can self lock at any position. It can work under the preload but not against the preload. and the release distance is about $30 \mathrm{~mm}$.

\subsection{Summary}

In this chapter both simulation and measurement validated the mechanism and the design of foot release. 


\section{CONCLUSIONS AND RECOMMENDATIONS}

\subsection{Summary and Conclusions}

In the course of this study, a model based experimental investigative approach was developed. First, a human model with 16 DOF was created and simulated. Foot ground contact model and upper body balance control strategy were validated by comparison of simulated results and measured data from Winter's book. Then they were adopted through this thesis. Real causes of poor function of current PGO were explored through the simulation of PGO walking model, and a trade-off between energy consumption, control complexity were found through the simulation of PGO mechanism model. Finally the foot release PGO virtual model was developed and a foot release was made into a prototype. The mechanism and design of foot release were validated through the experiment on current PGO with foot release. The following conclusions can be drawn from this research:

- Human walking model with 16 DOF was accurate enough for the investigation by comparison of simulated results and measured ones.

- Foot ground impact model was reliable and validated by measured impact forces from Winter's book.

- Foot ground friction model had some error, but total sliding was zero.

- Upper body balance control strategy was very simple and effective.

- The real causes of poor function of current PGO were found through the simulation of current PGO. The direct cause of poor function of current PGO was one leg moving at a time which resulted short steps and no clearance after toe off. The 
mechanical reason was no power working on most of stance phase to move the COM forward.

- PGO must be reciprocal.

- Power ratio in order were: Current PGO, current PGO with foot release, foot release, foot knee release, knee release and normal walking.

- Walking in the knee release PGO looks more natural and needs less power. However, if the knee and the hip are coupled, there is no standing position. If they are decoupled, the control system for knee is very complex.

- Foot release PGO is the trade off between energy consumption, standing position and control strategy. The foot release PGO can only replicate hip motion. However, the foot release mechanism is easier to develop than knee control system, and the user can get into a standing position with the foot release PGO.

- The foot release PGO has more advantage than RGO.

- Foot release mechanism is validated to be effective and can provide some compensation for the locked knee and ankle.

- Model based experimental investigation is very effective. It can reduce the cost of prototyping. Also, before the model is prototyped, most of functions are already known.

\subsection{Contributions}

Compared with previous work by others this thesis has produced several unique contributions, including: 
- Model based experimental investigation approach. There are a lot of simulation in human walking and a lot of simulation on robots in Chapter 1. This is the first time that combines both approaches.

- Foot-ground contact model with varied viscoelasticity. This model requires much more work. However, it makes the simulated results closer to the measured ones

- Upper body balance control strategy with two parallel primitive joints. This makes control simple. Trying out coefficient for Eqn (3-5) is not needed.

- Foot release mechanism. This mechanism is self locked by worm gear and controlled by step motor.

This approach can be applied to other areas. The models here can be used to investigate design and function of shoes, moment and power consumption for robot design.

\subsection{Recommendations for Future Work}

It can be seen that the whole physical model is not developed. Therefore there is no way to walk a patient in it. Some thing unforeseen even with the simulation may happen. Thus, the following recommendations for future work in this area are put forward:

1. Make the whole prototype.

2. Experiment on a real patient walking in it. Then it can be seen that how effective it is to restore the patient's walking ability.

3. Theoretically there is a lot of ways to improve the simulation:

- A new friction model needs to explore to get more accurate friction force. A clue for this was put forward in Chapter 3. 
- Upper body balance control can be replaced by whatever the patients use to keep their balance like walker or cane. That will make the simulation more accurate.

- Decrease the penetration. Penetration on stance leg will affect the clearance of the swing leg. This clearance does have effect on moment and power consumption.

- Speed the release movement. From the experiment there was no contact after release movement. While in the simulation the contact was still there.It is smaller than that with no release movement. That was the result of slower release movement relative to leg movement.

- The accuracy of simulation of PGO walking will be improved if a real PGO walking speed is used. In this paper, the hip joint speeds of PGO walking are the same as those of human walking.

- If the data for RGO walking is available, it can be simulated to compare energy consumption between RGO walking and foot release PGO walking. 


\section{REFERENCE}

[Anderson 1999] Anderson, F.C, Pandy, M.G., A dynamic optimization solution for vertical jumping in three dimensions, Computer Methods in Biomechanics and Biomedical Engineering, Vol.2, pp.201-231, 1999.

[Anderson 2001a] Anderson, F.C., Pandy, M.G., Static and dynamic optimization solutions for gait are practically equivalent, Journal of Biomechanics 34, 153-161, 2001.

[Anderson 2001b] Anderson,F.C., Pandy, M.G., Dynamic Optimization of Human Walking, ASME Journal of Biomechanical Engineering, Vol. 123, 381-390, Oct. 2001.

[Anderson 2002] Anderson, F.C., Pandy, M.G., Individual muscle contributions to support in normal walking, Gait and Posture 00 (2002) 1 -11.

[Amirouche 1990] Amirouche, F.M.L., Ider, S.K., Trimble, J., Analytical method for the analysis and simulation of human locomotion, ASME J. of Biomech. Eng., Vol. 112, pp. 379-386, Nov. 1990.

[Bernardi 1995] Bernardi, M., Canale, I., Castellano, V., Filippo, L. Di, Felici, F, Marchetti, M., The efficiency of walking of paraplegic patients using a reciprocating gait orthosis, Paraplegia, Vol. 33, pp.409-415, 1995.

[Bruderlin 1988] Bruderlin, A. W., Goal-directed, dynamic animation of bipedal locomotion, Master thesis, Simon Fraser University, 1988.

[Bruderlin 1989] Bruderlin, A. W., Calvert, T.W., Goal-directed, dynamic animation of human walking, Computer Graphics, v23, No. 3, pp.233-242, 1989.

[Bruneau 1999] Bruneau, O., Ouezdou, FB, Distributed ground/walking robot interaction, Robotica, vol.17, No. 3, pp 313-323, 1999. 
[Chow 1971] Chow, C.K., Jacobson, D.H., Studies of human locomotion via optimal programming, Mathematical Biosciences, Vol.10, pp. 239-306, 1971

[Colombo 2000] Colombo,G., Joerg, M., Schreier, R., Dietz, V., Treadmill training of paraplegic patients with a robotic orthosis, Journal of Rehabilitation Research and Development, Vol. 37, No. 6, pp.693-700, 2000.

[Colombo 2001] Colombo,G., Wirz, M., Dietz, V., Driven gait orthosis for improvement of locomotor training in paraplegic patients, Spinal Cord, v39, pp252-255, 2001.

[Douglas 1983] Douglas, R., Larson, PF, D’Ambrosia, R, McCall, RE, The LSU reciprocation gait orthosis, Orthopedics, v6, pp834-838, 1983.

[Downes 1994] Downes, C.G., Hill, S.L, Gray, J.O., Distributed control of an electrically powered hip orthosis. International Conference on Control '94, 21-24 March, pp. 24-30. [Englbrecht 2001] Englbrecht, M, Model-based experimental investigations on crutchwalking, Diploma Thesis, Lehrstuhl für Steuerungs- und Regelungstechnik, Technischen Universität München, Giugno 2001.

[Gerritsen 1995] Gerritsen, KGM, van den Bogert, AJ, Nigg, BM, Direct dynamics simulation of the impact phase in heel-toe running, J. Biomechanics, v28, No.6, pp661-668, 1995.

[Gilchrist 1996] Gilchrist, LA, Winter, DA, A two-part, viscoelastic foot model for use in gait simulations, J. Biomechanics, v29, No. 6, pp795-798, 1996.

[Gilchrist 1997] Gilchrist, LA, Winter, DA, A multisegment computer simulation of normal human gait, IEEE Transactions on Rehabilitation Engineering, v5, No. 4, 1997. [Hanavan 1964] 
[Harkema 2001] Harkema, SJ, Neural plasticity after human spinal cord injury: application of locomotor training to the rehabilitation of walking, The Neuroscientist, v7, No. 5, pp455-468, 2001.

[Hase 2002a] Hase, K., Yamazaki, N., Computer simulation study of human locomotion with a three-dimensional entire-body neuro-musculo-skeletal model (I. Acquisition of normal walking), JSME International Journal, Vol. 45, No. 4, pp.1040-1050, 2002. [Hase 2002b] Hase, K., Obinata, G., Computer simulation study of human locomotion with a three-dimensional entire-body neuro-musculo-skeletal model (II. Biomechanical relationship between walking stability and neuro-musculo-skeletal system), JSME International Journal, Vol. 45, No. 4, pp.1051-1057, 2002.

[Hase 2002c] Hase, K., Obuchi, S., Computer simulation study of human locomotion with a three-dimensional entire-body neuro-musculo-skeletal model (III. Simulation of pathological walking and its application to rehabilitation engineering), JSME International Journal, Vol. 45, No. 4, pp.1058-1064, 2002.

[Hase 2002d] Hase, K., Yokoi, T., Computer simulation study of human locomotion with a three-dimensional entire-body neuro-musculo-skeletal model (IV. Simulation of running motion and its transition process), JSME International Journal, Vol. 45, No. 4, pp.10651072, 2002.

[Hesse 2000] Hesse, S., Uhlenbrock,D., A mechanized gait trainer for restoration of gait, Journal of Rehabilitation Research and Development Vol. 37 No. 6, pp. 701-708, 2000. [Hirai 1997] Hirai, K, Current and future perspective of Honda humanoid robot, IEEE/ RSJ International Conference on Intelligent Robots and Systems, pp500-508, 1997. 
[Hirai 1998] Hirai, K, Hirose, M, Haikawa, Y, Takenaka, T, The development of Honda humanoid robot, Proceedings of the 1998 IEEE International Conference on Robotics \& Automation, pp1321-1326, 1998.

[Hirata 2002] Hirata, R., Sakaki, T., Okada, S., Nakamoto, Z., Hiraki, N., Okajima, Y., Uchida, S., Tomita, Y., Horiuchi, T., BRMS: Bio-resposive motion system (Rehabilitation system for stroke patients), IEEE International Conference on Intelligent Robots and Systems, v 2, p 1344-1348, 2002.

[Inman 1981] Inman, V.T., Raltson, H.J., Todd, F., Human walking, Baltimore: Williams \& Wilkins, 1981.

[Jefferson 1990] Jefferson, R.J., Whittle, M.W. Performance of three walking orthoses for the paralysed: a case study using gait analysis. Prosthetics and Orthotics International, 14, 103-110, 1990.

[Ju 1988] Ju, M, Mansour, J.M., Simulation of the double limb support phase of human gait, ASME J. of Biomech. Eng., Vol. 110, pp. 223-229, Aug. 1988.

[Kasaoka 2001] Kasaoka, K., Sankai, Y., Predictive control estimating operator's intention for stepping-up motion by exo-sckeleton type power assist system HAL, IEEE International Conference on Intelligent Robots and Systems, v 3, p 1578-1583, 2001. [Kawamoto 2002] Kawamoto, H., Sankai, Y., Comfortable power assist control method for walking aid by HAL-3, Proceedings of the IEEE International Conference on Systems, Man and Cybernetics, v 4, p 447-452, 2002.

[Ko 1994] Ko, H., Kinematic and dynamic techniques for analyzing, predicting, and animating human locomotion, Ph.D. Dissertation, University of Pennsylvania, 1994; 
[Koopman 1995] Koopman, B., Grootenboer, H.J., de Jongh, H.J., An inverse dynamics model for the analysis, reconstruction and prediction of bipedal walking, J. Biomechanics Vol.28, No.11, pp.1369-1376, 1995.

[Lee 2002a] Lee, S., Sankai, Y., Power assist control for leg with HAL-3 based on virtual torque and impedance adjustment, Proceedings of the IEEE International Conference on Systems, Man and Cybernetics, v 4, p 453-458, 2002.

[Lee 2002b] Lee, S., Sankai, Y., Power assist control for walking aid with HAL-3 based on EMG and impedance adjustment around knee joint, Proceedings of the 2002 IEEE/RSJ International Conference on Intelligent Robots and Systems, v 2, p1499-1504, 2002. [McGeer 1990] McGeer, T, Passive dynamic walking, Intern. J. Robot Res. 9(2), 68-82, Apr. 1990.

[McGeer 1993] McGeer, T, Dynamics and control of bipedal locomotion, J. Theor. Biol. 163, 277-314, 1993.

[Middleton 1998] Middleton, J.W. etal. A medial linkage orthosis to assist ambulation after spinal cord injury. Prosthetics and Orthotics International, 1998, 22, 258-264. [Neptune 2000] Neptune, RR, Wright, IC, Van Den Bogert, AJ, A method for numerical simulation of single limb ground contact events: application to heel-toe running, Computer Methods in Biomechanics and Biomedical Engineering, vol.3, pp321-334, 2000. [Neptune 2001] Neptune, R.R., Kautz, S.A., Zajac, F.E., Contributions of the individual ankle plantar exors to support, forward progression and swing initiation during walking, Journal of Biomechanics, v34, pp1387-1398, 2001.

[Onyshko 1980] Onyshko, S., Winter, D.A., A mathematical model for the dynamics of human locomotion, J. Biomechanics Vol. 13,pp. 361-368, 1980. 
[Ouezdou 1998] Ouezdou, FB, Bruneau, O., Guinot, JC, Dynamic analysis tool for legged robots, Multibody System Dynamics, v2, pp369-391, 1998.

[Pandy 1988a] Pandy, MG, Berme, N, A numerical method for simulating the dynamics of human walking, J. Biomechanics Vol. 21, No. 12, pp. 1043-1051, 1988.

[Pandy 1988b] Pandy, MG, Berme, N, Synthesis of human walking: A planar model for single support, J. Biomechanics Vol. 21, No. 12, pp. 1053-1060, 1988.

[Pandy 1989a] Pandy, MG, Berme, N, Quantitative assessment of gait determinants during single stance via a three-dimensional model - Part 1. Normal gait, J. Biomechanics Vol. 22, No. 6/7, pp. 717-724, 1989.

[Pandy 1989b] Pandy, MG, Berme, N, Quantitative assessment of gait determinants during single stance via a three-dimensional model - Part 2. Pathological gait, J. Biomechanics Vol. 22, No. 6/7, pp. 725-733, 1989.

[Pandy 2001] Pandy, M.G., Computer modeling and simulation of human movement, Annu. Rev. Biomed. Eng. 3:245-73, 2001.

[Rogers 1993] Rogers, D.S., Joint torque and powered requirements for a powered gait orthosis device, Thesis, Louisiana State University, 1993.

[Ruthenberg 1994] Ruthenberg, BJ, Force analysis, effect of varying time ratio, and a finite element optimization of the powered gait orthosis, Thesis, Michigan Technological University, 1994.

[Ruthenberg 1997] Ruthenberg, BJ, Wasylewski, NA, Beard, JE. An experimental device for investigating the force and power requirements of a powered gait orthosis. Journal of Rehabilitation Research and Development, 34(2), 203-213, 1997. 
[Schmidt 2002a] Schmidt, H.; Sorowka, D.; Hesse, S.; Bernhardt, R., Design of a robotic walking simulator for neurological rehabilitation, IEEE International Conference on Intelligent Robots and Systems, v 2, p 1487-1492, 2002.

[Schmidt 2002b] Schmidt, H.; Sorowka, D.; Hesse, S.; Bernhardt, R., Robotic walking simulator for neurological gait rehabilitation, Annual International Conference of the IEEE Engineering in Medicine and Biology, v 3, p 2356-2357, 2002.

[Seireg 1989] Seireg, A., Arvikar, R. Biomechanical analysis of the musculoskeletal structure for medicine and sports, New York: Hemisphere Pub. Corp., c1989.

[Silva 2001] Silva, FM, Goal-oriented biped walking based on force interaction control, Proceedings of the IEEE International Conference on Robotics \& Automation, pp41224127, 2001.

[Stokes 1992] Stokes, A., Gear handbook, Butterworth-Heinemann Ltd, 1992.

[Taga 1998] Taga, G., A model of the neuro-musculo-skeletal system for anticipatory adjustment of human locomotion during obstacle avoidance, Biological Cybernetics, 78, pp.9-17, 1998.

[Taga 2000] Taga, G., Nonlinear dynamics of the human motor control -real-time and anticipatory adaptation of locomotion and development of movements, Proceedings of the International Symposium on Adaptive Motion of Animals and Machines, 2000.

[Tashman 1995] Tashman, S., Zajac, F.E., Perkash, I, Modeling and simulation of paraplegic ambulation in a reciprocating gait orthosis, ASME J. of Biomech. Eng., Vol.117, pp.300-308, 1995. 
[Van Den Bogert 1989] Van Den Bogert, AJ, Schamhardt, HC, Crowe, A., Simulation of quadrupedal locomotion using a rigid body model, J. Biomechanics, v22, No. 1, pp33-41, 1989.

[Whittle 1991] Whittle, M.W. etal. A Comparative Trial of Two Walking Systems for Paralysed People, Paraplegia, 29, 97-102, 1991.

[Winter 1991] Winter, D. A., The biomechanics and motor control of human gait: Normal, Elderly and Pathological, 2nd edition, University of Waterloo Press, 1991.

[Wojtyra 2000] Wojtyra, M., Dynamical analysis of human walking, Conference on Adams, 2000.

[Wright 1998] Wright, I.C., Neptune, R.R., van den Bogert, A.J., Nigg, B.M., Passive regulation of impact forces in heel-toe running, Clinical Biomechanics, Vol.13, pp. 521-531, 1998.

[Wright 2000] Wright, I.C., Neptune, R.R., van den Bogert, A.J., Nigg, B.M.,The effects of ankle compliance and flexibility on ankle sprains, Med. Sci. Sports Exerc., Vol. 32, No. 3, pp. 260-265, 2000.

[Yamaguchi 1990] Yamaguchi, G.T., and Zajac, F.E., Restoring unassisted natural gait to paraplegics via functional neuromuscular stimulation: a computer simulation study. IEEE Trans. Biomed. Eng., vol. 37:886-902, 1990.

[Yano 1997] Yano, H., Kaneko, S., Nakazawa, K., Yamamoto, S-I., Bettoh, A., A new concept of dynamic orthosis for paraplegia: the weight bearing control (WBC) orthosis, Prosthetics and Orthoics International, 21, pp. 222-228, 1997. 
[Zajac 2002] Zajac, FE, Neptune, RR, Kautz, SA, Biomechanics and muscle coordination of human walking Part I: introduction to concepts, power transfer, dynamics and simulations, Gait and Posture, v16, pp215-232, 2002.

[Zajac 2003] Zajac, FE, Neptune, RR, Kautz, SA, Biomechanics and muscle coordination of human walking Part II: lessons from dynamical simulations and clinical implications, Gait and Posture, v17, pp1-17, 2003.

[Hashimoto 2002] Hashimoto, S., etal, Humanoid robots in Waseda University - Hadaly-2 and WABIAN, Autonomous Robots, 12, 25-38, 2002.

[Popvic 1999] Popvic, D., etal, Optimal control of walking with functional electrical stimulation: a computer simulation study, IEEE Trans. On Rehab. Eng., Vol. 7, No. 1, 1999. [Popvic 2002] Popvic, D., Radulovic, M., Schwirtlich, L., Jaukovic, N., Automatic vs hand-controlled walking of paraplegics, Medical Engineering \& Physics, 2002. [Jonic 1999] Jonic, S., Jankovic, T., Gajic, V., Popvic, D., Three machine learning techniques for automatic determination of rules to control locomotion, IEEE Trans. on Biomed. Eng., Vol. 46, No.3, 1999.

[Kumar 1997] Kumar, V., Rahman, T., Krovi, V., Assistive Devices For People With Motor Disabilities, Wiley Encyclopaedia of Electrical and Electronics Engineering, 1997. [Lee 2001] Lee, C.Y., Seo, K.H., Oh, C., Lee, J.J., A System for Gait Rehabilitation with Body Weight Support: Mobile Manipulator Approach, International Journal of HumanFriendly Welfare Robotic Systems, Vol.2, No.3, PP.16-21, 2001. [Popvic 1993] Popvic, D., Schwirtlich, L., Design and evaluation of the self-fitting modular orthosis (SFMO), IEEE Trans. On Rehab. Eng., Vol. 1, No.3, 1993. 


\section{APPENDIX A}

\section{Ideas Models}

1. Normal walking model: healthyman.mf1, including healthyman and footreleasedpgo two assemblies.

2. Current PGO model: pgophysicmodel.mf1, including oldpgophysicmodel, oldpgowithfootrelease subassemblies.

3. PGO virtual model: PGOdesign.mf1. Main assembly is man_newPGO, including human, NewPGOphysicalmodel, NewPGOphysicalmodel_L subassembly which is also including foot_release_linear_bearing and PGOmodel (hip joint) subassembly.

\section{Adams Models}

1. Normal walking model: healthyman_distributed_force.bin

2. Current PGO model: oldphysicmodel_D.bin

3. PGO mechanism model: footreleasedpgo1_D.bin for knee release, footreleasedpgo2_D.bin for foot release, footreleasedpgo4_D.bin for foot \& knee release

4. PGO virtual model: man_newPGO_virtual.bin, foot_release_linear_bearing.bin for foot release, PGOmodel_1.bin for hip joint

5. Current PGO with foot release model: oldpgowithfootrelease.bin for release movement, oldpgowithfootrelease_norelease.bin for no release movement 


\section{APPENDIX B}

\section{Matlab Files}

\section{B.1 regression.m}

This code is compiled to fit the test data from Winter's book with Fourier series, then the data is approximated with a periodical function.

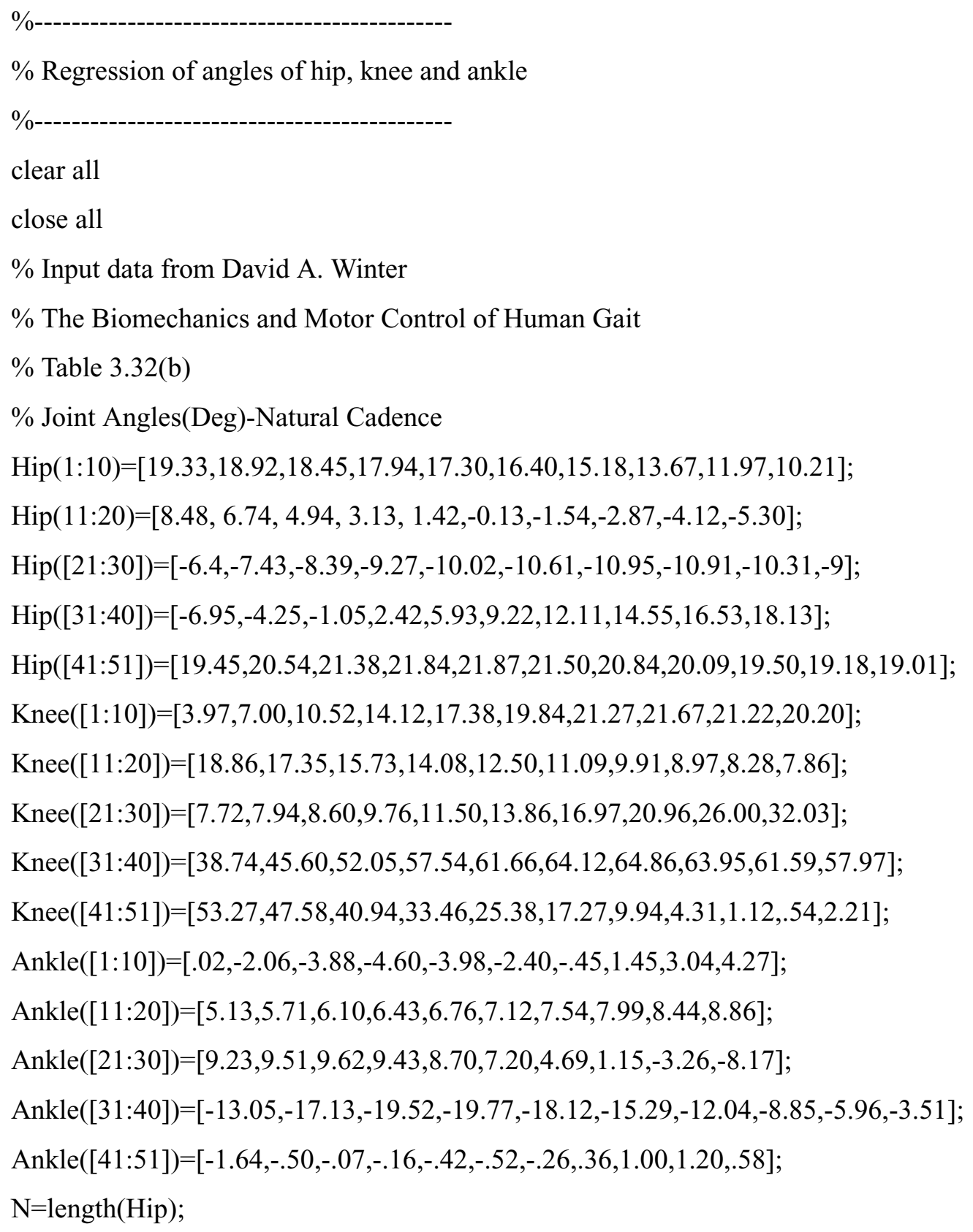




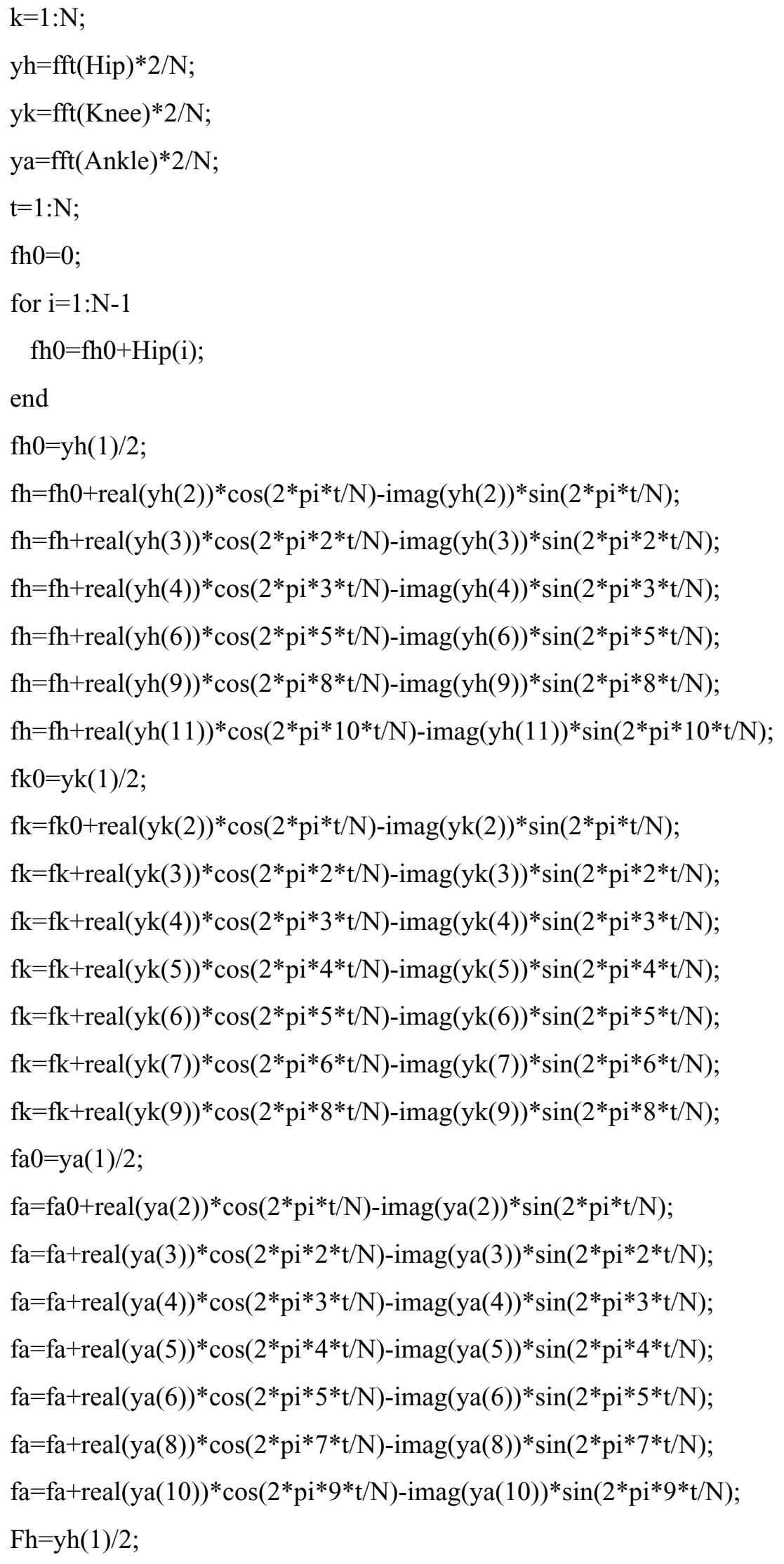


$\mathrm{Fk}=\mathrm{yk}(1) / 2$;

$\mathrm{Fa}=\mathrm{ya}(1) / 2$;

for $\mathrm{i}=1: \mathrm{N} / 2$

$$
\begin{aligned}
& \mathrm{Fh}=\mathrm{Fh}+\operatorname{real}(\mathrm{yh}(\mathrm{i}+1)) * \cos \left(2 * \mathrm{pi}^{*} *^{*} \mathrm{t} / \mathrm{N}\right)-\operatorname{imag}(\mathrm{yh}(\mathrm{i}+1)) * \sin \left(2 * \mathrm{pi}^{*}{ }_{\mathrm{i}} * \mathrm{t} / \mathrm{N}\right) \text {; } \\
& \mathrm{Fk}=\mathrm{Fk}+\mathrm{real}(\mathrm{yk}(\mathrm{i}+1)) * \cos \left(2 * \mathrm{pi}^{*} \mathrm{i} * \mathrm{t} / \mathrm{N}\right)-\operatorname{imag}(\mathrm{yk}(\mathrm{i}+1)) * \sin \left(2 * \mathrm{pi}^{*} \mathrm{i}^{*} \mathrm{t} / \mathrm{N}\right) \\
& \mathrm{Fa}=\mathrm{Fa}+\mathrm{real}(\mathrm{ya}(\mathrm{i}+1)) * \cos \left(2 * \mathrm{pi}^{*} \mathrm{i} * \mathrm{t} / \mathrm{N}\right)-\mathrm{imag}(\mathrm{ya}(\mathrm{i}+1)) * \sin \left(2 * \mathrm{pi}^{*} \mathrm{i} * \mathrm{t} / \mathrm{N}\right) \text {; }
\end{aligned}
$$

end

figure('position',[0 0600 600]);

grid on; hold on

plot(k-1,Hip,'r-'); hold on

plot(k-1,Knee,'k-');

plot(k-1,Ankle,'g-');

$\operatorname{plot}\left(\mathrm{t}-1, \mathrm{fh},{ }^{\prime} \mathrm{r}-\mathrm{-}^{\prime}\right)$;

plot(t-1,fk,'k--');

plot(t-1,fa,'g--');

plot(t-1,Fh,'b--');

plot(t-1,Fk,'c--');

$\operatorname{plot}\left(\mathrm{t}-1, \mathrm{Fa},{ }^{\prime} \mathrm{y}-{ }^{\prime}\right)$;

legend('Hip','Knee','Ankle');

figure('position',[50 50600 600]);

plot(k-1,abs(yh),'r-'); hold on

$\operatorname{plot}\left(\mathrm{k}-1, \mathrm{abs}(\mathrm{yk}),{ }^{\prime} \mathrm{k}-{ }^{\prime}\right)$;

plot(k-1,abs(ya),'g-');

$\left.\operatorname{disp}\left(' \mathrm{fh}=\mathrm{fh} 0+\operatorname{real}(\mathrm{yh}(2)) * \cos \left(2 * \mathrm{pi}^{*} \mathrm{t} / \mathrm{N}\right)-\operatorname{imag}(\mathrm{yh}(2)) * \sin \left(2 * \mathrm{pi}{ }^{*} \mathrm{t} / \mathrm{N}\right)\right)^{\prime}\right)$;

$\left.\operatorname{disp}\left(' \mathrm{fh}=\mathrm{fh}+\mathrm{real}(\mathrm{yh}(3)) * \cos \left(2 * \mathrm{pi}^{*} 2 * \mathrm{t} / \mathrm{N}\right)-\operatorname{imag}(\mathrm{yh}(3)) * \sin \left(2 * \mathrm{pi}^{*} 2 * \mathrm{t} / \mathrm{N}\right)\right)^{\prime}\right)$;

$\left.\operatorname{disp}(' \mathrm{fh}=\mathrm{fh}+\mathrm{real}(\mathrm{yh}(4)) * \cos (2 * \mathrm{pi} * 3 * \mathrm{t} / \mathrm{N})-\operatorname{imag}(\mathrm{yh}(4)) * \sin (2 * \mathrm{pi} * 3 * \mathrm{t} / \mathrm{N}))^{\prime}\right)$;

$\operatorname{disp}\left(' \mathrm{fh}=\mathrm{fh}+\mathrm{real}(\mathrm{yh}(6)) * \cos \left(2 * \mathrm{pi}^{*} 5 * \mathrm{t} / \mathrm{N}\right)-\operatorname{imag}(\mathrm{yh}(6)) * \sin \left(2 * \mathrm{pi}^{*}{ }^{*} * \mathrm{t} / \mathrm{N}\right){ }^{\prime}\right)$;

$\left.\operatorname{disp}(' \mathrm{fh}=\mathrm{fh}+\mathrm{real}(\mathrm{yh}(9)) * \cos (2 * \mathrm{pi} * 8 * \mathrm{t} / \mathrm{N})-\operatorname{imag}(\mathrm{yh}(9)) * \sin (2 * \mathrm{pi} * 8 * \mathrm{t} / \mathrm{N}))^{\prime}\right) ;$

$\operatorname{disp}\left(' \mathrm{fh}=\mathrm{fh}+\mathrm{real}(\mathrm{yh}(11)) * \cos \left(2 * \mathrm{pi}^{*} 10 * \mathrm{t} / \mathrm{N}\right)-\operatorname{imag}(\mathrm{yh}(11)) * \sin \left(2 * \mathrm{pi}^{*} 10 * \mathrm{t} / \mathrm{N}\right){ }^{\prime}\right)$;

fh0

$\% \operatorname{disp}(' y h(2)=') ; y h(2)$

$\operatorname{yh}(2)$

$\operatorname{disp}\left({ }^{\prime} y h(3)='\right) ; y h(3)$ 


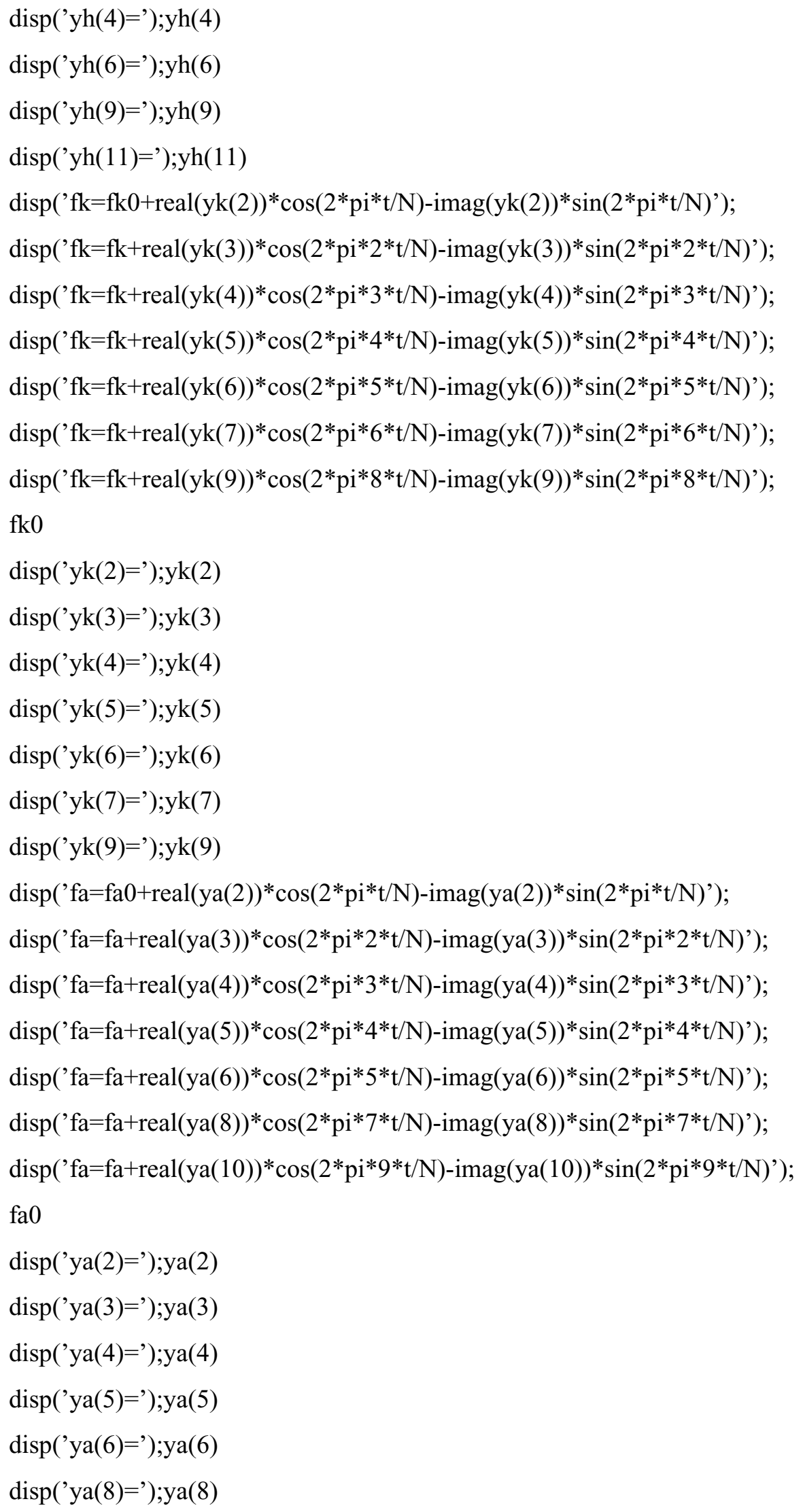


$\operatorname{disp}(' y a(10)=') ; y a(10)$

\%---------- Shift data for the other leg

RHip(1:26)=Hip(26:51);

$\operatorname{RHip}(27: 51)=\operatorname{Hip}(1: 25)$;

RKnee(1:26)=Knee(26:51);

RKnee(27:51)=Knee( $(1: 25)$;

RAnkle(1:26)=Ankle(26:51);

RAnkle(27:51)=Ankle(1:25);

Ryh $=\mathrm{fft}($ RHip) $* 2 / \mathrm{N}$;

$\mathrm{Ryk}=\mathrm{fft}(\mathrm{RKnee}) * 2 / \mathrm{N}$;

$\mathrm{Rya}=\mathrm{fft}(\mathrm{RAnkle}) * 2 / \mathrm{N}$;

$\mathrm{Rfh} 0=\operatorname{Ryh}(1) / 2$;

$\mathrm{Rfh}=\mathrm{Rfh} 0+\operatorname{real}(\operatorname{Ryh}(2)) * \cos \left(2 * \mathrm{pi}^{*} \mathrm{t} / \mathrm{N}\right)-\operatorname{imag}(\operatorname{Ryh}(2)) * \sin (2 * \mathrm{pi} * \mathrm{t} / \mathrm{N})$;

$\mathrm{Rfh}=\mathrm{Rfh}+\operatorname{real}(\operatorname{Ryh}(3)) * \cos (2 * \mathrm{pi} * 2 * \mathrm{t} / \mathrm{N})-\operatorname{imag}(\operatorname{Ryh}(3)) * \sin (2 * \mathrm{pi} * 2 * \mathrm{t} / \mathrm{N})$;

$\mathrm{Rfh}=\mathrm{Rfh}+\operatorname{real}(\operatorname{Ryh}(4)) * \cos \left(2 * \mathrm{pi}^{*} 3 * \mathrm{t} / \mathrm{N}\right)-\operatorname{imag}(\operatorname{Ryh}(4)) * \sin \left(2 * \mathrm{pi}^{*} 3 * \mathrm{t} / \mathrm{N}\right)$;

$\mathrm{Rfh}=\mathrm{Rfh}+\operatorname{real}(\operatorname{Ryh}(6)) * \cos \left(2 * \mathrm{pi}^{*} 5 * \mathrm{t} / \mathrm{N}\right)-\operatorname{imag}(\operatorname{Ryh}(6)) * \sin (2 * \mathrm{pi} * 5 * \mathrm{t} / \mathrm{N})$;

$\mathrm{Rfh}=\mathrm{Rfh}+\operatorname{real}(\operatorname{Ryh}(9)) * \cos \left(2 * \mathrm{pi}^{*} 8 * \mathrm{t} / \mathrm{N}\right)-\operatorname{imag}(\operatorname{Ryh}(9)) * \sin (2 * \mathrm{pi} * 8 * \mathrm{t} / \mathrm{N})$;

$\mathrm{Rfh}=\mathrm{Rfh}+\operatorname{real}(\operatorname{Ryh}(11)) * \cos (2 * \mathrm{pi} * 10 * \mathrm{t} / \mathrm{N})-\operatorname{imag}(\operatorname{Ryh}(11)) * \sin (2 * \mathrm{pi} * 10 * \mathrm{t} / \mathrm{N})$;

$\operatorname{Rfk} 0=\operatorname{Ryk}(1) / 2$;

$\mathrm{Rfk}=\mathrm{Rfk} 0+\operatorname{real}(\operatorname{Ryk}(2)) * \cos (2 * \mathrm{pi} * \mathrm{t} / \mathrm{N})-\operatorname{imag}(\operatorname{Ryk}(2)) * \sin (2 * \mathrm{pi} * \mathrm{t} / \mathrm{N})$;

$\mathrm{Rfk}=\mathrm{Rfk}+\operatorname{real}(\operatorname{Ryk}(3)) * \cos (2 * \mathrm{pi} * 2 * \mathrm{t} / \mathrm{N})-\operatorname{imag}(\operatorname{Ryk}(3)) * \sin (2 * \mathrm{pi} * 2 * \mathrm{t} / \mathrm{N})$;

$\mathrm{Rfk}=\mathrm{Rfk}+\operatorname{real}(\operatorname{Ryk}(4)) * \cos \left(2 * \mathrm{pi}^{*} 3 * \mathrm{t} / \mathrm{N}\right)-\operatorname{imag}(\operatorname{Ryk}(4)) * \sin (2 * \mathrm{pi} * 3 * \mathrm{t} / \mathrm{N})$;

$\mathrm{Rfk}=\mathrm{Rfk}+\operatorname{real}(\operatorname{Ryk}(5)) * \cos (2 * \mathrm{pi} * 4 * \mathrm{t} / \mathrm{N})-\operatorname{imag}(\operatorname{Ryk}(5)) * \sin (2 * \mathrm{pi} * 4 * \mathrm{t} / \mathrm{N})$;

$\mathrm{Rfk}=\mathrm{Rfk}+\operatorname{real}(\operatorname{Ryk}(6)) * \cos \left(2 * \mathrm{pi}^{*} 5 * \mathrm{t} / \mathrm{N}\right)-\operatorname{imag}(\operatorname{Ryk}(6)) * \sin (2 * \mathrm{pi} * 5 * \mathrm{t} / \mathrm{N})$;

$\mathrm{Rfk}=\mathrm{Rfk}+\operatorname{real}(\operatorname{Ryk}(7)) * \cos \left(2 * \mathrm{pi}^{*} 6 * \mathrm{t} / \mathrm{N}\right)-\operatorname{imag}(\operatorname{Ryk}(7)) * \sin (2 * \mathrm{pi} * 6 * \mathrm{t} / \mathrm{N})$;

$\mathrm{Rfk}=\mathrm{Rfk}+\operatorname{real}(\operatorname{Ryk}(9)) * \cos (2 * \mathrm{pi} * 8 * \mathrm{t} / \mathrm{N})-\operatorname{imag}(\operatorname{Ryk}(9)) * \sin (2 * \mathrm{pi} * 8 * \mathrm{t} / \mathrm{N})$;

$\mathrm{Rfa} 0=\mathrm{Rya}(1) / 2$;

$\mathrm{Rfa}=\mathrm{Rfa} 0+\operatorname{real}(\mathrm{Rya}(2)) * \cos (2 * \mathrm{pi} * \mathrm{t} / \mathrm{N})-\operatorname{imag}(\operatorname{Rya}(2)) * \sin (2 * \mathrm{pi} * \mathrm{t} / \mathrm{N})$;

$\mathrm{Rfa}=\mathrm{Rfa}+\operatorname{real}(\operatorname{Rya}(3)) * \cos (2 * \mathrm{pi} * 2 * \mathrm{t} / \mathrm{N})-\operatorname{imag}(\operatorname{Rya}(3)) * \sin (2 * \mathrm{pi} * 2 * \mathrm{t} / \mathrm{N})$;

$\mathrm{Rfa}=\mathrm{Rfa}+\operatorname{real}(\operatorname{Rya}(4)) * \cos (2 * \mathrm{pi} * 3 * \mathrm{t} / \mathrm{N})-\operatorname{imag}(\operatorname{Rya}(4)) * \sin (2 * \mathrm{pi} * 3 * \mathrm{t} / \mathrm{N})$;

$\mathrm{Rfa}=\mathrm{Rfa}+\operatorname{real}(\operatorname{Rya}(5)) * \cos (2 * \mathrm{pi} * 4 * \mathrm{t} / \mathrm{N})-\operatorname{imag}(\operatorname{Rya}(5)) * \sin (2 * \mathrm{pi} * 4 * \mathrm{t} / \mathrm{N})$;

$\mathrm{Rfa}=\mathrm{Rfa}+\operatorname{real}(\operatorname{Rya}(6)) * \cos (2 * \mathrm{pi} * 5 * \mathrm{t} / \mathrm{N})-\mathrm{imag}(\operatorname{Rya}(6)) * \sin (2 * \mathrm{pi} * 5 * \mathrm{t} / \mathrm{N})$;

$\mathrm{Rfa}=\mathrm{Rfa}+\operatorname{real}(\operatorname{Rya}(8)) * \cos (2 * \mathrm{pi} * 7 * \mathrm{t} / \mathrm{N})-\operatorname{imag}(\operatorname{Rya}(8)) * \sin (2 * \mathrm{pi} * 7 * \mathrm{t} / \mathrm{N})$; 







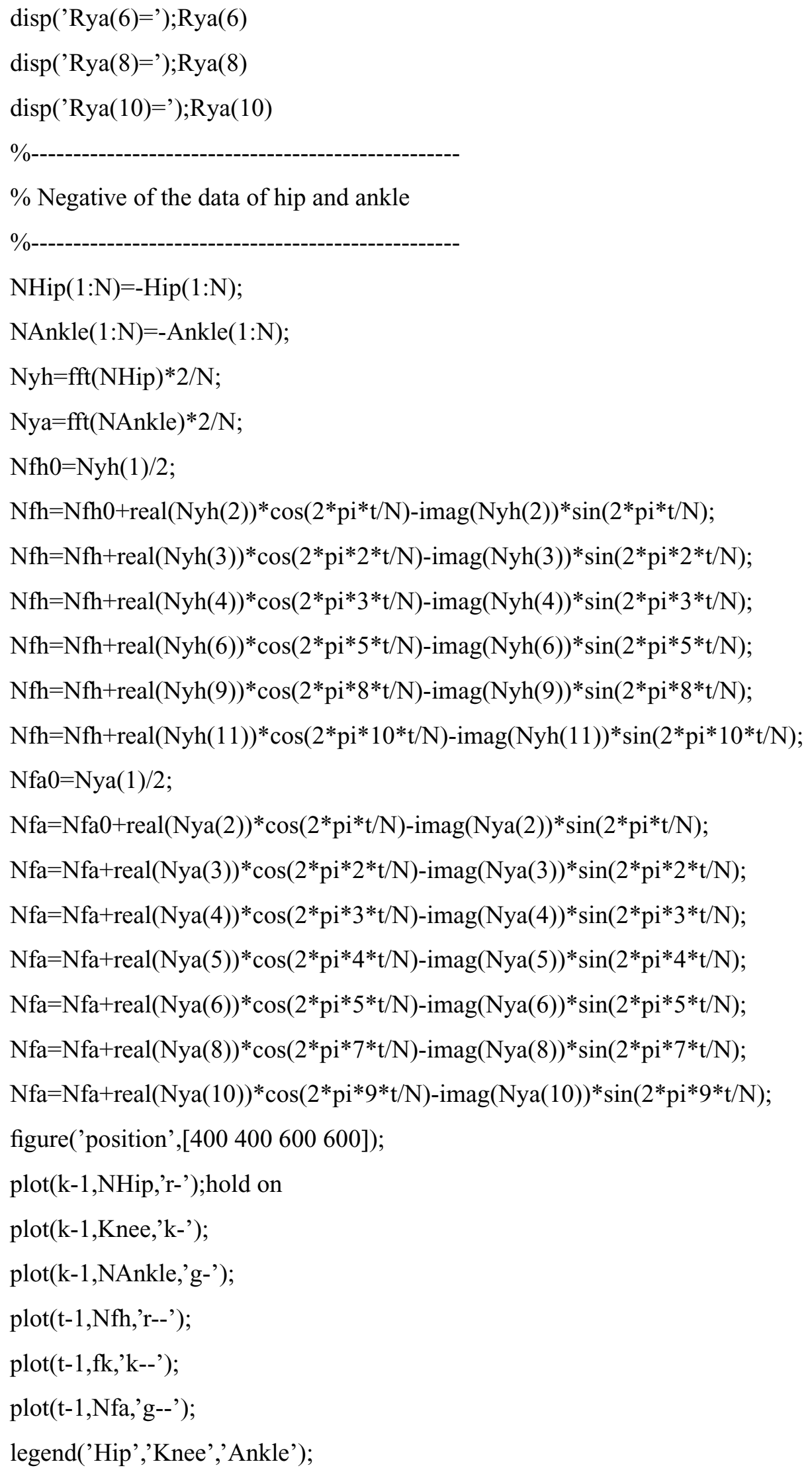







$\mathrm{RNfh}=\mathrm{RNfh}+\operatorname{real}(\mathrm{RNyh}(4)) * \cos \left(2 * \mathrm{pi}^{*} 3 * \mathrm{t} / \mathrm{N}\right)-\operatorname{imag}(\mathrm{RNyh}(4)) * \sin (2 * \mathrm{pi} * 3 * \mathrm{t} / \mathrm{N})$;

$\mathrm{RNfh}=\mathrm{RNfh}+\operatorname{real}(\mathrm{RNyh}(6)) * \cos (2 * \mathrm{pi} * 5 * \mathrm{t} / \mathrm{N})-\operatorname{imag}(\mathrm{RNyh}(6)) * \sin (2 * \mathrm{pi} * 5 * \mathrm{t} / \mathrm{N})$;

$\mathrm{RNfh}=\mathrm{RNfh}+\operatorname{real}(\mathrm{RNyh}(9)) * \cos (2 * \mathrm{pi} * 8 * \mathrm{t} / \mathrm{N})-\operatorname{imag}(\mathrm{RNyh}(9)) * \sin (2 * \mathrm{pi} * 8 * \mathrm{t} / \mathrm{N})$;

$\mathrm{RNfh}=\mathrm{RNfh}+\operatorname{real}(\mathrm{RNyh}(11)) * \cos (2 * \mathrm{pi} * 10 * \mathrm{t} / \mathrm{N})-\operatorname{imag}(\mathrm{RNyh}(11)) * \sin (2 * \mathrm{pi} * 10 * \mathrm{t} / \mathrm{N})$;

$\mathrm{RNfa} 0=\mathrm{RNya}(1) / 2$;

$\mathrm{RNfa}=\mathrm{RNfa} 0+\operatorname{real}(\mathrm{RNya}(2)) * \cos (2 * \mathrm{pi} * \mathrm{t} / \mathrm{N})-\operatorname{imag}(\mathrm{RNya}(2)) * \sin (2 * \mathrm{pi} * \mathrm{t} / \mathrm{N})$;

$\mathrm{RNfa}=\mathrm{RNfa}+\mathrm{real}(\mathrm{RNya}(3)) * \cos (2 * \mathrm{pi} * 2 * \mathrm{t} / \mathrm{N})-\operatorname{imag}(\mathrm{RNya}(3)) * \sin (2 * \mathrm{pi} * 2 * \mathrm{t} / \mathrm{N})$;

$\mathrm{RNfa}=\mathrm{RNfa}+\mathrm{real}(\mathrm{RNya}(4)) * \cos (2 * \mathrm{pi} * 3 * \mathrm{t} / \mathrm{N})-\operatorname{imag}(\mathrm{RNya}(4)) * \sin \left(2 * \mathrm{pi}^{*} 3 * \mathrm{t} / \mathrm{N}\right)$;

$\mathrm{RNfa}=\mathrm{RNfa}+\mathrm{real}(\mathrm{RNya}(5)) * \cos (2 * \mathrm{pi} * 4 * \mathrm{t} / \mathrm{N})-\operatorname{imag}(\mathrm{RNya}(5)) * \sin (2 * \mathrm{pi} * 4 * \mathrm{t} / \mathrm{N})$;

$\mathrm{RNfa}=\mathrm{RNfa}+\mathrm{real}(\mathrm{RNya}(6)) * \cos (2 * \mathrm{pi} * 5 * \mathrm{t} / \mathrm{N})-\operatorname{imag}(\mathrm{RNya}(6)) * \sin (2 * \mathrm{pi} * 5 * \mathrm{t} / \mathrm{N})$;

$\mathrm{RNfa}=\mathrm{RNfa}+\mathrm{real}(\mathrm{RNya}(8)) * \cos (2 * \mathrm{pi} * 7 * \mathrm{t} / \mathrm{N})-\operatorname{imag}(\mathrm{RNya}(8)) * \sin (2 * \mathrm{pi} * 7 * \mathrm{t} / \mathrm{N})$;

$\mathrm{RNfa}=\mathrm{RNfa}+\mathrm{real}(\mathrm{RNya}(10)) * \cos (2 * \mathrm{pi} * 9 * \mathrm{t} / \mathrm{N})-\operatorname{imag}(\mathrm{RNya}(10)) * \sin (2 * \mathrm{pi} * 9 * \mathrm{t} / \mathrm{N})$;

figure('position',[450 450600600$]$ );

plot(k-1,RNHip,'r-'); hold on

plot(k-1,RKnee,'k-');

plot(k-1,RNAnkle,'g-');

plot(t-1,RNfh,'r--');

plot(t-1,Rfk,'k--');

plot(t-1,RNfa,'g--');

legend('Hip','Knee','Ankle');

disp('Angle data for right foot after the Negative of data of hip and ankle');

RNfh0

$\operatorname{disp}\left({ }^{\prime} \mathrm{RNyh}(2)={ }^{\prime}\right) ; \mathrm{RNyh}(2)$

$\operatorname{disp}(' R N y h(3)=') ; R N y h(3)$

$\operatorname{disp}(' R N y h(4)=') ; R N y h(4)$

$\operatorname{disp}(' R N y h(6)=') ; R N y h(6)$

$\operatorname{disp}(' R N y h(9)=') ; R N y h(9)$

$\operatorname{disp}(' R N y h(11)=') ; R N y h(11)$

Rfk0

$\operatorname{disp}\left({ }^{\prime} \operatorname{RNyk}(2)='\right) ; \operatorname{Ryk}(2)$

$\operatorname{disp}\left(\operatorname{RNyk}^{\prime}(3)='\right) ; \operatorname{Ryk}(3)$

$\operatorname{disp}(' \operatorname{RNyk}(4)=') ; \operatorname{Ryk}(4)$

$\operatorname{disp}\left({ }^{\prime} \operatorname{RNyk}(5)='\right) ; \operatorname{Ryk}(5)$ 





subplot(3,2,2); plot((k-1)*2,RNHip,'r-'`); hold on

$\operatorname{plot}\left((\mathrm{t}-1) * 2, \mathrm{RNfh},{ }^{\prime} \mathrm{k}-\mathrm{-}^{\prime}\right)$;

xlabel('Stride \%');

ylabel('Degree');

grid on;

title('Right Hip angle')

legend ('Measured','Fourier');

subplot $(3,2,4) ; \operatorname{plot}\left((\mathrm{k}-1)^{*} 2, \mathrm{RKnee}^{\prime}, \mathrm{r}-{ }^{\prime}\right)$; hold on

$\operatorname{plot}\left((\mathrm{t}-1) * 2, \mathrm{Rfk},{ }^{\prime} \mathrm{k}-\mathrm{-}^{\prime}\right)$;

xlabel('Stride \%');

ylabel('Degree');

grid on;

title('Right Knee angle');

legend ('Measured','Fourier');

subplot(3,2,6); plot((k-1)*2,RNAnkle,'r-'); hold on

$\operatorname{plot}\left((\mathrm{t}-1) * 2, \mathrm{RNfa},{ }^{\prime} \mathrm{b}-\mathrm{-}\right)$;

xlabel('Stride \%');

ylabel('Degree');

legend ('Measured','Fourier');

grid on;

title('Right Ankle angle');

\section{B.2 bezier.m}

This code is compiled to fit points of some key positions to bezier curve. We have no data for movement of arm and shoulder, but just estimate some key positions, then fit it to bezier curve.

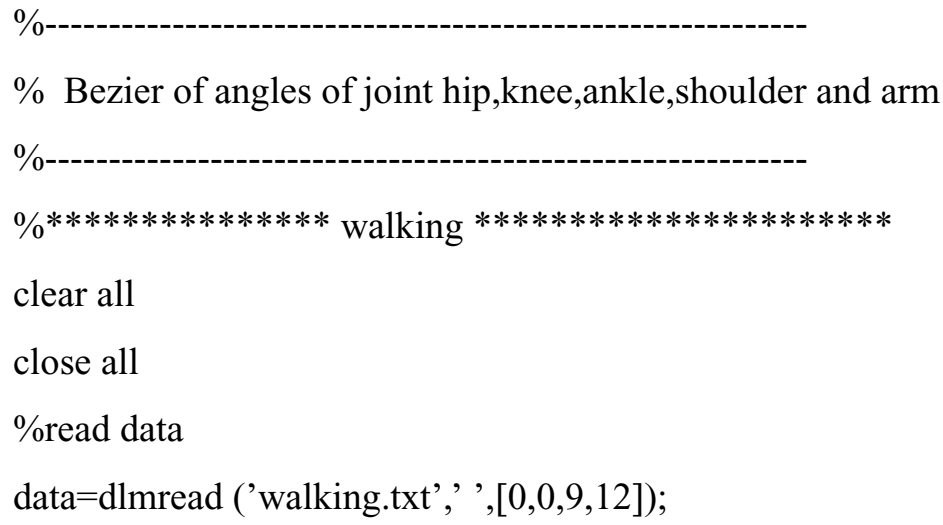




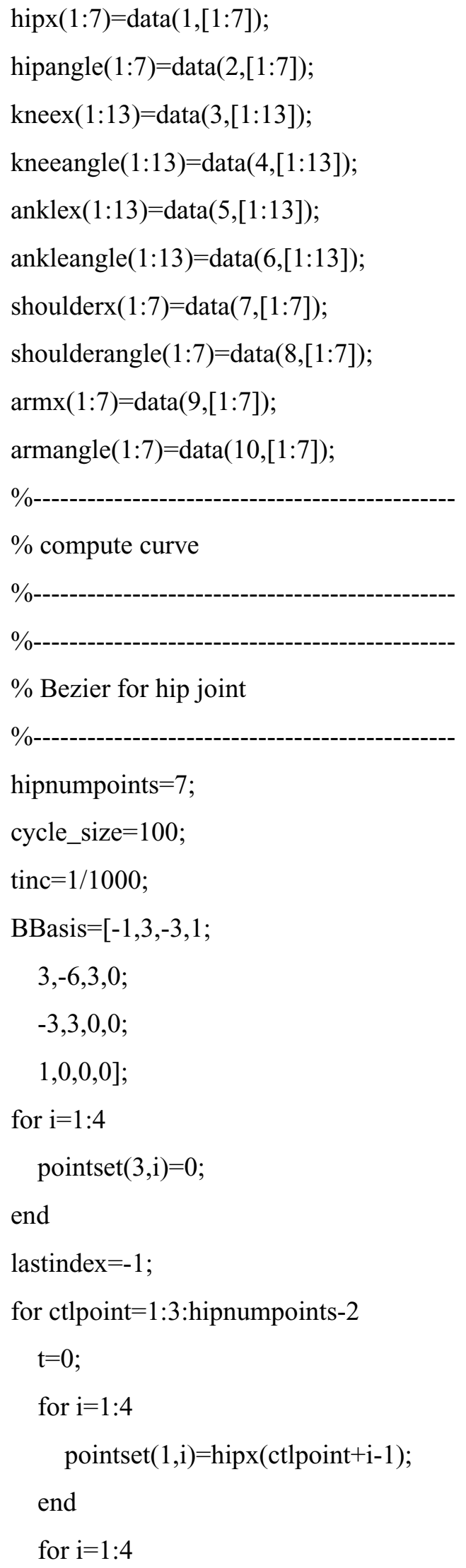




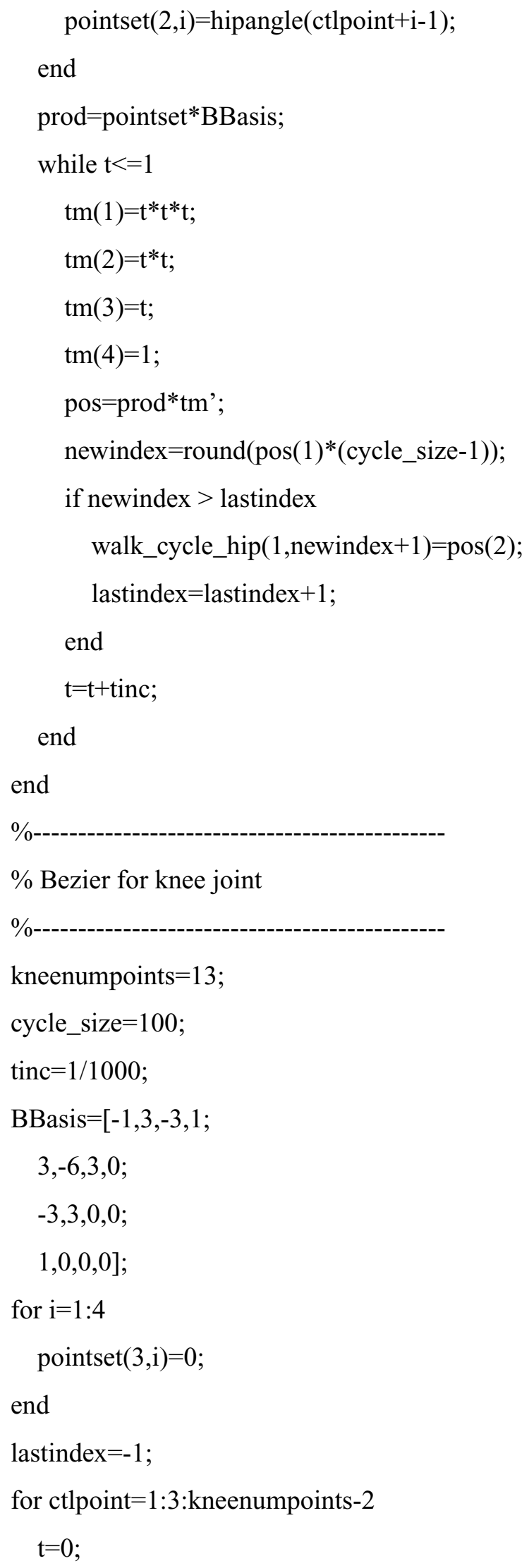




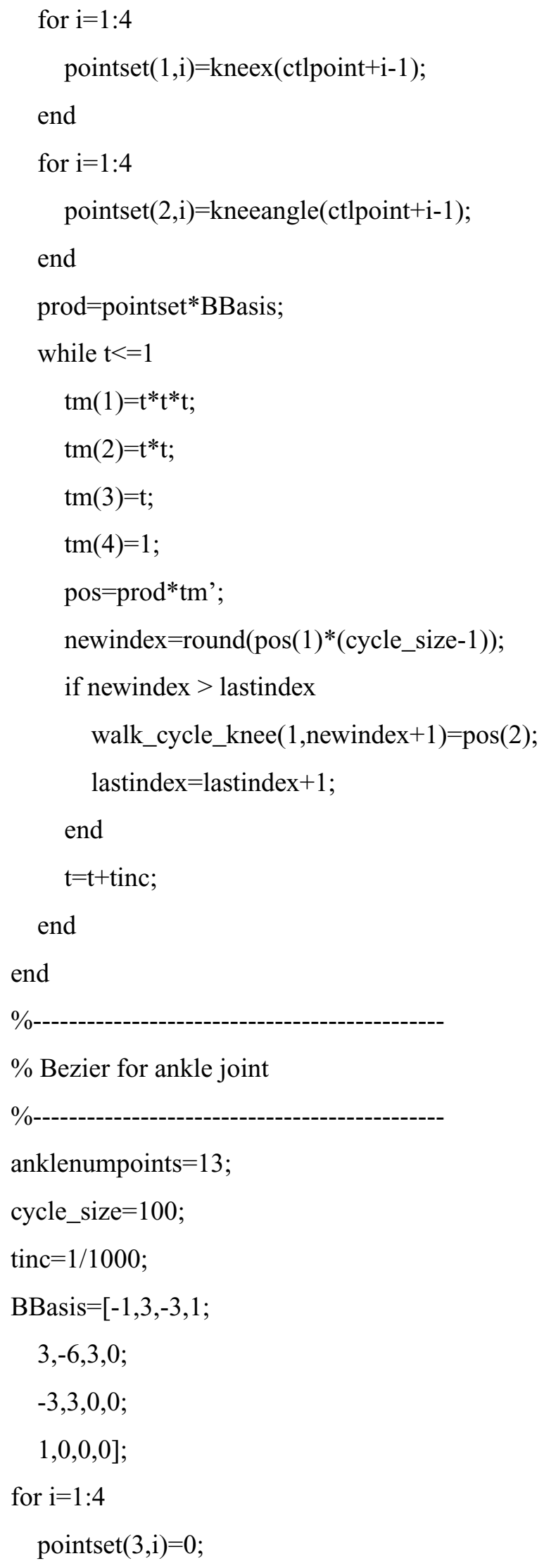









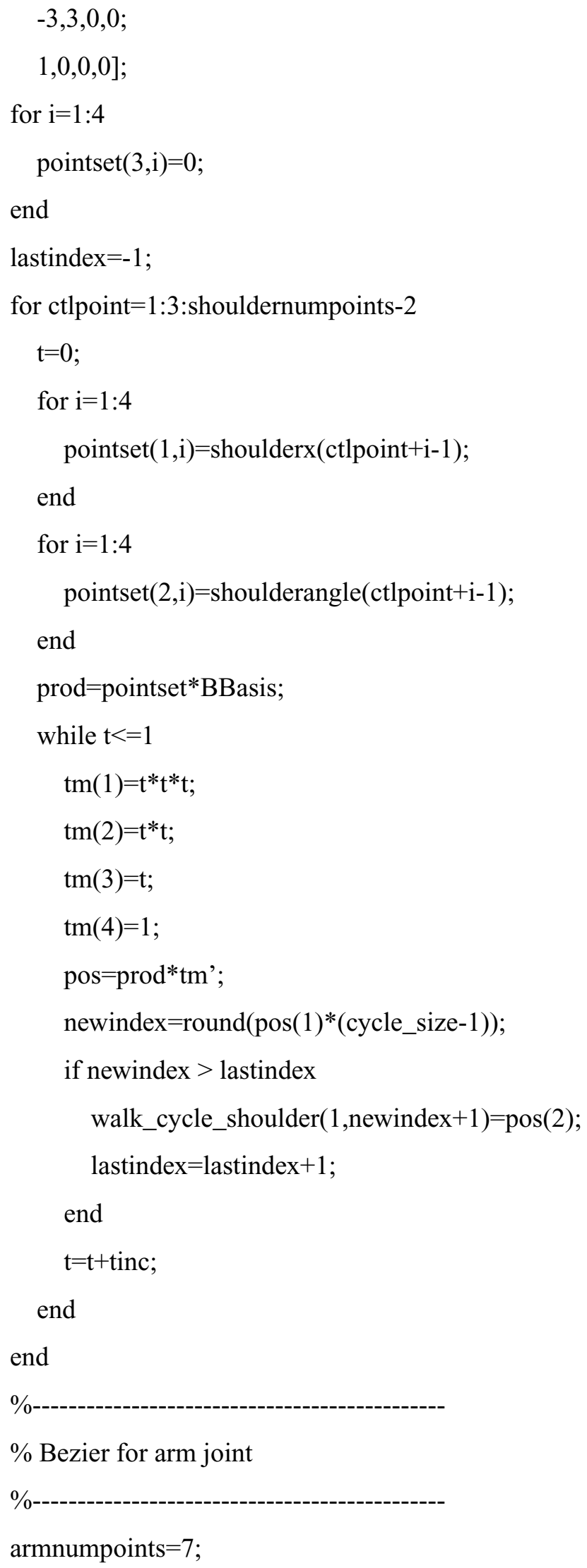




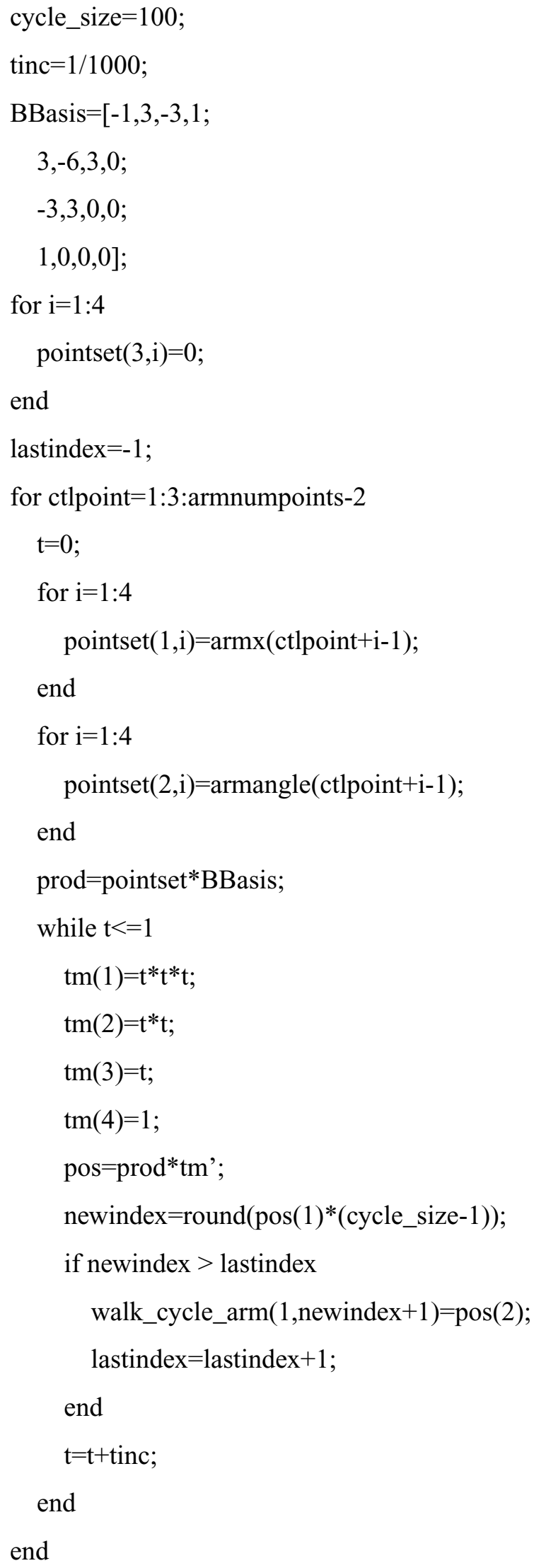


figure('position',[0 0600 600]);

plot(hipx,hipangle,'r-'); hold on

$\operatorname{plot}([1: 100] / 100$,walk_cycle_hip,'r--’');

plot(kneex,kneeangle,'k-');

$\operatorname{plot}([1: 100] / 100$,walk_cycle_knee,'k--'),

plot(anklex,ankleangle,'g-');

plot([1:100]/100,walk_cycle_ankle,'g--'),

plot(shoulderx,shoulderangle,'b-');

plot([1:100]/100,walk_cycle_shoulder,'b--'),

plot(armx,armangle,'c-');

$\operatorname{plot}([1: 100] / 100$,walk_cycle_arm,'c--'),

legend('Hip',,',Knee',','Ankle',,',Shoulder',,',Arm');

$\%$

$\% * * * * * * * * * * * * * * *$ running $* * * * * * * * * * * * * * * * * * * * * *$

\%-------------------------------------------------

$\%$ read data

data=dlmread ('running.txt', ', , $[0,0,9,12])$;

$\operatorname{hipx}(1: 7)=\operatorname{data}(1,[1: 7])$;

hipangle $(1: 7)=\operatorname{data}(2,[1: 7])$;

$\operatorname{kneex}(1: 7)=\operatorname{data}(3,[1: 7])$;

kneeangle $(1: 7)=\operatorname{data}(4,[1: 7])$;

$\operatorname{anklex}(1: 13)=\operatorname{data}(5,[1: 13])$;

ankleangle $(1: 13)=\operatorname{data}(6,[1: 13])$;

shoulderx $(1: 7)=\operatorname{data}(7,[1: 7])$;

shoulderangle $(1: 7)=\operatorname{data}(8,[1: 7])$;

$\operatorname{armx}(1: 7)=\operatorname{data}(9,[1: 7])$;

$\operatorname{armangle}(1: 7)=\operatorname{data}(10,[1: 7])$;

\%------------------------------------------------

$\%$ compute curve

\%------------------------------------------------

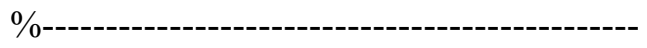

$\%$ Bezier for hip joint

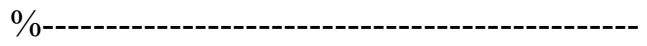









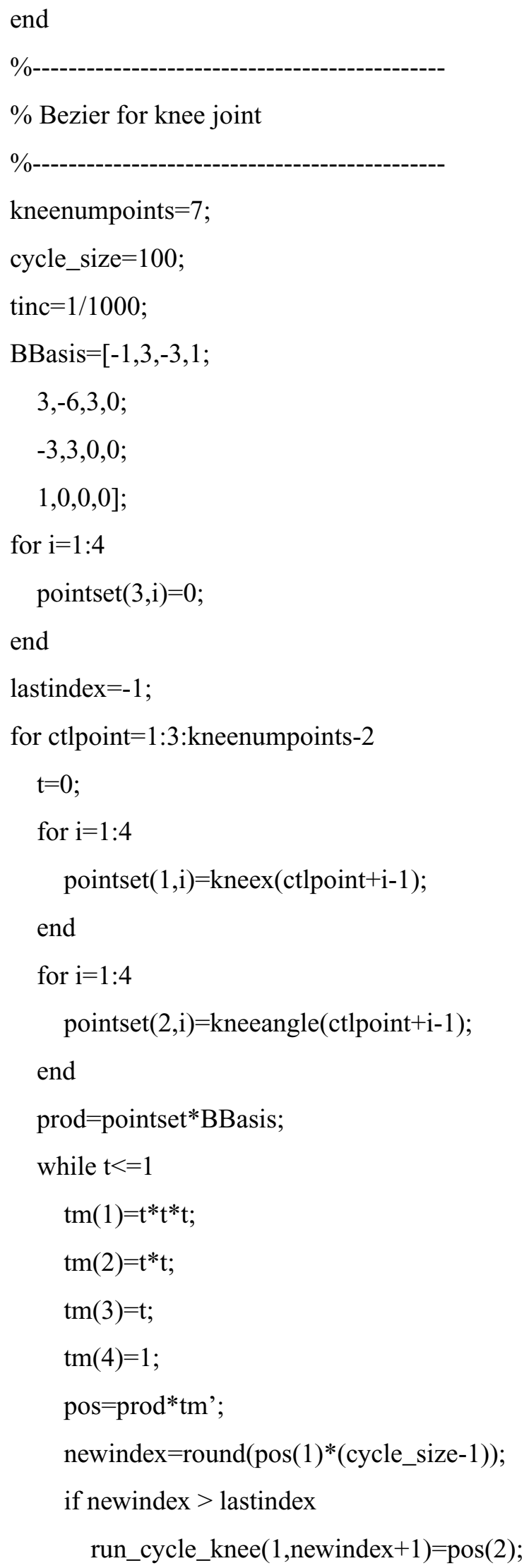




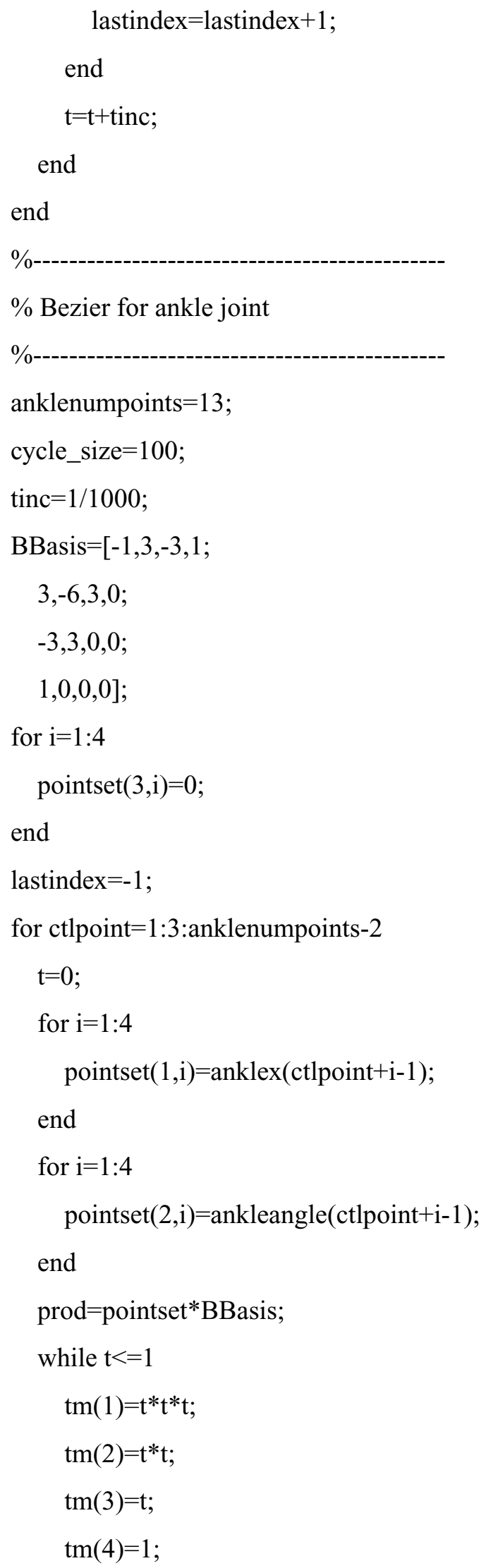




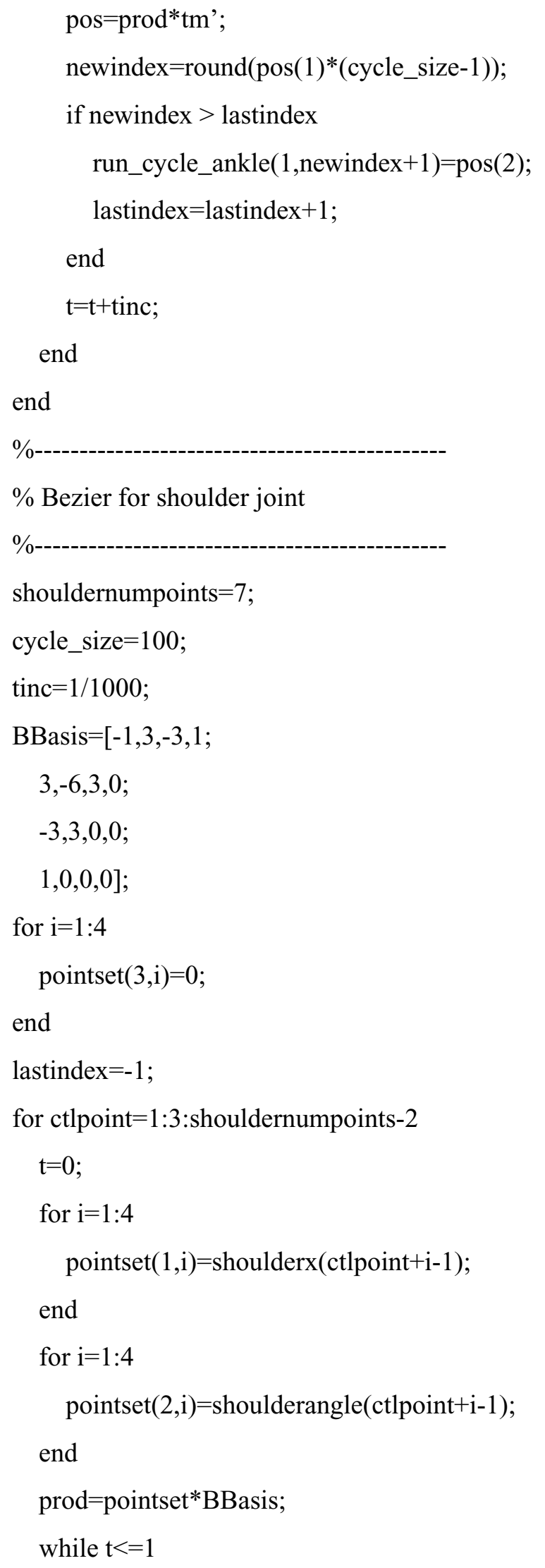




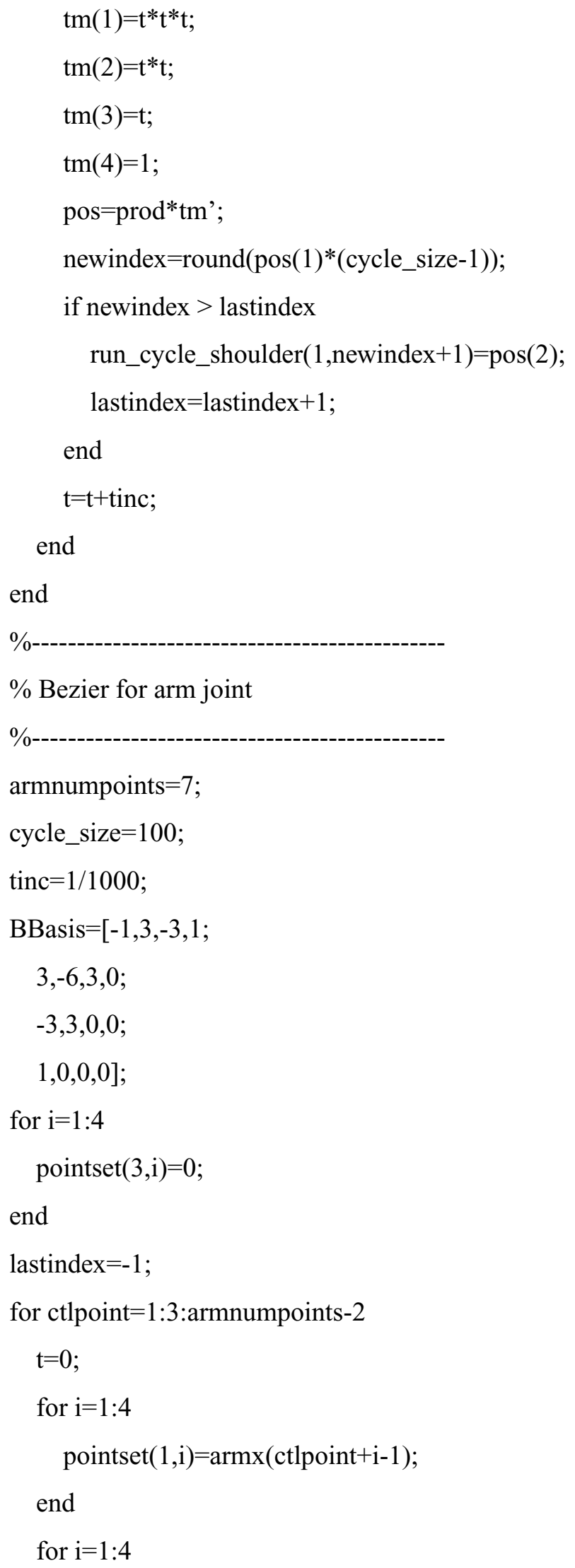




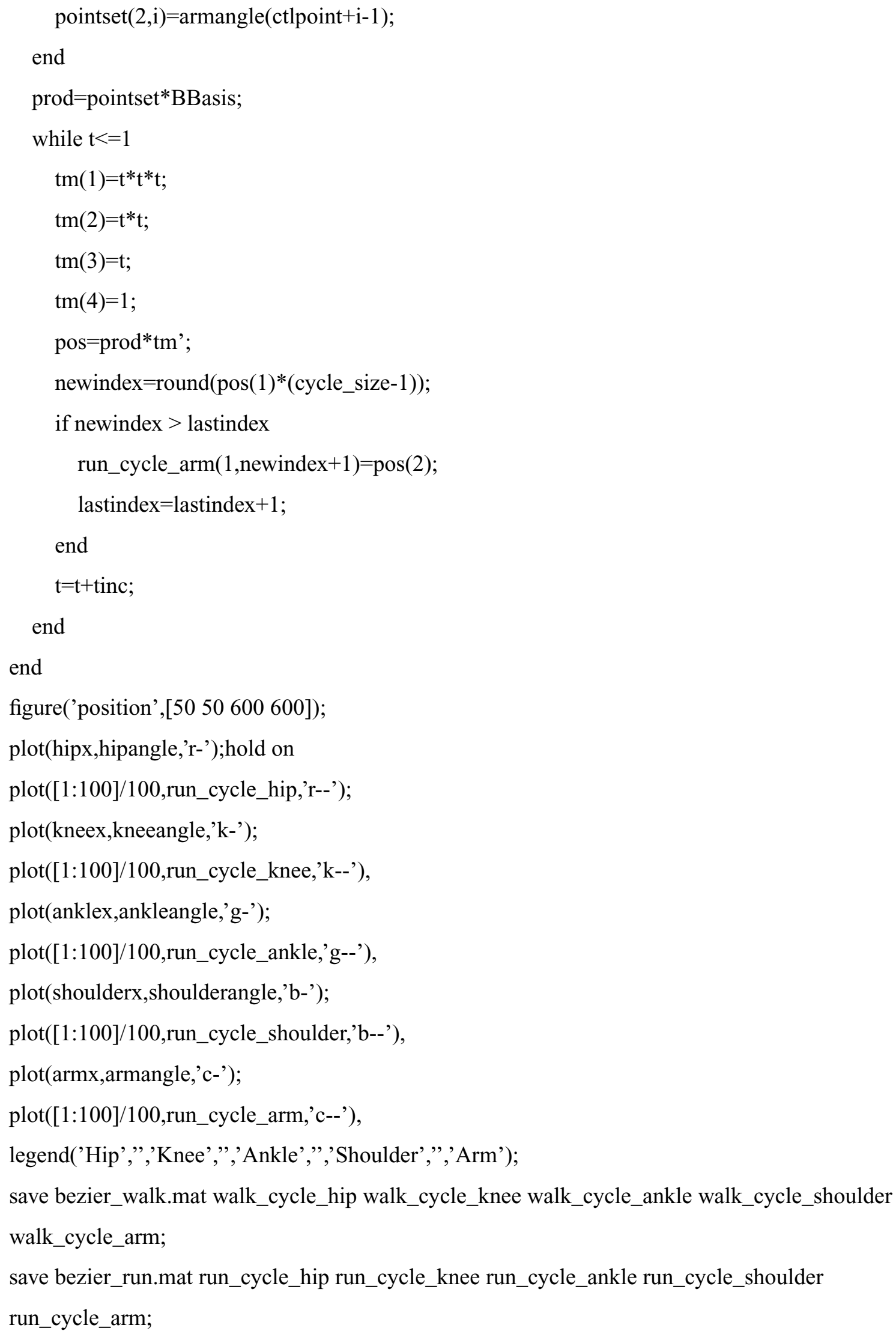




\section{B.3 bezier_regression.m}

This code is compiled to fit the bezier curve with Fourier series, then it can be a peoredical function.

$\%$ Regression of angles of hip,knee, ankle,shoulder and arm

clear all

close all

\%load data from bezeir calculation

load bezier_walk.mat;

Hip=walk_cycle_hip;

Knee=walk_cycle_knee;

Ankle=walk_cycle_ankle;

Shoulder=walk_cycle_shoulder;

Elbow=walk_cycle_arm;

$\mathrm{N}=$ length(Hip);

$\mathrm{k}=1: \mathrm{N}$;

$\mathrm{yh}=\mathrm{fft}(\mathrm{Hip}) * 2 / \mathrm{N}$;

$\mathrm{yk}=\mathrm{fft}($ Knee $) * 2 / \mathrm{N}$;

$\mathrm{ya}=\mathrm{fft}($ Ankle) $* 2 / \mathrm{N}$;

$\mathrm{ys}=\mathrm{fft}($ Shoulder) $* 2 / \mathrm{N}$;

$\mathrm{ye}=\mathrm{fft}($ Elbow $) * 2 / \mathrm{N}$;

$\mathrm{t}=1: \mathrm{N}$;

fh $0=0$;

for $\mathrm{i}=1: \mathrm{N}-1$

fh0 $=$ fh0+Hip(i);

end

fh $0=y h(1) / 2$;

$\mathrm{fh}=\mathrm{fh} 0+\mathrm{real}(\mathrm{yh}(2)) * \cos (2 * \mathrm{pi} * \mathrm{t} / \mathrm{N})-\mathrm{imag}(\mathrm{yh}(2)) * \sin (2 * \mathrm{pi} * \mathrm{t} / \mathrm{N})$;

$\mathrm{fh}=\mathrm{fh}+\mathrm{real}(\mathrm{yh}(3)) * \cos (2 * \mathrm{pi} * 2 * \mathrm{t} / \mathrm{N})-\operatorname{imag}(\mathrm{yh}(3)) * \sin (2 * \mathrm{pi} * 2 * \mathrm{t} / \mathrm{N})$;

$\mathrm{fh}=\mathrm{fh}+\mathrm{real}(\mathrm{yh}(4)) * \cos (2 * \mathrm{pi} * 3 * \mathrm{t} / \mathrm{N})-\operatorname{imag}(\mathrm{yh}(4)) * \sin (2 * \mathrm{pi} * 3 * \mathrm{t} / \mathrm{N})$;

$\mathrm{fh}=\mathrm{fh}+\mathrm{real}(\mathrm{yh}(5)) * \cos (2 * \mathrm{pi} * 4 * \mathrm{t} / \mathrm{N})-\operatorname{imag}(\mathrm{yh}(5)) * \sin \left(2 * \mathrm{pi}^{*} 4 * \mathrm{t} / \mathrm{N}\right)$;

$\mathrm{fh}=\mathrm{fh}+\mathrm{real}(\mathrm{yh}(6)) * \cos (2 * \mathrm{pi} * 5 * \mathrm{t} / \mathrm{N})-\operatorname{imag}(\mathrm{yh}(6)) * \sin (2 * \mathrm{pi} * 5 * \mathrm{t} / \mathrm{N})$;

$\mathrm{fh}=\mathrm{fh}+\mathrm{real}(\mathrm{yh}(7)) * \cos (2 * \mathrm{pi} * 6 * \mathrm{t} / \mathrm{N})-\operatorname{imag}(\mathrm{yh}(7)) * \sin (2 * \mathrm{pi} * 6 * \mathrm{t} / \mathrm{N})$; 
$\mathrm{fk} 0=\mathrm{yk}(1) / 2$;

$\mathrm{fk}=\mathrm{fk} 0+\mathrm{real}(\mathrm{yk}(2)) * \cos \left(2 * \mathrm{pi}^{*} \mathrm{t} / \mathrm{N}\right)-\operatorname{imag}(\mathrm{yk}(2)) * \sin (2 * \mathrm{pi} * \mathrm{t} / \mathrm{N})$;

$\mathrm{fk}=\mathrm{fk}+\mathrm{real}(\mathrm{yk}(3)) * \cos (2 * \mathrm{pi} * 2 * \mathrm{t} / \mathrm{N})-\operatorname{imag}(\mathrm{yk}(3)) * \sin (2 * \mathrm{pi} * 2 * \mathrm{t} / \mathrm{N})$;

$\mathrm{fk}=\mathrm{fk}+\mathrm{real}(\mathrm{yk}(4)) * \cos (2 * \mathrm{pi} * 3 * \mathrm{t} / \mathrm{N})-\operatorname{imag}(\mathrm{yk}(4)) * \sin (2 * \mathrm{pi} * 3 * \mathrm{t} / \mathrm{N})$;

$\mathrm{fk}=\mathrm{fk}+\mathrm{real}(\mathrm{yk}(5)) * \cos (2 * \mathrm{pi} * 4 * \mathrm{t} / \mathrm{N})-\operatorname{imag}(\mathrm{yk}(5)) * \sin \left(2 * \mathrm{pi}^{*} 4 * \mathrm{t} / \mathrm{N}\right)$;

$\mathrm{fk}=\mathrm{fk}+\mathrm{real}(\mathrm{yk}(6)) * \cos (2 * \mathrm{pi} * 5 * \mathrm{t} / \mathrm{N})-\operatorname{imag}(\mathrm{yk}(6)) * \sin (2 * \mathrm{pi} * 5 * \mathrm{t} / \mathrm{N})$;

$\mathrm{fk}=\mathrm{fk}+\mathrm{real}(\mathrm{yk}(7)) * \cos (2 * \mathrm{pi} * 6 * \mathrm{t} / \mathrm{N})-\operatorname{imag}(\mathrm{yk}(7)) * \sin \left(2 * \mathrm{pi}^{*} 6 * \mathrm{t} / \mathrm{N}\right)$;

$\mathrm{fk}=\mathrm{fk}+\mathrm{real}(\mathrm{yk}(8)) * \cos (2 * \mathrm{pi} * 7 * \mathrm{t} / \mathrm{N})-\operatorname{imag}(\mathrm{yk}(8)) * \sin \left(2 * \mathrm{pi}^{*} 7 * \mathrm{t} / \mathrm{N}\right)$;

$\mathrm{fa} 0=\mathrm{ya}(1) / 2$;

$\mathrm{fa}=\mathrm{fa} 0+\mathrm{real}(\mathrm{ya}(2)) * \cos (2 * \mathrm{pi} * \mathrm{t} / \mathrm{N})-\operatorname{imag}(\mathrm{ya}(2)) * \sin (2 * \mathrm{pi} * \mathrm{t} / \mathrm{N})$;

$\mathrm{fa}=\mathrm{fa}+\operatorname{real}(\mathrm{ya}(3)) * \cos (2 * \mathrm{pi} * 2 * \mathrm{t} / \mathrm{N})-\operatorname{imag}(\mathrm{ya}(3)) * \sin (2 * \mathrm{pi} * 2 * \mathrm{t} / \mathrm{N})$;

$\mathrm{fa}=\mathrm{fa}+\operatorname{real}(\mathrm{ya}(4)) * \cos (2 * \mathrm{pi} * 3 * \mathrm{t} / \mathrm{N})-\operatorname{imag}(\mathrm{ya}(4)) * \sin (2 * \mathrm{pi} * 3 * \mathrm{t} / \mathrm{N})$;

$\mathrm{fa}=\mathrm{fa}+\operatorname{real}(\mathrm{ya}(5)) * \cos (2 * \mathrm{pi} * 4 * \mathrm{t} / \mathrm{N})-\operatorname{imag}(\mathrm{ya}(5)) * \sin (2 * \mathrm{pi} * 4 * \mathrm{t} / \mathrm{N})$;

$\mathrm{fa}=\mathrm{fa}+\operatorname{real}(\mathrm{ya}(6)) * \cos (2 * \mathrm{pi} * 5 * \mathrm{t} / \mathrm{N})-\operatorname{imag}(\mathrm{ya}(6)) * \sin (2 * \mathrm{pi} * 5 * \mathrm{t} / \mathrm{N})$;

$\mathrm{fa}=\mathrm{fa}+\operatorname{real}(\mathrm{ya}(7)) * \cos (2 * \mathrm{pi} * 6 * \mathrm{t} / \mathrm{N})-\operatorname{imag}(\mathrm{ya}(7)) * \sin (2 * \mathrm{pi} * 6 * \mathrm{t} / \mathrm{N})$;

$\mathrm{fa}=\mathrm{fa}+\operatorname{real}(\mathrm{ya}(8)) * \cos \left(2 * \mathrm{pi}^{*} 7 * \mathrm{t} / \mathrm{N}\right)-\operatorname{imag}(\mathrm{ya}(8)) * \sin (2 * \mathrm{pi} * 7 * \mathrm{t} / \mathrm{N})$;

$\mathrm{fa}=\mathrm{fa}+\mathrm{real}(\mathrm{ya}(9)) * \cos \left(2 * \mathrm{pi}^{*} 8 * \mathrm{t} / \mathrm{N}\right)-\operatorname{imag}(\mathrm{ya}(9)) * \sin (2 * \mathrm{pi} * 8 * \mathrm{t} / \mathrm{N})$;

$\mathrm{fa}=\mathrm{fa}+\mathrm{real}(\mathrm{ya}(10)) * \cos \left(2 * \mathrm{pi}^{*} 9 * \mathrm{t} / \mathrm{N}\right)-\operatorname{imag}(\mathrm{ya}(10)) * \sin (2 * \mathrm{pi} * \mathrm{~g} * \mathrm{t} / \mathrm{N})$;

$\mathrm{fs} 0=\mathrm{ys}(1) / 2$;

$\mathrm{fs}=\mathrm{fs} 0+\operatorname{real}(\mathrm{ys}(2)) * \cos (2 * \mathrm{pi} * \mathrm{t} / \mathrm{N})-\operatorname{imag}(\mathrm{ys}(2)) * \sin (2 * \mathrm{pi} * \mathrm{t} / \mathrm{N})$;

$\mathrm{fs}=\mathrm{fs}+\operatorname{real}(\mathrm{ys}(3)) * \cos \left(2 * \mathrm{pi}^{*} 2 * \mathrm{t} / \mathrm{N}\right)-\operatorname{imag}(\mathrm{ys}(3)) * \sin \left(2 * \mathrm{pi}^{*} 2 * \mathrm{t} / \mathrm{N}\right)$;

$\mathrm{fs}=\mathrm{fs}+\operatorname{real}(\mathrm{ys}(4)) * \cos (2 * \mathrm{pi} * 3 * \mathrm{t} / \mathrm{N})-\operatorname{imag}(\mathrm{ys}(4)) * \sin \left(2 * \mathrm{pi}^{*} 3 * \mathrm{t} / \mathrm{N}\right)$;

$\mathrm{fs}=\mathrm{fs}+\mathrm{real}(\mathrm{ys}(5)) * \cos (2 * \mathrm{pi} * 4 * \mathrm{t} / \mathrm{N})-\operatorname{imag}(\mathrm{ys}(5)) * \sin (2 * \mathrm{pi} * 4 * \mathrm{t} / \mathrm{N})$;

$\mathrm{fs}=\mathrm{fs}+\operatorname{real}(\mathrm{ys}(6)) * \cos \left(2 * \mathrm{pi}^{*} 5 * \mathrm{t} / \mathrm{N}\right)-\operatorname{imag}(\mathrm{ys}(6)) * \sin \left(2 * \mathrm{pi}^{*} 5 * \mathrm{t} / \mathrm{N}\right)$;

fe $0=y e(1) / 2$;

$\mathrm{fe}=\mathrm{fe} 0+\mathrm{real}(\mathrm{ye}(2)) * \cos (2 * \mathrm{pi} * \mathrm{t} / \mathrm{N})-\operatorname{imag}(\mathrm{ye}(2)) * \sin (2 * \mathrm{pi} * \mathrm{t} / \mathrm{N})$;

$\mathrm{fe}=\mathrm{fe}+\operatorname{real}(\mathrm{ye}(3)) * \cos \left(2 * \mathrm{pi}^{*} 2 * \mathrm{t} / \mathrm{N}\right)-\operatorname{imag}(\mathrm{ye}(3)) * \sin (2 * \mathrm{pi} * 2 * \mathrm{t} / \mathrm{N})$;

$\mathrm{fe}=\mathrm{fe}+\mathrm{real}(\mathrm{ye}(4)) * \cos (2 * \mathrm{pi} * 3 * \mathrm{t} / \mathrm{N})-\operatorname{imag}(\mathrm{ye}(4)) * \sin (2 * \mathrm{pi} * 3 * \mathrm{t} / \mathrm{N})$;

$\mathrm{fe}=\mathrm{fe}+\operatorname{real}(\mathrm{ye}(5)) * \cos (2 * \mathrm{pi} * 4 * \mathrm{t} / \mathrm{N})-\operatorname{imag}(\mathrm{ye}(5)) * \sin (2 * \mathrm{pi} * 4 * \mathrm{t} / \mathrm{N})$;

$\mathrm{fe}=\mathrm{fe}+\operatorname{real}(\mathrm{ye}(6)) * \cos \left(2 * \mathrm{pi}^{*} 5 * \mathrm{t} / \mathrm{N}\right)-\operatorname{imag}(\mathrm{ye}(6)) * \sin (2 * \mathrm{pi} * 5 * \mathrm{t} / \mathrm{N})$;

$\mathrm{Fh}=\mathrm{yh}(1) / 2$;

$\mathrm{Fk}=\mathrm{yk}(1) / 2$;

$\mathrm{Fa}=\mathrm{ya}(1) / 2$; 
for $\mathrm{i}=1: \mathrm{N} / 2$

$\mathrm{Fh}=\mathrm{Fh}+\operatorname{real}(\mathrm{yh}(\mathrm{i}+1)) * \cos \left(2 * \mathrm{pi}^{*}{ }^{*} * \mathrm{t} / \mathrm{N}\right)-\operatorname{imag}(\mathrm{yh}(\mathrm{i}+1)) * \sin (2 * \mathrm{pi} * \mathrm{i} * \mathrm{t} / \mathrm{N}) ;$

$\mathrm{Fk}=\mathrm{Fk}+\operatorname{real}(\mathrm{yk}(\mathrm{i}+1)) * \cos \left(2 * \mathrm{pi}^{*}{ }^{\mathrm{i}} * \mathrm{t} / \mathrm{N}\right)-\operatorname{imag}(\mathrm{yk}(\mathrm{i}+1)) * \sin \left(2 * \mathrm{pi}^{*}{ }_{\mathrm{i}} * \mathrm{t} / \mathrm{N}\right)$;

$\mathrm{Fa}=\mathrm{Fa}+\mathrm{real}(\mathrm{ya}(\mathrm{i}+1)) * \cos \left(2 * \mathrm{pi}^{*} \mathrm{i} * \mathrm{t} / \mathrm{N}\right)-\mathrm{imag}(\mathrm{ya}(\mathrm{i}+1)) * \sin \left(2 * \mathrm{pi}^{*} \mathrm{i} * \mathrm{t} / \mathrm{N}\right) ;$

end

figure('position',[0 0600 600]);

plot(k-1,Hip,'r-'); hold on

plot(k-1,Knee,'k-');

plot(k-1,Ankle,'g-');

plot(k-1,Shoulder,'b-');

plot(k-1,Elbow,'c-');

plot(t-1,fh,'r--');

$\operatorname{plot}\left(\mathrm{t}-1, \mathrm{fk},{ }^{\prime} \mathrm{k}--^{\prime}\right)$;

plot(t-1,fa,'g--');

$\operatorname{plot}\left(\mathrm{t}-1, \mathrm{fs}, \mathrm{b}^{* \prime}\right)$;

plot(t-1,fe,'c*');

plot(t-1,Fh,'b--');

plot(t-1,Fk,'c--');

plot(t-1,Fa,'y--');

legend('Hip','Knee','Ankle','Shoulder','Elbow');

title('Leftside of walking_cycle');

grid on;

figure('position',[50 50600 600]);

$\operatorname{plot}\left(\mathrm{k}-1, \mathrm{abs}(\mathrm{yh}),{ }^{\prime} \mathrm{r}^{\prime}\right)$; hold on

$\operatorname{plot}\left(\mathrm{k}-1, \mathrm{abs}(\mathrm{yk}),{ }^{\prime} \mathrm{k}-{ }^{\prime}\right)$;

plot(k-1,abs(ya),'g-');

$\left.\operatorname{disp}\left(' \mathrm{fh}=\mathrm{fh} 0+\operatorname{real}(\mathrm{yh}(2)) * \cos \left(2 * \mathrm{pi}^{*} \mathrm{t} / \mathrm{N}\right)-\operatorname{imag}(\mathrm{yh}(2)) * \sin (2 * \mathrm{pi} * \mathrm{t} / \mathrm{N})\right)^{\prime}\right)$;

$\operatorname{disp}\left(' \mathrm{fh}=\mathrm{fh}+\mathrm{real}(\mathrm{yh}(3)) * \cos (2 * \mathrm{pi} * 2 * \mathrm{t} / \mathrm{N})-\operatorname{imag}(\mathrm{yh}(3)) * \sin (2 * \mathrm{pi} * 2 * \mathrm{t} / \mathrm{N}){ }^{\prime}\right) ;$

$\left.\operatorname{disp}\left({ }^{\prime} \mathrm{fh}=\mathrm{fh}+\mathrm{real}(\mathrm{yh}(4)) * \cos (2 * \mathrm{pi} * 3 * \mathrm{t} / \mathrm{N})-\operatorname{imag}(\mathrm{yh}(4)) * \sin \left(2 * \mathrm{pi}^{*} 3 * \mathrm{t} / \mathrm{N}\right)\right)^{\prime}\right)$

$\operatorname{disp}\left(' \mathrm{fh}=\mathrm{fh}+\mathrm{real}(\mathrm{yh}(5)) * \cos \left(2 * \mathrm{pi}^{*} 4 * \mathrm{t} / \mathrm{N}\right)-\operatorname{imag}(\mathrm{yh}(5)) * \sin (2 * \mathrm{pi} * 4 * \mathrm{t} / \mathrm{N}){ }^{\prime}\right)$;

$\operatorname{disp}\left(' \mathrm{fh}=\mathrm{fh}+\mathrm{real}(\mathrm{yh}(6)) * \cos \left(2 * \mathrm{pi}^{*} 5 * \mathrm{t} / \mathrm{N}\right)-\operatorname{imag}(\mathrm{yh}(6)) * \sin (2 * \mathrm{pi} * 5 * \mathrm{t} / \mathrm{N}){ }^{\prime}\right) ;$

$\left.\operatorname{disp}\left(' \mathrm{fh}=\mathrm{fh}+\mathrm{real}(\mathrm{yh}(7)) * \cos \left(2 * \mathrm{pi}^{*} 6 * \mathrm{t} / \mathrm{N}\right)-\operatorname{imag}(\mathrm{yh}(7))\right)^{*} \sin \left(2 * \mathrm{pi}{ }^{*} 6^{*} \mathrm{t} / \mathrm{N}\right) '\right)$;

fh0 


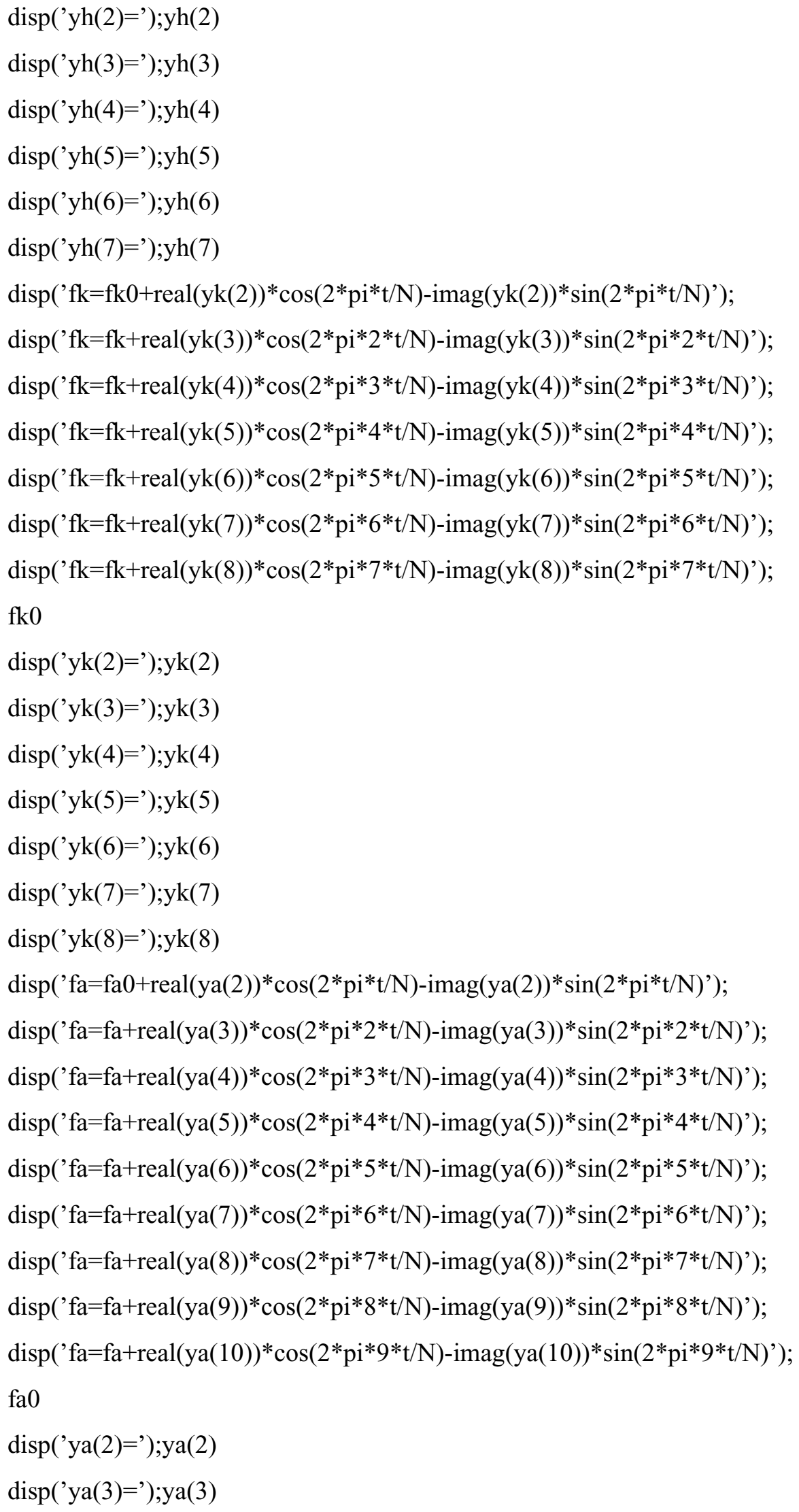




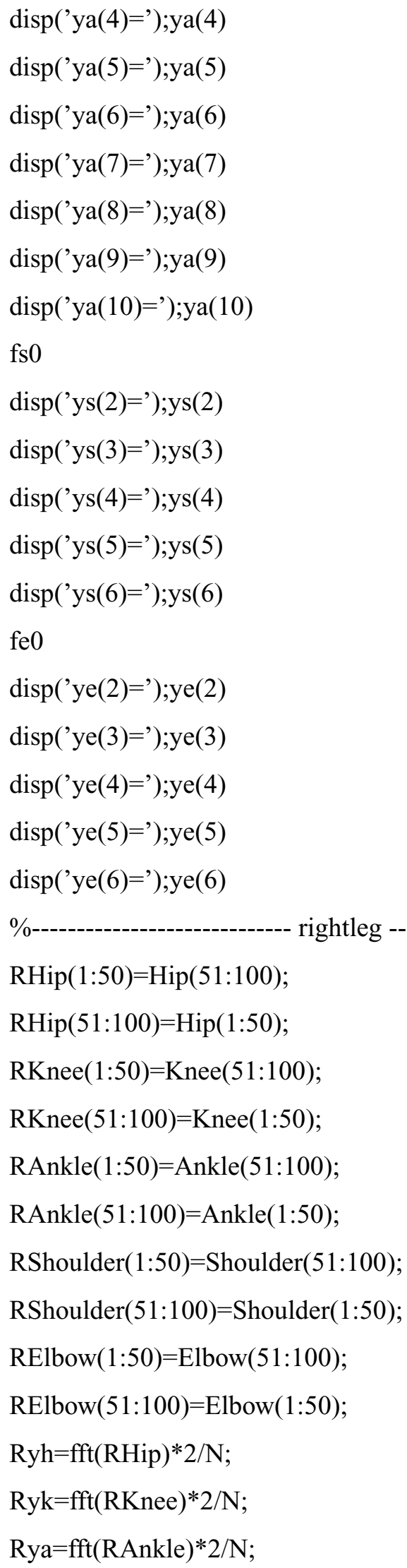


Rys $=\mathrm{fft}(\mathrm{RShoulder}) * 2 / \mathrm{N}$;

Rye $=\mathrm{fft}($ RElbow $) * 2 / \mathrm{N}$;

$\mathrm{Rfh} 0=\operatorname{Ryh}(1) / 2$;

$\mathrm{Rfh}=\mathrm{Rfh} 0+\operatorname{real}(\operatorname{Ryh}(2)) * \cos (2 * \mathrm{pi} * \mathrm{t} / \mathrm{N})-\operatorname{imag}(\operatorname{Ryh}(2)) * \sin (2 * \mathrm{pi} * \mathrm{t} / \mathrm{N})$;

$\mathrm{Rfh}=\mathrm{Rfh}+\operatorname{real}(\operatorname{Ryh}(3)) * \cos (2 * \mathrm{pi} * 2 * \mathrm{t} / \mathrm{N})-\operatorname{imag}(\operatorname{Ryh}(3)) * \sin (2 * \mathrm{pi} * 2 * \mathrm{t} / \mathrm{N})$;

$\mathrm{Rfh}=\mathrm{Rfh}+\operatorname{real}(\operatorname{Ryh}(4)) * \cos \left(2 * \mathrm{pi}^{*} 3 * \mathrm{t} / \mathrm{N}\right)-\operatorname{imag}(\operatorname{Ryh}(4)) * \sin \left(2 * \mathrm{pi}^{*} 3 * \mathrm{t} / \mathrm{N}\right)$;

$\mathrm{Rfh}=\mathrm{Rfh}+\operatorname{real}(\operatorname{Ryh}(5)) * \cos \left(2 * \mathrm{pi}^{*} 4 * \mathrm{t} / \mathrm{N}\right)-\operatorname{imag}(\operatorname{Ryh}(5)) * \sin \left(2 * \mathrm{pi}^{*} 4 * \mathrm{t} / \mathrm{N}\right)$;

$\mathrm{Rfh}=\mathrm{Rfh}+\operatorname{real}(\operatorname{Ryh}(6)) * \cos (2 * \mathrm{pi} * 5 * \mathrm{t} / \mathrm{N})-\mathrm{imag}(\operatorname{Ryh}(6)) * \sin (2 * \mathrm{pi} * 5 * \mathrm{t} / \mathrm{N})$;

$\mathrm{Rfh}=\mathrm{Rfh}+\operatorname{real}(\operatorname{Ryh}(7)) * \cos (2 * \mathrm{pi} * 6 * \mathrm{t} / \mathrm{N})-\mathrm{imag}(\operatorname{Ryh}(7)) * \sin (2 * \mathrm{pi} * 6 * \mathrm{t} / \mathrm{N})$;

$\operatorname{Rfk} 0=\operatorname{Ryk}(1) / 2$;

$\mathrm{Rfk}=\mathrm{Rfk} 0+\operatorname{real}(\operatorname{Ryk}(2)) * \cos (2 * \mathrm{pi} * \mathrm{t} / \mathrm{N})-\operatorname{imag}(\operatorname{Ryk}(2)) * \sin (2 * \mathrm{pi} * \mathrm{t} / \mathrm{N})$;

$\mathrm{Rfk}=\mathrm{Rfk}+\operatorname{real}(\operatorname{Ryk}(3)) * \cos \left(2 * \mathrm{pi}^{*} 2 * \mathrm{t} / \mathrm{N}\right)-\operatorname{imag}(\operatorname{Ryk}(3)) * \sin (2 * \mathrm{pi} * 2 * \mathrm{t} / \mathrm{N})$;

$\mathrm{Rfk}=\mathrm{Rfk}+\operatorname{real}(\operatorname{Ryk}(4)) * \cos \left(2 * \mathrm{pi}^{*} 3 * \mathrm{t} / \mathrm{N}\right)-\operatorname{imag}(\operatorname{Ryk}(4)) * \sin (2 * \mathrm{pi} * 3 * \mathrm{t} / \mathrm{N})$;

$\mathrm{Rfk}=\mathrm{Rfk}+\operatorname{real}(\operatorname{Ryk}(5)) * \cos \left(2 * \mathrm{pi}^{*} 4 * \mathrm{t} / \mathrm{N}\right)-\operatorname{imag}(\operatorname{Ryk}(5)) * \sin \left(2 * \mathrm{pi}^{*} 4 * \mathrm{t} / \mathrm{N}\right)$;

$\mathrm{Rfk}=\mathrm{Rfk}+\operatorname{real}(\operatorname{Ryk}(6)) * \cos (2 * \mathrm{pi} * 5 * \mathrm{t} / \mathrm{N})-\operatorname{imag}(\operatorname{Ryk}(6)) * \sin (2 * \mathrm{pi} * 5 * \mathrm{t} / \mathrm{N})$;

$\mathrm{Rfk}=\mathrm{Rfk}+\operatorname{real}(\operatorname{Ryk}(7)) * \cos (2 * \mathrm{pi} * 6 * \mathrm{t} / \mathrm{N})-\operatorname{imag}(\operatorname{Ryk}(7)) * \sin (2 * \mathrm{pi} * 6 * \mathrm{t} / \mathrm{N})$;

$\mathrm{Rfk}=\mathrm{Rfk}+\operatorname{real}(\operatorname{Ryk}(8)) * \cos \left(2 * \mathrm{pi}^{*} 7 * \mathrm{t} / \mathrm{N}\right)-\operatorname{imag}(\operatorname{Ryk}(8)) * \sin \left(2 * \mathrm{pi}^{*} 7 * \mathrm{t} / \mathrm{N}\right)$;

$\mathrm{Rfa} 0=\operatorname{Rya}(1) / 2$;

$\mathrm{Rfa}=\mathrm{Rfa} 0+\operatorname{real}(\mathrm{Rya}(2)) * \cos (2 * \mathrm{pi} * \mathrm{t} / \mathrm{N})-\operatorname{imag}(\mathrm{Rya}(2)) * \sin (2 * \mathrm{pi} * \mathrm{t} / \mathrm{N})$;

$\mathrm{Rfa}=\mathrm{Rfa}+\operatorname{real}(\operatorname{Rya}(3)) * \cos (2 * \mathrm{pi} * 2 * \mathrm{t} / \mathrm{N})-\operatorname{imag}(\operatorname{Rya}(3)) * \sin (2 * \mathrm{pi} * 2 * \mathrm{t} / \mathrm{N})$;

$\mathrm{Rfa}=\mathrm{Rfa}+\mathrm{real}(\mathrm{Rya}(4)) * \cos \left(2 * \mathrm{pi}^{*} 3 * \mathrm{t} / \mathrm{N}\right)-\operatorname{imag}(\operatorname{Rya}(4)) * \sin \left(2 * \mathrm{pi}^{*} 3 * \mathrm{t} / \mathrm{N}\right)$;

$\mathrm{Rfa}=\mathrm{Rfa}+\operatorname{real}(\mathrm{Rya}(5)) * \cos (2 * \mathrm{pi} * 4 * \mathrm{t} / \mathrm{N})-\operatorname{imag}(\operatorname{Rya}(5)) * \sin \left(2 * \mathrm{pi}^{*} 4 * \mathrm{t} / \mathrm{N}\right)$;

$\mathrm{Rfa}=\mathrm{Rfa}+\operatorname{real}(\mathrm{Rya}(6)) * \cos (2 * \mathrm{pi} * 5 * \mathrm{t} / \mathrm{N})-\operatorname{imag}(\mathrm{Rya}(6)) * \sin (2 * \mathrm{pi} * 5 * \mathrm{t} / \mathrm{N})$;

$\mathrm{Rfa}=\mathrm{Rfa}+\mathrm{real}(\operatorname{Rya}(7)) * \cos (2 * \mathrm{pi} * 6 * \mathrm{t} / \mathrm{N})-\operatorname{imag}(\operatorname{Rya}(7)) * \sin (2 * \mathrm{pi} * 6 * \mathrm{t} / \mathrm{N})$;

$\mathrm{Rfa}=\mathrm{Rfa}+\mathrm{real}(\mathrm{Rya}(8)) * \cos \left(2 * \mathrm{pi}^{*} 7 * \mathrm{t} / \mathrm{N}\right)-\operatorname{imag}(\operatorname{Rya}(8)) * \sin \left(2 * \mathrm{pi}^{*} 7 * \mathrm{t} / \mathrm{N}\right)$;

$\mathrm{Rfa}=\mathrm{Rfa}+\mathrm{real}(\mathrm{Rya}(9)) * \cos \left(2 * \mathrm{pi}^{*} 8 * \mathrm{t} / \mathrm{N}\right)-\operatorname{imag}(\operatorname{Rya}(9)) * \sin \left(2 * \mathrm{pi}^{*} 8 * \mathrm{t} / \mathrm{N}\right)$;

$\mathrm{Rfa}=\mathrm{Rfa}+\mathrm{real}(\operatorname{Rya}(10)) * \cos (2 * \mathrm{pi} * 9 * \mathrm{t} / \mathrm{N})-\operatorname{imag}(\operatorname{Rya}(10)) * \sin \left(2 * \mathrm{pi}^{*} 9 * \mathrm{t} / \mathrm{N}\right)$;

$\operatorname{Rfs} 0=\operatorname{Rys}(1) / 2$;

$\mathrm{Rfs}=\mathrm{Rfs} 0+\operatorname{real}(\operatorname{Rys}(2)) * \cos (2 * \mathrm{pi} * \mathrm{t} / \mathrm{N})-\operatorname{imag}(\operatorname{Rys}(2)) * \sin (2 * \mathrm{pi} * \mathrm{t} / \mathrm{N})$;

$\mathrm{Rfs}=\mathrm{Rfs}+\operatorname{real}(\operatorname{Rys}(3)) * \cos \left(2 * \mathrm{pi}^{*} 2 * \mathrm{t} / \mathrm{N}\right)-\operatorname{imag}(\operatorname{Rys}(3)) * \sin (2 * \mathrm{pi} * 2 * \mathrm{t} / \mathrm{N})$;

$\mathrm{Rfs}=\mathrm{Rfs}+\operatorname{real}(\operatorname{Rys}(4)) * \cos \left(2 * \mathrm{pi}^{*} 3 * \mathrm{t} / \mathrm{N}\right)-\operatorname{imag}(\operatorname{Rys}(4)) * \sin (2 * \mathrm{pi} * 3 * \mathrm{t} / \mathrm{N})$;

$\mathrm{Rfs}=\mathrm{Rfs}+\operatorname{real}(\operatorname{Rys}(5)) * \cos (2 * \mathrm{pi} * 4 * \mathrm{t} / \mathrm{N})-\operatorname{imag}(\operatorname{Rys}(5)) * \sin (2 * \mathrm{pi} * 4 * \mathrm{t} / \mathrm{N})$;

$\mathrm{Rfs}=\mathrm{Rfs}+\mathrm{real}(\operatorname{Rys}(6)) * \cos \left(2 * \mathrm{pi}^{*} 5 * \mathrm{t} / \mathrm{N}\right)-\operatorname{imag}(\operatorname{Rys}(6)) * \sin \left(2 * \mathrm{pi}^{*} 5 * \mathrm{t} / \mathrm{N}\right)$; 







$$
\begin{aligned}
& \operatorname{disp}\left({ }^{\prime} \operatorname{Ryk}(2)='\right) ; \operatorname{Ryk}(2) \\
& \operatorname{disp}(\operatorname{Ryk}(3)=') ; \operatorname{Ryk}(3) \\
& \operatorname{disp}(' \operatorname{Ryk}(4)=\text { '); } \operatorname{Ryk}(4) \\
& \operatorname{disp}(' \operatorname{Ryk}(5)=') ; \operatorname{Ryk}(5) \\
& \operatorname{disp}\left({ }^{\prime} \operatorname{Ryk}(6)='\right) ; \operatorname{Ryk}(6) \\
& \operatorname{disp}(' \operatorname{Ryk}(7)=') ; \operatorname{Ryk}(7) \\
& \operatorname{disp}\left({ }^{\prime} \operatorname{Ryk}(8)='\right) ; \operatorname{Ryk}(8) \\
& \text { Rfa0 } \\
& \operatorname{disp}(' \operatorname{Rya}(2)=') ; \operatorname{Rya}(2) \\
& \operatorname{disp}(' \operatorname{Rya}(3)=') ; \operatorname{Rya}(3) \\
& \operatorname{disp}(' \operatorname{Rya}(4)=') ; \operatorname{Rya}(4) \\
& \operatorname{disp}(' \operatorname{Rya}(5)=') ; \operatorname{Rya}(5) \\
& \operatorname{disp}(' \operatorname{Rya}(6)=\text { '); } \operatorname{Rya}(6) \\
& \operatorname{disp}(' \operatorname{Rya}(7)=') ; \operatorname{Rya}(7) \\
& \operatorname{disp}(' \operatorname{Rya}(8)=') ; \operatorname{Rya}(8) \\
& \operatorname{disp}(' \operatorname{Rya}(9)=') ; \operatorname{Rya}(9) \\
& \operatorname{disp}(' \operatorname{Rya}(10)=\text { ');Rya(10) } \\
& \text { Rfs0 } \\
& \operatorname{disp}(\text { 'Rys(2)='); } \operatorname{Rys}(2) \\
& \operatorname{disp}(' \operatorname{Rys}(3)=') ; \operatorname{Rys}(3) \\
& \operatorname{disp}(' \operatorname{Rys}(4)=\text { )'); Rys(4) } \\
& \operatorname{disp}(' \operatorname{Rys}(5)=') ; \operatorname{Rys}(5) \\
& \operatorname{disp}(' \operatorname{Rys}(6)=') \text {;Rys(6) } \\
& \text { Rfe0 } \\
& \operatorname{disp}(' \operatorname{Rye}(2)=\text { )'); Rye(2) } \\
& \operatorname{disp}(' \operatorname{Rye}(3)=') ; \operatorname{Rye}(3) \\
& \operatorname{disp}(\text { 'Rye(4)='); Rye(4) } \\
& \operatorname{disp}(' \operatorname{Rye}(5)=\text { )'); Rye(5) } \\
& \text { disp('Rye(6)=');Rye(6) }
\end{aligned}
$$

\section{B.4 hipcam.m}

This code is compiled to calculate the cam profile \%-------- Cam profile for hip joint ------------ 







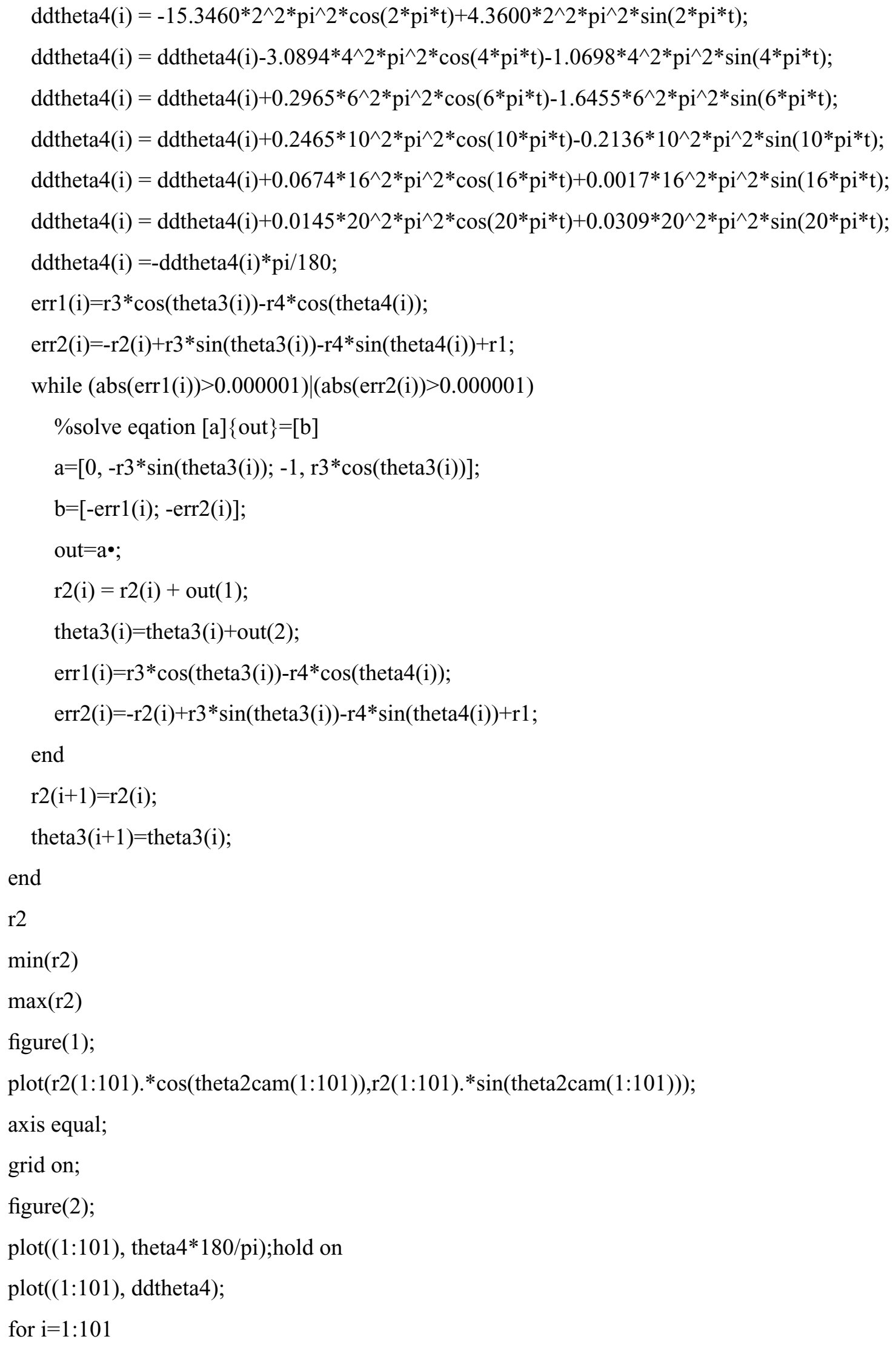


$\mathrm{t}=(\mathrm{i}-1) / 100$

theta 2 cam $(\mathrm{i})=$ theta $2+2 * \mathrm{pi} * \mathrm{t}$;

$\%$---- theta4 is from Fourier series used in simulation ----

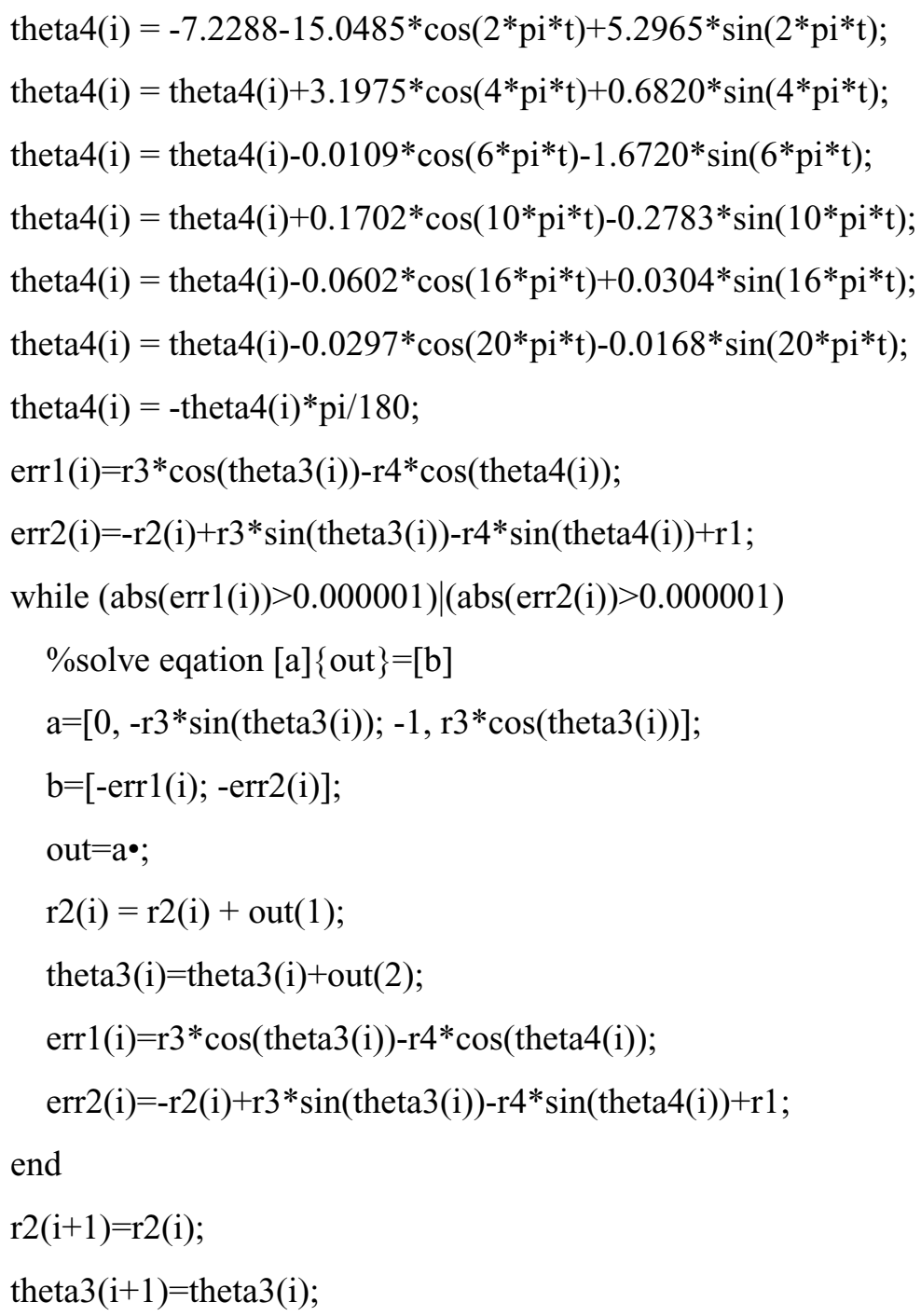




\section{B.5 Data_processing.m}

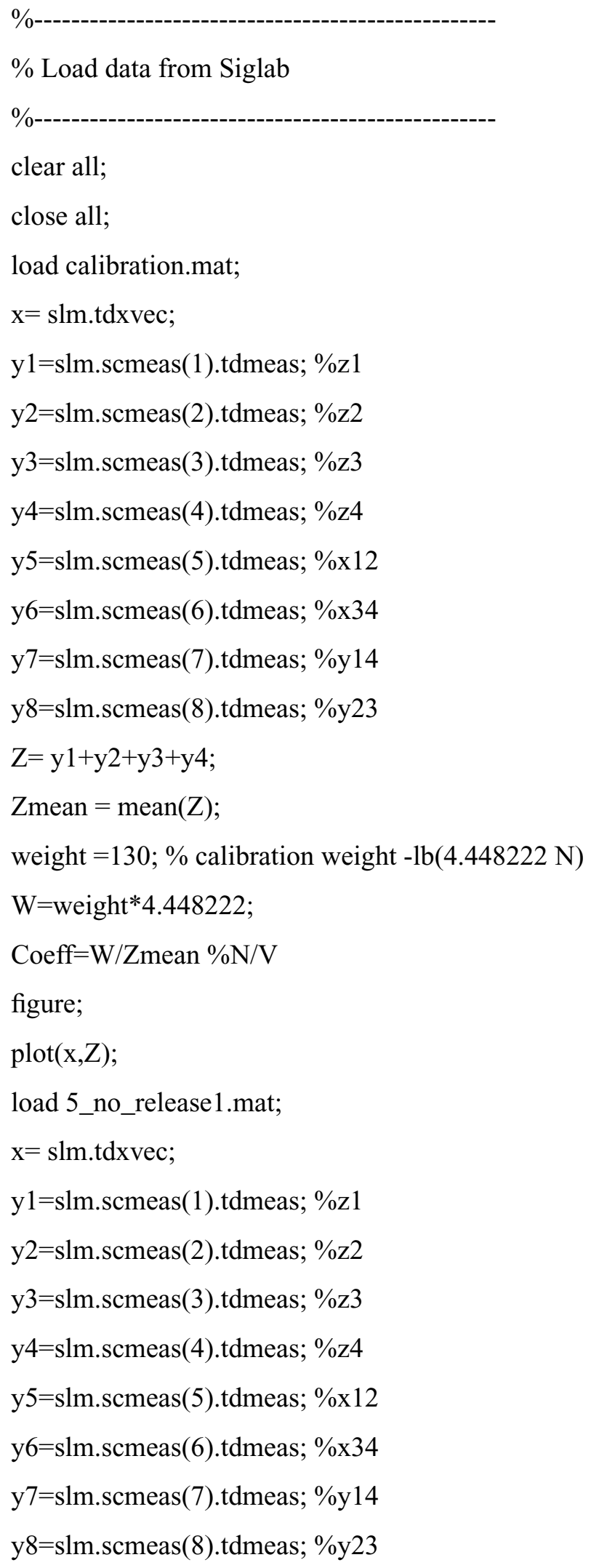


$\mathrm{Z}=\mathrm{y} 1+\mathrm{y} 2+\mathrm{y} 3+\mathrm{y} 4$

$\mathrm{X}=\mathrm{y} 5+\mathrm{y} 6$;

$\mathrm{Y}=\mathrm{y} 7+\mathrm{y} 8$

Impact $1=Z *$ Coeff;

Friction $1=\operatorname{sqrt}\left(\mathrm{X} .{ }^{\wedge} 2+\mathrm{Y} .{ }^{\wedge} 2\right)^{*}$ Coeff;

load 5_with_release1.mat;

$\mathrm{x}=\mathrm{slm} \cdot \mathrm{tdxvec}$;

y1=slm.scmeas(1).tdmeas; \%z1

y2 $=$ slm.scmeas(2).tdmeas; \%z2

$\mathrm{y} 3=$ slm.scmeas(3).tdmeas; \%z3

$\mathrm{y} 4=$ slm.scmeas(4).tdmeas; \%z4

y5 $=$ slm.scmeas(5).tdmeas; \%x12

y6=slm.scmeas(6).tdmeas; \%x34

$\mathrm{y} 7=$ slm.scmeas(7).tdmeas; \%y14

y8 $=$ slm.scmeas(8).tdmeas; \%y23

$\mathrm{Z}=\mathrm{y} 1+\mathrm{y} 2+\mathrm{y} 3+\mathrm{y} 4$

$\mathrm{X}=\mathrm{y} 5+\mathrm{y} 6$

$\mathrm{Y}=\mathrm{y} 7+\mathrm{y} 8$

Impact $2=Z *$ Coeff;

Friction $2=\operatorname{sqrt}\left(X .^{\wedge} 2+Y^{\wedge}{ }^{\wedge}\right) * \operatorname{Coeff} ;$

figure;

subplot(2,1,1); plot(x, Impact1); hold on

$\operatorname{plot}(\mathrm{x}$, Impact2, 'r');

title('Impact force');

xlabel('time (S)'); ylabel('Force (N)');

legend('Without release movement', 'With release movement');

grid on;

subplot(2,1,2); plot(x, Friction1);hold on

plot(x, Friction2, 'r');

title('Friction');

xlabel('time (S)'); ylabel('Force (N)');

legend('Without release movement', 'With release movement');

grid on; 